\title{
Review of searches for rare processes and physics beyond the Standard Model at HERA
}

\author{
David M. South ${ }^{1, \mathrm{a}}$, Monica Turcato ${ }^{2,3, \mathrm{~b}}$ \\ ${ }^{1}$ Deutsches Elektronen Synchrotron, Hamburg, Germany \\ ${ }^{2}$ Institute of Experimental Physics, Hamburg University, Hamburg, Germany \\ ${ }^{3}$ Present address European X-ray Free-Electron Laser Facility GmbH, Hamburg, Germany
}

Received: 12 May 2016 / Accepted: 18 May 2016 / Published online: 18 June 2016

(C) The Author(s) 2016. This article is published with open access at Springerlink.com

\begin{abstract}
The electron-proton collisions collected by the $\mathrm{H} 1$ and ZEUS experiments at HERA comprise a unique particle physics data set, and a comprehensive range of measurements has been performed to provide new insight into the structure of the proton. The high centre of mass energy at HERA has also allowed rare processes to be studied, including the production of $W$ and $Z^{0}$ bosons and events with multiple leptons in the final state. The data have also opened up a new domain to searches for physics beyond the Standard Model including contact interactions, leptoquarks, excited fermions and a number of supersymmetric models. This review presents a summary of such results, where the analyses reported correspond to an integrated luminosity of up to $1 \mathrm{fb}^{-1}$, representing the complete data set recorded by the $\mathrm{H} 1$ and ZEUS experiments.
\end{abstract}

\section{Introduction}

The Standard Model (SM) of particle physics [1-3], which describes the fundamental building blocks of nature and their interactions, is one of the success stories of the last 50 years in science. The theoretical development and subsequent experimental confirmation of the SM, which describes the elementary particles and their weak, electromagnetic and strong interactions, has been made possible by a variety of particle accelerators and their associated experimental programmes operated during this time. The successful exploration of the electroweak sector, from the discovery of weak neutral currents in the bubble chamber experiments of the early 1970s to the subsequent observation of the weak bosons at the SPS at CERN in the early 1980s, greatly influenced the direction of research and detector design, as well as the type and energy

\footnotetext{
a e-mail: david.south@ desy.de

be-mail: monica.turcato@xfel.eu
}

of machines developed in the following decades. Different types of programmes have been formed more recently, based on making precision measurements such as the LEP experiments at CERN, BaBar at SLAC and Belle at KEK, to all out discovery machines such as the Tevatron at Fermilab or the LHC at CERN. In the last few years, the LHC has produced one of the major physics results of this exciting half century of particle physics, with the observation $[4,5]$ of a new narrow resonance consistent with the long sought Higgs boson [6-8].

The HERA accelerator at DESY is a unique achievement, in that it is the world's only electron ${ }^{1}$-proton collider to be constructed, thus providing an unrivalled physics programme to the high-energy physics community. Bringing into collision point-like leptons with finite sized hadrons, HERA may be thought of as a powerful electron microscope, a tool to make precise measurements of the structure of the proton and to investigate the nature of strong force binding it together. The experimental data from the $e p$ collisions at HERA also allow rare processes to be studied, typically those involving the electroweak gauge bosons, and to search for physics beyond the Standard Model (BSM), in analyses complementary to those at other colliders and sometimes unique to $e p$ physics.

This review presents a summary of the measurements of rare processes and BSM searches based on experimental data taken by the H1 and ZEUS experiments at HERA. The majority of the presented analyses utilise the complete data sets of the experiments, which in combination amounts to an integrated luminosity of $1 \mathrm{fb}^{-1}$. A brief introduction to the kinematics of $e p$ scattering is followed by a description of HERA and the DESY accelerator facility and the H1 and ZEUS experiments. An outline of particle identification,

\footnotetext{
1 The term "electron" is used generically to refer to both electrons and positrons, unless otherwise stated.
} 
event reconstruction and simulation is then given, describing the key methods employed in the data analysis performed by each experiment.

To effectively perform searches for rare processes and new physics in the data, it is important to have a good understanding and level of confidence in the description of SM physics. Therefore, a summary of the main experimental results on deep inelastic scattering (DIS) at large momentum transfer is given in Sect. 8, which are performed in a similar kinematic region to the majority of the results presented in this review. This is particularly important for the first category of searches, where deviations from SM expectation of DIS events may reveal new physics, namely searches for contact interactions and leptoquarks as described in Sect. 9. Dedicated searches for first, second and third generation leptoquarks are then presented in Sect. 10.

Rare processes with high transverse momentum leptons in the final state are investigated in Sects. 11 and 12, featuring measurements of lepton pair and $W$ production. Potential new signals are also investigated in the context of these analyses in Sects. 11.3 and 13. The production of $Z^{0}$ bosons is examined by the ZEUS experiment using the hadronic decay channel in Sect. 14. A model-independent "general" search for new physics is performed by $\mathrm{H} 1$ as described in Sect. 15, where all final states are examined containing high transverse momentum entities. Searches for new physics in the context of specific models are then presented, for excited fermions and supersymmetry in Sects. 16 and 17, respectively. Finally a novel search for magnetic monopoles performed by the $\mathrm{H} 1$ experiment is presented in Sect. 18, before a summary of the presented results is given together with an outlook in Sect. 19.

\section{The kinematics of electron-proton scattering}

Figure 1 shows a diagram of the interaction $e p \rightarrow \ell X$, where a virtual photon $(\gamma)$, or heavy vector boson $\left(Z^{0}\right.$ or $\left.W\right)$ is exchanged between the incoming electron $(e)$ and proton $(p)$. As the mediator boson between the electron and the proton can be either a photon or a heavy vector boson, due to the

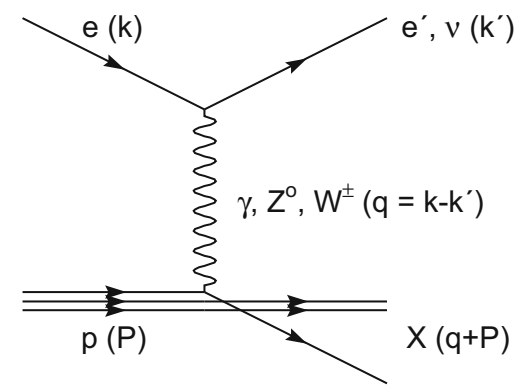

Fig. 1 The scattering of electrons and protons at HERA. The fourmomenta of the particles are indicated in the parentheses. The exchanged gauge boson is a photon $(\gamma)$ or $Z^{0}$ boson in NC interactions and a $W$ boson in CC interactions high centre of mass energy at HERA, QED and weak interactions may be studied simultaneously, testing the electroweak theory. The outgoing particles are made up of the scattered lepton $(\ell)$ and those contained in the hadronic final state $(X)$. In the case of neutral current $(\mathrm{NC})$ interactions, the exchange is mediated by a $\gamma$ or $Z^{0}$, so that an electron $\left(e^{\prime}\right)$ is present in the final state. In charged current (CC) interactions, the weak exchange of the $W$ boson results in a final-state neutrino (v).

The four-momenta of the initial-state electron and proton are denoted $k$ and $P$, so that the centre of mass energy $\sqrt{s}$ is given by

$s=(k+P)^{2}$.

Neglecting the masses of the incoming particles, this can be approximated by $\sqrt{s} \approx 4 E_{e}^{0} E_{p}^{0}$, where $E_{e}^{0}\left(E_{p}^{0}\right)$ is the energy of the initial-state electron (proton). The square of the fourmomentum transfer (which is equal to the mass squared of the virtual boson), $q^{2}<0$, determines the hardness, or in other words, the resolving power of the interaction. The negative four-momentum transfer squared is defined as

$Q^{2}=-q^{2}=-\left(k-k^{\prime}\right)^{2}$

and the invariant mass $W$ of the hadronic final-state $X$ is given by

$W^{2}=(q+P)^{2}$.

Interactions at HERA are denoted elastic if the proton remains intact, quasi-elastic if the proton dissociates into a low-mass hadronic system, or inelastic if the proton breaks up. Deep inelastic scattering (DIS) events are characterised as having large momentum transfer $Q^{2} \gg m_{p}^{2}$ and are highly inelastic, $W \gg m_{p}$, where $m_{p}$ is the mass of the proton.

The fraction of the proton momentum carried by the struck parton is given by the quantity Bjorken $x$, where

$x=\frac{Q^{2}}{2 P \cdot q}$

under the assumption of massless quarks. The inelasticity of the interaction, $y$, is given by

$y=\frac{q \cdot P}{k \cdot P}$

and is equal to the fraction of the incident electron momenta carried by the exchange boson in the rest frame of the proton. Both $x$ and $y$ take values between 0 and 1 since they describe fractions of momenta, as defined above. The above quantities are related by

$Q^{2} \approx s x y$, 


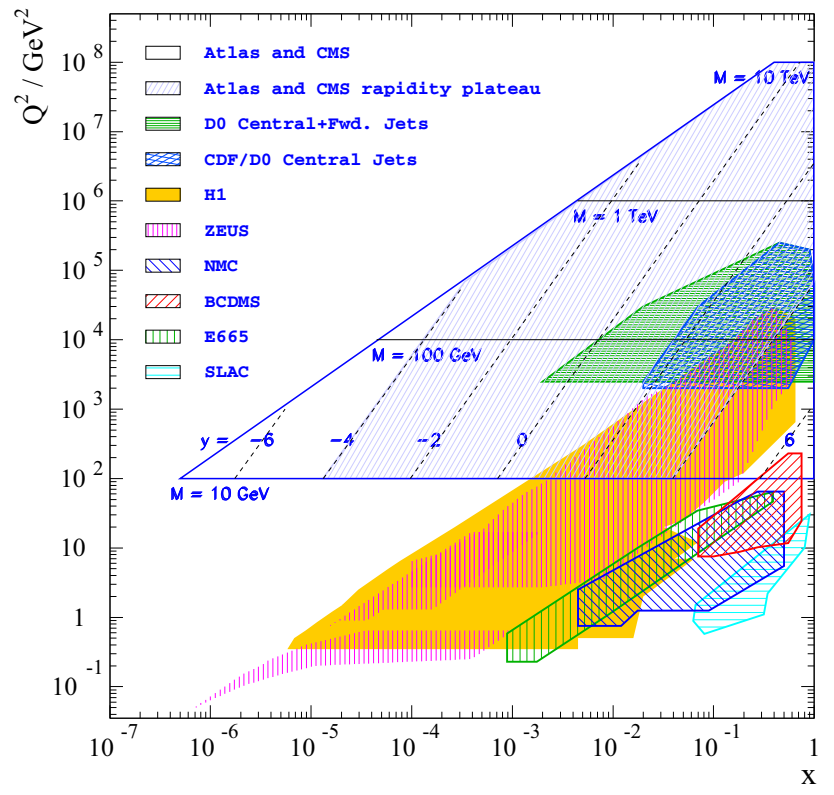

Fig. 2 Regions of phase space in the $x-Q^{2}$ kinematic plane covered by several collider and fixed-target experiments

resulting in a maximum squared four-momentum exchange equal to the centre of mass energy squared $s$.

The value of $Q^{2}$ is a measure of the virtuality of the exchange boson: for $Q^{2} \approx 0$, the photon is considered to be almost real, and in such interactions the initial-state electron scatters under very small angles. An interaction at higher $Q^{2}$ represents a more energetic exchange, resulting in a higher resolution of the parton participating in the interaction: one of the three valence quarks, a sea quark or a gluon. Hence the exchanged boson acts as a probe for the determination of the structure of the proton and depending on the scattering angle and energy of the outgoing lepton, different $Q^{2}$ and $x$ ranges can be investigated, thus examining the electromagnetic and weak charge distribution inside the proton.

The advent of the HERA collider in 1992 made it possible to explore a much wider region in $x$ and $Q^{2}$ than that previously accessible at fixed-target experiments, with measurements possible down to $10^{-5}$ in $x$ and low $Q^{2}$, in particular in the non-perturbative region and up to a $Q^{2}$ of $10^{4} \mathrm{GeV}^{2}$ in the valence (high- $x$ ) region. The kinematic coverage of the HERA experiments in the $x-Q^{2}$ plane is shown in Fig. 2, compared to that of several fixed-target DIS experiments as well as the phase space covered by the hadron-hadron collision experiments at both the Tevatron $(p \bar{p})$ and the LHC $(p p)$. By exploiting QCD factorisation [9] and utilising the DGLAP [10-12] parton evolution scheme, the HERA parton distribution functions (PDFs) derived from $\mathrm{H} 1$ and ZEUS measurements across a large range in $x$ can be used as input to calculate predictions for the $\mathrm{LHC}$ at much higher values of $Q^{2}$. We will return to these measurements and the calculation and impact of the HERA PDFs in Sect. 8.

\section{The HERA collider at DESY}

Hadron Electron Ring Anlage (HERA) [13] is so far the only lepton-proton collider in the world to have been constructed. ${ }^{2}$ It was located at the Deutsches Electronen Synchrotron (DESY) laboratory, as pictured in the upper half of Fig. 3, and was in operation during the years 1992 to 2007. The HERA machine accelerated and brought into collision $27.6 \mathrm{GeV}$ electrons or positrons with $920 \mathrm{GeV}$ protons, ${ }^{3}$ resulting in a centre of mass energy of $319 \mathrm{GeV}$. At the time, this energy represented more than an order of magnitude increase with respect to the previous fixed-target experiments and consequently a new and wider kinematic region was accessible for the first time at HERA. In the final data taking period the proton beam was accelerated to lower energies, first $460 \mathrm{GeV}$ and then $575 \mathrm{GeV}$, in order to provide data used for a direct measurement of the longitudinal structure function $F_{L}$ (see Sect. 8).

The $6.3 \mathrm{~km}$ long HERA tunnel is located between 15 and 30 metres below ground, in which electrons and protons were accelerated in two counter-rotating rings. Four experiments were located in experimental halls around the HERA ring: Electron-proton collisions occurred at two interaction points, one in the North Hall where the H1 experiment $[15,16]$ was located, the other in the South Hall where the ZEUS experiment [17] could be found. The HERMES experiment [18] in the East Hall studied the spin structure of the nucleon using collisions of the lepton beam on an internal polarised gas target. The HERA-B experiment $[19,20]$ in the West Hall was built to use collisions of the proton beam halo with a wire target in order to produce $B$-mesons for the study of $C P$ violation in the $B-\bar{B}$ system. The layout of the HERA ring and the system of pre-accelerators at DESY is illustrated in the lower half of Fig. 3.

The proton beam began as negative hydrogen ions $\left(\mathrm{H}^{-}\right)$ accelerated in a linear accelerator to $50 \mathrm{MeV}$. The electrons were then stripped off the $\mathrm{H}^{-}$ions to obtain protons, which were injected into the proton synchrotron DESY III, accelerated up to $7.5 \mathrm{GeV}$, and transferred to the PETRA ring, where they were accelerated to $40 \mathrm{GeV}$. The protons were then finally injected in three shots into the HERA proton storage ring, which is made up of superconducting magnets with a maximum field of $4.65 \mathrm{~T}$, where they were then accelerated to the nominal beam energy of $920 \mathrm{GeV}$.

The electron (positron) pre-acceleration chain began in a linear accelerator, LINAC I (LINAC II), where the lep-

\footnotetext{
2 The conceptual design report of the proposed $\mathrm{LHeC}$ project, a machine to collide a high-energy electron beam with the hadron beams of the LHC, is now available [14].

3 The proton-beam energy was $820 \mathrm{GeV}$ from 1992 to 1997 , resulting in a centre of mass energy of $300 \mathrm{GeV}$. The data recorded during this period amounts to less than $10 \%$ of the total integrated luminosity yield.
} 

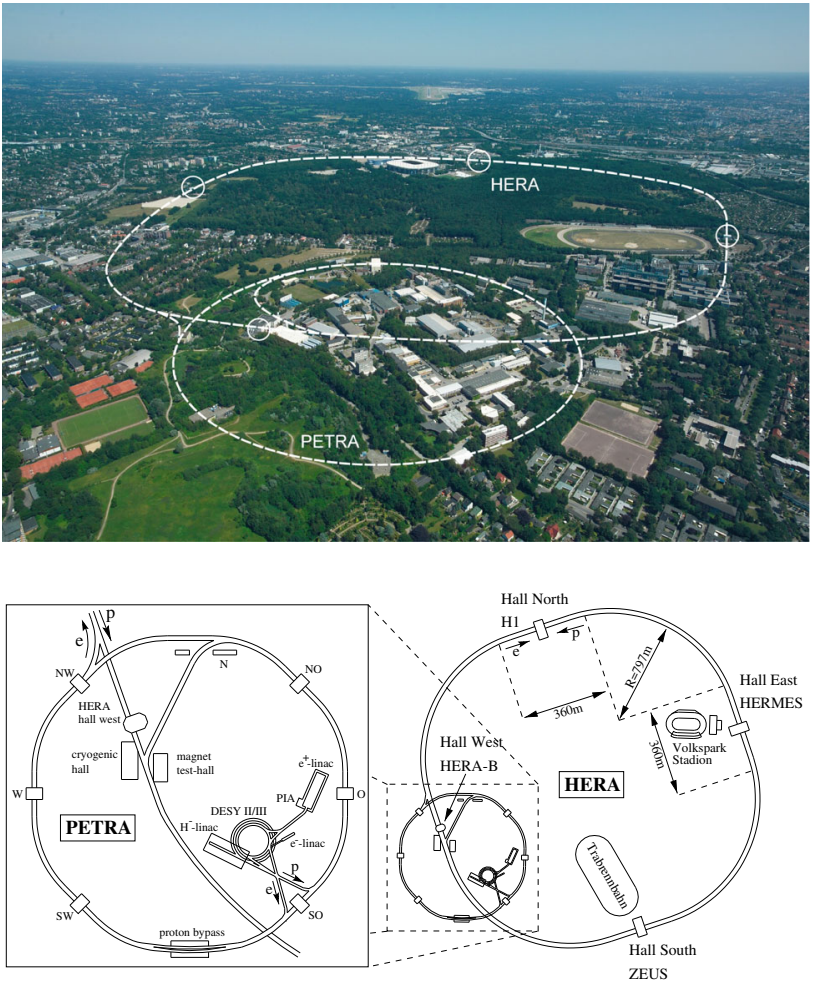

Fig. 3 The DESY research centre in Hamburg, Germany. In the upper photograph, the dashed circles show the path of the underground ring accelerators PETRA and HERA; the four large halls containing the HERA detectors are indicated by the smaller, solid circles. The lower diagram details the system of pre-accelerators employed in order to produce the proton and electron beams that were brought into collision at the $\mathrm{H} 1$ and ZEUS experiments

tons were accelerated up to $450 \mathrm{MeV}$. The leptons were then injected into the electron synchrotron DESY II, accelerated to $7 \mathrm{GeV}$ and, similarly to the protons, transferred to the PETRA ring, where they reached an energy of $14 \mathrm{GeV}$. Injection transfer into the HERA ring followed, where they were accelerated to the nominal lepton-beam energy of $27.6 \mathrm{GeV}$ using conventional magnets with a maximum field of $0.165 \mathrm{~T}$.

Up to 210 bunches of leptons and protons were accelerated in the HERA ring, spaced at $96 \mathrm{~ns}$ intervals. Only 175 bunches were typically used for collisions, where the remainder were used as pilot bunches to study background rates arising from interactions of the beams with residual gas in the beam pipe. When the proton bunches were compressed by HERA during acceleration, small secondary or satellite bunches were formed, separated from the main bunch by up to $8 \mathrm{~ns}$.

The data taking at HERA may be divided into two distinct periods: HERA I, which was from 1994 until 2000, and HERA II, from 2003 until 2007. A luminosity upgrade [21] of the machine took place between the two data taking periods and brought an observed increase in the luminosity delivered to the experiments from $1.5 \times 10^{31} \mathrm{~cm}^{-2} \mathrm{~s}^{-1}$ in the HERA I phase up to a peak value of $5.0 \times 10^{31} \mathrm{~cm}^{-2} \mathrm{~s}^{-1}$,

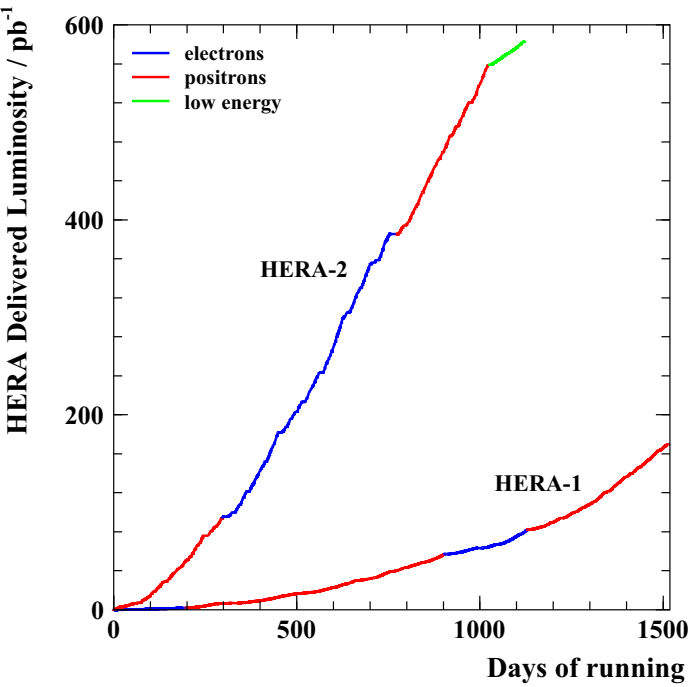

Fig. 4 A summary of the integrated luminosity delivered by the HERA collider during the HERA I (1992-2000) and HERA II (2003-2007) phases. The different electron and positron running periods are indicated, as well as the data taken at lower proton-beam energies in 2007

achieved during HERA II $e^{-} p$ running. The integrated luminosity delivered by the HERA accelerator is shown in Fig. 4.

The integrated luminosity collected for analysis by $\mathrm{H} 1$ and ZEUS amounts to about $0.5 \mathrm{fb}^{-1}$ per experiment. This is less than the delivered integrated luminosity, as quality conditions are applied to the data used for analysis, such as requirements on the high voltage status of the various detector subsystems (see Sect. 4). The luminosity is measured by both experiments from the rate of the well understood QED Bethe-Heitler process $e p \rightarrow e p \gamma$. As the photon is emitted almost collinear to the incoming electron, it is detected using devices located close to the beam line beyond the main detectors. A photon detector [22-24] is employed by $\mathrm{H} 1$ and the ZEUS experiment uses two independent systems, a photon calorimeter [25-27] and a magnetic spectrometer [28]. A recent analysis [29] of Compton scattering events provided an alternative and improved measurement of the luminosity recorded by the $\mathrm{H} 1$ experiment. The integrated luminosities of the data sets ${ }^{4}$ are detailed in Table 1.

Another feature of the HERA II upgrade was the use of a longitudinally polarised lepton beam. As the lepton beam circulated in HERA it naturally became transversely polarised via the Sokolov-Ternov effect $[30,31]$. The typical polarisation build-up time for the HERA accelerator was approximately $40 \mathrm{~min}$. At HERA II, spin rotators installed on either side of the $\mathrm{H} 1$ and ZEUS detectors changed the transverse polarisation of the beam into longitudinal polar-

\footnotetext{
${ }^{4}$ Note that for some analyses presented in this review the integrated luminosity may vary from this table. For example, some searches do not require a good polarisation measurement and this results in a higher luminosity yield from HERA II. In such cases, the integrated luminosity of the analysed data sets is given in the text.
} 
Table 1 Integrated luminosity $\mathcal{L}$ and luminosty-weighted average lepton-beam polarisation $\mathcal{P}_{e}$ of the $\mathrm{H} 1$ and ZEUS data. The centre of mass energy $\sqrt{s}$ of the data is $319 \mathrm{GeV}$, except for the 1994-1997 data, which is $301 \mathrm{GeV}$

\begin{tabular}{llllll}
\hline Data set & & H1 & \multicolumn{2}{l}{ ZEUS } & \\
\hline & & $\mathcal{L}\left(\mathrm{pb}^{-1}\right)$ & $\mathcal{P}_{e}(\%)$ & $\mathcal{L}\left(\mathrm{pb}^{-1}\right)$ & $\mathcal{P}_{e}(\%)$ \\
\hline 1994-1997 & $e^{+} p$ & 36 & 0 & 48 & 0 \\
$1998-1999$ & $e^{-} p$ & 16 & 0 & 16 & 0 \\
1999-2000 & $e^{+} p$ & 65 & 0 & 63 & 0 \\
HERA II & $e^{+} p$ & 98 & +32 & 91 & +32 \\
& & 82 & -38 & 68 & -37 \\
HERA II & $e^{-} p$ & 46 & +37 & 71 & +29 \\
& & 103 & -26 & 99 & -27 \\
\hline
\end{tabular}

isation and back again. The lepton-beam polarisation was measured using two independent polarimeters, the transverse polarimeter (TPOL) [32] and the longitudinal polarimeter (LPOL) [33]. Both devices exploited the spin-dependent cross section for Compton scattering of circularly polarised photons off positrons to measure the lepton-beam polarisation [34].

During the HERA II period, the machine was run in both left-handed and right-handed polarisation modes, where the average beam polarisation is given by

$\mathcal{P}_{e}=\left(N_{R}-N_{L}\right) /\left(N_{R}+N_{L}\right)$

and $N_{R}\left(N_{L}\right)$ is the number of right- (left-)handed leptons in the beam. Accordingly, four distinct data sets were recorded by the experiments at HERA II, by running with either left- or right-handed polarised electrons or positrons. The luminosity weighted average polarisations of the HERA II data sets are also given in Table 1.

\section{Particle physics detectors}

A general purpose particle physics experiment is normally composed of a series of different detectors surrounding the interaction region, which is the nominal point where the two counter-rotating beams of particles are brought into collision. Each detector identifies and measures particles produced in the interaction by taking advantage of their different properties. Figure 5 shows a general layout of a particle physics experiment: a tracking device in a magnetic field is surrounded by an electromagnetic and a hadronic calorimeter, and finally by muon detectors. Particles produced in the interaction region traverse these detectors sequentially from the collision point outwards, leaving different signatures depending on their physics properties.

The first detector surrounding the interaction region is a tracking system, which records the passage of charged particles. This system may be comprised of multiple detectors, such as drift chambers and silicon trackers. Charged particles leave their energy in these detectors via ionisation, and due to

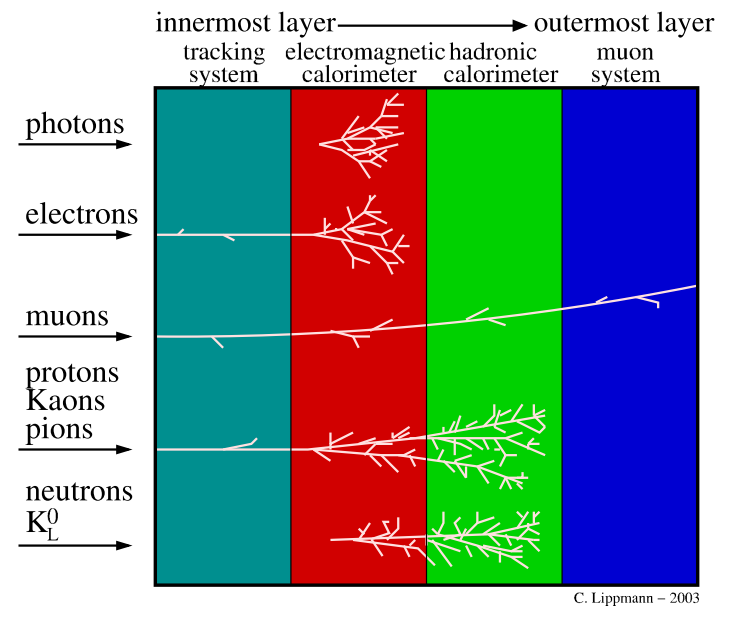

Fig. 5 Typical components of a particle physics detector. Different types of particle leave different signatures in the detector, allowing particle identification to be performed [35]

the low material budget only a small fraction of the particle energy is lost in such devices. In general neutral particles are not seen as tracks, but photons may convert into an electronpositron pair which can then be detected in the tracker. Tracks are reconstructed by detecting the particle energy lost along their path inside the detector. A magnetic field, which is parallel to the beam direction, allows the particle momentum and charge to be determined, based on the curvature of the tracks. The characteristic pattern of energy loss in the tracker may also be used to perform particle identification, allowing the separation of electrons, pions, kaons and protons at low momenta.

Moving outwards from the interaction region, the tracking system is enclosed inside a calorimeter. Calorimeters measure both neutral and charged particles by completely degrading them and absorbing all of the energy they deposit. A typical design is a sampling calorimeter, in which layers of active material such as scintillator or liquified noble gas are interleaved by layers of absorber such lead or depleted uranium. A calorimeter is also able to determine if a particle has electromagnetic or hadronic interaction by examining the pattern of the energy loss. This allows the separation of electron and photons from other particles which undergo 
hadronic interactions. A calorimeter is designed such that electrons and photons leave all of their energy in the electromagnetic section, which is closer to the interaction region and typically many radiation lengths deep. The electromagnetic interactions occur rapidly with the nuclei in the absorbing layers via the bremsstrahlung and pair-production processes. As electrons and photons cannot be distinguished using the calorimeter alone, this is done by spatially matching the electromagnetic energy deposit to a track in the tracking system. Hadrons penetrate more deeply in the detector, leaving energy deposits not only in the electromagnetic calorimeter but also in the hadronic section, where they interact strongly with the nuclei of the absorbing layers, elastically and inelastically, resulting in a shower composed of secondary hadrons. The characteristic length of the hadronic shower, the interaction length, is much longer than the radiation length for the same material and the shower, which also contains an electromagnetic component, is typically much broader than a purely electromagnetic interaction, allowing the two shower types to be distinguished. A full discussion of the separation of electromagnetic and hadronic showers can be found elsewhere [36].

Muons do not interact like hadrons via the strong force and do not radiate via bremsstrahlung as much as electrons due to their heavier mass, losing their energy only via ionisation and behaving like a minimum ionising particle. They therefore penetrate beyond the main calorimeters and additional, dedicated muon detectors are installed as the outermost layer of the detector. In order to make a muon momentum measurement, information from these outermost detectors is typically matched to a track measured in the tracking system.

A detailed description of the $\mathrm{H} 1$ and ZEUS detectors can be found elsewhere [15-17]. Both detectors are described by a right-handed cartesian coordinate system $(x, y, z)$ with the nominal interaction point defined at the origin, $+x$ pointing towards the centre of the ring, $+y$ pointing vertically upwards and $+z$ in the direction of the incoming proton beam (also referred to as the forward direction). The corresponding spherical coordinate system $(r, \theta, \phi)$ is defined so that $\theta=0^{\circ}$ is in the proton direction and consequently $\theta=180^{\circ}$ is in the electron (backward) direction.

The $\mathrm{H} 1$ and ZEUS detectors are illustrated in Fig. 6. Both designs feature tracking detectors closest to the beam pipe, which runs through the centre, surrounded by electromagnetic and hadronic calorimetry, which is enclosed in a muon system. Two striking features can be seen in the design of both the H1 and ZEUS detectors. Firstly, almost complete coverage is achieved around the interaction point. This not only allows particles produced in the $e p$ interaction to be almost completely contained, but also results in a reliable calculation of any net transverse momentum imbalance in an event, which is important if the resulting final state includes neutrinos. Secondly, both designs are very asymmetric, cor-
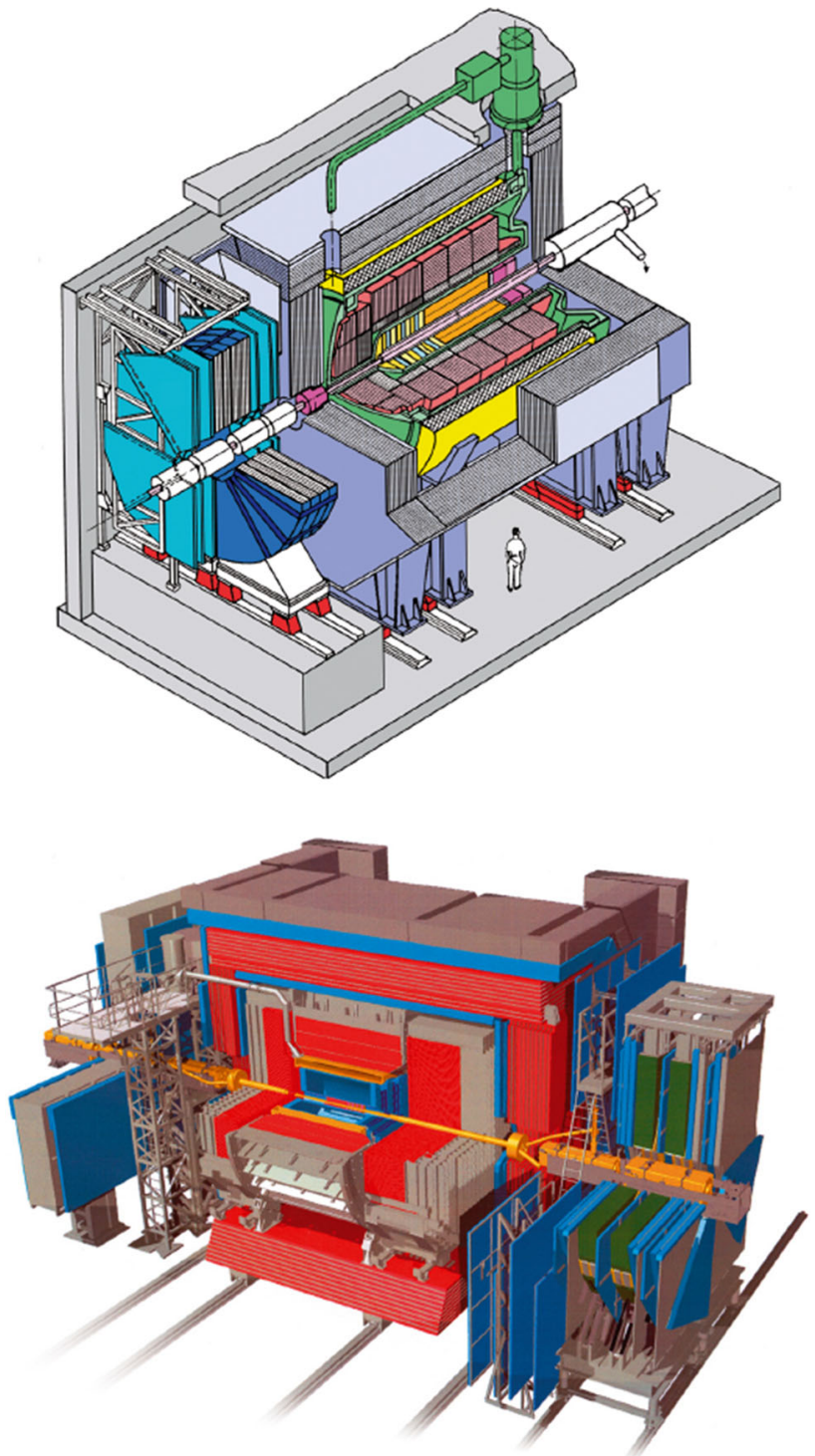

Fig. 6 The H1 (top) and ZEUS (bottom) multi-purpose detectors employed at HERA. The proton beam enters the H1 (ZEUS) detector from the right (left)

responding to the large asymmetry in the beam energies. Thus the backward region of each detector is mainly dedicated to the detection of the scattered electron, whereas the forward region contains more instrumentation and a deeper coverage. More details on the main $\mathrm{H} 1$ and ZEUS detector components are given in the following sections.

\subsection{The $\mathrm{H} 1$ detector}

The central and forward tracking detectors, which cover the regions $20^{\circ}<\theta<160^{\circ}$ and $7^{\circ}<\theta<25^{\circ}$, respectively, are used to measure charged particle trajectories and to reconstruct the interaction vertex. These detectors are enclosed together with the Liquid Argon (LAr) calorimeter inside a 
superconducting magnetic coil with a field strength of $1.16 \mathrm{~T}$ parallel to the $z$ axis. From the curvature of charged particle trajectories in the magnetic field, the central tracking system provides transverse momentum measurements with a resolution of $\sigma_{P_{T}} / P_{T}=0.002 P_{T} / \mathrm{GeV} \oplus 0.015$. The charge of the particle may also be ascertained from the direction of the curvature, and an accurate measurement of the tracks in an event provides spatial information on the interaction vertex. The central and forward tracking detectors are complemented at the very centre of the H1 detector by the central [37], backward [38] and (during HERA II only) forward silicon trackers, providing improved $z$-resolution and polar angle measurements. Additional track reconstruction is provided in the backward region by the backward drift chamber (backward proportional chamber) during the HERA I (HERA II) running period.

The LAr calorimeter [39] covers the polar angle range $4^{\circ}<\theta<154^{\circ}$ with full azimuthal $(\phi)$ acceptance and is composed of two sections: an electromagnetic calorimeter (EMC) and a hadronic calorimeter (HAC). The passive layers of the EMC are formed from $2.4 \mathrm{~mm}$ thick lead plates, whereas the HAC uses $16 \mathrm{~mm}$ thick plates of stainless steel; liquid argon forms the common sampling layer for both the EMC and the HAC. Charged particles produced in the shower ionise the argon atoms and the resulting electrons are converted to a signal and read out. The LAr is a non-compensating calorimeter, resulting in a $30 \%$ loss of the initial hadronic energy to the showering process. To account for this, an offline software technique is employed [40]. The energies of electromagnetic showers are measured in the LAr calorimeter with a precision of $\sigma(E) / E \simeq 11 \% / \sqrt{E / G e V} \oplus 1 \%$ and hadronic energy deposits with $\sigma(E) / E \simeq 50 \% / \sqrt{E / G e V} \oplus 2 \%$, as determined in test beam measurements $[41,42]$. A leadscintillating fibre calorimeter ${ }^{5}$ (SpaCal) [43] covering the backward region $153^{\circ}<\theta<178^{\circ}$ completes the measurement of charged and neutral particles. Like the LAr calorimeter, it is divided into electromagnetic and hadronic sections, although its primary function is the detection of electrons scattered through low angles. Both sections consist of scintillating fibres, which form the sampling material, embedded in a lead matrix absorber. Charged particles produced by showering in the lead cause the fibres to scintillate, and the resulting light is recorded using photomultipliers. The response to electrons is given by $\sigma(E) / E=7 \% / \sqrt{E / G e V} \oplus 1 \%$ and $\sigma(E) / E=13 \% / \sqrt{E / G e V} \oplus 4 \%$ for the electromagnetic [44] and hadronic [45] sections, respectively.

Two muon detection systems are used by H1. The return yoke of the magnetic coil is the outermost part of the detector and is equipped with limited streamer tubes (LSTs), forming

\footnotetext{
5 This device was installed in 1995, replacing a lead-scintillator sandwich calorimeter [16].
}

the central muon detector, covering the range $4^{\circ}<\theta<171^{\circ}$. When the streamer occurs within the LST, pulses generated on the strips are combined by the central muon trigger to reconstruct the muon track. In the central (barrel) region, at least 2 hits in the inner 4 layers are required. In the endcaps, which complete the iron shell around the detector, at least $3 / 5$ hits are required. The signals from the central muon system are combined with tracking information from the central tracking detector to form the muon momentum measurement. In the very forward region $\left(3^{\circ}<\theta<17^{\circ}\right)$ a set of six double layers of drift chambers, three either side of a central $1.5 \mathrm{~T}$ toroidal magnet, is used to detect muons and measure their momenta. Each layer is divided into octants and a total of 1520 drift chambers are used in the detector, varying in length from 0.40 to $2.40 \mathrm{~m}$. The field of the main $\mathrm{H} 1$ solenoid has little effect at such low $\theta$ angles and so the toroidal magnet is used to bend the path of traversing muons, enabling an independent momentum measurement.

The $\mathrm{H} 1$ detector contains approximately 270,000 readout channels, which combined with the HERA bunch crossing frequency of $96 \mathrm{~ns}$ (equivalent to an event rate of $\approx 10 \mathrm{MHz}$ ) provides a potential rate of data flow that is too high for the detector components and electronics employed to process. To cope with this, a multi-level trigger system is employed. The first level comprises triggers built using about 200 different subtriggers, each using basic information from different parts of the detector: calorimeter energies, tracks multiplicities and so on. The level one subtriggers fired by more common physics processes are only accepted by the central trigger a fraction of the time using a technique called prescaling: for a trigger with a prescale of $p$, only 1 in $p$ events are kept. Level two triggers add additional information and combine existing triggers, often utilising different subdetectors. There are two components: a topological trigger, which employs pattern recognition using a $2 \mathrm{D}$ projection (or topology) of the event in $\theta$ and $\phi$ and a neural network based system. During HERA II particle identification was employed at level three, using the Fast Track Trigger (FTT). Finally, the level four trigger runs on a filter farm and provides more detailed reconstruction and selection of tracks and clusters. The level 4 farm runs at up to $50 \mathrm{~Hz}$, at which rate the $\mathrm{H} 1$ data events are written out for analysis.

\subsection{The ZEUS detector}

Charged particles are detected by ZEUS using the Central Tracking Detector (CTD) [46-48], the Microvertex Detector (MVD) [49] and the straw-tube tracker (STT) [50]. The CTD and the MVD are located in a magnetic field of $1.43 \mathrm{~T}$, provided by a thin superconducting solenoid. The CTD consists of 72 cylindrical drift chamber layers, organised in nine superlayers covering the polar-angle region $15^{\circ}<\theta<164^{\circ}$. The MVD silicon tracker consists of a barrel (BMVD) and a 
forward (FMVD) section. The BMVD provides polar angle coverage for tracks with three measurements in the range $30^{\circ}<\theta<150^{\circ}$. The FMVD extends the polar-angle coverage in the forward region down to $7^{\circ}$. The STT covers the polar-angle region $5^{\circ}<\theta<23^{\circ}$ and consists of 48 sectors of two different sizes. Each sector, which is trapezoidal in shape and subtends an azimuthal angle of $60^{\circ}$, contains 192 (small sector) or 264 (large sector) straws of diameter $7.5 \mathrm{~mm}$, arranged into three layers. Six sectors form a superlayer, and a particle passing through the complete STT traverses eight superlayers, which are rotated around the beam direction at angles of $30^{\circ}$ or $15^{\circ}$ to each other.

A high-resolution, uranium-scintillator calorimeter (CAL) [51-54] is employed by ZEUS, consisting of three parts: the forward (FCAL), the barrel (BCAL) and the rear (RCAL) calorimeter, covering $99.7 \%$ of the solid angle around the nominal interaction point. Each part is subdivided transversely into towers and longitudinally into one electromagnetic section (EMC) and either one (RCAL) or two (BCAL and FCAL) hadronic sections (HAC). The relative energy resolutions of the CAL, as measured under test-beam conditions, are $\sigma(E) / E=18 \% / \sqrt{E / G e V}$ for electrons and $\sigma(E) / E=35 \% / \sqrt{E / G e V}$ for hadrons. The timing resolution of the CAL is better than $1 \mathrm{~ns}$ for energy deposits exceeding $4.5 \mathrm{GeV}$. Pre-sampler detectors $[55,56]$ are mounted in front of the CAL, consisting of scintillator tiles matching the calorimeter towers to measured signals from particle showers created by interactions in the material located between the interaction point and the calorimeter. The RCAL is instrumented with a layer of $3 \times 3 \mathrm{~cm}^{2}$ silicon-pad detectors at a depth of 3.3 radiation lengths forming the hadron-electron separator [57], which is used to improve the electron angle measurement.

The ZEUS muon system consists of a central barrel (BMUON) and rear (RMUON) tracking detectors [58], in addition to the forward (FMUON) tracking detectors [17]. The BMUON $\left(34^{\circ}<\theta<135^{\circ}\right)$ and RMUON $\left(135^{\circ}<\theta<\right.$ $171^{\circ}$ ) are comprised of LSTs, located behind the CAL, and inside and outside the magnetised iron yoke surrounding the CAL. The FMUON is made up of six planes of LSTs and four planes of drift chambers covering the angular region $5^{\circ}<\theta<32^{\circ}$. Whereas the central and rear muon systems use the magnetic field of the iron yoke, two iron toroids with a field strength of $1.6 \mathrm{~T}$ provide an independent muon momentum measurement in the forward direction. Muons are also detected by ZEUS in the sampling Backing Calorimeter (BAC) [59], which consists of 5200 proportional drift chambers, typically $5 \mathrm{~m}$ long with a wire spacing of $1 \mathrm{~cm}$. The $\mathrm{BAC}$ is located within the magnetised iron yoke covering the $\mathrm{CAL}$ and is equipped with analogue and digital readouts for energy and tracking measurements, respectively. The digital information from the hit wires allows the reconstruction of muon trajectories in two dimensions $(x-y$ in the barrel, $y-z$ in endcaps) with an accuracy of a few $\mathrm{mm}$.

Similarly to H1, ZEUS also employs a multi-level trigger system. The first level, which deals with the very high $10 \mathrm{MHz}$ rate, handles simple event level information, such as calorimeter energy deposits or the number of tracks in the event. At the second level more refined information is available, such as the arrival time of particles in the calorimeter, which is used to achieve an efficient separation between $e p$ collision events and background. At the third level, part of the offline reconstruction software is run in order to select signal events.

\section{Particle identification and event reconstruction}

The design and concept of the HERA detectors are driven by the physics they are required to measure and the resulting particle identification and event reconstruction methods employed by the $\mathrm{H} 1$ and ZEUS experiments are similarly defined. This section describes the identification of the particles and event level quantities used in the analyses presented in this review. A description of the various kinematic reconstruction methods, which require at least one of the components detailed below, is given in the following section.

Electrons are identified as compact, isolated energy clusters in the electromagnetic part of the calorimeters. Electron candidates are also required to have an associated track, with a distance of closest approach (DCA) to the calorimetic cluster typically less than $12 \mathrm{~cm}$. Further requirements on the electron track are often applied in the central region of the detector where coverage is more complete, such as a minimum measured transverse momentum, $P_{T}$, and a minimum radial starting position measured with respect to the nominal interaction point. The electron cluster is required to be clean, such that the energy in a cone of radius 1 in pseudorapidity ${ }^{6}$ azimuth $(\eta-\phi)$ space around the electron cluster is limited to a small fraction of the electron energy. Inefficient regions between calorimeter modules, where an electron may pass through the electromagnetic section and into the hadronic section without interaction, are excluded using fiducial volume cuts. The electron energy $E_{e}$ and polar angle $\theta_{e}$ are determined from the calorimeter cluster; the azimuthal angle $\phi_{e}$ is determined from the track. A sample of $\mathrm{NC}$ events with a well contained hadronic final state is used to perform a calibration of the electron energy, where the measured electron energy in the calorimeter, $E_{e}$, is compared to that determined via the "double angle method", $E_{D A}$, as described in Sect. 6. Further details on the electron calibration performed by $\mathrm{H} 1$ can be found in [60] and references therein.

\footnotetext{
${ }^{6}$ The pseudorapidity is defined as $\eta=-\log \tan (\theta / 2)$.
} 
Photons are identified using the same criteria as electrons concerning the isolation of the electromagnetic cluster. Conversely to electrons, however, a track veto is applied, so that any photon candidates with an associated track are rejected. For example, in the case of $\mathrm{H} 1$, a minimum track-cluster DCA of $12 \mathrm{~cm}$ and no tracks within a cone of radius $0.5 \mathrm{in} \eta$ $-\phi$ space around the cluster are required.

Muons are identified using a wide range of detector components, where the muon reconstruction algorithms require a track in the tracking system, a minimum ionising particle (m.i.p.) energy deposit in the electromagnetic and hadronic calorimeter, which is of the order of 1-2 GeV, and a signal in the outer muon detectors. Muons used in the analyses presented in this review typically have a high enough transverse momentum to traverse the tracking detectors and calorimeters, and reach the muon detectors, either central or forward, which are furthest from the interaction point. The muon momentum $P_{T}^{\mu}$ and its angular variables $\theta_{\mu}$ and $\phi_{\mu}$ are given by the associated track. Muons with a transverse momenta lower than a few $\mathrm{GeV}$ do not reach the outer muon detectors and are usually stopped in the calorimeter, but still may be identified as muons by the characteristic calorimeter pattern. Given the multi-detector nature of muon identification, a series of muon classes or grades are usually employed, depending on which information is available. These grades typically use track quality and DCA arguments, as well as requirements on the number of hit in the muon system as well as matching between the measurements available.

Jets are narrow cones of hadrons or other particles produced from the hadronisation of a quark or a gluon. In the detector, they are reconstructed as clusters of energy in the electromagnetic and hadronic calorimeter, which are recognised as coming from a collimated particle flow. Tracking information can also be used at particle momenta for which the resolution of the tracking detector is better than that of the calorimeter.

The features of jet in a hadronic final state are closely related to those of the partons originating them. However, jets are complex objects which are not uniquely defined in QCD and whose definition relies on the algorithms used to reconstruct them [61]. These come essentially in two types: cone algorithms, in which a jet is defined as a cone of radius $R$ in the $\eta-\phi$ plane, and clustering algorithms, in which particles (or energy deposits) are assigned to jets iteratively according to whether a given energy-angle resolution variable $y_{i j}$ exceeds a fixed resolution parameter $y_{\text {cut }}$. Clustering algorithms are more reliable in hadron-hadron and leptonhadron collisions, as they are not affected from ambiguities related to the presence of overlapping jets in multi-jet events.

At $\mathrm{H} 1$ and ZEUS, jets are reconstructed using the $k_{T}$ clustering algorithm [62], which uses the relative transverse momentum $k_{T}$ between the particles as a resolution variable to identify jets. This algorithm is infrared and collinear safe at any order in QCD, can be used with the same procedure both on theoretical calculations and on experimental data and treats multi-jet events without ambiguities. A jet is usually kinematically identified by its transverse energy $E_{T}^{\text {jet }}$ and its angular variables $\eta_{\text {jet }}$ and $\phi_{\text {jet }}$. Typical values used in the $k_{T}$ clustering algorithm are $R<1$ and a minimum $E_{T}^{\text {jet }}=5 \mathrm{GeV}$. Later publications [63] have also employed the anti-k $k_{T}$ algorithm; discussions on the merits of various jet clustering algorithms are available elsewhere [64].

All identified leptons are excluded from the inclusive hadronic final state and any energy around the lepton in a cone of radius of 0.5 in $\eta-\phi$ space for electrons and radius 1.0 for muons is also typically excluded. The cone is larger for muons as they tend to deposit energy in both parts of the calorimeter, whereas electrons are generally stopped by the electromagnetic section. Calibration of the hadronic final state is performed using a large NC sample by comparing the transverse momentum of the calibrated electron, $P_{T}^{e}$ to that of the inclusive hadronic final state, $P_{T}^{h}$. The $P_{T}$ balance, $P_{T}^{h} / P_{T}^{e}$ should be equal to 1 in intrinsically balanced $\mathrm{NC}$ events and the data are adjusted iteratively until they are in agreement with the MC simulation. The calibration procedure may involve additional steps, in particular in the treatment of jets; see for example [36].

The missing transverse momentum, $P_{T}^{\text {miss }}$, is calculated using the vector sum of all identified particles and a significant value of this quantity may indicate the presence of a neutrino, or neutrinos, in the event. The vector $\mathbf{p}_{T}^{\text {miss }}$ is derived from the total visible hadronic momentum vector, $\mathbf{p}_{T}$, by $\mathbf{p}_{T}^{\text {miss }}=-\mathbf{p}_{T}$, where

$$
\begin{aligned}
\mathbf{p}_{T} & =\left(P_{x}, P_{y}\right) \\
& =\left(\sum_{i} E_{i} \sin \theta_{i} \cos \phi_{i}, \sum_{i} E_{i} \sin \theta_{i} \sin \phi_{i}\right) .
\end{aligned}
$$

For example, in CC events a significant imbalance of transverse momenta of measured final-state particles is observed. As a consequence, the $P_{x}$ and $P_{y}$ components of $\mathbf{p}_{T}^{\text {miss }}$ are non zero, and they can be attributed to the outgoing neutrino. Many of the final states examined in this review feature neutrinos, and as such $P_{T}^{\text {miss }}$ is employed in such analyses. A reliable measurement of this quantity is made possible via the near hermetic coverage of the $\mathrm{H} 1$ and ZEUS detectors around their respective interactions points. A related quantity used in several $\mathrm{H} 1$ analyses is $P_{T}^{\text {calo }}$, the net transverse momentum calculated from all reconstructed particles measured in the calorimeter. This quantity reflects the missing transverse momentum as seen by the trigger. For events containing highenergy muons, where only the (relatively small) muon energy deposited in the calorimeter is included, $P_{T}^{\text {calo }} \simeq P_{T}^{h}$, otherwise $P_{T}^{\text {calo }}=P_{T}^{\text {miss }}$. 


\section{Kinematic reconstruction methods}

The reconstruction of the scattered electron together with that of the hadronic final state is of particular importance at HERA as it allows the determination of the kinematic variables introduced in Sect. 1. Indeed, one of the salient features of the $\mathrm{H} 1$ and ZEUS experiments is the possibility to determine $\mathrm{NC}$ event kinematics from the scattered electron or from the hadronic final state, or using a combination of the two. In the case of NC DIS, exclusively using the scattered electron to determine the event kinematics results in a less model-dependent analysis, which is easier to interpret theoretically. Resolution, measurement accuracy and effects due to the radiation of photons by the incoming electron influence the choice of the reconstruction method in a given kinematic region. For a broader discussion of the reconstruction methods presented in this section, see for example [60,65].

For NC scattering, the "electron method" is favoured, where the inelasticity and the negative four-momentum transfer squared are calculated from the scattered electron energy $E_{e}$ and polar angle $\theta_{e}$ :

$Q_{e}^{2}=\frac{P_{T}^{e 2}}{1-y_{e}}, \quad y_{e}=1-\frac{\Sigma_{e}}{2 E_{e}^{0}}, \quad x_{e}=\frac{Q_{e}^{2}}{s y_{e}}$,

where $P_{T}^{e}=E_{e} \sin \theta_{e}$ is the electron transverse momentum, $\Sigma_{e}=E_{e}\left(1-\cos \theta_{e}\right)=E_{e}-P_{z}^{e}$, and $E_{e}^{0}$ is the energy of the initial-state electron. Using this method, the negative four-momentum transfer squared may also be expressed as

$Q_{e}^{2}=4 E_{e}^{0} E_{e} \cos ^{2} \frac{\theta_{e}}{2}$

In the case of $\mathrm{CC}$ events, as the scattered neutrino is not detected only the information on the hadronic final state can be used for the reconstruction of the event kinematics. The "hadron method" or "Jacquet-Blondel method" [66] uses similar relations to those defined in Eq. (9a), obtained exclusively from the reconstructed hadronic final state:

$Q_{h}^{2}=\frac{P_{T}^{h^{2}}}{1-y_{h}}, \quad y_{h}=\frac{\Sigma_{h}}{2 E_{e}^{0}}, \quad x_{h}=\frac{Q_{h}^{2}}{s y_{h}}$,

where $\Sigma_{h}=\sum_{i}\left(E_{i}^{h}-p_{z, i}^{h}\right)$ is the total hadronic $E_{h}-P_{z}^{h}$, summed over all reconstructed hadronic final-state particles $i$, and $P_{T}^{h}$ is transverse momentum of the inclusive final state. A combination of $P_{T}^{h}$ and $\Sigma_{h}$ defines the hadronic scattering angle, $\gamma_{h}$, where

$\tan \frac{\gamma_{h}}{2}=\frac{\Sigma_{h}}{P_{T}^{h}}$

which, within the Quark Parton Model (QPM) [67] corresponds to the direction of the struck quark.
The "sigma method" [68] makes use of both electron and hadronic final-state variables and Eqs. (9a) and (10) are modified as

$Q_{\Sigma}^{2}=\frac{P_{T, e}^{2}}{1-y_{\Sigma}}, \quad y_{\Sigma}=\frac{\Sigma_{h}}{E-P_{z}}, \quad x_{\Sigma}=\frac{Q_{\Sigma}^{2}}{s y_{\Sigma}}$

The total $E-P_{z}$ of the event, $\delta$, is defined as

$$
\begin{aligned}
\delta \equiv E-P_{z} & =E_{e}\left(1-\cos \theta_{e}\right)+\sum_{i}\left(E_{i}^{h}-p_{z, i}^{h}\right) \\
& =\Sigma_{e}+\Sigma_{h}
\end{aligned}
$$

and for events with no photon radiation from the incoming electron $\delta=2 E_{e}^{0}=55 \mathrm{GeV}$.

The "e-sigma method" [69] is most often used for the reconstruction of NC kinematics and is an optimum combination of the two methods, providing good resolution whilst minimising radiative effects [70]:

$Q_{e \Sigma}^{2}=Q_{e}^{2}, \quad y_{e \Sigma}=\frac{Q_{e}^{2}}{s x_{\Sigma}}, \quad x_{e \Sigma}=x_{\Sigma}$.

Finally, the "double angle method" [71,72] is used to reconstruct the event kinematics from the electron and hadronic scattering angles:

$$
\begin{aligned}
y_{D A} & =\frac{\tan \left(\gamma_{h} / 2\right)}{\tan \left(\theta_{e} / 2\right)+\tan \left(\gamma_{h} / 2\right)}, \\
Q_{D A}^{2} & =4 E_{e}^{0^{2}} \cdot \frac{\cot \left(\theta_{e} / 2\right)}{\tan \left(\theta_{e} / 2\right)+\tan \left(\gamma_{h} / 2\right)}, \\
x_{D A} & =\frac{Q_{D A}^{2}}{s y_{D A}} .
\end{aligned}
$$

In this method, the energy of the scattered electron may be calculated via

$E_{D A}=\frac{2 E_{e}^{0} \sin \gamma_{h}}{\sin \gamma_{h}+\sin \theta_{e}-\sin \left(\gamma_{h}+\theta_{e}\right)}$,

which is used in the calibration of the electron energy as described in Sect. 5. This method is largely insensitive to hadronisation and is, to first order, independent of the detector energy scales. However, the hadronic angle is less well determined than the electron angle due to particle loss in the beam pipe, and an additional correction may be applied [65].

\section{Physics simulation}

To study different physics models and in particular compare these models with experimental data, stochastic techniques are employed. These techniques, which use random numbers and probability distributions, are termed Monte Carlo (MC) methods. Simulation is an essential tool for physics analysis, 
contributing to a better understanding of the data and of the detector response to physics events. Moreover, the theoretical models implemented in MC programs may be tested by comparing the prediction from the simulation to what is observed in the real data. The simulation of physics events at HERA, much like at other particle physics colliders, can be broken down into three discrete steps: event generation, detector simulation, and finally reconstruction of the simulated events. These steps are briefly described in the following.

\subsection{Event generation}

Firstly, QCD MC event generators are used which employ the factorisation theorem [73] to describe the ep hard scatter, characterised by an associated scale allowing the collision to be factorised into separate stages. Event generators produce all final-state partons for a given interaction, using all relevant diagrams and parton density functions. The hard sub-process is the main feature of the event, and is the interaction of a parton extracted from the proton and the photon (or a photon constituent in resolved photon events). This process can be calculated in a fixed-order perturbative expansion since it involves a hard scale $\mu$. Hadronisation is the process in which colourless hadrons are formed starting from coloured partons produced in the hard scatter. It is a non-perturbative phenomenon which is modelled by the simulation programs using phenomenological inputs. The main hadronisation models available include the cluster model (for example, as done by HERWIG [74]) and the Lund string model [75] (for example, as done by PYTHIA [76] and JETSET [77]). In processes involving charged and coloured objects the topology of an event can be strongly influenced by the emission of gluons and photons by the initial or final state. These perturbative corrections are usually modelled by the so-called parton shower method, where the radiation is simulated by an arbitrary number of branchings of one parton into two, such as $e \rightarrow e \gamma, q \rightarrow q g, q \rightarrow q \gamma$ or $g \rightarrow q \bar{q}$. A final consideration is the beam remnant, which comprises the remainder of the initial-state particles, following the hard scatter and any initial and/or final-state radiation. If this remnant is coloured, it will be necessarily connected to the rest of the event and needs to be fragmented and reconstructed coherently. The interactions of any unstable partons produced (mainly quarks and gluons) are further simulated until only long lived stable particles exist. The simulated event then consists of a list of 4-vectors, describing the final-state particles.

\subsection{Detector simulation}

The output of the event generator comprises a list of particles produced in the hard scattering, as well as the particles produced from the parton shower, as explained in the previous section. In real data the only available information from an electron-proton scattering is the signal the particles produced in the collision leave in the various detector sub-components as they pass through the detector. A full detector simulation is therefore performed in order to also describe this at the MC level. The passage of particles through the detector is simulated with the GEANT3 [78] package. GEANT provides a description of all detector components, including the composite material, as well as the shapes and relative positions. The program traces the passage of a particle through the whole detector, simulating its response whilst taking into account the relevant physics processes such as energy loss, multiple scattering and particle decays in flight. After the detector response has been simulated, the trigger logic as implemented in the data taking is added to the simulation. The simulated event now resembles a set of hits on wires, energy deposits in the calorimeters, signals in the muons chambers and so on, mimicking the traces left in the detector by a real ep collision event.

\subsection{Reconstruction of simulated events}

As a final step, the same reconstruction program used for the data is applied to simulated events. This program reconstructs the event variables, like particle momenta and energies, treating the data and the Monte Carlo in the same way. All the information coming from the different detector subcomponents are taken as input by the reconstruction program. MC simulated data are thus identical to real data, with the addition of the generator level information. This information and the difference between the two levels of simulation also provide a method of correcting detector acceptances and resolution effects in the data.

\section{Standard Model physics at HERA}

The cross sections of inclusive NC and CC interactions, which are measured at HERA with high precision, are one of the most important ingredients for the determination of the proton parton distribution functions. The kinematic region in $x$ and $Q^{2}$ covered by the HERA experiments is shown in Fig. 2 and comprises a significant part of the $x$ region of interest for the LHC experiments. As a consequence, taking advantage of QCD factorisation and the use of DGLAP equations [10-12,79,80], the PDFs extracted from the HERA data can be used as input for cross section determination at the LHC.

The H1 and ZEUS Collaborations published [81] a combination of their NC and CC cross section measurements extracted from the full data sample collected at HERA. The details of how the combination was performed, in particular regarding the treatment of the systematic uncertainties, are described in detail elsewhere [81]. For the aims of this 
paper it is sufficient to say that the cross sections measured by the two collaborations are combined using a $\chi^{2}$ minimisation method, which takes into account both the statistical and systematic uncertainties of the data. In particular, a distinction is made between the correlated and uncorrelated uncertainties among the different points (bins) of the analysis. In addition to the clear improvement on the statistical uncertainty obtained by combining the data, an improvement on the systematics uncertainties is also obtained. Intuitively, correlated systematic uncertainties that affect the measurement in one direction for $\mathrm{H} 1$, and in the other for ZEUS, can be significantly reduced in the data combination. The fact that the detectors and the analysis techniques are different is fully exploited, where these differences translate into systematic uncertainties affecting the data in a different way. In this sense, the detectors are used to "cross calibrate" each other. These arguments are explained in full mathematical detail in the papers illustrating the method employed $[82,83]$. It is also worthwhile pointing out also that this method allows the consistency of the data of the two experiments to be checked in a model-independent way, as the main assumption done in the data combination is that there is a single true value of the cross section corresponding to each data point and each process, $\mathrm{NC}$ or $\mathrm{CC} e^{+} p$ or $e^{-} p$ scattering.

\subsection{Neutral current measurements}

Cross sections for NC DIS interactions have been published [81] for $0.045 \leq Q^{2} \leq 50000 \mathrm{GeV}^{2}$, in a large phase-space region $6 \cdot 10^{-7} \leq x \leq 0.65$ for values of inelasticity $0.005 \leq y \leq 0.95$ (see Fig. 2). Covering the very low $Q^{2}$ regions required special experimental techniques. The lowest- $Q^{2}$ data, $Q^{2}>0.045 \mathrm{GeV}^{2}$, were collected during the HERA I data taking period with the ZEUS detector using special tagging devices [84]. The $Q^{2}$ range between $0.2 \mathrm{GeV}^{2}$ and $1.5 \mathrm{GeV}^{2}$ was covered using special HERA I runs, in which the interaction vertex position was shifted forward, bringing backward-scattered electrons with small scattering angles into the acceptance of the detectors [82, 85,86]. The $Q^{2}>1.5 \mathrm{GeV}^{2}$ region was covered with HERA I and HERA II data in different configurations.

In performing the analyses, three main different $Q^{2}$ regions were considered, as the analysis methods differ substantially among them. At $\sqrt{s}=318 \mathrm{GeV}$, the high $Q^{2}$ region is defined for $Q^{2}$ between 150 and $30000 \mathrm{GeV}^{2}$, whereas the low $Q^{2}$ region comprises the range $2<Q^{2}<$ $120 \mathrm{GeV}^{2}$. The very-low $Q^{2}$ region, populated only with data collected at $\sqrt{s} \leq 300 \mathrm{GeV}$, is defined for $Q^{2}<2 \mathrm{GeV}^{2}$. As a breakdown of perturbative QCD (pqCD) is expected for $Q^{2}$ approaching $1 \mathrm{GeV}^{2}$, the data of this last region cannot be compared to predictions from $\mathrm{pQCD}$.
The most interesting region for BSM searches is the high $Q^{2}$ region, in particular at very low $x$, where the cross section is lower and the precision of the measurements is worse so that new phenomena can hide in the large SM background. In general, searches for deviations from the SM are performed at the limits of the accessible kinematic regions. Therefore, the analysis for the high $Q^{2}$ analysis is reported here as representative. The selection techniques of the many other analyses included in the combined data are described in detail elsewhere ([81] and references therein).

NC DIS events are generated with the DJANGOH [87] MC simulation program, which is based on HERACLES [88] for the electroweak calculation and LEPTO [89] for the hard matrix element calculation. The colour dipole model as implemented in ARIADNE [90] is used to generate higherorder QCD dynamics. The JETSET program is used to simulate the hadronisation process in the 'string-fragmentation' model.

Events are selected by requiring the DIS electron to be reconstructed in the final state. The electron reconstruction is based on an algorithm combining information from the calorimeter energy deposits and tracks measured in the central tracking detectors. The electron is required to have an energy $E_{e}^{\prime}>10 \mathrm{GeV}$ and to be isolated from other energy deposits in the calorimeter. If the electron is found in the acceptance region of the tracking detectors, a track matched to the energy deposit in the calorimeter is required. The matching is performed considering the distance of closest approach between the track extrapolated to the calorimeter surface and the energy cluster position. A matched track is not required if the electron emerged outside the acceptance of the tracking detectors.

The most important background in the NC DIS analysis comes from photoproduction events, when an energy deposit in the calorimeter associated to a charged track is wrongly identified as the scattered DIS electron. In order to suppress this background, a cut on the total $E-P_{Z}$ as defined in Eq. (13) is used in the event selection, by requiring events to have $38<\delta<65 \mathrm{GeV}$. As already mentioned in Sect. 6, conservation of energy and longitudinal momentum implies that $\delta=2 E_{e}=55 \mathrm{GeV}$, if all final-state particles are detected and perfectly measured. Undetected particles that escape down the forward beam pipe have a negligible effect on $\delta$. However, particles lost down the backward beam pipe could lead to a substantial reduction in $\delta$. This is the case for photoproduction events, where the electron emerges at a very small scattering angle, or for events in which an initial-state bremsstrahlung photon is emitted. In order to further reduce the photoproduction background, the selected NC events are required to have $y<0.9$. For the puposes of this review, it is worth noting that this requirement cuts out part of the kinematic region interesting for BSM searches. 


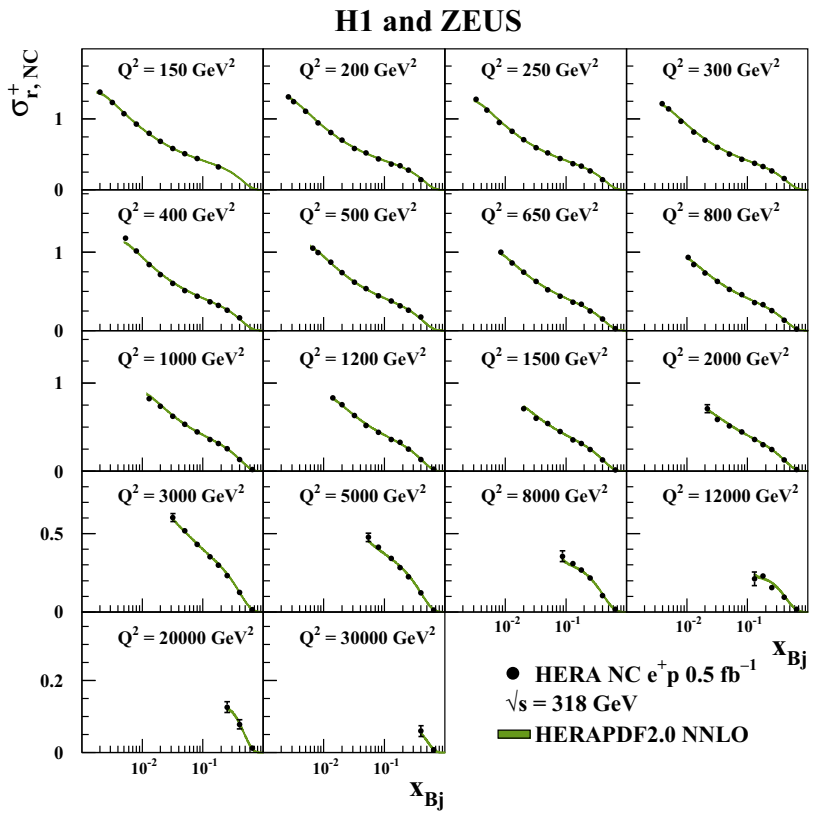

Fig. 7 The combined high $Q^{2}$ HERA inclusive $\mathrm{NC} e^{+} p$ reduced cross sections at $\sqrt{s}=318 \mathrm{GeV}$, plotted as a function of $x$ at fixed $Q^{2}$, with overlaid predictions of the HERAPDF2.0 NNLO. The bands represent the total uncertainties on the predictions

Further cuts are applied in order to suppress the backgrounds from beam-gas and cosmic interactions. After all cuts, the remaining background contamination estimated using photoproduction $\mathrm{MC}$ is about $0.2 \%$.

Cross sections are extracted and compared to theoretical predictions from $\mathrm{pQCD}$, based on the PDF set HERAPDF2.0, which will be introduced in Sect. 8.4. The reduced cross section $\tilde{\sigma}_{N C}^{ \pm}$is defined in terms of the inclusive NC cross section as

$\sigma_{\mathrm{r}, \mathrm{NC}}^{ \pm}=\frac{\mathrm{d}^{2} \sigma\left(e^{ \pm} p\right)}{\mathrm{d} x \mathrm{~d} Q^{2}} \cdot \frac{Q^{4} x}{2 \pi \alpha^{2} Y_{+}}$

where the fine-structure constant, $\alpha$, is defined at scale zero and $Y_{ \pm}=1 \pm(1-y)^{2}$. The combined high $Q^{2}$ inclusive NC $e^{+} p$ reduced cross sections at $\sqrt{s}=318 \mathrm{GeV}$, as extracted from the combined HERA data, are shown in Fig. 7. The cross sections are shown as a function of $x$ in different $Q^{2}$ bins and are compared to the HERAPDF2.0 predictions [81] at nextto-next-to-leading (NNLO). The combined low $Q^{2}$ HERA inclusive $\mathrm{NC} e^{+} p$ reduced cross sections at $\sqrt{s}=318 \mathrm{GeV}$ are shown in Fig. 8. The data description by the SM prediction is generally good, except for the turnover of the cross section at low $x$ and low $Q^{2}$.

The combined reduced cross section as a function of $Q^{2}$ at different $x$ values is shown in Fig. 9. In the high $Q^{2}$ region, the $e^{-} p$ cross section is significantly larger than the $e^{+} p$. This is due to the parity-violating component of the NC interactions, namely to the exchange of a $Z^{0}$ boson between the electron and the proton. This component is suppressed at low $Q^{2}$ due

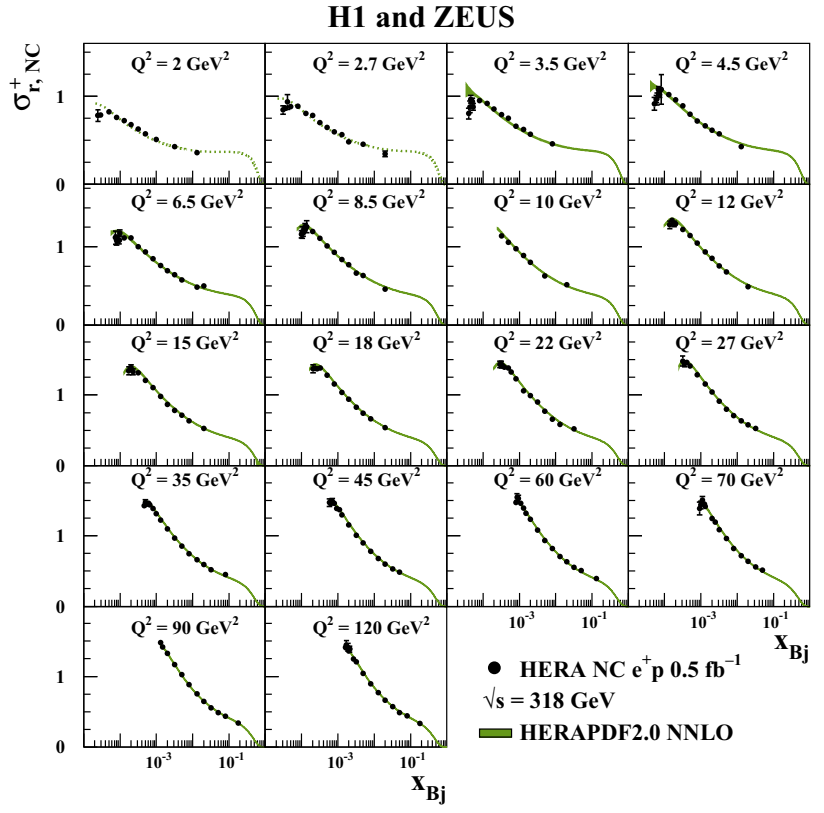

Fig. 8 The combined low $Q^{2}$ HERA inclusive $\mathrm{NC} e^{+} p$ reduced cross sections at $\sqrt{s}=318 \mathrm{GeV}$, plotted as a function of $x$ at fixed $Q^{2}$, with overlaid predictions of the HERAPDF2.0 at NNLO order in pQCD. The bands represent the total uncertainties on the predictions. Dotted lines indicate extrapolation into kinematic regions not included in the fit

to the large mass of the $Z^{0}$ boson and becomes relevant only for $Q^{2} \sim M_{Z^{0}}^{2}$.

In Fig. 9 the HERA data are compared to the results of fixed-target experiments, which populate the region of lower $Q^{2}$ and higher $x$ values. The HERA and the fixed-target results are in good agreement, as shown by the comparison with theoretical predictions based on the HERA data alone [81]. The level of agreement between the two fixedtarget experiment results shown in the figure [91,92] was investigated in [92] and found to be good, taking into account the normalisation uncertainties of the data and the systematic uncertainties quoted in the analyses. The HERA data significantly extend the region where the reduced cross section can be measured with very good precision.

Scaling violations, as predicted by the DGLAP equations, are also clearly visible in Fig. 9, where in the low $x$ region the reduced cross section is not independent of $Q^{2}$, but shows a slope that becomes steeper with decreasing $x$. These violations are due to QCD effects not present in the naïve QPM model. The large kinematic range covered by HERA clearly demonstrates the scaling violations and allows them to be used for the extraction of the gluon density in the proton. On the other hand, it has to be noted that the gluon content of the proton cannot be measured directly at HERA. This means that phenomenological assumptions are needed in the extraction of the gluon density from the data, introducing an important source of uncertainty in the extraction of the proton PDFs. 


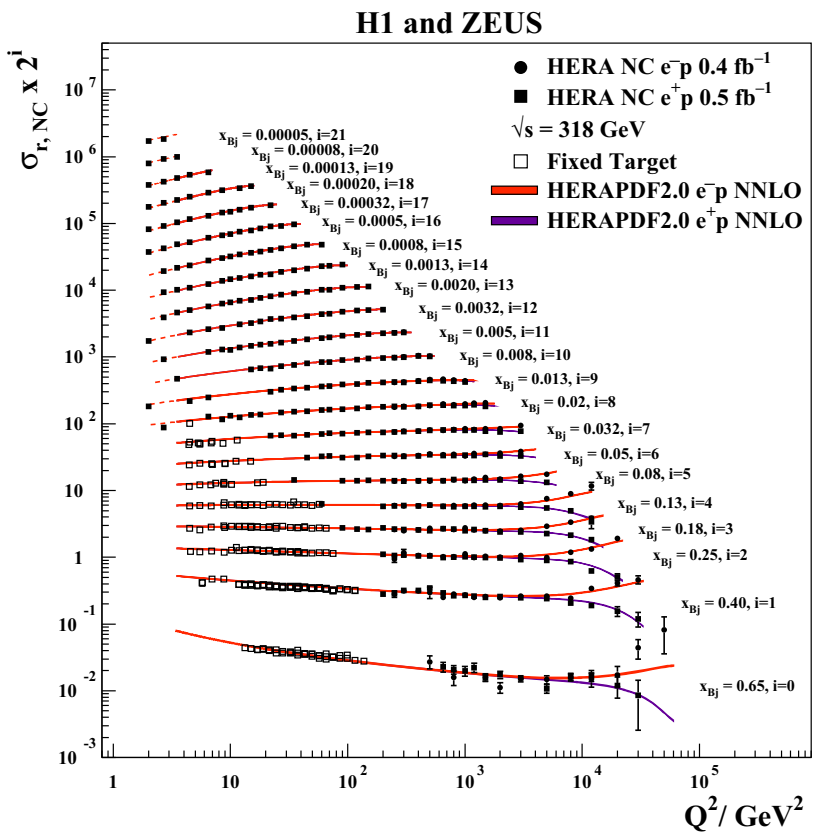

Fig. 9 The combined HERA data for the inclusive $\mathrm{NC} e^{+} p$ and $e^{-} p$ reduced cross sections together with fixed-target data $[91,92]$ and the predictions of HERAPDF2.0 NNLO [81]. The bands represent the total uncertainty on the predictions

The HERA inclusive NC DIS cross sections are an important input to the determination of the proton structure functions. The double-differential cross section of the electronproton NC DIS as a function of $x$ and $Q^{2}$ can be expressed in terms of the proton structure functions $F_{2}, F_{3}$ and $F_{L}$, as [81]:

$\frac{\mathrm{d}^{2} \sigma\left(e^{ \pm} p\right)}{\mathrm{d} x \mathrm{~d} Q^{2}}=\frac{2 \pi \alpha^{2}}{x Q^{4}}\left\{Y_{+} F_{2} \mp Y_{-} x F_{3}-y^{2} F_{L}\right\}$.

It follows that from the reduced cross section, $\tilde{\sigma}_{N C}^{ \pm}$, the proton structure functions can be constrained:

$\sigma_{\mathrm{r}, \mathrm{NC}}^{ \pm}=F_{2} \mp \frac{Y_{-}}{Y_{+}} x F_{3}-\frac{y^{2}}{Y_{+}} F_{L}$.

The structure functions $F_{2}, F_{3}$ and $F_{L}$ are process dependent. $F_{3}$ is non-zero only for weak interactions and is generated by parity-violating interactions, i.e. by the exchange of a $W$ boson in CC DIS or a $Z^{0}$ boson in NC DIS between the electron and the proton. Therefore, for $Q^{2} \ll M_{Z^{0}}^{2}$ :

$\sigma_{\mathrm{r}, \mathrm{NC}}^{ \pm}=F_{2} \mp-\frac{y^{2}}{Y_{+}} F_{L}$

The $F_{L}$ term is related to the longitudinally polarised virtual boson exchange process. This term vanishes at lowestorder QCD but has been predicted [93] to be non-zero when including higher-order QCD terms. The contribution of $F_{L}$ to the reduced NC cross section is relevant only for values of $y$ larger than approximately 0.5 .

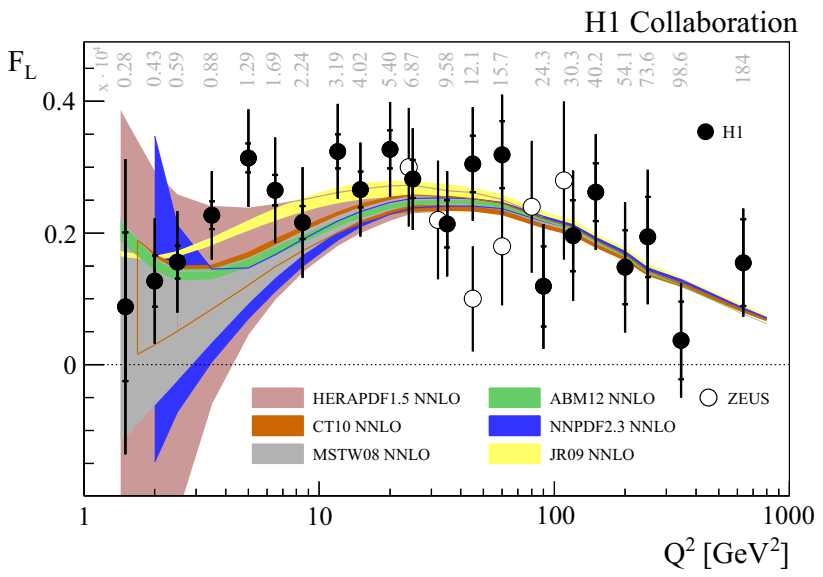

Fig. 10 The proton structure function $F_{L}$ averaged over $x$ at different $Q^{2}$ (solid points). The average value of $x$ for each $Q^{2}$ is given above each data point. The inner error bars represent the statistical uncertainties, the full error bars include the statistical and systematic uncertainties added in quadrature, including all correlated and uncorrelated uncertainties. The data are compared to NNLO predictions from a selection of PDF sets as indicated ([96] and references therein)

A direct measurement of $F_{L}$ has been performed at HERA by determining the reduced cross section, $\sigma_{\mathrm{r}, \mathrm{NC}}^{ \pm}$, at different values of $\sqrt{s}$ by reducing the proton-beam energy from $E_{p}=920 \mathrm{GeV}$ to $E_{p}=460 \mathrm{GeV}$ and $E_{p}=575 \mathrm{GeV}$, as described in Sect. 3. This method had been previously used to extract $F_{L}$ in fixed-target experiments [91,92,94,95]. From Eq. (6), $y=Q^{2} / s x$ and therefore the cross sections can be measured at the same values of $x$ and $Q^{2}$ but at different values of $y$, allowing an experimental separation between $F_{2}$ and $F_{L}$ in Eq. (19). The sensitivity to $F_{L}$ is increased by measuring the cross sections in the high $y$ region, but in this region the electron energy is low and the background from photoproduction is large. The separation between NC DIS and the photoproduction background is therefore one of the main challenges of the high $y$ analysis.

The structure function $F_{L}$ as measured by the $\mathrm{H} 1$ [96] and ZEUS [97] Collaborations is shown in Fig. 10. The data are compared with several QCD predictions at NNLO, which describe the measurements reasonably well.

A precise direct measurement of $F_{L}$ would allow, according to the Altarelli-Marinelli relation [93], a direct extraction of the gluon density in the proton, which is constrained indirectly via scaling violations. The $F_{L}$ measurement was used [96] to perform a gluon density extraction based on a NLO approximation, and the agreement with the gluon as determined from scaling violations was found to be reasonably good. Although the scaling violations allow a much better constraint of the gluon in the proton, the comparison with the direct extraction from the $F_{L}$ measurement provides an independent check of its validity. Although more at a qualitative than at a quantitative level, this measurement represents 
an improvement on the knowledge of the gluon density in the proton.

From Eq. (18) it follows that the structure function $x F_{3}$ can be extracted from the difference between the $e^{+} p$ and $e^{-} p$ reduced cross sections:

$x F_{3}=\frac{Y_{+}}{2 Y_{-}}\left(\sigma_{\mathrm{r}, \mathrm{NC}}^{-}-\sigma_{\mathrm{r}, \mathrm{NC}}^{+}\right)$.

It is useful to consider the simplified picture as expressed in the QPM, where $F_{L}=0$. In the QPM, $x F_{3}$ is directly related to the valence quark distributions in the proton, which, assuming symmetry between the quarks and anti-quarks in the sea, can be expressed as

$x u_{v}=x U-x \bar{U}$,

$x d_{v}=x D-x \bar{D}$

where $x U, x \bar{U}, x D$ and $x \bar{D}$ represent the sums of parton distributions for up-type and down-type quarks and antiquarks, respectively. Below the $b$-quark mass threshold, they are related to the quark distributions as follows:

$x U=x u+x c, \quad x \bar{U}=x \bar{u}+x \bar{c}$,

$x D=x d+x s, \quad x \bar{D}=x \bar{d}+x \bar{s}$,

where $x s$ and $x c$ are the strange- and charm-quark distributions.

In the HERA kinematic regime the dominant contribution to $x F_{3}$ comes from its photon- $Z^{0}$ exchange interference term, $x F_{3}^{\gamma Z}$. In the QPM, this is expressed by the relation:

$x F_{3}^{\gamma Z} \approx \frac{x}{3}\left(2 u_{v}+d_{v}\right)$,

which shows that $x F_{3}$ is directly related to the valence quark distributions in the proton. The measurements of $x F_{3}^{\gamma Z}$ therefore make it possible to determine the behaviour of the valence quark distributions at low $x$. The structure function $x F_{3}^{\gamma Z}$ as extracted from the HERA combined NC DIS cross sections is shown in Fig. 11 and compared to pQCD predictions from HERAPDF2.0.

\subsection{Charged current measurements}

The CC DIS process $e p \rightarrow v X$ was measured at HERA for the first time in 1993. The $Q^{2}$ dependence of the CC DIS cross section is sensitive to the $W$ boson mass, which enters the $\mathrm{CC}$ propagator. One of the aims of the first measurements of $\mathrm{CC}$ DIS was the determination of this mass, which was extracted from a fit to the differential CC DIS cross section as a function of $Q^{2}$, leaving it as a free parameter of the fit. The fact that the obtained value was consistent to the mass of the $W$ boson as measured by the hadron colliders at that time demonstrated the presence of the $W$ propagator [98,99]. The measurement of CC DIS interactions in ep collisions, $e^{+} p \rightarrow \bar{v} X\left(e^{-} p \rightarrow\right.$ $v X)$, provides a complementary view with respect to NC DIS

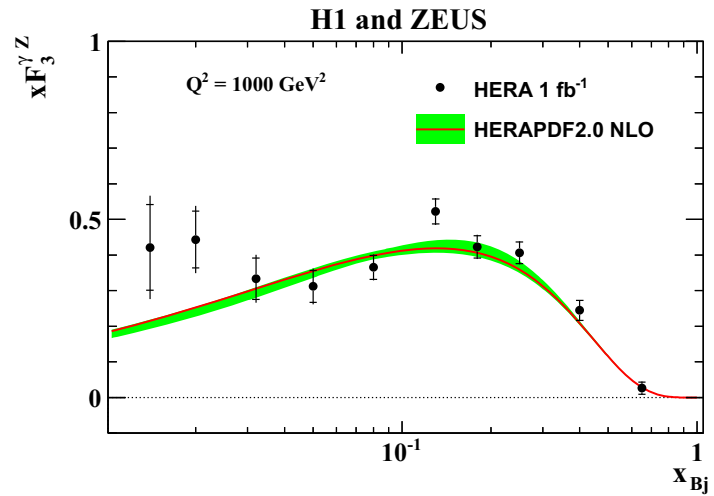

Fig. 11 The structure function $x F_{3}^{\gamma Z}$ averaged over $Q^{2} \geq 1000 \mathrm{GeV}^{2}$ at the scale $Q^{2}=1000 \mathrm{GeV}^{2}$ together with the prediction from HERAPDF2.0 NLO. The band represents the total uncertainty on the prediction

for the understanding of the proton structure and the SM. While NC DIS is mediated by the exchange of photons and $Z^{0}$ bosons and is sensitive to all quark flavours, only downtype quarks and up-type anti-quarks (down-type anti-quarks and up-type quarks) contribute at leading order to $e^{+} p\left(e^{-} p\right)$ CC DIS. Thus this process is a powerful probe of flavourspecific PDFs, as described in more detail in the following. Although part of this information could already be obtained from the previous fixed-target neutrino experiments, NC and CC DIS became accessible at the same machine for the first time at HERA, and in an extended kinematic region.

The precision of the measurement of the $\mathrm{CC}$ cross sections at HERA has been significantly improved by combining the data of the two experiments $\mathrm{H} 1$ and ZEUS. Cross sections for $\mathrm{CC}$ interactions have been published [81] for $200 \leq Q^{2} \leq$ $50000 \mathrm{GeV}^{2}$ and $1.3 \cdot 10^{-2} \leq x \leq 0.40$ at values of $y$ between 0.037 and 0.76 .

As described for $\mathrm{NC}$ in the previous section, CC DIS events are generated using the DJANGOH MC simulation program, interfaced with LEPTO for the hard matrix calculation and HERACLES for electroweak effects. A set of NC DIS events generated with DJANGOH is used to estimate the NC contamination in the CC sample while the photoproduction background was simulated using HERWIG or PYTHIA. The GRAPE [100] and EPVEC [101] MC programs are used to simulate the background from lepton pair and single $W$ boson production, respectively.

In CC DIS, the struck quark gives rise to one or more jets of hadrons and the energetic final-state neutrino escapes detection, leaving a large imbalance in the transverse momentum observed in the detector. Therefore, CC DIS events are selected by requiring a large missing transverse momentum in the event, $P_{T}^{\text {miss }}$, as defined in Eq. (8).

Backgrounds to CC DIS arise from high $E_{T}$ events in which the finite energy resolution of the calorimeter or energy that escapes detection leads to significant missing transverse 
momentum. Non-ep events such as beam-gas interactions, beam-halo muons or cosmic rays can also cause substantial imbalance in the measured transverse momentum and constitute additional sources of background. Moreover, single $W$ boson production events and di-lepton events could also constitute a potential background in case the lepton(s) are poorly reconstructed.

$\mathrm{CC}$ events are selected in the kinematic region $Q^{2}>$ $200 \mathrm{GeV}^{2}$ and $y<0.9$, with large missing transverse momentum, $P_{T}^{\text {miss }}>12 \mathrm{GeV}$, and a primary vertex reconstructed in the nominal interaction region. The vertex requirement significantly reduces the non-ep background. Background from $\mathrm{NC}$ events with a poorly measured scattered electron or hadronic jets, leading to significant missing transverse momentum, are removed by rejecting events with an isolated electromagnetic cluster in the calorimeter and a longitudinal balance $\delta>30 \mathrm{GeV}$. Other cleaning cuts are used to reduce the remaining backgrounds, based on for example the number of tracks fitted to the vertex compared to the total, or on the azimuthal collimation of the energy flow in the event, using the ratio $V_{\mathrm{ap}} / V_{\mathrm{p}}$ [102], where $V_{\mathrm{p}}$ and $V_{\mathrm{ap}}$ are the transverse energy flow parallel and antiparallel to the hadronic final state $\mathbf{p}_{T}^{h}$, respectively, and are determined from the transverse momentum vectors $\mathbf{p}_{T}^{i}$ of all the particles $i$ which belong to the hadronic final state according to:

$$
\begin{gathered}
V_{\mathrm{p}}=\sum_{i} \frac{\mathbf{p}_{T}^{h} \cdot \mathbf{p}_{T}^{i}}{P_{T}^{h}} \quad \text { for } \mathbf{p}_{T}^{h} \cdot \mathbf{p}_{T}^{i}>0 \\
V_{\mathrm{ap}}=\sum_{i} \frac{\mathbf{p}_{T}^{h} \cdot \mathbf{p}_{T}^{i}}{P_{T}^{h}} \quad \text { for } \mathbf{p}_{T}^{h} \cdot \mathbf{p}_{T}^{i}<0
\end{gathered}
$$

After all cuts, the contamination from NC DIS and non$e p$ events is found to be negligible, while other backgrounds from photoproduction, single $W$ boson production and lepton pairs contribute up to $20 \%$ in the lowest- $Q^{2}$ and lowest $x$ bins. One of the selected CC events, as seen by the ZEUS detector, is shown in Fig. 12.

Similarly to NC, cross sections are extracted and compared to the theoretical predictions from $\mathrm{pQCD}$, based on HERAPDF2.0. The reduced cross sections for unpolarised $\mathrm{CC} e^{ \pm} p$ scattering are defined as:

$\sigma_{r, \mathrm{CC}}^{ \pm}=\frac{2 \pi x}{G_{F}^{2}}\left[\frac{M_{W}^{2}+Q^{2}}{M_{W}^{2}}\right]^{2} \frac{\mathrm{d}^{2} \sigma_{\mathrm{CC}}^{e^{ \pm} p}}{\mathrm{~d} x \mathrm{~d} Q^{2}}$.

The combined inclusive CC reduced cross sections at $\sqrt{s}=$ $318 \mathrm{GeV}$, as extracted from the combined HERA data, are shown in Fig. 13. The precise predictions describe the CC cross sections well. The $\mathrm{CC}$ data are in general less precise than the $\mathrm{NC}$ data.

The combined inclusive CC reduced cross sections as a function of $x$ in different $Q^{2}$ bins are shown in Fig. 14. The difference between the $e^{-} p$ and the $e^{+} p$ cross sections can

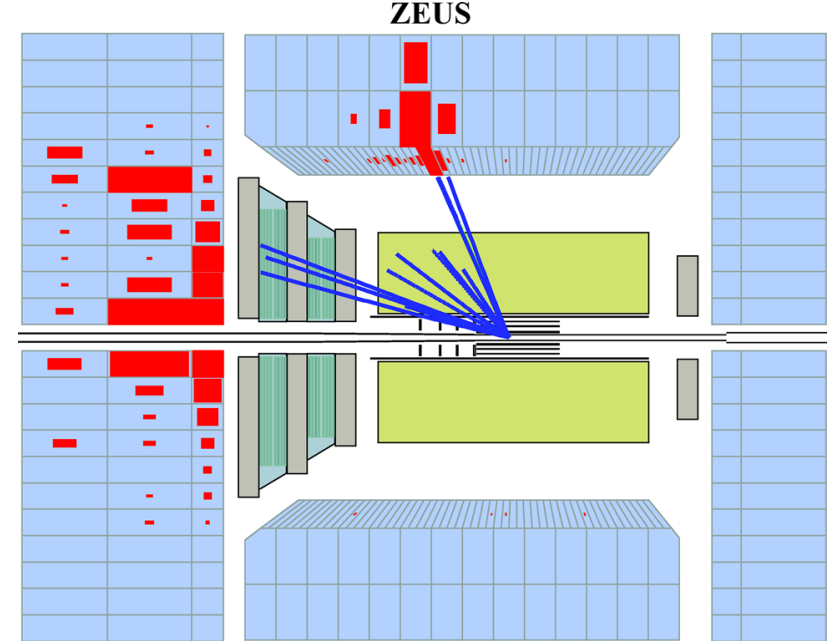

Fig. 12 A charged current event with $Q^{2}=53060 \mathrm{GeV}^{2}$ and $x=0.59$ observed in the ZEUS data. The transverse momentum imbalance in the event is visible in the figure, as most of the activity appears in the upper part of the detector

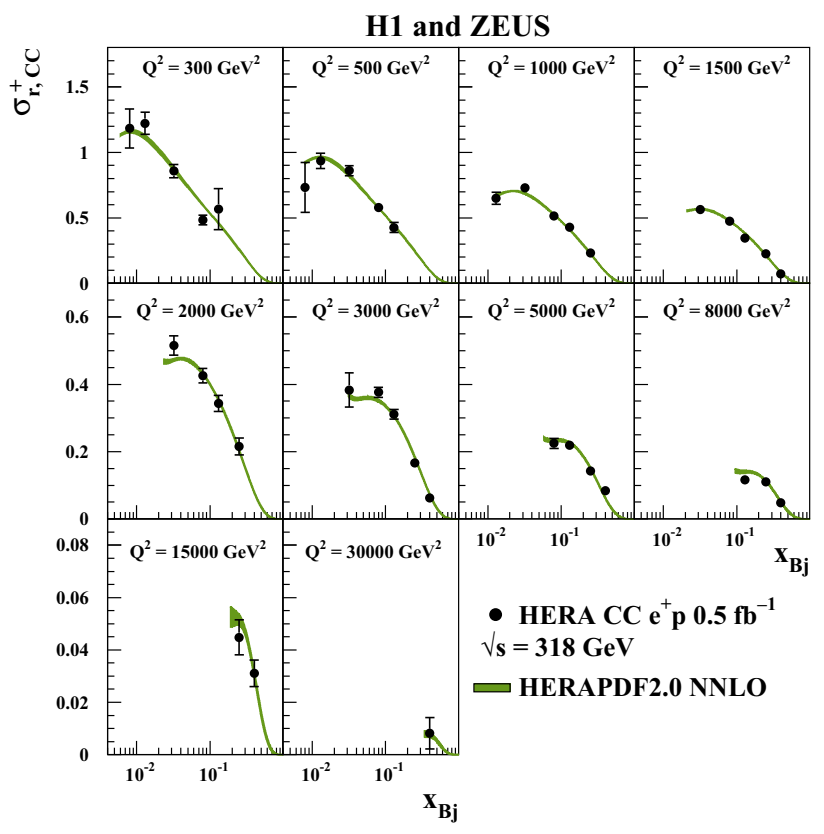

Fig. 13 The combined HERA inclusive $\mathrm{CC} e^{+} p$ reduced cross sections at $\sqrt{s}=318 \mathrm{GeV}$ with overlaid predictions from HERAPDF2.0 NNLO. The bands represent the total uncertainties on the predictions

be intuitively understood at leading order by considering the valence quark composition of the proton and the charge of the exchanged vector boson in $e^{-} p$ and $e^{+} p$ interactions and is explained more formally below. The data are well described by $\mathrm{pQCD}$ predictions.

The cross sections of CC DIS also provide an important input for the determination of the proton structure. In analogy 


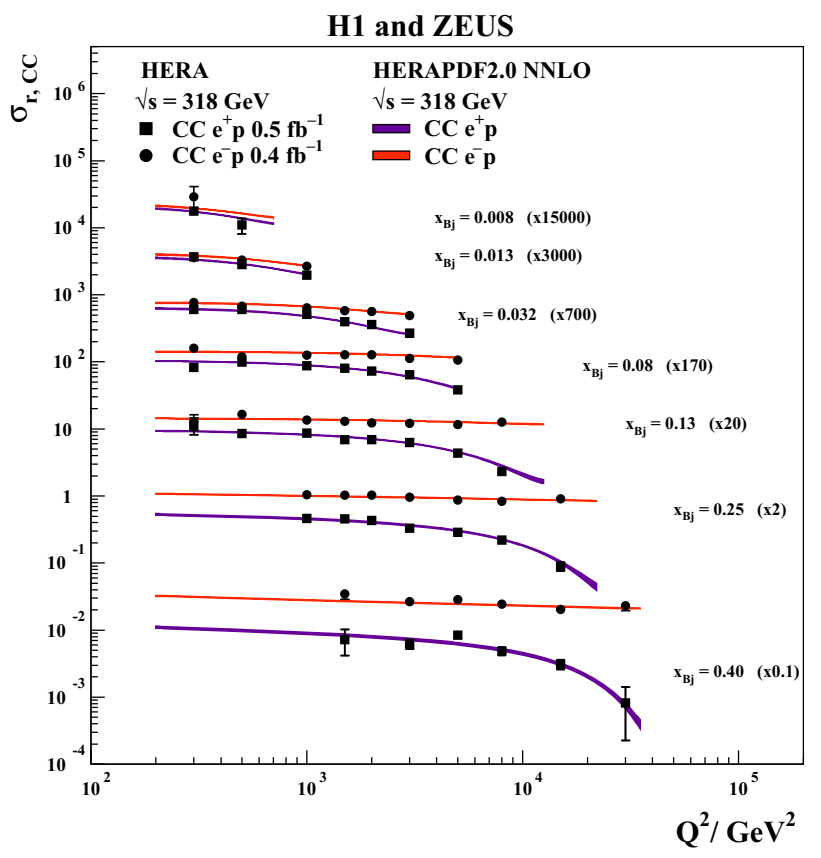

Fig. 14 The combined HERA data for inclusive $\mathrm{CC} e^{+} p$ and $e^{-} p$ reduced cross sections at $\sqrt{s}=318 \mathrm{GeV}$ with overlaid predictions of HERAPDF2.0 NNLO. The bands represent the total uncertainty on the predictions

to Eq. (18), CC structure functions are defined such that

$\sigma_{r, \mathrm{CC}}^{ \pm}=\frac{Y_{+}}{2} W_{2}^{ \pm} \mp \frac{Y_{-}}{2} x W_{3}^{ \pm}-\frac{y^{2}}{2} W_{L}^{ \pm}$

In the QPM [67], $W_{L}^{ \pm}=0$, while $W_{2}^{ \pm}$and $x W_{3}^{ \pm}$are expressed as sum and differences of quark and anti-quark distributions, depending on the charge of the lepton beam:

$W_{2}^{+} \approx x \bar{U}+x D, \quad x W_{3}^{+} \approx x D-x \bar{U}$,

$W_{2}^{-} \approx x U+x \bar{D}, \quad x W_{3}^{-} \approx x U-x \bar{D}$,

and consequently:

$\sigma_{r, \mathrm{CC}}^{+} \approx\left(x \bar{U}+(1-y)^{2} x D\right)$,

$\sigma_{r, \mathrm{CC}}^{-} \approx\left(x U+(1-y)^{2} x \bar{D}\right)$.

The combination of NC and CC DIS measurements therefore makes it possible to determine both the combined sea-quark distributions, $x \bar{U}$ and $x \bar{D}$, and the valence quark distributions, $x u_{v}$ and $x d_{v}$.

Equation (29) also provides the formal explanation for the numerical difference between the cross section for $e^{-} p$ and $e^{+} p$ collisions (see Fig. 14), which is due to the presence of the helicity factor $(1-y)^{2}$. As this factor multiplies the valence quark distribution in the $e^{+} p$ cross section formula, it results in a suppression of the cross section at high $y$. For the $e^{-} p$ cross section, the $(1-y)^{2}$ helicity factor multiplies the anti-quark distribution, which is part of the sea and is

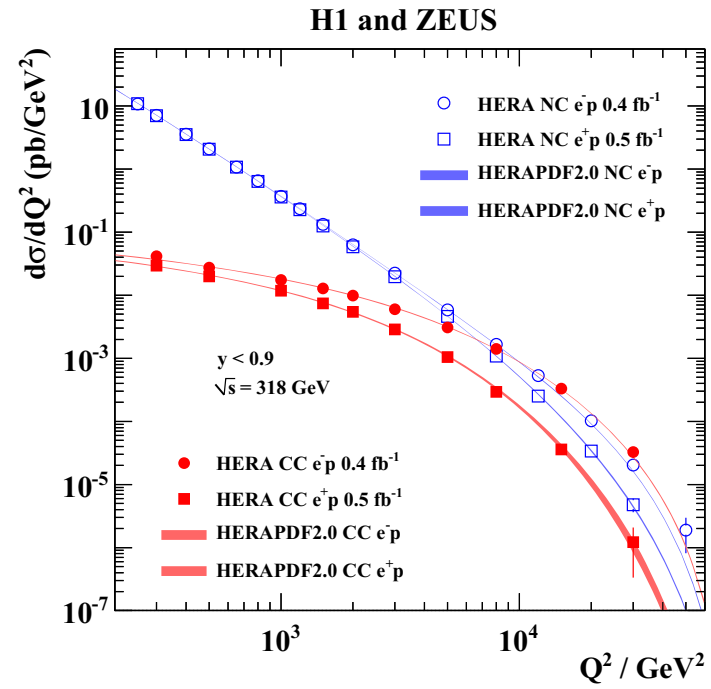

Fig. 15 The combined HERA NC and CC $e^{-} p$ and $e^{+} p$ cross sections together with predictions from HERAPDF2.0 NLO. The bands represent the total uncertainty on the predictions

already suppressed at high $Q^{2}$ and high $x$, and contributes little compared to the quark term.

\subsection{Electroweak unification of NC and CC DIS}

The $\mathrm{H} 1$ and ZEUS combined $\mathrm{NC}$ and $\mathrm{CC}$ cross sections as a function of $Q^{2}$ are shown in Fig. 15. The NC processes dominate the cross section at low $Q^{2}$. This is due to the fact that in the low $Q^{2}$ region the major contribution to the cross section is given by the exchange of a photon between the electron and the proton; $W$ or $Z^{0}$ exchange is suppressed due to the high mass of the vector boson, which enters the propagator term, $Q^{2} /\left(Q^{2}+M_{W / Z}^{2}\right)$. At higher $Q^{2}$, of the order of the mass squared of the $W$ or $Z$ boson, $Q^{2} \simeq 10000 \mathrm{GeV}^{2}, \mathrm{CC}$ and $\mathrm{NC}$ processes are equally important, providing experimental evidence of electroweak unification. It can also be seen that the $e^{-} p$ cross section is higher than the $e^{+} p$ data for both $\mathrm{NC}$ and $\mathrm{CC}$ at higher values of $Q^{2}$ due to the couplings of the quarks in the proton to the exchanged boson. The data are compared with the predictions of HERAPDF2.0 at nextto-leading order (NLO), which provide a good description of the measurements.

\subsection{HERAPDF2.0}

The combined HERA data are used as sole input for the extraction of the PDF set HERAPDF2.0 [81]. The framework already established for the extraction of the HERAPDF1.0 [103] is used. The $x$ and $Q^{2}$ dependences of the NC and CC DIS cross sections were used to determine the free parameters of the assumed shape of the parton distribution functions at a given value of $Q^{2}$. 
As pQCD is not applicable below $Q^{2}$ of the order of the inverse of the proton radius squared, an intrinsic uncertainty is present in performing the QCD fits, due to the choice of the scale at which to start the DGLAP evolution. In the analysis performed to extract the HERAPDF2.0 PDF set, to safely remain in the kinematic region where $\mathrm{pQCD}$ is expected to be applicable, only cross sections for $Q^{2}$ starting from $Q_{\min }^{2}=3.5 \mathrm{GeV}^{2}$ are used in the analysis. The $Q^{2}$ range of the cross sections entering the fit is therefore $3.5 \leq Q^{2} \leq 50000 \mathrm{GeV}^{2}$. The corresponding $x$ range is $0.651 \cdot 10^{-4} \leq x \leq 0.65$. In addition to experimental uncertainties, model and parameterisation uncertainties are also considered.

To extract the proton PDFs, predictions from $\mathrm{pQCD}$ are fitted to the data. These predictions are obtained by solving the DGLAP evolution equations $[10-12,79,80]$ at LO, NLO and NNLO in the $\bar{M} S$ scheme $[104,105]$. The DGLAP equations yield the PDFs at all scales $\mu_{\mathrm{f}}^{2}$ and $x$, if they are provided as functions of $x$ at some starting scale, $\mu_{\mathrm{f}_{0}}^{2}$. This is chosen to be $\mu_{\mathrm{f}_{0}}^{2}=1.9 \mathrm{GeV}^{2}$ as for HERAPDF1.0 [103], since in the used formalism $\mu_{\mathrm{f}_{0}}^{2}$ has to be lower than the charm-quark mass parameter squared. The renormalisation and factorisation scales were chosen to be $\mu_{\mathrm{r}}^{2}=\mu_{\mathrm{f}}^{2}=Q^{2}$.

The values of the charm and beauty mass parameters are chosen after performing $\chi^{2}$ scans of NLO and NNLO pQCD fits to the HERA inclusive data and the $\mathrm{H} 1$ and ZEUS charm and beauty data. The procedure is described in detail elsewhere [106].

A detailed description of the HERAPDF2.0 PDFs is beyond the scope of this paper and further information is given in the publication [81]. Here as an example the quark and gluon distributions are shown in Fig. 16 at a scale $Q^{2}=10 \mathrm{GeV}^{2}$. The gluon distribution dominates the low $x$ region, while at high $x$, as expected, the valence quark distribution are prevalent, and the $u$-type quarks are twice as much as the $d$-type, due to the QED couplings, which are proportional to the quark electrical charge.

HERAPDF2.0 has small experimental uncertainties due to the high precision and coherence of the input data, and makes precise predictions which describe the input data well, as can be seen also in the examples reported above. The precision data on the inclusive $e p$ scattering from the H1 and ZEUS experiments belong to the main legacies of HERA.

\subsection{Helicity structure of the SM and limits on the right-handed $\boldsymbol{W}$ boson}

The presence of a polarised electron beam during the second phase of the data taking period at HERA also provides the opportunity to investigate the helicity structure of the Standard Model. The most striking evidence can be found in CC DIS processes. The electroweak Born-level cross section for

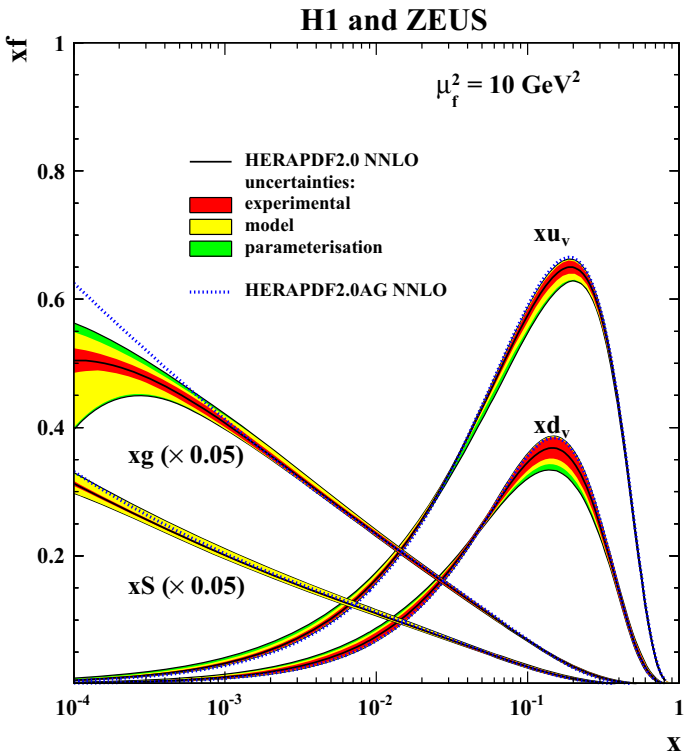

Fig. 16 The parton distribution functions $x u_{v}, x d_{v}, x S=2 x(\bar{U}+\bar{D})$ and $x g$ of HERAPDF2.0 NNLO at $\mu_{\mathrm{f}}^{2}=10 \mathrm{GeV}^{2}$. The gluon and sea distributions are scaled down by a factor 20 . The experimental, model and parameterisation uncertainties are shown. The dotted lines represent HERAPDF2.0AG NNLO with the alternative gluon parameterisation, see [81]

the CC reaction, $e^{+} p \rightarrow \bar{v} X\left(e^{-} p \rightarrow v X\right)$, with a longitudinally polarised positron beam can be expressed as a function of the charged current structure functions $W_{2}^{ \pm}, W_{3}^{ \pm}$ and $W_{L}^{ \pm}[107]$ :

$$
\begin{aligned}
& \frac{\mathrm{d}^{2} \sigma^{C C}\left(e^{ \pm} p\right)}{\mathrm{d} x \mathrm{~d} Q^{2}}=\left(1 \pm \mathcal{P}_{e}\right) \frac{G_{F}^{2}}{2 \pi x}\left(\frac{M_{W}^{2}}{M_{W}^{2}+Q^{2}}\right)^{2} \\
& \times\left[\frac{Y_{+}}{2} W_{2}^{ \pm}-\frac{Y_{-}}{2} x W_{3}^{ \pm}-\frac{y^{2}}{2} W_{L}^{ \pm}\right],
\end{aligned}
$$

where $\mathcal{P}_{e}$ is the polarisation of the lepton beam, as given in Eq. (7).

It can be seen from Eq. (30) that the cross section for the process $e^{-} p \rightarrow v X\left(e^{+} p \rightarrow \bar{v} X\right)$ has a linear dependence on the beam polarisation, and vanishes in the SM for $P_{e^{-}}=1$ $\left(P_{e^{+}}=-1\right)$. The dependence of the CC cross section on the polarisation as measured by the $\mathrm{H} 1$ and ZEUS Collaborations is shown in Fig. 17, compared to an earlier iteration of HERAPDF, namely HERAPDF1.5 [108, 109].The data are well described by the SM predictions.

According to the SM, the CC $e^{+} p$ DIS cross section becomes zero for a fully negatively polarised positron beam, a non-zero cross section at $P_{e}=-1$ might point to the existence of a right-handed $W$ boson, $W_{R}$, and right-handed neutrinos, $v_{R}$. The $\mathrm{H} 1$ and ZEUS data have been used to constrain the mass of such boson [60,110], assuming the coupling strength and propagator dependence on the mass of the boson to be the same as in SM CC interactions, and the outgoing right-handed neutrinos to be light. The ZEUS 


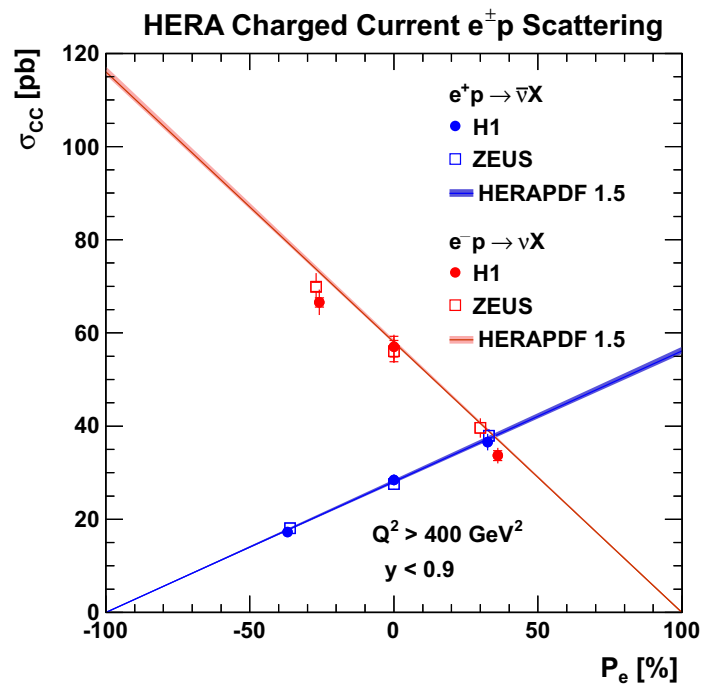

Fig. 17 The measured CC DIS cross sections versus the lepton-beam polarisation as measured by the $\mathrm{H} 1$ and ZEUS Collaborations. The prediction from HERAPDF1.5 is also shown

Collaboration obtains $M_{W_{R}}>198 \mathrm{GeV}$ at $95 \%$ confidence level (CL) [110], while the H1 Collaboration gives a 95 \% CL limit of $M_{W_{R}}>214 \mathrm{GeV}$ and $M_{W_{R}}>194 \mathrm{GeV}$ for $e^{-} p$ and $e^{+} p$ collisions, respectively [60].

The HERA results shown in this section confirm the excellent agreement between the data and the predictions of the SM. However, there are parts of the phase space which are not measured with very high precision, or types of processes for which the cross sections as predicted by the SM are very low and would not manifest themselves in significant changes to the NC and CC inclusive cross sections.

Searches for rare processes and BSM physics at HERA focus therefore on processes with striking features but low SM cross sections, like particle production at high transverse momenta, or specific model-predicted signatures like SUSY, or heavy resonances like leptoquarks, and in general on deviations from SM cross sections in the regions which are less well measured, such as high $Q^{2}$ and high $x$.

\section{Contact interactions}

The high $Q^{2}$ NC DIS interactions $e^{ \pm} p \rightarrow e^{ \pm} X$ at HERA described in the previous section provide the means to search for new physics beyond the SM at short distances, using the concept of four-fermion contact interactions (CI). As opposed to $s$-channel direct searches, where the mass of any resonant particle is limited to the available centre of mass energy via $M_{X}=\sqrt{x s}$, the search for CI relies upon the indirect effects of the interference of the SM photon and $Z$ boson field with the field of any new particle produced. Therefore, CI may manifest as deviations from the SM expectation in the measured differential cross section $\mathrm{d} \sigma / \mathrm{d} Q^{2}$ and any observed deviation may be related to new heavy particles with masses $M_{X}$ much larger than the electroweak scale. In the low-energy limit $\sqrt{s} \ll M_{X}$ such phenomena can be described by an effective four-fermion CI model, and different implementations of such a model are described in the following.

The most general chiral invariant Lagrangian for NC fourfermion CI in ep scattering may be written as [111-114]:

$\mathcal{L}=\sum_{q} \sum_{a, b=L, R}=\eta_{a b}^{q}\left(\bar{e}_{a} \gamma_{\mu} e_{a}\right)\left(\bar{q}_{b} \gamma^{\mu} q_{b}\right)$,

where $\eta_{a b}^{q}$ are the CI coupling coefficients, $a$ and $b$ indicate the left-handed and right-handed fermion helicities and the first sum is over all quark flavours. In the kinematic region of interest at HERA the valence ( $u$ and $d$ ) quarks dominate.

For general models of fermion compositeness or substructure the CI coupling coefficients are defined as

$\eta_{a b}^{q}=\epsilon_{a b}^{q} \frac{4 \pi}{\Lambda^{2}}$

where $\Lambda$ is the compositeness scale and the coefficients $\epsilon_{a b}^{q}$ describe the chiral structure of the coupling and may take the values \pm 1 or 0 , depending on whether the coupling represents pure left-handed (L), right-handed (R), or vector (V) and axial-vector (A) scenarios. Hence, depending on the model and the sign of the coefficients, the new physics process interferes either constructively or destructively with the SM.

Leptoquarks are hypothetical colour triplet scalar or vector bosons carrying both lepton and baryon number, and appear naturally in extensions of the SM which aim to unify the lepton and quark sectors. For leptoquark masses much larger than the probing scale at HERA, $M_{L Q} \gg \sqrt{s}$, the coupling $\lambda$ is related to the CI coupling coefficients via

$\eta_{a b}^{q}=\epsilon_{a b}^{q} \frac{\lambda^{2}}{M_{\mathrm{LQ}}^{2}}$

The classification of leptoquarks is discussed in Sect. 10, and follows the Buchmüller-Rückl-Wyler (BRW) model [115] where the coefficients $\epsilon_{a b}^{q}$ depend on the leptoquark type [116] and take values $0, \pm 0.5, \pm 1$ or \pm 2 .

A proposed explanation as to why the gravitational force is so much weaker than the other fundamental forces, the so-called hierarchy problem, is the existence of large extra dimensions [117-119]. In such a model it is proposed that whilst particles, including strong and electroweak bosons, are confined to four dimensions, gravity can propagate into additional $n$ spatial dimensions, which are compactified with a radius $R$. The theory predicts a new gravitational scale $M_{S}$, related to the Planck scale by $M_{P}^{2} \sim R^{n} M_{S}^{2+n}$, which for $R \sim 1 \mathrm{~mm}$ and $n=2$ can be of order TeV. Consequently, 
at high energies the strengths of the gravitational and electroweak interactions may be comparable [120]. The addition of the graviton-photon interference and graviton- $Z$ interference to the SM cross section is described in [121]. The graviton-exchange contribution to the total $e q \rightarrow e q$ scattering cross section can thus be described $[122,123]$ as a contact interaction with an effective coupling strength:

$\eta_{G}=\frac{\lambda}{M_{S}^{4}}$

where the coupling $\lambda$ is conventionally set to \pm 1 .

Finally, the observation of finite size effects such as the measurement of the electroweak charge distribution of fermions would provide a clear manifestation of quark substructure. Such effects are typically described by a standard form factor in the $e q \rightarrow e q$ scattering cross section:

$f\left(Q^{2}\right)=1-\frac{\left\langle R^{2}\right\rangle}{6} Q^{2}$

where $\left\langle R^{2}\right\rangle$ is the mean squared radius of the electroweak charge distribution of the quark, or quark radius. This form factor modifies the $Q^{2}$ dependence of the SM cross section similarly to the above CI models.

Several results on searches for contact interactions have been published by both $\mathrm{H} 1$ and ZEUS using partial HERA data sets $[120,121,124,125]$. In this review we focus on the H1 result [126] that uses their full data sample, corresponding to an integrated luminosity of $446 \mathrm{pb}^{-1}$ as described in Table 1. As some of the models described above exhibit chiral sensitivity, the longitudinally polarised data sets are analysed separately.

To investigate contact interactions the measured $\mathrm{H} 1 \mathrm{NC}$ differential cross sections $\mathrm{d} \sigma / \mathrm{d} Q^{2}$ at high four-momentum transfer squared $Q^{2}>200 \mathrm{GeV}^{2}$ are compared to the $\mathrm{SM}$ prediction. The standard $\mathrm{H} 1 \mathrm{NC}$ event selection is employed [60]. For the analysis of contact interactions, the SM prediction at high $Q^{2}$ and high $x$ are of special relevance. The use of a PDF set to determine the SM prediction in this kinematic region based mostly on the HERA data is not appropriate, as the SM predictions would also include the effects from any potential contact interactions present in the data. Therefore, the PDF set CTEQ6m PDF [127] is used. The CTEQ6m set was obtained by fitting several experimental data sets. At high $x$ this PDF is mostly constrained by fixed-target experiments and also by $W$-boson production and jet data from the Tevatron experiments, which are not sensitive to possible $e q$ contact interaction processes. CTEQ6m does include early $e^{ \pm} p$ scattering data at high $Q^{2}$ from the $\mathrm{H} 1$ and ZEUS experiments, but as the $e^{+} p\left(e^{-} p\right)$ data sets analysed here are 6(10) times larger, the residual correlations between the HERA data and the CTEQ6m PDF are small and are neglected in the following. The CTEQ6m parton densi- ties can be therefore be regarded as unbiased with respect to possible contact interaction effects. CTEQ6m is chosen as it describes many experimental data and in particular, the HERA data in the region $Q^{2}<200 \mathrm{GeV}^{2}$, which are not used in this analysis. The analysis was also verified using an alternative PDF not based on HERA high $Q^{2}$ data, a dedicated H1 PDF set, obtained from a next-to-leading order QCD fit to the H1 data [82] with $Q^{2}<200 \mathrm{GeV}^{2}$, excluding the high $Q^{2}$ data used in this analysis. Both the SM expectation and limits derived using the dedicated H1 PDF agree well with those obtained using the CTEQ6m PDF within the uncertainties. The ratio of the measured cross sections to those from the SM prediction is shown in Fig. 18, where good agreement is observed both in the full data set and each of the polarised data sets from the HERA II.

A quantitative test is performed to evaluate the impact of each of the different CI models on the SM prediction and the level of agreement to the data using a $\chi^{2}$ minimisation fitting function [125]. The fit to the experimental and theoretical cross sections fully takes into account all statistical and systematic uncertainties.

The H1 data are found to be consistent with the SM expectation based on the CTEQ6m PDF, yielding a $\chi^{2} /$ dof $=$ $16.4 / 17(7.0 / 17)$ for the $e^{+} p\left(e^{-} p\right)$ data, where 17 is the number of $Q^{2}$ bins. For each of the CI models described above, the effective scale parameters and couplings associated to the new physics scale are determined by a fit to the differential NC cross section. All scale parameters are found to be consistent with the SM and limits are calculated at the $95 \%$ CL using the frequentist method as described in the H1 HERA I publication [125].

Lower limits on the compositeness scale $\Lambda$ in the context of the general CI model are presented in Table 2. The results are presented for nine scenarios, which differ in their chiral structure as determined by the CI coupling coefficients $\eta_{a b}^{q}$. Depending on the model and the sign of the coefficients, limits on $\Lambda$ are obtained in the range $3.6 \mathrm{TeV}\left(\Lambda_{V A}^{-}\right)$to $7.2 \mathrm{TeV}\left(\Lambda_{V V}^{-}\right)$. In a similar analysis by ZEUS [120] using their HERA I data alone, limits of 3.3 and $6.2 \mathrm{TeV}$ are found for $\Lambda_{V A}^{-}$and $\Lambda_{V V}^{-}$, respectively. For comparison, in a recent ATLAS analysis where the background process to contact interactions arises from Drell-Yan production $q q \rightarrow \ell \ell$, limits are derived at the $95 \% \mathrm{CL}$ on the LL, LR and RR scenarios in the range 15.4 and $26.3 \mathrm{TeV}$ [128].

Within the model of leptoquark-type contact interactions lower limits on $M_{L Q} / \lambda$ at the $95 \% \mathrm{CL}$ are presented in Table 3, for each of the 14 leptoquarks in the BRW model. The observed limits in this analysis are in the range $M_{L Q} / \lambda>$ $0.41-1.86 \mathrm{TeV}$. Leptoquarks coupling to $u$ quarks are probed with higher sensitivity, corresponding to more stringent limits than those coupling to $d$ quarks due to the valence quark content of the proton and the QED couplings of the electron to the quarks, which reflect the electric charge of the different 
Fig. 18 The ratio of the measured cross section to the SM prediction determined using the CTEQ6m PDF set for $e^{+} p \rightarrow e X$ and $e^{-} p \rightarrow e X$ scattering. Figure a shows the full $\mathrm{H} 1$ data with an average longitudinal polarisation of $\mathcal{P}_{e} \sim 0$. Figures $\mathbf{b}$ and $\mathbf{c}$ show the polarised $\mathrm{H} 1$ data divided into the different lepton charge and polarisation data sets. The error bars represent the statistical and uncorrelated systematic errors added in quadrature. The bands indicate the PDF uncertainties of the SM cross section predictions
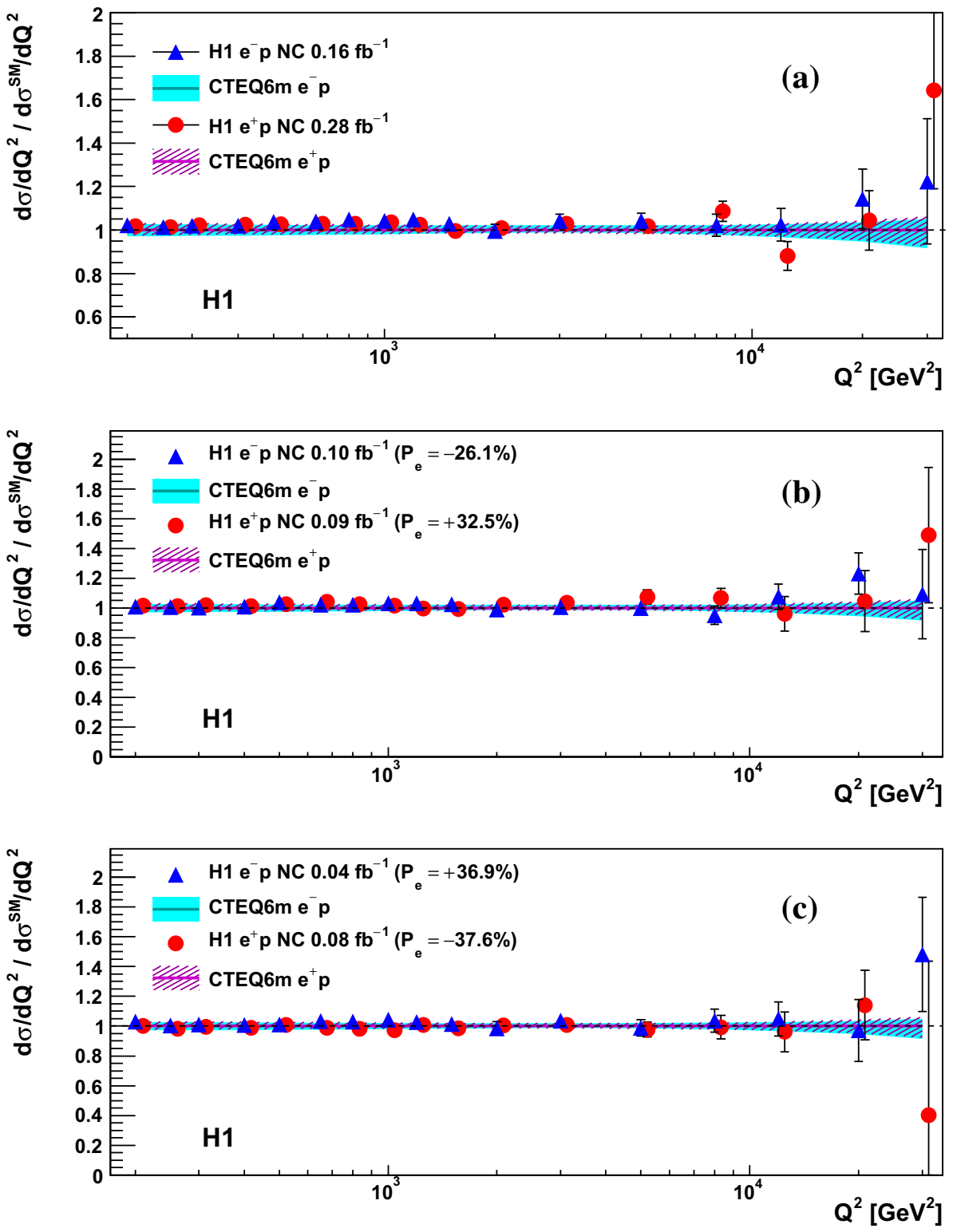

Table 2 Lower limits from $\mathrm{H} 1$ at the $95 \% \mathrm{CL}$ on the compositeness scale $\Lambda$. The $\Lambda^{+}$ limits correspond to the upper signs and the $\Lambda^{-}$limits correspond to the lower signs of the chiral coefficients $\epsilon_{L L}^{q}, \epsilon_{L R}^{q}$, $\epsilon_{R L}^{q}$ and $\epsilon_{R R}^{q}$, where the CI coupling coefficient is given by $\eta_{a b}^{q}=\epsilon_{a b}^{q} 4 \pi / \Lambda^{2}$

\begin{tabular}{lllllll}
\hline \multicolumn{7}{l}{ H1 search for general compositeness $\left(\mathcal{L}=446 \mathrm{pb}^{-1}\right)$} \\
\hline Model & $\epsilon_{L L}^{q}$ & $\epsilon_{L R}^{q}$ & $\epsilon_{R L}^{q}$ & $\epsilon_{R R}^{q}$ & $\Lambda^{+}(\mathrm{TeV})$ & $\Lambda^{-}(\mathrm{TeV})$ \\
\hline LL & \pm 1 & 0 & 0 & 0 & 4.2 & 4.0 \\
LR & 0 & \pm 1 & 0 & 0 & 4.8 & 3.7 \\
RL & 0 & 0 & \pm 1 & 0 & 4.8 & 3.8 \\
RR & 0 & 0 & 0 & \pm 1 & 4.4 & 3.9 \\
VV & \pm 1 & \pm 1 & \pm 1 & \pm 1 & 5.6 & 7.2 \\
AA & \pm 1 & $\mp 1$ & $\mp 1$ & \pm 1 & 4.4 & 5.1 \\
VA & \pm 1 & $\mp 1$ & \pm 1 & $\mp 1$ & 3.8 & 3.6 \\
LL+RR & \pm 1 & 0 & 0 & \pm 1 & 5.3 & 5.1 \\
LR+RL & 0 & \pm 1 & \pm 1 & 0 & 5.4 & 4.8 \\
\hline
\end{tabular}


Table 3 Lower limits from $\mathrm{H} 1$ at the $95 \% \mathrm{CL}$ on $M_{L Q} / \lambda$ for 14 scalar (S) and vector (V) leptoquarks, where $\mathrm{L}$ and $\mathrm{R}$ denote the lepton chirality and the subscript $(0,1 / 2,1)$ is the weak isospin. In this case the CI coupling coefficient is $\eta_{a b}^{q}=\epsilon_{a b}^{q} \lambda^{2} / M_{L Q}^{2}$. For each leptoquark type, the relevant coefficients $\epsilon_{a b}^{q}$ and fermion number $F=L+3 B$ are indicated. Leptoquarks with identical quantum numbers except for weak hypercharge are distinguished using a tilde, for example $V_{0}^{R}$ and $\tilde{V}_{0}^{R}$

H1 search for heavy leptoquarks $\left(\mathcal{L}=446 \mathrm{pb}^{-1}\right)$

\begin{tabular}{lllll}
\hline $\mathrm{LQ}$ & $\epsilon_{a b}^{u}$ & $\epsilon_{a b}^{d}$ & $F$ & $M_{L Q} / \lambda(\mathrm{TeV})$ \\
\hline$S_{0}^{L}$ & $\epsilon_{L L}^{u}=+\frac{1}{2}$ & & 2 & 1.10 \\
$S_{0}^{R}$ & $\epsilon_{R R}^{u}=+\frac{1}{2}$ & & 2 & 1.10 \\
$\tilde{S}_{0}^{R}$ & & $\epsilon_{R R}^{d}=+\frac{1}{2}$ & 2 & 0.41 \\
$S_{1 / 2}^{L}$ & $\epsilon_{L R}^{u}=-\frac{1}{2}$ & & 0 & 0.87 \\
$S_{1 / 2}^{R}$ & $\epsilon_{R L}^{u}=-\frac{1}{2}$ & $\epsilon_{R L}^{d}=-\frac{1}{2}$ & 0 & 0.59 \\
$\tilde{S}_{1 / 2}^{L}$ & & $\epsilon_{L R}^{d}=-\frac{1}{2}$ & 0 & 0.66 \\
$S_{1}^{L}$ & $\epsilon_{L L}^{u}=+\frac{1}{2}$ & $\epsilon_{L L}^{d}=+1$ & 2 & 0.71 \\
$V_{0}^{L}$ & & $\epsilon_{L L}^{d}=-1$ & 0 & 1.06 \\
$V_{0}^{R}$ & & $\epsilon_{R R}^{d}=-1$ & 0 & 0.91 \\
$\tilde{V}_{0}^{R}$ & $\epsilon_{R R}^{u}=-1$ & & 0 & 1.35 \\
$V_{1 / 2}^{L}$ & & $\epsilon_{L R}^{d}=+1$ & 2 & 0.51 \\
$V_{1 / 2}^{R}$ & $\epsilon_{R L}^{u}=+1$ & $\epsilon_{R L}^{d}=+1$ & 2 & 1.44 \\
$\tilde{V}_{1 / 2}^{L}$ & $\epsilon_{L R}^{u}=+1$ & & 2 & 1.58 \\
$V_{1}^{L}$ & $\epsilon_{L L}^{u}=-2$ & $\epsilon_{L L}^{d}=-1$ & 0 & 1.86 \\
\hline
\end{tabular}

quarks. For a Yukawa coupling of electromagnetic strength, $\lambda=0.3$, scalar and vector leptoquark masses up to 0.33 and $0.56 \mathrm{TeV}$ are excluded, respectively.

The $S_{0}^{L}$ and $\tilde{S}_{1 / 2}^{L}$ leptoquarks have identical quantum numbers to down and up squarks, respectively. The couplings associated to these leptoquarks therefore correspond to the Yukawa couplings $\lambda_{11 k}^{\prime}$ and $\lambda_{1 j 1}^{\prime}$ in the framework of $R_{p}$ violating supersymmetry and the limits on these leptoquarks presented in Table 3 are also applicable to the ratio $M_{\tilde{q}} / \lambda^{\prime}$ [126]. Dedicated leptoquark searches performed at HERA are discussed in the following sections.

Lower limits in a model with large extra dimensions on the gravitational scale $M_{S}$ in $4+n$ dimensions are derived assuming a positive or negative coupling. For a $\lambda=+1(\lambda=$ -1) coupling mass scales $M_{S}<0.90 \mathrm{TeV}\left(M_{S}<0.92 \mathrm{TeV}\right)$ are excluded at the $95 \% \mathrm{CL}$. The corresponding cross section predictions normalised to the SM expectation are compared to the $e^{ \pm} p$ data in Fig. 19. Since the advent of the LHC and the higher energy centre of mass data that it provides these limits have been surpassed, for example by ATLAS, where limits are set on $M_{S}>3.2 \mathrm{TeV}$ [128].

Finally, an upper limit at $95 \% \mathrm{CL}$ on the quark radius $R_{q}<0.65 \cdot 10^{-18} \mathrm{~m}$ is found, assuming point-like leptons. The same limit from ZEUS is $R_{q}<0.81 \cdot 10^{-18} \mathrm{~m}$, which is derived in a similar analysis using their HERA I data set [120].

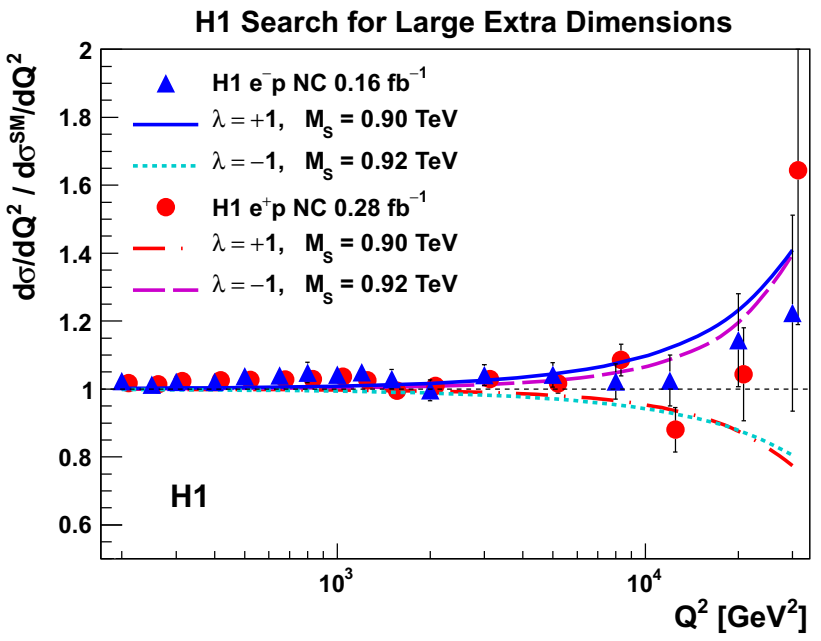

Fig. 19 The measured $\mathrm{NC}$ cross section $\mathrm{d} \sigma / \mathrm{d} Q^{2}$ normalised to the SM expectation. $\mathrm{H} 1 e^{ \pm} p$ scattering data are compared with curves corresponding to $95 \% \mathrm{CL}$ exclusion limits obtained from the full $\mathrm{H} 1$ data on the gravitational scale, $M_{S}$, for both positive $(\lambda=+1)$ and negative $(\lambda=-1)$ couplings. The error bars represent the statistical and uncorrelated systematic errors added in quadrature

\section{Leptoquarks}

The electron-proton collisions at HERA provide a unique opportunity to search for new particles coupling directly to a lepton and a quark. Leptoquarks (LQs) are an example of such particles, colour-triplet bosons which appear in many extensions of the SM [129-139]. They can be produced at HERA directly via $s$-channel resonant production, or indirectly via $u$-channel virtual LQ exchange, as shown in Fig. 20. The coupling at the electron-quark-LQ vertex is described by a dimensionless parameter $\lambda$. LQs carry both lepton $(L)$ and baryon $(B)$ quantum numbers and the fermion number $F=L+3 B$ is conserved. The $s$-channel production dominates for LQ masses lower than the centre of mass energy, while for masses larger than $\sqrt{s}$ both the $s$ - and the $u$ channels, as well as the interference with SM processes, are important. In the first case, LQs appear as peaks in the spectra of the final-state lepton-jet invariant mass. In the second case, heavy LQ exchange could lead to measurable low-energy exchange and so to deviations of the measured cross sections
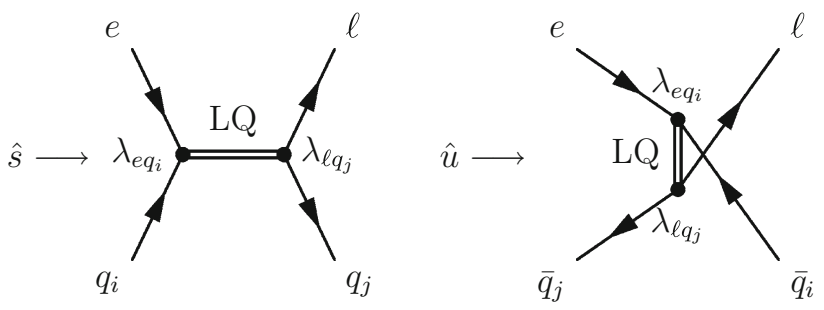

Fig. 20 Leptoquark production in ep collisions: $s$-channel resonant production (left) and $u$-channel virtual exchange (right) with subsequent decay to a lepton-quark pair 
Table 4 The 14 LQ types of the Buchmüller-Rückl-Wyler classification in the commonly used notation. The LQ subscripts refer to the weak isospin and the superscripts refer to the lepton chirality. The spin $J$, fermion number $F$ and charge $Q$ of each leptoquark is indicated, as well as the dominant resonant production process in $e p$ scattering and possible decay modes, the corresponding coupling and the branching ratio to charged leptons $\beta_{\ell}$

\begin{tabular}{|c|c|c|c|c|c|c|c|c|}
\hline \multirow{3}{*}{$\begin{array}{c}\text { LQ type } \\
S_{0}^{L}\end{array}$} & \multirow{3}{*}{$\begin{array}{l}J \\
0\end{array}$} & \multirow{3}{*}{$\begin{array}{l}F \\
2\end{array}$} & \multirow{3}{*}{$\begin{array}{c}Q \\
-1 / 3\end{array}$} & \multicolumn{3}{|c|}{$\begin{array}{c}\text { Production and } \\
\text { decay modes }\end{array}$} & \multirow{3}{*}{$\begin{array}{c}\text { Coupling } \\
\lambda_{L} \\
-\lambda_{L}\end{array}$} & \multirow{3}{*}{$\begin{array}{l}\beta_{\ell} \\
1 / 2 \\
1 / 2\end{array}$} \\
\hline & & & & & & $\overline{\ell^{-} u}$ & & \\
\hline & & & & $e_{L} u_{L}$ & $\rightarrow$ & $\nu_{\ell} d$ & & \\
\hline$S_{0}^{R}$ & 0 & 2 & $-1 / 3$ & $e_{R}^{-} u_{R}$ & $\rightarrow$ & $\ell^{-} u$ & $\lambda_{R}$ & 1 \\
\hline$\tilde{S}_{0}^{R}$ & 0 & 2 & $-4 / 3$ & $e_{R}^{-} d_{R}$ & $\rightarrow$ & $\ell^{-} d$ & $\lambda_{R}$ & 1 \\
\hline \multirow{3}{*}{$S_{1}^{L}$} & \multirow{3}{*}{0} & \multirow{3}{*}{2} & \multirow{2}{*}{$-1 / 3$} & \multirow{2}{*}{$e_{L}^{-} u_{L}$} & & $\ell^{-} u$ & $-\lambda_{L}$ & $1 / 2$ \\
\hline & & & & & & $\nu_{\ell} d$ & $-\lambda_{L}$ & $1 / 2$ \\
\hline & & & $-4 / 3$ & $e_{L}^{-} d_{L}$ & $\rightarrow$ & $\ell^{-} d$ & $-\sqrt{2} \lambda_{L}$ & 1 \\
\hline$V_{1 / 2}^{L}$ & 1 & 2 & $-4 / 3$ & $e_{L}^{-} d_{R}$ & $\rightarrow$ & $\ell^{-} d$ & $\lambda_{L}$ & 1 \\
\hline \multirow{2}{*}{$V_{1 / 2}^{R}$} & \multirow{2}{*}{1} & \multirow{2}{*}{2} & $-1 / 3$ & $e_{R}^{-} u_{L}$ & $\rightarrow$ & $\ell^{-} u$ & $\lambda_{R}$ & 1 \\
\hline & & & $-4 / 3$ & $e_{R}^{-} d_{L}$ & $\rightarrow$ & $\ell^{-} d$ & $\lambda_{R}$ & 1 \\
\hline$\tilde{V}_{1 / 2}^{L}$ & 1 & 2 & $-1 / 3$ & $e_{L}^{-} u_{R}$ & $\rightarrow$ & $\ell^{-} u$ & $\lambda_{L}$ & 1 \\
\hline \multirow{2}{*}{$V_{0}^{L}$} & \multirow{2}{*}{1} & \multirow{2}{*}{0} & \multirow{2}{*}{$+2 / 3$} & \multirow{2}{*}{$e_{R}^{+} d_{L}$} & & $\ell^{+} d$ & $\lambda_{L}$ & $1 / 2$ \\
\hline & & & & & & $\bar{\nu}_{\ell} u$ & $\lambda_{L}$ & $1 / 2$ \\
\hline$V_{0}^{R}$ & 1 & 0 & $+2 / 3$ & $e_{L}^{+} d_{R}$ & $\rightarrow$ & $\ell^{+} d$ & $\lambda_{R}$ & 1 \\
\hline$\tilde{V}_{0}^{R}$ & 1 & 0 & $+5 / 3$ & $e_{L}^{+} u_{R}$ & $\rightarrow$ & $\ell^{+} u$ & $\lambda_{R}$ & 1 \\
\hline \multirow{3}{*}{$V_{1}^{L}$} & \multirow{3}{*}{1} & \multirow{3}{*}{0} & \multirow{2}{*}{$+2 / 3$} & \multirow{2}{*}{$e_{R}^{+} d_{L}$} & & $\ell^{+} d$ & $-\lambda_{L}$ & $1 / 2$ \\
\hline & & & & & & $\bar{\nu}_{\ell} u$ & $\lambda_{L}$ & $1 / 2$ \\
\hline & & & $+5 / 3$ & $e_{R}^{+} u_{L}$ & $\rightarrow$ & $\ell^{+} u$ & $\sqrt{2} \lambda_{L}$ & 1 \\
\hline$S_{1 / 2}^{L}$ & 0 & 0 & $+5 / 3$ & $e_{R}^{+} u_{R}$ & $\rightarrow$ & $\ell^{+} u$ & $\lambda_{L}$ & 1 \\
\hline \multirow{2}{*}{$S_{1 / 2}^{R}$} & \multirow{2}{*}{0} & \multirow{2}{*}{0} & $+2 / 3$ & $e_{L}^{+} d_{L}$ & $\rightarrow$ & $\ell^{+} d$ & $-\lambda_{R}$ & 1 \\
\hline & & & $+5 / 3$ & $e_{L}^{+} u_{L}$ & $\rightarrow$ & $\ell^{+} u$ & $\lambda_{R}$ & 1 \\
\hline$\tilde{S}_{1 / 2}^{L}$ & 0 & 0 & $+2 / 3$ & $e_{R}^{+} d_{R}$ & $\rightarrow$ & $\ell^{+} d$ & $\lambda_{L}$ & 1 \\
\hline
\end{tabular}

with respect to SM predictions. For LQ masses lower than $\sqrt{s}, F=0(F=2)$ LQs dominate in $e^{+} p\left(e^{-} p\right)$ collisions, while for higher masses both $e^{+} p$ and $e^{-} p$ have similar sensitivity to all LQ types.

In the framework of the phenomenological BuchmüllerRückl-Wyler (BRW) model [115], LQs are classified into 14 types [116] with respect to the quantum numbers spin $J$, weak isospin $I$ and chirality $C$ (left-handed $L$, right-handed, $R)$. The 14 different LQs are detailed in Table 4. Scalar $(J=$ $0)$ LQs are denoted $S_{I}^{C}$ and vector $(J=1)$ LQs are denoted $V_{I}^{C}$ in the following. LQs with identical quantum numbers except for weak hypercharge are distinguished using a tilde, for example $V_{0}^{R}$ and $\tilde{V}_{0}^{R}$.

Whereas all 14 LQs couple to electron-quark pairs, four of the left-handed LQs, namely $S_{0}^{L}, S_{1}^{L}, V_{0}^{L}$ and $V_{1}^{L}$, may also decay to a neutrino-quark pair. The branching ratio to charged leptons is given by

$\beta_{\ell}=\Gamma_{\ell q} /\left(\Gamma_{\ell q}+\Gamma_{v_{\ell} q}\right)$ where $\Gamma_{\ell q}\left(\Gamma_{v_{\ell} q}\right)$ denotes the partial width for the LQ decay to the charged lepton $\ell$ (neutrino $v_{\ell}$ ) and a quark $q$. The branching fraction of decays into a neutrino-quark pair is then given by $\beta_{\nu_{\ell}}=1-\beta_{\ell}$. In particular, for $S_{0}^{L}$ and $V_{0}^{L}$ the branching fraction of decays into an electron-quark pair is predicted $^{7}$ by the model to be $\beta_{\ell}=0.5$.

If the lepton number is conserved, a LQ can decay only into a quark and a first generation lepton: an electron, positron or electron-neutrino, and is termed a first generation leptoquark. If the lepton number is not conserved, the production of second and third generation leptoquarks is possible, leading to a final state containing a second or third generation lepton together with the quark.

The BRW model assumes lepton number conservation, although a general extension of this model allows for the decay of LQs to final states containing a quark and a lepton of a different flavour, that is, a muon or tau lepton, introduc-

\footnotetext{
$\overline{7 \text { In the case of }} S_{1}^{L}$ and $V_{1}^{L}$ LQs, which are superpositions of two states, this branching ratio is less trivial.
} 
ing lepton flavour violation (LFV). Non-zero couplings $\lambda_{e q_{i}}$ to an electron-quark pair and $\lambda_{\mu q_{j}}\left(\lambda_{\tau q_{j}}\right)$ to a muon(tau)quark pair are assumed. The indices $i$ and $j$ represent quark generation indices, such that $\lambda_{e q_{i}}$ denotes the coupling of an electron to a quark of generation $i$, and $\lambda_{\ell q_{j}}$ is the coupling of the outgoing lepton (where $\ell=\mu$ or $\tau$ ) to a quark of generation $j$. When second and third generation LQs are considered, the branching ratio $\beta$ to a given charged lepton flavour $L Q \rightarrow \mu(\tau) q$ is calculated as

$\beta=\beta_{\ell} \times \beta_{L F V}$

where

$\beta_{L F V}=\frac{\Gamma_{\mu(\tau) q}}{\Gamma_{\mu(\tau) q}+\Gamma_{e q}}$

and

$\Gamma_{\ell q}=m_{\mathrm{LQ}} \lambda_{\ell q}^{2} \times \begin{cases}\frac{1}{16 \pi} & \text { scalar LQ } \\ \frac{1}{24 \pi} & \text { vector LQ }\end{cases}$

where $\Gamma_{\ell q}$ denotes the partial LQ decay width for the decay to a lepton $\ell=e, \mu, \tau$ and a quark $q$. Assuming lepton universality, and that only one LFV transition is possible, $\beta_{L F V}=0.5$. An overview of this extended model for the LQ coupling to $u$ and $d$ quarks is provided elsewhere [140].

\subsection{Searches for first generation leptoquarks}

Searches for first generation leptoquarks have been regularly performed by both $\mathrm{H} 1$ and ZEUS during the HERA data taking [141-145], and two papers published in 1997 using up to $20 \mathrm{pb}^{-1}$ of the first data taken revealed a handful of outstanding events observed by both collaborations at masses above and around $200 \mathrm{GeV}[146,147]$.

The final $\mathrm{H} 1$ and ZEUS first generation leptoquark searches are summarised in this section, which between them include the full $1 \mathrm{fb}^{-1}$ of HERA data [148, 149]. The polarisation $\mathcal{P}_{e}$ of the lepton beam in the HERA II data taking period is exploited to enhance the chiral sensitivity of leptoquarks by analysing the positively and negatively polarised data samples separately.

First generation leptoquarks lead to final states which are similar to those of NC and CC DIS, in that the LQ decays into either an electron or neutrino and a quark. As the experimental signature of $\mathrm{LQ}$ production is similar to that of $\mathrm{NC}$ and $\mathrm{CC}$ events, the data selection closely follows that used in the standard inclusive DIS analyses performed by $\mathrm{H} 1$ and ZEUS as described in Sect. 8. The NC and CC DIS processes are modelled using the DJANGOH generator and the smaller photoproduction contribution is estimated using PYTHIA.

In the NC-like event topology $e p \rightarrow e X$, the $\mathrm{H} 1$ analysis [148] is performed in the region $Q^{2}>133 \mathrm{GeV}^{2}$,
$E_{e^{\prime}}>11 \mathrm{GeV}$ and $0.1<y<0.9$, where the electron reconstruction method is used (see Eq. (9a)). The ZEUS analysis [149] employs both the electron and double angle methods (see Eq. (15a)) and the phase space is defined as $Q^{2}>2500 \mathrm{GeV}^{2}, E_{e^{\prime}}>10 \mathrm{GeV}, x>0.1$ and $y_{e}<0.95$. An identified hadronic jet with $P_{T}>15 \mathrm{GeV}$ in the region $|\eta|<3$ is also required by the ZEUS analysis. Background from neutral hadrons or photons misidentified as leptons is suppressed by requiring a charged track to be associated to the lepton candidate and both analyses use additional cuts on the total $E-P_{z}$ in the event to reduce the residual photoproduction background.

In the CC-like event topology ep $\rightarrow v X$, events are selected in the $\mathrm{H} 1$ analysis by requiring significant missing transverse momentum $P_{T}^{\text {miss }}>12 \mathrm{GeV}$, which is due to the undetected neutrino, in the inelasticity region $0.1<y<$ 0.85 . The ZEUS analysis phase space is $P_{T}^{\text {miss }}>22 \mathrm{GeV}$, $Q^{2}>700 \mathrm{GeV}^{2}, y<0.9$. An identified hadronic jet with $P_{T}>10 \mathrm{GeV}$ in the region $|\eta|<3$ is also required. Photoproduction background is suppressed in both the $\mathrm{H} 1$ and ZEUS analyses by exploiting the correlation between $P_{T}^{\text {miss }}$ and the ratio $V_{\mathrm{ap}} / V_{\mathrm{p}}$ [102]. Both $\mathrm{H} 1$ and ZEUS use the hadronic reconstruction method described in Eq. (10) for the CC-like event topology.

The lepton scattering angle in the lepton-jets centre of mass frame, $\theta^{*}$, is used in the ZEUS analysis to isolate a potential leptoquark signal further. The decay of a scalar resonance results in a flat distribution in $\cos \theta^{*}$, while SM events show an approximately $1 /\left(1-\cos \theta^{*}\right)^{2}$ dependence [143]. In a final step a cut of $\cos \theta^{*}<0.4$ is applied by ZEUS in their analysis of both the $\mathrm{NC}$ and $\mathrm{CC}$ topologies.

The leptoquark mass is reconstructed by $\mathrm{H} 1$ as

$M_{\mathrm{LQ}}=\sqrt{Q^{2} / y}$

and uses the measured kinematics of the scattered electron (hadronic final state) in the analysis of $\mathrm{NC}(\mathrm{CC})$ topologies. In the ZEUS analysis, the LQ mass is reconstructed from the momentum and energy of the jet and the lepton:

$M_{l j s}=\sqrt{E_{l j s}^{2}-\mathbf{p}_{l j s}^{2}}$,

where $E_{l j s}^{2}$ is the sum of the energies of the jet and the lepton, and $\mathbf{p}_{l j s}^{2}$ is the vector sum of their momenta.

In the analysis of their complete data sets good agreement between the data and the SM is observed by both $\mathrm{H} 1$ and ZEUS and no evidence for LQs at HERA is observed. The SM expectation is dominated by DIS processes in all event samples, with small additional contributions from photoproduction.

Mass spectra of the four H1 HERA II data sets taken with a longitudinally polarised lepton beam are shown in Fig. 21, 
Fig. 21 The reconstructed leptoquark mass in the search for first generation leptoquarks in the 2003-2007 H1 data, which was taken with a polarised lepton beam. The left-handed electron data (a) and left-handed positron data (b) are shown in the top row; the right-handed electron data (c) and right-handed positron data (d) are shown in the bottom row. The luminosity $\mathcal{L}$ and average longitudinal lepton polarisation $\mathcal{P}_{e}$ of each data set is indicated. The NC (solid points) and CC (open points) data are compared to the SM predictions

(histograms), where the shaded bands indicate the total SM uncertainties
H1 Search for First Generation Leptoquarks
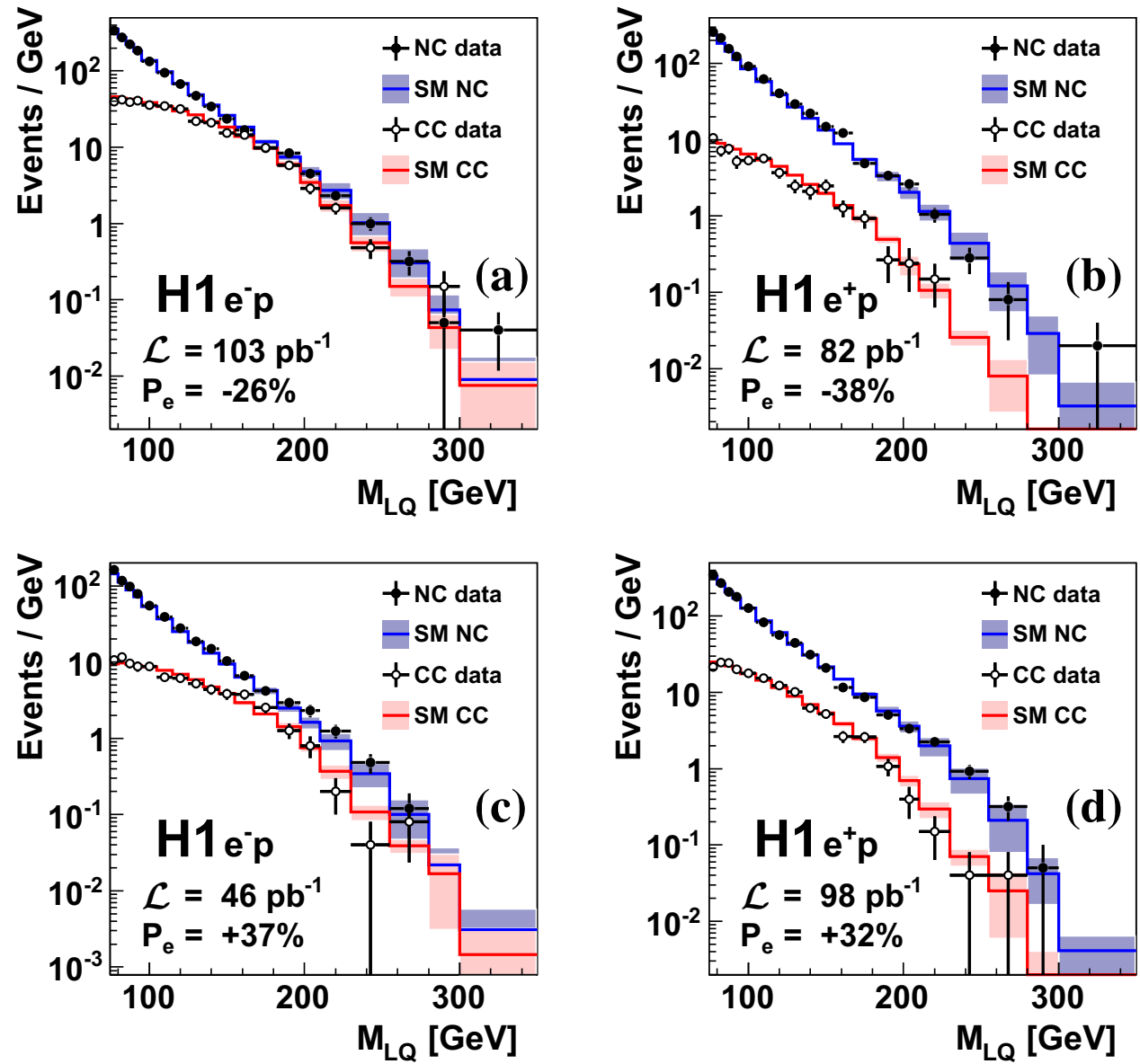

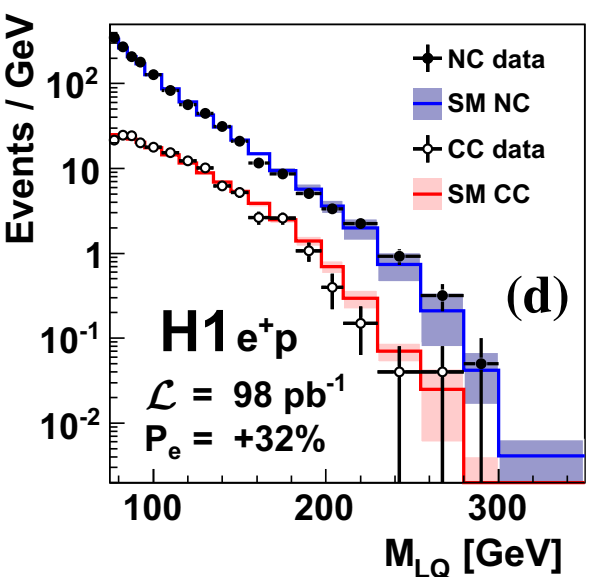

where both the NC-like and CC-like event samples are presented [148]. The shape and normalisation of all samples are well described. Similarly good agreement is observed in the equivalent mass distributions in the ZEUS analysis [149].

Figure 22 shows as an example the left-handed $e^{-} p$ ZEUS data without (top) and with (bottom) the $\cos \theta^{*}<0.4$ analysis cut applied. In both cases good agreement is seen between the data and the SM NC prediction. Also shown are two predictions for a $S_{0}^{L}$ LQ state with a mass of $210 \mathrm{GeV}$ and coupling strength $\lambda=0.3$ and a mass of $400 \mathrm{GeV}$ and a coupling strength $\lambda=1.0$.

Since no evidence for LQ production is observed in any of the NC or CC data samples, the data are used to set constraints on leptoquarks coupling to first generation fermions. To estimate the LQ signal, an event reweighting technique is applied to the SM MC events. The reweighting procedure uses the CTEQ5 [150] PDF for the LQ signal. For the limit analysis, the data are studied in bins by $\mathrm{H} 1$ (ZEUS) in the $M_{\mathrm{LQ}}-y\left(M_{l j s}-\cos \theta^{*}\right)$ plane, where the NC and CC data samples with different lepton-beam charge and polarisation are kept as distinct data sets. Limits are determined from a statistical analysis which uses the method of fractional event counting [151], taking into account the polarisation and systematic uncertainties, the most relevant of which are the electromagnetic and hadronic energy scales and the PDF uncertainty. Full details on the limit calculation employed can be found in the individual publications [148, 149].

Upper limits from ZEUS on the coupling $\lambda$ obtained at $95 \% \mathrm{CL}$ are shown as a function of leptoquark mass in Fig. 23, displayed as groups of scalar and vector LQs for both $F=2$ and $F=0$.

Limits corresponding to LQs coupling to a $u$ quark are better compared to those for LQs coupling to the $d$ quark only, as expected from the larger $u$ quark density in the proton. Corresponding to the steeply falling parton density function for high values of $x$, the LQ production cross section decreases rapidly and exclusion limits from HERA are less stringent towards higher LQ masses. For LQ masses near the kinematic limit of $319 \mathrm{GeV}$, the limit corresponding to a resonantly produced LQ turns smoothly into a limit on the virtual effects of both an off-shell $s$-channel LQ process and a $u$-channel LQ exchange.

The presented limits extend beyond those from previous leptoquark and contact interaction analyses by H1 [125, 142] 

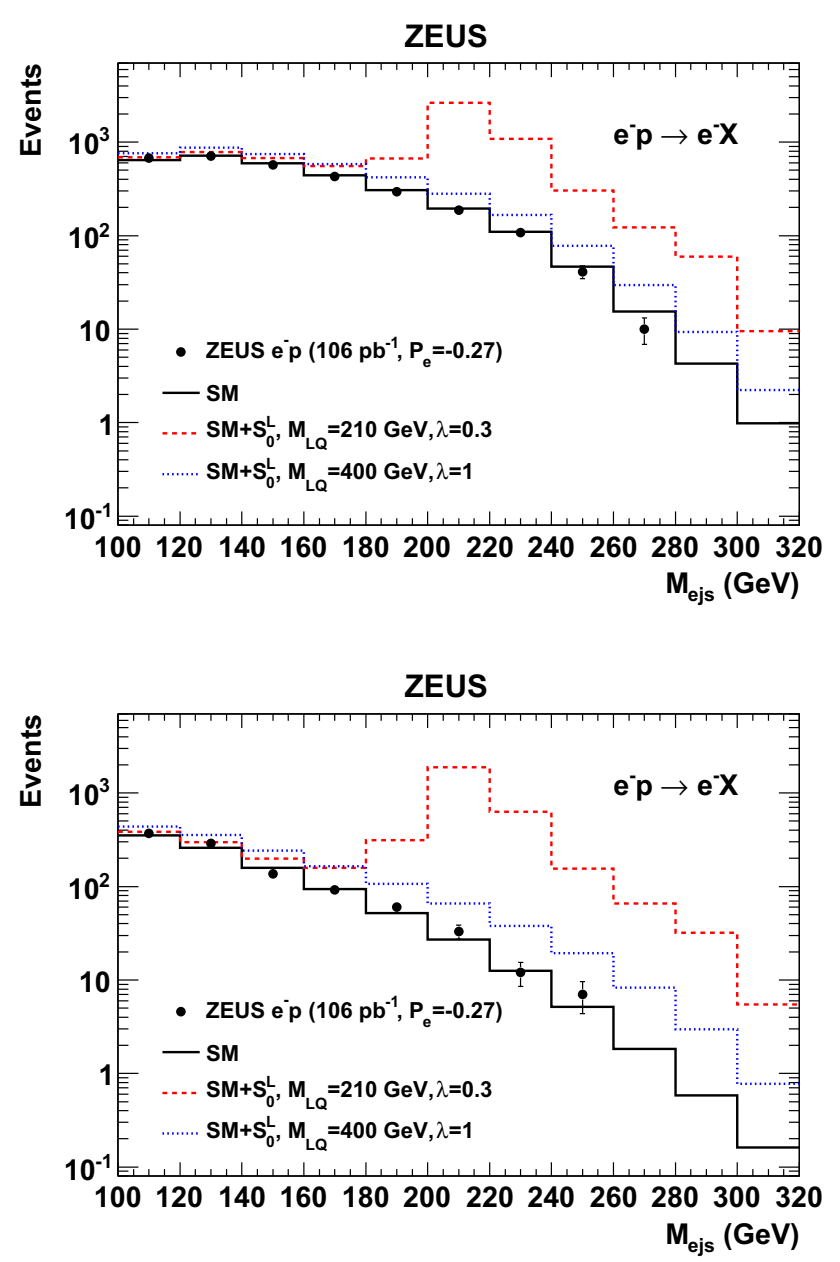

Fig. 22 Top the reconstructed invariant mass, $M_{e j s}$, distribution in the $e^{-} p \rightarrow e^{-} X$ topology for the left-handed $e^{-} p$ ZEUS sample (dots), compared to the SM prediction (solid histogram) and to the predictions of the BRW-based LQ model including a $S_{0}^{L}$ LQ state with a mass of $210 \mathrm{GeV}$ with a coupling strength $\lambda=0.3$ (dashed histogram) and a mass of $400 \mathrm{GeV}$ and a coupling $\lambda=1.0$ (dotted histogram). Bottom the same distribution after the additional analysis cut on $\cos \theta^{*}<0.4$ is applied

and ZEUS $[120,145]$ based on smaller HERA data sets. For a coupling of electromagnetic strength $\lambda=\sqrt{4 \pi \alpha_{\mathrm{em}}}=0.3$, LQs produced in $e p$ collisions decaying to an electron-quark or a neutrino-quark pair are excluded at $95 \% \mathrm{CL}$ up to leptoquark masses between $290 \mathrm{GeV}\left(\tilde{S}_{0}^{R}\right)$ and $699 \mathrm{GeV}$ $\left(V_{1}^{L}\right)$, depending on the leptoquark type. Similar limits are derived by $\mathrm{H} 1$, where LQs decaying to an electron-quark or a neutrino-quark pair are excluded at $95 \% \mathrm{CL}$ up to leptoquark masses between $277 \mathrm{GeV}\left(V_{0}^{R}\right)$ and $800 \mathrm{GeV}\left(V_{0}^{L}\right)$, depending on the leptoquark type.

Within the framework of the BRW model, the $\tilde{S}_{1 / 2}^{L}$ LQ decays exclusively to an electron-quark pair, resulting in a branching fraction for decays into charged leptons of $\beta_{\ell}=1.0$, whereas the $S_{0}^{L}$ LQ also decays to a neutrinoquark pair, resulting in $\beta_{\ell}=0.5$. The $\mathrm{H} 1$ and ZEUS limits on $\tilde{S}_{1 / 2}^{L}$ and $S_{0}^{L}$ from the analysis of the full HERA data are compared to those from other experiments in Fig. 24 The H1 limits at high leptoquark mass values are also compared with those obtained in the contact interaction analysis described in Sect. 9, which is based on single differential NC cross sections $\mathrm{d} \sigma / \mathrm{d} Q^{2}$ measured using the same data. The additional impact of the CC data can be seen in the case of the $S_{0}^{L} \mathrm{LQ}$, where a stronger limit is achieved in this analysis, whereas for the $\tilde{S}_{1 / 2}^{L}$ LQ the two analyses result in a similar limit.

Indirect limits from searches for new physics in $e^{+} e^{-}$ collisions at LEP by the OPAL [152] and L3 [153] experiments are indicated, as well as the limits from the DØ [154, 155] experiment at the Tevatron and from searches by the ATLAS [156] and CMS [157] experiments based on their $\sqrt{s}=7 \mathrm{TeV}$ data. The limits from hadron colliders are primarily based on searches for LQ pair-production and are independent of the coupling $\lambda$. For example, for a leptoquark mass of $640 \mathrm{GeV}$, the H1 LQ analysis rules out the $S_{0}^{L} \mathrm{LQ}$ for coupling strengths larger than about 0.35 .

At the time of writing in winter 2015-2016, results from the $\sqrt{s}=8 \mathrm{TeV}$ data LHC are appearing, extending these limits into the kinematic regime beyond $1 \mathrm{TeV}$ [158] for $\beta=1.0$ leptoquarks. Limits from the LHC experiments so far refer only to scalar leptoquarks.

\subsection{Search for second and third generation leptoquarks}

The introduction of lepton flavour violation [159] to leptoquark models would mean that the processes $e p \rightarrow \mu X$ or $e p \rightarrow \tau X$, mediated by the exchange of a second or third generation leptoquark, would be observable at HERA with final states containing a muon or the decay products of a tau lepton back-to-back in the transverse plane with a hadronic system $X$. Searches for such signatures have been previously performed at HERA by both H1 and ZEUS using HERA I data $[140,141,160]$. The analysis performed by H1 using their complete $\sqrt{s}=319 \mathrm{GeV}$ data set (see Table 1) is described in the following. The event selections for both the searches are described below; full details can be found in the publication [161].

Leptoquarks with couplings to first and second generation leptons may decay to a muon and a quark, therefore event topologies with an isolated, high transverse momentum muon back-to-back to a hadronic system in the transverse plane are selected. An initial sample of events with muons and jets is selected by requiring at least one $P_{T}^{\mu}>8 \mathrm{GeV}$ muon in the polar angular range $10^{\circ}<\theta_{\mu}<120^{\circ}$ and at least one jet. Events with isolated muons are then selected; the angular distance, $D=\sqrt{(\Delta \eta)^{2}+(\Delta \phi)^{2}}$, of the muon to the nearest track and to the nearest jet are required to be greater than 0.5 and 1.0, respectively. Limitations on the calorimetric energy in a cone around the muon are also introduced [161]. To 


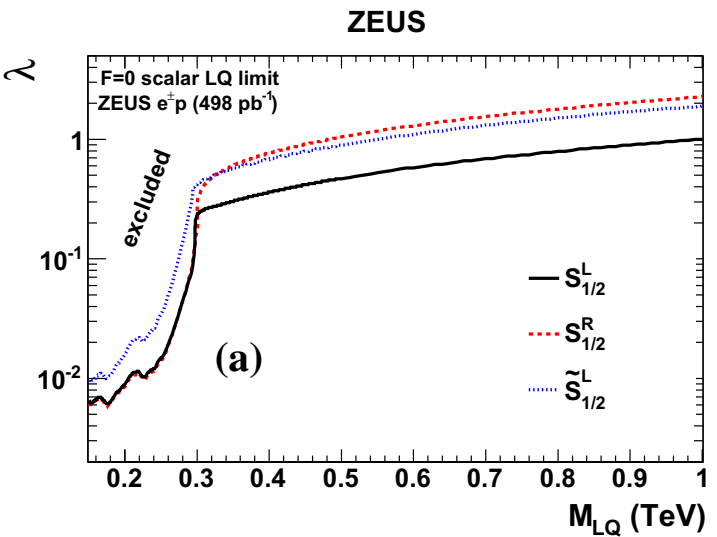

ZEUS

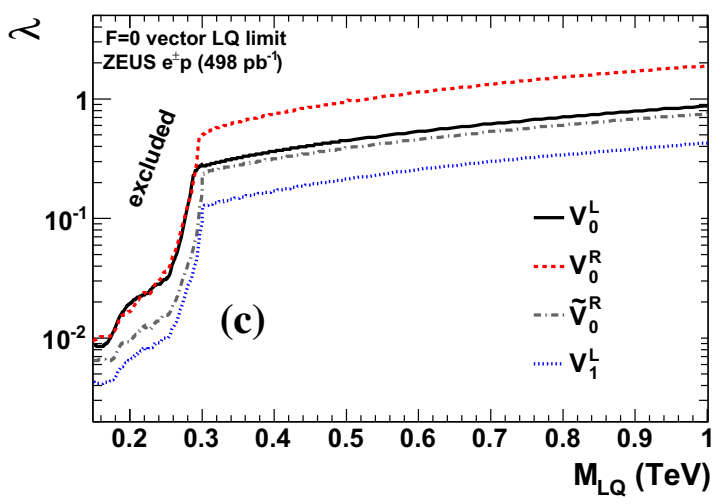

Fig. 23 Exclusion limits from ZEUS for the 14 leptoquarks in the framework of the Buchmüller, Rückl and Wyler model on the coupling $\lambda$ as a function of leptoquark mass for the scalar leptoquarks with $F=0$

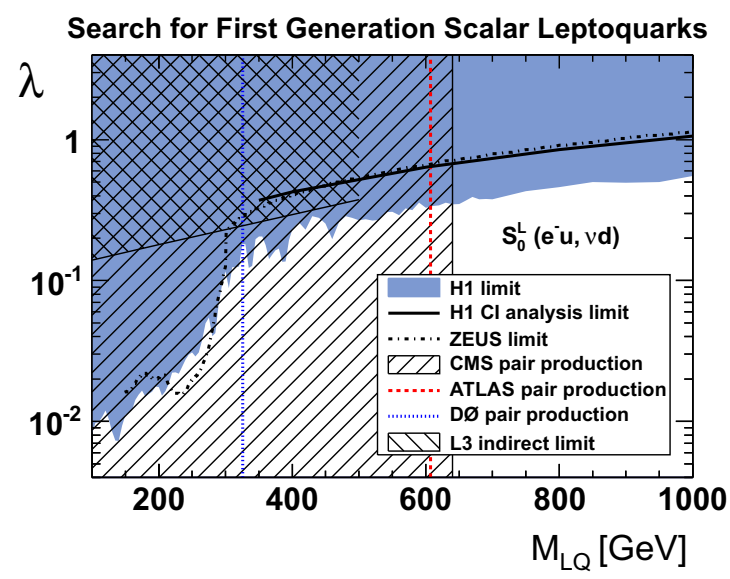

Fig. 24 Exclusion limits from H1 and ZEUS in the framework of the Buchmüller, Rückl and Wyler model on the coupling as a function of leptoquark mass for the $S_{0}^{L}$ (left) and $\tilde{S}_{1 / 2}^{L}($ right) leptoquarks, which have branching fraction to charged leptons of $\beta_{\ell}=0.5$ and $\beta_{\ell}=1.0$, respectively. Domains above the curves and to the left of the vertical lines are

reduce the muon-pair SM background exactly one isolated muon is required, as expected in LFV LQ signal events.

NC DIS background is suppressed by applying a cut on the calorimetric momentum imbalance, $P_{T}^{\text {calo }}>25 \mathrm{GeV}$,

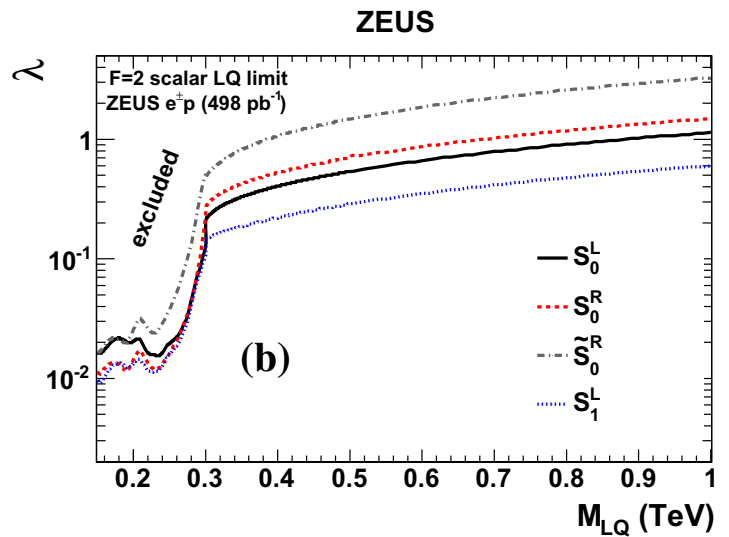

ZEUS

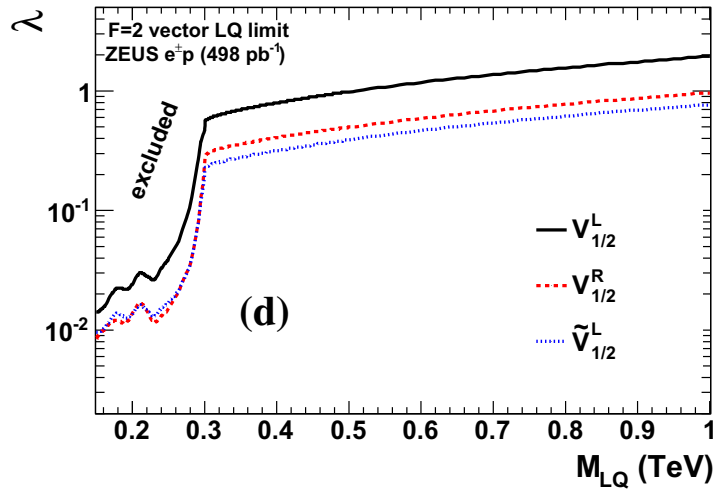

(a) and $F=2($ b) and the vector leptoquarks with $F=0$ (c) and $F=2$ (d). Domains above the curves are excluded at $95 \%$ CL

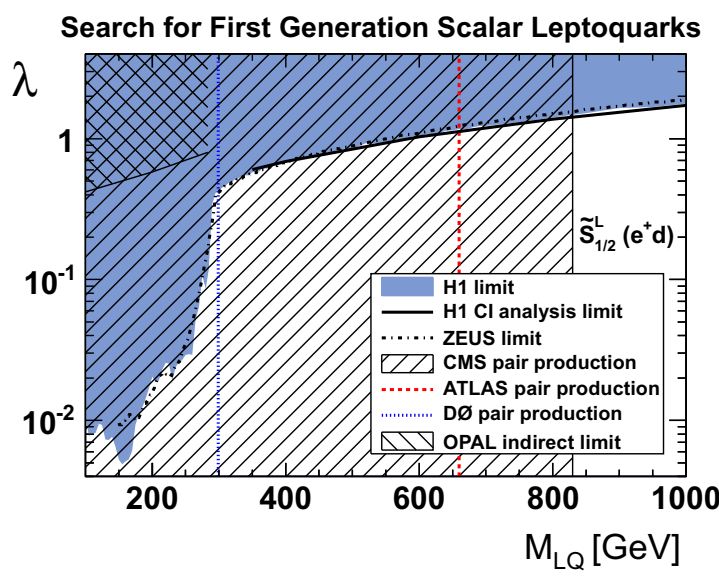

excluded at $95 \%$ CL. Published limits from the Tevatron (DØ), LEP(L3 and OPAL) and the LHC experiments (CMS and ATLAS, $\sqrt{s}=7 \mathrm{TeV}$ data) are also shown for comparison, as well as constraints on LQs with masses above $350 \mathrm{GeV}$ from the $\mathrm{H} 1$ contact interaction (CI) analysis

and by rejecting events with identified isolated electrons. The back-to-back event topology in the azimuthal plane is also exploited to remove the SM background: the difference between the azimuthal angle of the hadronic sys- 
Fig. 25 The reconstructed leptoquark mass in the $\mathrm{H} 1$ search for $e p \rightarrow \mu X$ (left) and $e p \rightarrow \tau X$ (right) events. The data are the points and the total uncertainty on the SM expectation (open histogram) is given by the shaded band. The dashed histogram indicates the LQ signal with arbitrary normalisation for a leptoquark mass of $150 \mathrm{GeV}$

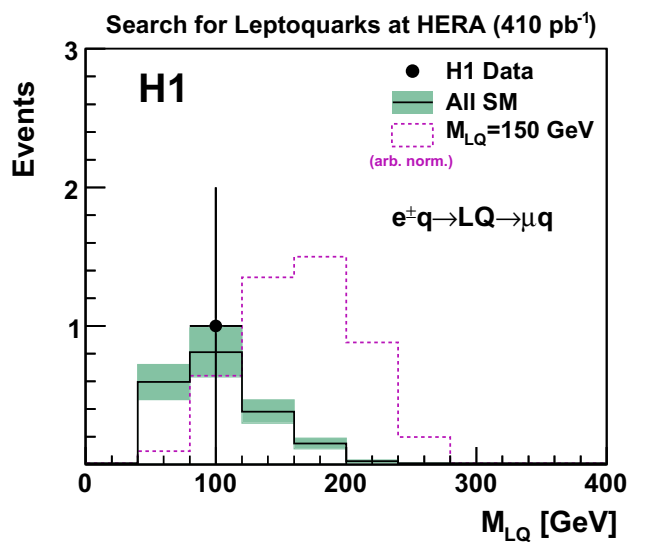

tem and the muon, the acoplanarity $\Delta \phi_{\mu-X}$, is required to be greater than $170^{\circ}$. Further SM background reduction is achieved by exploiting the calorimetric energy imbalance $V_{\mathrm{ap}} / V_{\mathrm{p}}<0.3$ and the overall longitudinal balance of the event $\delta>40 \mathrm{GeV}$.

After all selection cuts, in the analysis of $\mu X$ final states one event is observed in the data which compares well to the $\mathrm{SM}$ prediction of $2.0 \pm 0.4$. The largest contribution comes from muon-pair events, which are modelled by GRAPE.

Leptoquarks with couplings to first and third generation leptons may decay to a tau and a quark and in the search for third generation leptoquarks, tau leptons are identified using the muonic and one-prong hadronic decays of the tau. ${ }^{8}$ Hadronic tau decays, both one-prong and three-prong, are described in more detail in Sect. 11.2. Muonic tau decays, $\tau \rightarrow \mu v_{\mu} v_{\tau}$, result in final states similar to the high $P_{T}$ muon signatures described above and the same selection is therefore applied in that channel.

The one-prong hadronic decay of the tau leads to a high $P_{T}$, narrow "pencil-like" jet, so that the typical LFV signal event topology is a di-jet event. An initial di-jet event sample for the analysis of this decay channel is formed by selecting events with at least two jets in the polar angle range $5^{\circ}<$ $\theta_{\text {jet }}<175^{\circ}$ and with $P_{T}^{\text {jet1 }}>20 \mathrm{GeV}$ and $P_{T}^{\text {jet2 }}>15 \mathrm{GeV}$. The undetected neutrinos from tau lepton decays result in an overall $P_{T}$ imbalance and therefore a minimum missing transverse momentum $P_{T}^{\text {miss }}>12 \mathrm{GeV}$ is required.

A tau jet is characterised by a narrow energy deposit in the calorimeter and a low track multiplicity within the identification cone of the jet. Tau jets are identified in the di-jet sample, where the candidates are required to be in the polar angle range $20^{\circ}<\theta_{\text {jet }}<120^{\circ}$ and to have a maximum jet radius $R_{\text {jet }}$ of 0.12 [162], where

\footnotetext{
8 The electronic decays of the tau $\tau \rightarrow e v_{e} v_{\tau}$ result in final states very difficult to distinguish from SM NC DIS, where the missing transverse momentum is aligned with the electron. As such, they are not considered in the analysis.
}

$$
R_{\text {jet }}=\frac{1}{E_{\text {jet }}} \sum_{h} E_{h} \sqrt{\Delta \eta(\text { jet }, h)^{2}+\Delta \phi(\text { jet }, h)^{2}},
$$

and $E_{\text {jet }}$ is the total jet energy. The sum runs over all hadronic final-state particles in jets with energy $E_{h}$. At least one track with $P_{T}$ larger than $2 \mathrm{GeV}$ not associated with an identified electron or muon is required within the jet radius of the tau jet.

A single tau jet is required in the final sample, with selection requirements including isolation from tracks and other jets by an angular distance $D>1.0$, a track multiplicity of one in a cone of radius $R=1.0$ around the jet axis and a maximum of $90 \%$ of the jet energy in the EMC to reject purely electromagnetic jets.

Further cuts are then applied to reduce the remaining SM background: the hadronic transverse momentum $P_{T}^{h}$ is required to be larger than $30 \mathrm{GeV}$ and the acoplanarity between the tau jet and hadronic system in the transverse plane $\Delta \phi_{\tau-X}$ is required to be greater than $160^{\circ}$. Analogous to the muon channel, a cut of $\delta>40 \mathrm{GeV}$ is also applied to exploit the longitudinal balance of the event.

After all selection cuts, in the analysis of $\tau X$ final states where the tau lepton decays hadronically, 6 events are observed in the data, in good agreement with the SM prediction of $8.2 \pm 1.1$. The main SM contribution is from remaining NC DIS events, which are modelled by the RAPGAP [163] event generator.

The reconstructed leptoquark-candidate mass in the search for $e p \rightarrow \mu X$ and $e p \rightarrow \tau X$ events is shown in Fig. 25, compared to the SM prediction and an example LQ signal with arbitrary normalisation. The LQ kinematics are reconstructed using the double angle method (Eq. (15a)). The direction of the detected lepton and the hadronic final state are used to reconstruct the Bjorken scaling variable $x$ and subsequently the LQ mass following Eq. (40).

As the observed number of events is in agreement with the SM prediction and therefore no evidence for LFV is found, the results of the search are interpreted in terms of exclusion limits on the mass and the coupling of LQs mediating LFV. 
Fig. 26 Exclusion limits on the coupling constants $\lambda_{\ell q_{1}}=\lambda_{e q_{1}}$ as a function of leptoquark mass $M_{\mathrm{LQ}}$ for $F=0$ leptoquarks: limits on second generation $(\ell=\mu)$ scalar (a) vector $(\mathbf{b})$ LQs; limits on third generation $(\ell=\tau)$ scalar $(\mathbf{c})$ vector $(\mathbf{d})$ LQs. Regions above the lines are excluded at $95 \% \mathrm{CL}$. The notation $q_{1}$ indicates that only processes involving first generation quarks are considered. The parentheses after the LQ name indicate the fermion pairs coupling to the LQ, where pairs involving anti-quarks are not shown
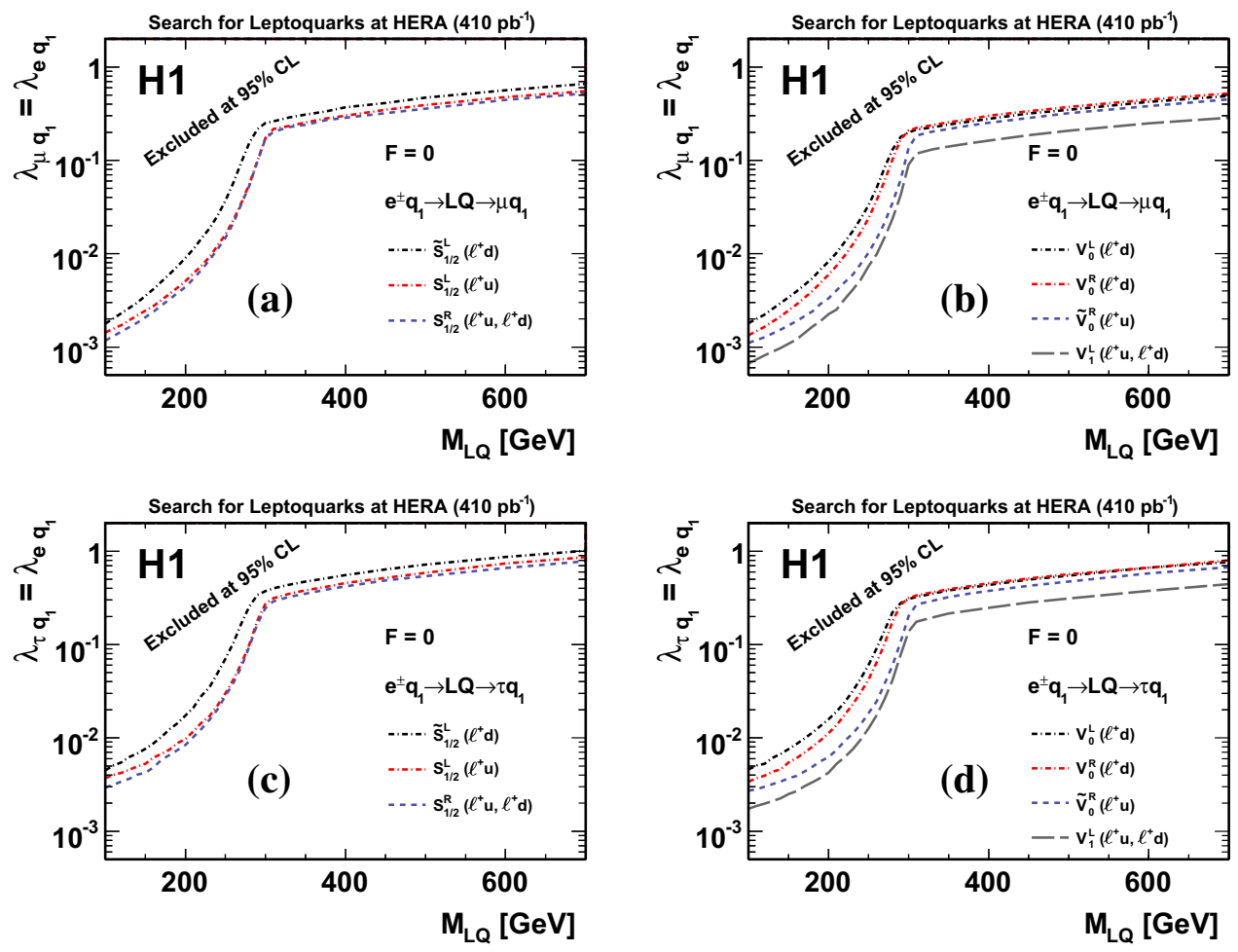

The LQ production mechanism at HERA involves a nonzero coupling to the first generation fermions $\lambda_{e q_{i}}>0$. For the LFV leptoquark decay, it is assumed that only one of the couplings $\lambda_{\mu q_{j}}$ and $\lambda_{\tau q_{j}}$ is non-zero and that $\lambda_{e q_{i}}=$ $\lambda_{\mu q_{j}}\left(\lambda_{\tau q_{j}}\right)$. A modified frequentist method with a likelihood ratio as the test statistic is used to combine the individual data sets and the ep $\rightarrow \tau X$ search channels [164]. In order to avoid the need to generate many signal MC samples at each leptoquark mass, coupling and branching ratio, a weighting technique is used to provide predictions across the full range of LQ production parameters [140]. As in the search for first generation leptoquarks, the lepton-beam polarisation enters the limit calculation for the HERA II data.

Figure 26 shows the $95 \%$ CL upper limits on the couplings to the first generation quarks, $\lambda_{\mu q_{1}}$ and $\lambda_{\tau q_{1}}$, for $F=0 \mathrm{LQs}$ as a function of the mass of the LQ leading to LFV in ep collisions. These limits extend beyond those from $\mathrm{H} 1$ and ZEUS based on the HERA I data alone [140,141,160]. Similar limits are found for $F=2 \mathrm{LQs}$ and these can be found in the $\mathrm{H} 1$ publication along with limits involving heavier quark flavours [161].

For $\lambda=\sqrt{4 \pi \alpha_{\mathrm{em}}}=0.3$, LFV leptoquarks produced in ep collisions decaying to a muon-quark or a tau-quark pair are excluded at $95 \%$ CL up to leptoquark masses of 712 and $479 \mathrm{GeV}$, respectively. In both cases, the LQ with the highest sensitivity is $V_{1}^{L}$. As described above, the most appropriate value for the branching ratio when comparing to results from hadron colliders is $\beta=0.5$ or less (see Eqs. (37)(39)). In a search for pair production of second generation scalar leptoquarks, the ATLAS Collaboration rules out leptoquark masses at $95 \% \mathrm{CL}$ less than about $850 \mathrm{GeV}$ for $\beta=0.5$ [158]. The CMS Collaboration has recently performed searches for third generation scalar LQ pairs decaying to $t+\tau$ [165] and $b+\tau$ [166] final states, where the best limit for $\beta=0.5$ is $M_{\mathrm{LQ}}>560 \mathrm{GeV}$ at $95 \% \mathrm{CL}$.

\section{Multi-lepton production at high transverse momentum}

Multi-lepton final states may be produced in electron-proton collisions at HERA, proceeding mainly via photon-photon $\gamma \gamma \rightarrow \ell^{+} \ell^{-}$interactions [167], as shown in Fig. 27. The dominant contribution, shown in Fig. 27a, is from lepton pair production via the interaction of two photons radiated from the incident electron and proton. Lepton pairs may also originate from internal conversion of a photon or a $Z^{0}$ boson, radiated either from the incident electron line (Fig. 27b) or from the quark line (Fig. 27c).

As this is a purely Quantum Electrodynamic (QED) process, the cross section is precisely calculable in the SM. Multi-lepton events are simulated using the GRAPE event generator, which includes all leading-order electroweak matrix elements. The final-state leptons provide a clean event signature, which is well described by the SM and the investigation of the high-mass, high- $P_{T}$ regions, where the SM expectation is low, may reveal some signal of new physics. 
Fig. 27 Lepton pair production in $e^{+} p$ collisions at HERA. Examples of Feynman diagrams are shown for the photon-photon interaction (a) and $\gamma / Z^{0}$ conversion $\mathbf{b}$ and $\mathbf{c}$. The hadronic final state can be a proton (elastic production) or a higher mass system (quasi-elastic and inelastic production)

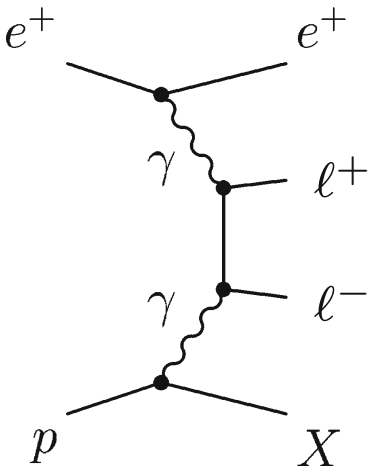

(a)

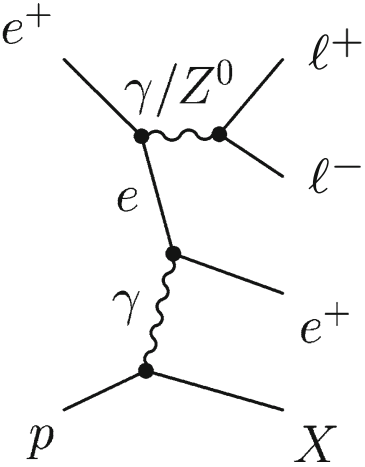

(b)

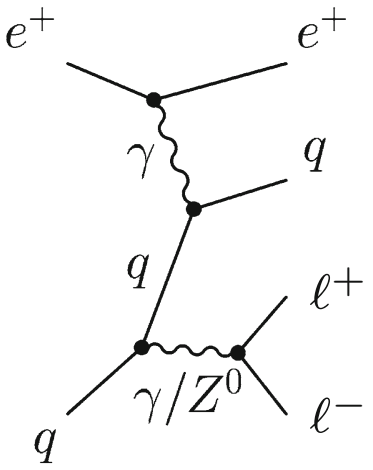

(c)
The following section concerns final states with electrons and muons. Studies of tau-pair production are described in Sect. 11.2.

\subsection{Events with electron and muon final states}

Measurements of both multi-electron [168] and muon pair [169] production at high transverse momentum have previously been performed by H1 using their HERA I data set. Since then, both H1 and ZEUS have published [170,171] their final results on the search for multi-lepton events, using the full available statistics of the complete HERA data set. The integrated luminosity in the $\mathrm{H} 1$ analysis corresponds to $463 \mathrm{pb}^{-1}$, of which $285 \mathrm{pb}^{-1}$ are from $e^{+} p$ collisions and $178 \mathrm{pb}^{-1}$ from $e^{-} p$ collisions. In the ZEUS analysis, the integrated luminosity corresponds to $480 \mathrm{pb}^{-1}$, of which $278 \mathrm{pb}^{-1}$ are from $e^{+} p$ collisions and $202 \mathrm{pb}^{-1}$ from $e^{-} p$ collisions.

The analysis strategy and event selection used in the H1 and ZEUS analyses are similar. First, electron or muon candidates are identified in a wide angular region using a loose selection criterion. Then, at least two central $\left(20^{\circ}<\theta_{\ell}<\right.$ $150^{\circ}$ ) lepton candidates are required in the event. Electrons are identified in the polar angle range $5^{\circ}<\theta_{e}<175^{\circ}$ with an energy greater $E_{e}>5 \mathrm{GeV}$. This energy threshold is raised to $10 \mathrm{GeV}$ in the region $5^{\circ}<\theta_{e}<20^{\circ}$ in the $\mathrm{H} 1$ analysis and in the region $5^{\circ}<\theta_{e}<150^{\circ}$ in the ZEUS analysis. Muons are identified in the polar angle range $20^{\circ}<\theta_{\mu}<160^{\circ}$ and are required to have transverse momentum $P_{T}^{\mu}>2 \mathrm{GeV}$.

The final event selection requires at least two central lepton candidates, one with $P_{T}>10 \mathrm{GeV}$ and the other with $P_{T}>$ $5 \mathrm{GeV}$. Additional leptons identified according to the above criteria may also be present in the event. The leptons are required to be isolated from each other by an angular distance $D>0.5$ in the $\eta-\phi$ plane. According to the number and flavours of the identified leptons, the events are classified into mutually exclusive topologies containing up to 4 leptons.

The main source of SM background in each analysis depends on the number and flavour of the identified lep- tons in the event sample. NC DIS and QED Compton scattering (QEDC, $e p \rightarrow e \gamma X$ ) constitute a significant background only for event topologies in which two leptons, one of which is an electron, are found in the final state. The NC DIS background is modelled in the H1 (ZEUS) analysis using the RAPGAP (DJANGOH) event generator. The smaller QEDC background contribution is modelled using WABGEN [172] (GRAPE) in the H1 (ZEUS) analysis. Background contributions to the SM expectation are negligible in events in which two muons or more than two leptons are observed.

The number of selected events in the $\mathrm{H} 1$ analysis [170] in the various topologies are compared to SM predictions in Table 5, where good agreement is observed for all event samples. In the table, the number of events from genuine pair production is shown, together with the number of events from NC DIS and QED Compton, which constitute the most significant source of background. Events with high invariant mass of the two highest $P_{T}$ leptons $M_{12}$ are observed in the data, where the SM expectation is low. All events with $M_{12}>100 \mathrm{GeV}$ are seen in the $e^{+} p$ data only. Two of the high-mass events observed by $\mathrm{H} 1$ are shown in Fig. 28. A high scalar sum of the lepton transverse momenta $\sum P_{T} \mathrm{GeV}$, which is summed over all identified leptons in the event, may be an indication of new physics and five events are seen in the $\mathrm{H} 1$ data, compared to a SM expectation of $1.60 \pm 0.20$. All events are seen in the $e^{+} p$ data, where $0.96 \pm 0.12$ are expected from the SM. The $\sum P_{T} \mathrm{GeV}$ distribution from the H1 analysis is shown in Fig. 29.

Similarly for the ZEUS analysis [171], the number of selected events in the various topologies studied are compared to the SM prediction in Table 5, where a good agreement is observed for all event samples. The $\sum P_{T}$ and $M_{12}$ distributions of all topologies combined are shown in Fig. 29, where the shape and normalisation are well described by the SM predictions. Some interesting events are also observed in the ZEUS data at high- $M_{12}$ and high- $\sum P_{T}$ : in particular, two events are present in the data with $\sum P_{T}>100 \mathrm{GeV}$ compared to a SM prediction of $1.56 \pm 0.15$. Two of 
Table 5 Observed and predicted multi-lepton event yields for the different event topologies in the H1, ZEUS and combined analysis. The total SM event yield is given by the sum of the signal, lepton pair production in $\gamma \gamma$ interactions, and the background, mainly from NC DIS and QEDC events. For the combined analysis, performed in a common phase space, the event yields shown for the $\gamma \gamma$ subsamples are those used in the cross section measurement. The uncertainties on the predictions include model uncertainties and experimental systematic uncertainties added in quadrature. The limits on the background estimations are quoted at $95 \% \mathrm{CL}$

\begin{tabular}{|c|c|c|c|c|}
\hline \multicolumn{5}{|c|}{ Searches for multi-lepton events at HERA } \\
\hline Event sample & Data & Total SM & Pair production & $\mathrm{NC}$ DIS + QEDC \\
\hline \multicolumn{5}{|c|}{$\mathrm{H} 1$ analysis $\left(\mathcal{L}=463 \mathrm{pb}^{-1}\right)$} \\
\hline$e e$ & 368 & $390 \pm 46$ & $332 \pm 26$ & $58 \pm 30$ \\
\hline$\mu \mu$ & 201 & $211 \pm 32$ & $211 \pm 32$ & $<0.005$ \\
\hline$e \mu$ & 132 & $128 \pm 9$ & $118 \pm 8$ & $10.0 \pm 2.5$ \\
\hline eee & 73 & $70 \pm 7$ & $69.8 \pm 7.0$ & $0.2 \pm 0.1$ \\
\hline$e \mu \mu$ & 97 & $102 \pm 14$ & $102 \pm 14$ & $<0.005$ \\
\hline$e e \mu$ & 4 & $1.43 \pm 0.26$ & $1.18 \pm 0.20$ & $0.25 \pm 0.14$ \\
\hline eeee & 1 & $0.33 \pm 0.07$ & $0.33 \pm 0.07$ & $<0.005$ \\
\hline \multicolumn{5}{|c|}{ ZEUS analysis $\left(\mathcal{L}=480 \mathrm{pb}^{-1}\right)$} \\
\hline$e e$ & 545 & $563_{-37}^{+29}$ & $429_{-29}^{+21}$ & $134 \pm 11$ \\
\hline$\mu \mu$ & 93 & $106 \pm 12$ & $106 \pm 12$ & $<0.5$ \\
\hline$e \mu$ & 46 & $42 \pm 4$ & $37_{-4}^{+3}$ & $4.5 \pm 2$ \\
\hline eee & 73 & $75_{-4}^{+5}$ & $73_{-5}^{+4}$ & $<4$ \\
\hline$e \mu \mu$ & 47 & $48 \pm 5$ & $48 \pm 5$ & $<0.5$ \\
\hline eeee & 1 & $0.9_{-0.1}^{+0.5}$ & $0.6 \pm 0.1$ & $<1.4$ \\
\hline$e е \mu \mu$ & 2 & $0.5_{-0.1}^{+0.3}$ & $0.4 \pm 0.1$ & $<0.5$ \\
\hline \multicolumn{5}{|c|}{ Combined $\mathrm{H} 1$ and ZEUS analysis $\left(\mathcal{L}=0.94 \mathrm{fb}^{-1}\right)$} \\
\hline$e e$ & 873 & $895 \pm 57$ & $724 \pm 41$ & $171 \pm 28$ \\
\hline$\mu \mu$ & 298 & $320 \pm 36$ & $320 \pm 36$ & $<0.5$ \\
\hline$e \mu$ & 173 & $167 \pm 10$ & $152 \pm 9$ & $15 \pm 3$ \\
\hline eee & 116 & $119 \pm 7$ & $117 \pm 6$ & $<4$ \\
\hline$e \mu \mu$ & 140 & $147 \pm 15$ & $147 \pm 15$ & $<0.5$ \\
\hline$(\gamma \gamma)_{e}$ & 284 & $293 \pm 18$ & $289 \pm 18$ & $4 \pm 1$ \\
\hline$(\gamma \gamma)_{\mu}$ & 235 & $247 \pm 26$ & $247 \pm 26$ & $<0.5$ \\
\hline
\end{tabular}

the highest mass events observed by ZEUS are shown in Fig. 28.

A combination of the $\mathrm{H} 1$ and ZEUS multi-lepton analyses has also been performed, using a data sample with a total integrated luminosity of $0.94 \mathrm{fb}^{-1}$ [173]. Five of the final-state topologies are combined, namely $е e, \mu \mu, e \mu$, eеe and $e \mu \mu$. The combination is performed in a common phase space, corresponding to a tightening of the selection cuts of the two experiments. For example, in the $\mathrm{H} 1$ analysis the electron energy threshold in the central region was raised from 5 to $10 \mathrm{GeV}$. Both the number of the observed events and the cross sections for multi-lepton production measured by the two experiments were combined. This allows a better sensitivity to rare processes in the high $M_{12}$ and high $\sum P_{T}$ regions to be achieved and an improved precision of the measured cross sections.

The event yields of the combined analysis are shown in Table 5, where good agreement is observed with the SM.
The $\sum P_{T}$ distributions for the full combined $e^{ \pm}$data, as well as separately for the $e^{+} p$ and $e^{-} p$ data, are shown in Fig. 30. In general, good agreement is found between the data and the SM predictions. For $\sum P_{T}>100 \mathrm{GeV}$, seven data events are observed in total, compared to $3.13 \pm 0.26$ expected from the SM. These seven events were all recorded in $e^{+} p$ data, for which the SM expectation is $1.94 \pm 0.17$. Events are observed in all topologies with $M_{12}>100 \mathrm{GeV}$, as detailed in Table 6 . Both experiments recorded these events in $e^{+} p$ collisions only [173].

The cross sections for lepton pair production were also measured by both collaborations $[170,171]$ in the photoproduction regime, in which the virtuality $Q^{2}$ of the photon emitted by the beam lepton is low. Photoproduction events were selected by requiring a total $E-P_{z}$ of $\delta<45 \mathrm{GeV}$, which singles out events in which the scattered lepton is lost in the beam pipe and corresponds to phase-space cuts of $Q^{2}<1 \mathrm{GeV}^{2}$ and $y<0.82$. The event yield in this cross 

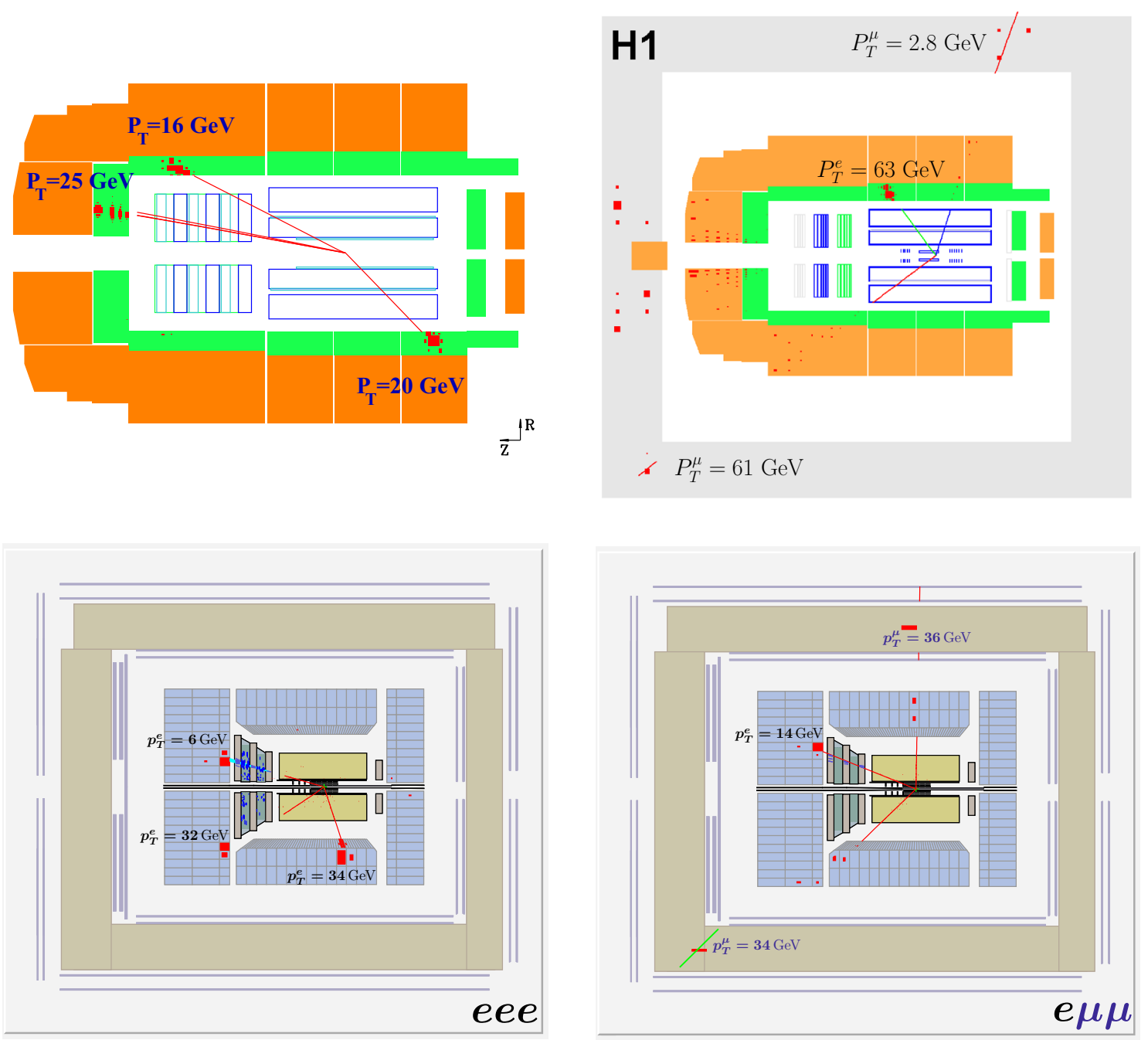

Fig. 28 Events observed in the H1 (top) and ZEUS (bottom) multilepton analyses. The measured transverse momentum of the leptons is indicated. Top left an event observed by $\mathrm{H} 1$ in the HERA I data containing three electrons. The invariant mass of the two highest $P_{T}$ electrons is measured as $M_{12}=118 \mathrm{GeV}$. Top right an $e \mu \mu$ event observed in

the H1 HERA II data where $M_{12}=127 \mathrm{GeV}$, formed by the electron and one of the muons. Bottom left a three electron event observed by ZEUS with $M_{12}=113 \mathrm{GeV}$. Bottom right an $e \mu \mu$ event observed by ZEUS where the muon pair form an invariant mass $M_{12}=77 \mathrm{GeV}$
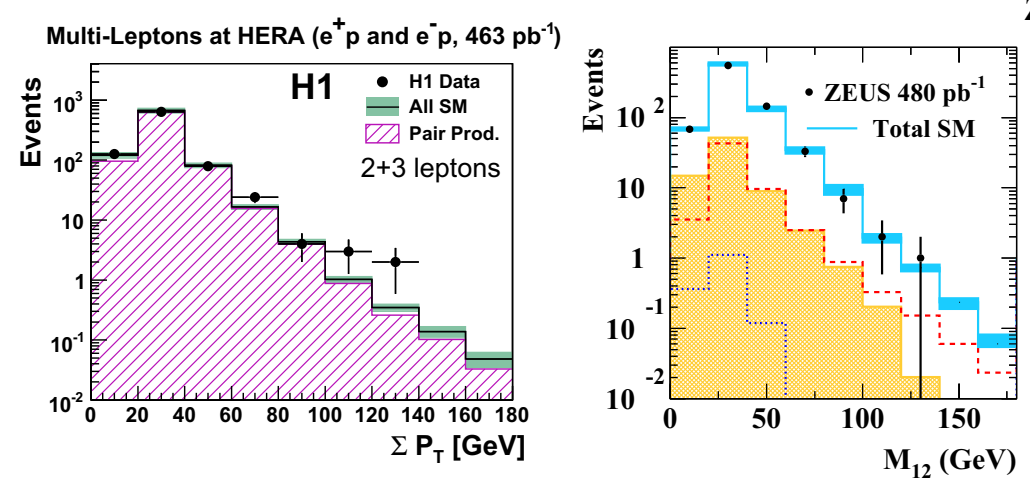

\section{ZEUS}

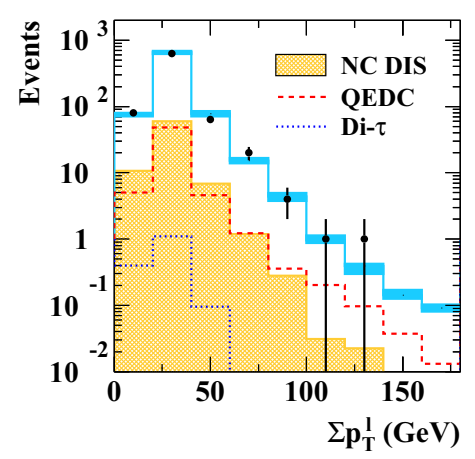

Fig. 29 The distribution of the scalar sum of the transverse momenta of all the leptons in the multi-lepton final states from the H1 (left) and ZEUS (right) analyses, as well as the invariant mass of the two highest$P_{T}$ leptons in the ZEUS analysis (centre), for the complete data sets and for all lepton topologies combined. The points correspond to the observed data events and the histogram to the SM expectation. The total uncertainty on the SM expectation is given by the shaded band. The individual components of the SM are as indicated within the figures 

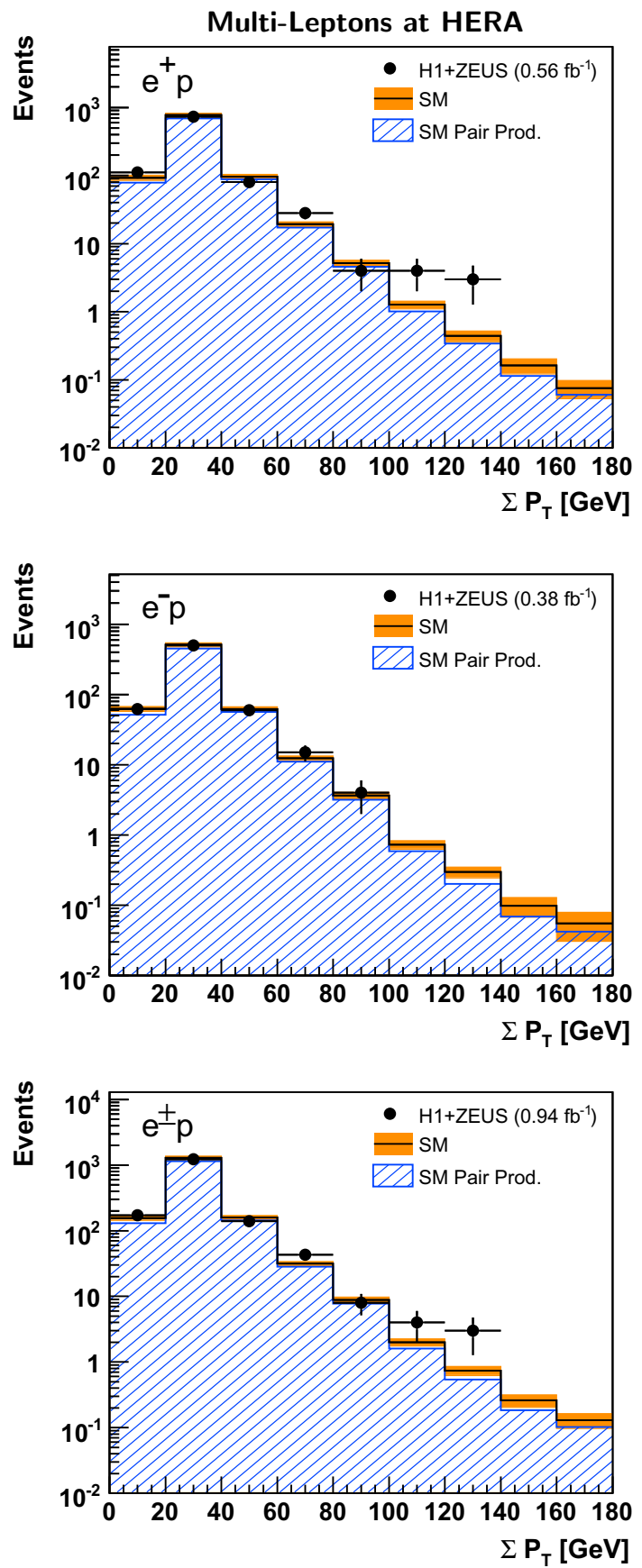

Fig. 30 The scalar sum of the transverse momentum $\sum P_{T}$ for combined di-lepton and tri-lepton event topologies for $e^{+} p$ (top), $e^{-} p$ (middle) data and for all data (bottom) in the combined H1 and ZEUS analysis. The points correspond to the observed data events and the histogram to the SM expectation. The total uncertainty on the SM expectation is given by the shaded band. The component of the SM expectation arising from lepton pair production is given by the striped histogram

section phase space is also presented for the combined analysis in Table 5 in the rows marked $(\gamma \gamma)_{e}$ and $(\gamma \gamma)_{\mu}$. The cross section is evaluated in each bin $i$ using the formula

$\sigma_{i}=\frac{N_{i}^{\text {data }}-N_{i}^{\mathrm{bgr}}}{\mathcal{L} \cdot A_{i}}$ where $N_{i}^{\text {data }}$ is the number of observed events in bin $i, N_{i}^{\mathrm{bgr}}$ the expected contribution from background processes in bin $i, \mathcal{L}$ the integrated luminosity of the data and $A_{i}$ is the signal acceptance in bin $i$ and is calculated using GRAPE.

The combined H1-ZEUS cross section measurement is evaluated using a weighted mean of the values measured by the two collaborations [173]. The total visible electron pair production cross section for the process $e p \rightarrow e e^{+} e^{-} X$ is measured in the restricted phase space as

$\sigma_{e^{+} e^{-}}=0.68 \pm 0.04$ (stat.) \pm 0.03 (sys.) pb,

where the first uncertainty is statistical and the second systematic. The total visible muon pair production cross section for the process $e p \rightarrow e \mu^{+} \mu^{-} X$ is measured as

$\sigma_{\mu^{+} \mu^{-}}=0.63 \pm 0.05$ (stat.) \pm 0.06 (sys.) pb.

As the SM cross sections for $e^{+} e^{-}$and $\mu^{+} \mu^{-}$production in $\gamma \gamma$ interactions are expected to be the same, the electron and muon pair production cross sections given above are combined into a single visible lepton pair production cross section of

$\sigma_{\ell^{+} \ell^{-}}=0.66 \pm 0.03$ (stat.) \pm 0.03 (sys.) pb,

which is in good agreement both with the SM prediction from GRAPE of $0.69 \pm 0.02 \mathrm{pb}$, as well as the individual H1 and ZEUS measurements [173]. The differential cross sections for lepton pair photoproduction as a function of the transverse momentum of the leading lepton, $P_{T}^{\ell_{1}}$, and of the invariant mass of the lepton pair, $M_{\ell \ell}$, are also measured and found to be in good agreement with SM predictions, as shown in Fig. 31.

\subsection{Tau-pair production}

The dominant tau-pair production mechanism is the same as for multi-electron and multi-muon production, $\gamma \gamma \rightarrow$ $\tau^{+} \tau^{-}$, as illustrated in Fig. 27. Tau particles can decay into leptons, $\tau \rightarrow \mu v_{\mu} \nu_{\tau}$, or $\tau \rightarrow e v_{e} \nu_{\tau}$, or into hadrons, $\tau \rightarrow h \nu_{\tau}$, which happens about $2 / 3$ of the time. Therefore, whilst the search of tau-pair production has the same physical motivation of that of multi-leptons, it is performed with different experimental techniques due to the different experimental signature of the tau lepton.

The tau-pair decay is classified as leptonic if both tau leptons decay leptonically, semi-leptonic if one tau lepton decays hadronically and one leptonically, and hadronic if both tau leptons decay into hadrons. From the experimental point of view, leptons from a tau decay cannot be distinguished from prompt electron or muon production, as the accompanying neutrino is not detected and hence events in which the two taus decay to leptons of the same flavour are rejected when examining tau-pair production. 
Table 6 Combined H1 and ZEUS analysis of multi-lepton events at HERA with $M_{12}>100 \mathrm{GeV}$. Event yields are shown for all data and divided into $e^{+} p$ and $e^{-} p$ collisions. The uncertainties on the predictions include model uncertainties and experimental systematic uncer- tainties added in quadrature. The limits on the background estimations correspond to the selection of no event in the simulated topology and are quoted at $95 \% \mathrm{CL}$

\begin{tabular}{|c|c|c|c|c|}
\hline \multicolumn{5}{|c|}{ Multi-lepton events at HERA with $M_{12}>100 \mathrm{GeV}$} \\
\hline \multicolumn{5}{|c|}{ Combined $\mathrm{H} 1$ and ZEUS analysis } \\
\hline Event sample & Data & Total SM & Pair production & NC DIS + QEDC \\
\hline \multicolumn{5}{|c|}{$e^{+} p$ collisions $\left(\mathcal{L}=0.56 \mathrm{fb}^{-1}\right)$} \\
\hline$e e$ & 4 & $1.68 \pm 0.18$ & $0.94 \pm 0.11$ & $0.74 \pm 0.12$ \\
\hline$\mu \mu$ & 1 & $0.32 \pm 0.08$ & $0.32 \pm 0.08$ & $<0.01$ \\
\hline$e \mu$ & 1 & $0.40 \pm 0.05$ & $0.39 \pm 0.05$ & $<0.02$ \\
\hline eee & 4 & $0.79 \pm 0.09$ & $0.79 \pm 0.09$ & $<0.03$ \\
\hline$e \mu \mu$ & 2 & $0.16 \pm 0.04$ & $0.16 \pm 0.04$ & $<0.01$ \\
\hline \multicolumn{5}{|c|}{$e^{-} p$ collisions $\left(\mathcal{L}=0.38 \mathrm{fb}^{-1}\right)$} \\
\hline$e e$ & 0 & $1.25 \pm 0.13$ & $0.71 \pm 0.11$ & $0.54 \pm 0.08$ \\
\hline$\mu \mu$ & 0 & $0.23 \pm 0.10$ & $0.23 \pm 0.10$ & $<0.01$ \\
\hline$e \mu$ & 0 & $0.26 \pm 0.03$ & $0.25 \pm 0.03$ & $<0.02$ \\
\hline eee & 0 & $0.49 \pm 0.07$ & $0.49 \pm 0.07$ & $<0.03$ \\
\hline$e \mu \mu$ & 0 & $0.14 \pm 0.05$ & $0.14 \pm 0.05$ & $<0.01$ \\
\hline \multicolumn{5}{|c|}{$e^{ \pm} p$ collisions $\left(\mathcal{L}=0.94 \mathrm{fb}^{-1}\right)$} \\
\hline$e e$ & 4 & $2.93 \pm 0.28$ & $1.65 \pm 0.16$ & $1.28 \pm 0.18$ \\
\hline$\mu \mu$ & 1 & $0.55 \pm 0.12$ & $0.55 \pm 0.12$ & $<0.01$ \\
\hline$e \mu$ & 1 & $0.65 \pm 0.07$ & $0.64 \pm 0.06$ & $<0.02$ \\
\hline eee & 4 & $1.27 \pm 0.12$ & $1.27 \pm 0.12$ & $<0.03$ \\
\hline$e \mu \mu$ & 2 & $0.31 \pm 0.06$ & $0.31 \pm 0.06$ & $<0.01$ \\
\hline
\end{tabular}

Multi-Leptons at HERA

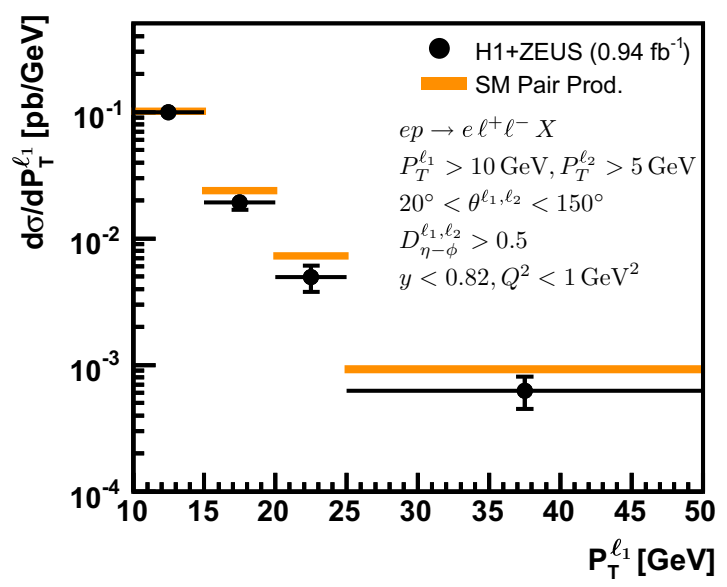

Fig. 31 The cross section for lepton pair photoproduction in a restricted phase space as a function of the leading lepton transverse momentum $P_{T}^{\ell_{1}}$ (left) and the invariant mass of the lepton pair $M_{\ell \ell}$ (right). The total error bar is shown, representing the statistical and

In the hadronic tau decay, due to the mass and charge of the tau lepton, most likely only one or three charged hadrons are produced. ${ }^{9}$ These decays modes are referred to as 1-prong

\footnotetext{
9 The branching ratio for tau decays into more than three charged hadrons is small (about $2 \%$ ).
}

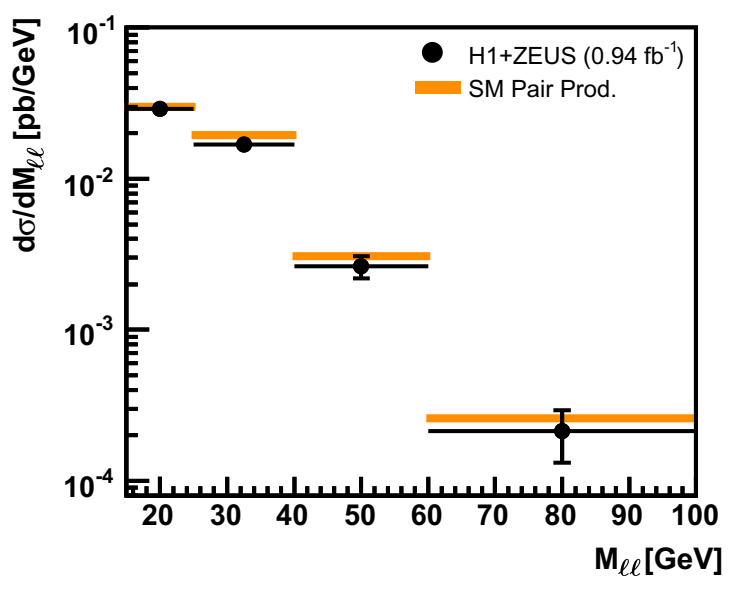

systematic uncertainties added in quadrature, which is dominated by the statistical. The bands represent the one standard deviation uncertainty in the SM prediction, dominated by the photon-photon process

(tau-decay branching ratio $49 \%$ ) and 3-prong (tau-decay branching ratio $14 \%$ ), respectively. The hadronic decay products of the tau lepton look like a collimated jet, featuring only one or three tracks, which are very close to each other. A $\tau$ jet can therefore be distinguished from QCD jets based on its shape and on the charge and multiplicity of its tracks. Neural 
networks and multivariate analysis techniques are typically employed to separate identified tau jet candidates from the large QCD background. One of the main differences between the analysis of tau-pair production and other di-lepton analyses is that a significant part of the tau-lepton momentum is carried away by the tau neutrino. This results in the taudecay products having a significantly lower $P_{T}$ compared to the original tau lepton.

The H1 [162] and ZEUS [174] Collaborations have both studied tau-pair production looking at the leptonic $(e \mu)$, semi-leptonic ( $e$ jet or $\mu$ jet) and hadronic (jet jet) decay modes. The tau-pair signal is modelled using the GRAPE event generator, which is also used to model the background expectation from other di-leptons. In order to reduce the significant SM background contribution from NC DIS and photoproduction events, the analyses are restricted to elastic or quasi-elastic tau-pair production, $e p \rightarrow e X \tau^{+} \tau^{-}$, where $X$ is the proton or a resonant state. This is effectively done by vetoing any additional objects in the final state beyond the tau-decay products and the scattered electron, which may also be present.

The main background contribution after applying the above elasticity requirements is due to exclusive diffractive events, both in DIS and photoproduction, which is modelled using the RAPGAP event generator. Non-diffractive DIS background is modelled by H1 (ZEUS) using RAPGAP (DJANGOH) and photoproduction is modelled by both experiments using PYTHIA.

The $\mathrm{H} 1$ analysis of tau-pair production is based on their HERA I data sample [162], corresponding to an integrated luminosity of $106 \mathrm{pb}^{-1}$. Electrons are selected with $E_{e}>$ $5 \mathrm{GeV}$ and $P_{T}^{e}>3 \mathrm{GeV}$, and muons are required to have $P_{T}^{\mu}>2 \mathrm{GeV}$. Both electrons and muons are reconstructed in the central region of the detector, $20^{\circ}<\theta_{\ell}<140^{\circ}$, and are required to be isolated from jets of at least one unit in angular distance $D=\sqrt{(\Delta \eta)^{2}+(\Delta \phi)^{2}}$. Jets composed of one or three tracks with $E_{T}^{\mathrm{jet}}>2 \mathrm{GeV}$ and in the region $20^{\circ}<\theta_{\text {jet }}<$ $120^{\circ}$ are reconstructed by a dedicated algorithm, which uses multiple neural networks to discriminate between tau jets and the QCD background [162].

In total, 30 tau-pair events are selected in the $\mathrm{H} 1$ analysis, compared to a SM prediction of $27.1 \pm 4.1$, of which $16.0 \pm 3.4$ are expected from $\gamma \gamma \rightarrow \tau^{+} \tau^{-}$events. The number of events collected in the different tau-pair topologies are summarised in Table 7. The distributions of the visible invariant mass, as well as the polar angle and transverse momentum of the identified tau candidates were also examined and show no deviation from the SM predictions. A tau-pair event in the final H1 sample is shown in Fig. 32, where one of the tau leptons decays into a muon and the other undergoes a 3-prong hadronic decay. In this case, the scattered electron is not detected in the event.
The ZEUS analysis of tau-pair production is based on their HERA II data sample [174], corresponding to an integrated luminosity of $334 \mathrm{pb}^{-1}$. Electrons are selected with $P_{T}^{e}>$ $2 \mathrm{GeV}$, in the polar angle region $17^{\circ}<\theta_{e}<160^{\circ}$, isolated from the rest other calorimetric energy deposits by a distance $D>0.8$. Muons are selected as tracks in the central detectors matched to segments in the muon chambers, with $P_{T}^{\mu}>$ $2 \mathrm{GeV}$ and in the polar angle region $34^{\circ}<\theta_{\mu}<157^{\circ}$. The muon track is required to be separated by a distance $D>$ 1.0 from any other track in the event. Tau jets with at least one track and $E_{T}^{\mathrm{jet}}>5 \mathrm{GeV}$ are reconstructed in the region $\left|\eta^{\text {jet }}\right|<2$. A multivariate discrimination technique [175] is used to discriminate tau jets from QCD jets.

A total of 25 events are observed in the data, compared to a SM expectation of $34.8_{-3.8}^{+3.9}$, of which $23.2_{-0.7}^{+0.7}$ are from taupair production. The events are classified into the different tau-decay topologies in Table 7. Figure 33 shows the visible invariant mass, $M_{\tau \tau}^{\text {visible }}$, calculated from the two tau candidates, and the scalar sum of their visible transverse momenta, $\sum P_{T, \tau \tau}^{\text {visible }}$. No event with a visible mass $M_{\tau \tau}^{\text {visible }}$ greater than $50 \mathrm{GeV}$ is observed in the data. The highest visible-mass candidate observed in the ZEUS data, found in the $e-\mu$ topology, has $M_{\tau \tau}^{\text {visible }}=49 \mathrm{GeV}$. The SM prediction describes the data well and no excess is observed in either the high mass or high $\sum P_{T, \tau \tau}^{\text {visible }}$ regions.

$\mathrm{H} 1$ performs a measurement of the cross section for the elastic production of $\tau^{+} \tau^{-}$pairs in the kinematic region $P_{T}^{\tau}>2 \mathrm{GeV}$ and $20^{\circ}<\theta_{\tau}<140^{\circ}$. The cross section is calculated according to Eq. (43), where the acceptance is calculated using GRAPE. The H1 measured visible cross section for elastic tau pair production $e p \rightarrow e p \tau^{+} \tau^{-}$integrated over the kinematic phase space defined above is

$\sigma_{\tau^{+} \tau^{-}}=13.6 \pm 4.4$ (stat.) \pm 3.7 (sys.) pb,

where the first uncertainty is statistical and the second systematic. The result is in good agreement with the SM expectation from GRAPE of $11.2 \pm 0.3 \mathrm{pb}$. A similar cross section measurement is performed by ZEUS in the kinematic region $P_{T}^{\tau}>5 \mathrm{GeV}$ and $17^{\circ}<\theta_{\tau}<160^{\circ}$. The visible measured cross section in the ZEUS phase space is

$\sigma_{\tau^{+} \tau^{-}}=3.3 \pm 1.3$ (stat.) \pm 2.0 (sys.) pb,

which is in reasonable agreement with the SM expectation $5.7 \pm 0.2 \mathrm{pb}$, as evaluated using GRAPE.

\subsection{Search for doubly charged Higgs production}

The production in $e p$ collisions of a single doubly charged Higgs boson $H^{ \pm \pm}$could be a source of events containing multiple high $P_{T}$ leptons at HERA [176]. Figure 34 shows the possible diagrams for single $H^{++}$production in $e^{+} p$ collisions at HERA. Following the observed excess of high- 
Table 7 Observed and predicted event yields for the different event topologies in the H1 and ZEUS di-tau analyses. The total MC expectation includes the sum of tau-pair production, NC DIS and photoproduction, as well as electron and muon pair production. The experimental systematic uncertainties are quoted on the total MC expectations

Tau-pair production at HERA

$\mathrm{H} 1$ analysis $\left(\mathcal{L}=106 \mathrm{pb}^{-1}\right)$

\begin{tabular}{lcrl}
\hline Tau decay & Data & Total SM & Tau-pair production $(\%)$ \\
\hline$e \mu$ & 7 & $2.9 \pm 0.4$ & 56 \\
$e$ jet & 2 & $6.3 \pm 0.9$ & 47 \\
$\mu$ jet & 10 & $7.0 \pm 1.3$ & 85 \\
jet jet & 11 & $11.0 \pm 2.0$ & 50 \\
Total & 30 & $27.1 \pm 4.1$ & 59 \\
\hline
\end{tabular}

ZEUS analysis $\left(\mathcal{L}=334 \mathrm{pb}^{-1}\right)$

\begin{tabular}{lcll}
\hline Tau decay & Data & Total SM & Tau-pair production \\
\hline$e \mu$ & 4 & $3.6_{-0.3}^{+1.3}$ & $3.0_{-0.2}^{+0.3}$ \\
$e$ jet & 7 & $8.8_{-0.8}^{+1.8}$ & $5.3_{-0.2}^{+0.3}$ \\
$\mu$ jet & 4 & $8.0_{-1.2}^{+2.2}$ & $5.9_{-0.5}^{+0.5}$ \\
jet jet & 10 & $14.4_{-3.5}^{+2.2}$ & $9.0_{-0.3}^{+0.4}$ \\
Total & 25 & $34.8_{-3.8}^{+3.9}$ & $23.2_{-0.7}^{+0.7}$ \\
\hline
\end{tabular}

mass events observed in multi-electron events in the $\mathrm{H} 1$ HERA data [168], these events were investigated in this context [177]. The compatibility of these events with a hypothetical doubly charged Higgs coupling to $e e$ is addressed and further searches for a $H^{ \pm \pm}$boson coupling to $e \mu$ and $e \tau$ are performed.

The $e e$ analysis is based on the multi-electron event selection described in Sect. 11 and [168], where a like-charge requirement [177] is added to the two highest electrons (positrons), which are assigned to the hypothetical $\mathrm{H}^{--}$ $\left(H^{++}\right)$. After the addition of the charge requirement, 3 events are observed in the data with $M_{e e}>65 \mathrm{GeV}$, in agreement with the SM expectation of $2.45 \pm 0.11$. Only one of the highmass events seen in the original multi-electron analysis [168] survives the additional selection requirements.

Events with one electron and one muon with minimal transverse momenta of $P_{T}^{e}>10 \mathrm{GeV}$ and $P_{T}^{\mu}>5 \mathrm{GeV}$ are selected to investigate the $H^{ \pm \pm} \rightarrow e \mu$ decay. Electrons are selected in the polar angle range $20^{\circ}<\theta_{e}<140^{\circ}$ to reduce the NC DIS background, and muons in the range $10^{\circ}<\theta_{\mu}<140^{\circ}$, which is more extended into the forward region to increase the efficiency for high $H^{ \pm \pm}$masses. The same charge criterion is applied to the $e \mu$ final states as used in the $e e$ search. For $M_{e \mu}>65 \mathrm{GeV}$ one event is observed, compared to a SM expectation of $4.17 \pm 0.44$.

The search for a $H^{++}$boson decaying into $e \tau$ is performed in three final states, depending on whether the tau-lepton decays into an electron, a muon or hadronically. ${ }^{10}$ Events are selected which contain either two electrons $(e e)$, or an electron and a muon $(e \mu)$, or an electron and a hadronic taujet $(e h)$. The two leptons, or the electron and the hadronic tau-jet (see Sect. 11.2) are required to be in the angular range $20^{\circ}<\theta<140^{\circ}$, and they have an angular separation of $D>2.5$ in pseudorapidity-azimuth. A minimum $P_{T}$ of 10 (5) $\mathrm{GeV}$ is required for the leading (second) lepton or hadronic tau-jet. A significant amount of missing transverse and longitudinal momentum is expected due to the neutrinos produced in the tau-lepton decay. Events in the $e e$ class are required to have a missing transverse momentum $P_{T}^{\text {miss }}>8 \mathrm{GeV}$. This is increased to $P_{T}^{\text {miss }}>11 \mathrm{GeV}$ for the $e h$ class and additional cuts are applied on the longitudinal balance of the event to reduce large NC DIS background [177]. Finally, events are rejected if the track associated with one of the Higgs decay product candidates has a negative charge, which is opposite to that of the incoming lepton beam. Following all $e \tau$ selection cuts, only one event is observed in the data (in the eh class), compared to a SM expectation of $2.1 \pm 0.5$.

Overall good agreement is observed with the SM predictions and no evidence for a doubly charged Higgs boson $H^{ \pm \pm}$is found in the data. Upper limits on the coupling $h_{e \ell}$ are derived at $95 \% \mathrm{CL}$ using a modified frequentist approach [178] taking into account systematic uncertainties and assuming that the $H^{ \pm \pm}$couples with $100 \%$ branching ratio to only $e e, e \mu$ or $e \tau$.

The $\mathrm{H} 1$ limits on $h_{e e}$ are not competitive to those set by the OPAL experiment [179]. The $e \mu(e \tau)$ analysis allows masses below $141 \mathrm{GeV}(112 \mathrm{GeV})$ to be ruled out at $95 \% \mathrm{CL}$ for a coupling $h_{e \mu}\left(h_{e \tau}\right)$ of electromagnetic strength $h_{e \ell}=0.3$, which extends beyond the limits from LEP [180-182]. More recent analyses from hadron colliders, which investigate $H^{ \pm \pm}$pair production and are independent of the coupling strength, have pushed these limits further, first beyond the $200 \mathrm{GeV}$ regime at the Tevatron (for example, an analysis of $e \mu$ final states from CDF, $M_{H^{ \pm \pm}}>210 \mathrm{GeV}$ [183]) and later at the LHC where the most stringent limits are from ATLAS $[184,185]$ and currently exclude masses up to $468 \mathrm{GeV}(400)$ in an analysis of $e \mu(e \tau)$ final states. The limits from the $\mathrm{H} 1$ remain unique in that they derived from a search for single $H^{ \pm \pm}$production.

\section{Events with isolated leptons and missing transverse momentum}

Events containing high-energy charged leptons (electron, muon or tau-leptons) together with missing transverse

\footnotetext{
10 The search for the $e \tau$ decay is only performed for using the larger $e^{+} p$ data set, so here only a $H^{++}$is considered.
} 

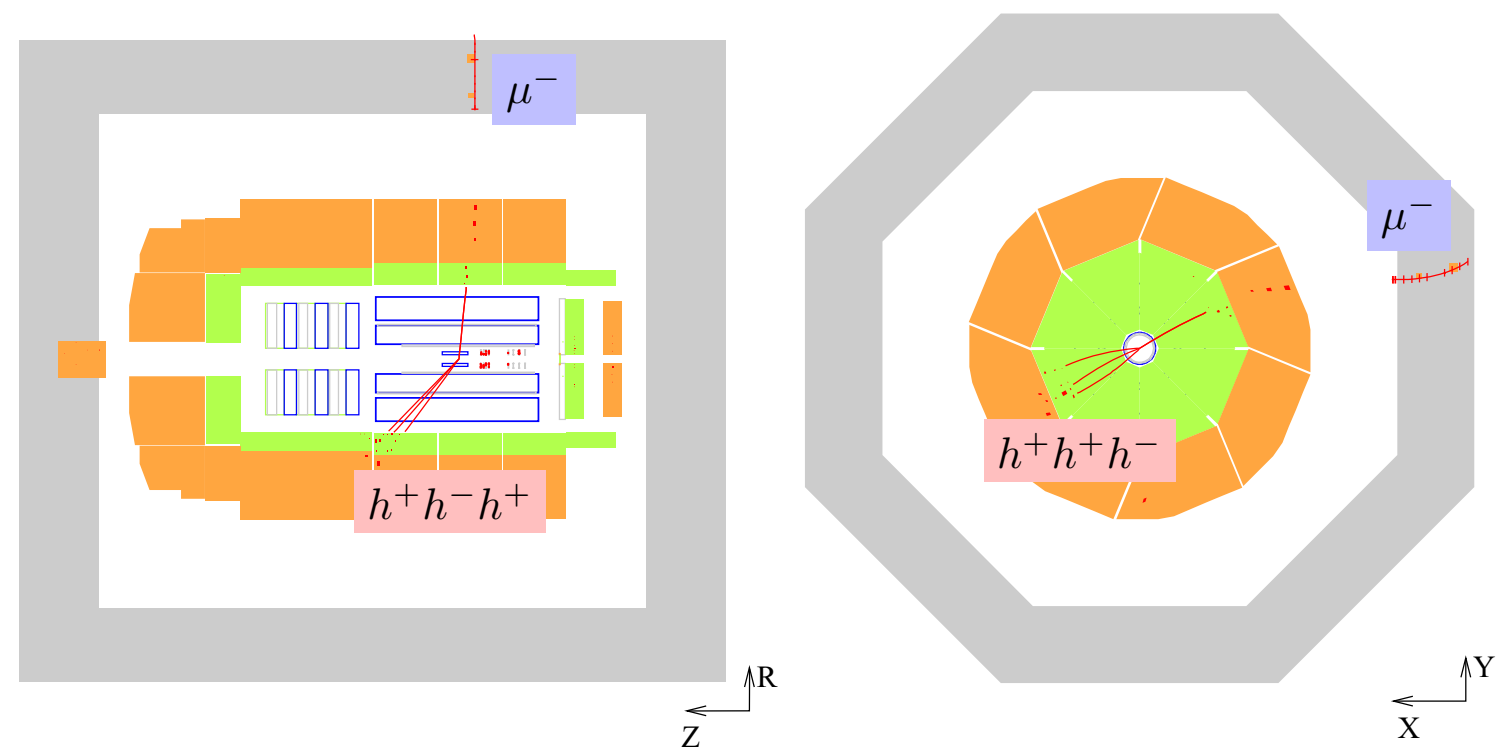

Fig. 32 A tau-pair event observed in the $\mathrm{H} 1$ detector, where one tau lepton decays leptonically to a muon and the other tau lepton decays to three charged hadrons, a so-called 3-prong decay

Fig. 33 Distribution of ZEUS tau-pair candidates as a function of a the visible invariant mass of the tau pair, $M_{\tau \tau}^{\text {visible }}$, and $\mathbf{b}$ the scalar sum of transverse momenta of the two tau candidates, $\sum p_{T, \tau \tau}^{\mathrm{visible}}$. The data (points) are compared with the predictions of the sum of the MC expectations. The tau-pair expectation is given by the hatched histogram. The shaded band represents the systematic uncertainty on the SM expectation

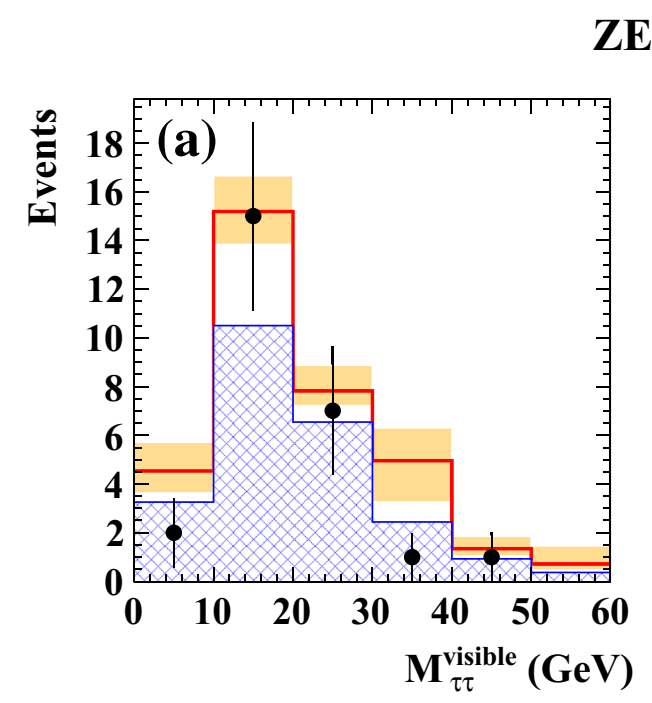

ZEUS

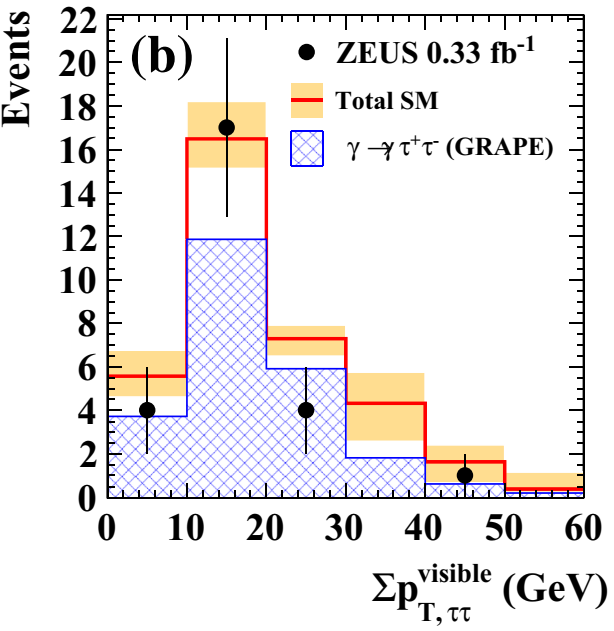

momentum produced in high-energy particle collisions are interesting as they may be a signature of BSM physics. When such an event containing an isolated muon was observed by $\mathrm{H} 1$ in the first $4 \mathrm{pb}^{-1}$ of $e^{+} p$ data, a detailed investigation into the potential physics source was performed [186]. After this initial $\mathrm{H} 1$ event was discovered during the visual scanning of high $Q^{2}$ events with large $P_{T}^{\text {miss }}$, which was routinely done during data taking for CC DIS analysis, such events would regularly appear throughout HERA I and II data taking.

In this analysis, processes are considered signal if they produce events containing a genuine isolated lepton and genuine missing transverse momentum in the final state. Within the SM, single $W$ boson production with subsequent leptonic decay $W \rightarrow \ell v$, as illustrated in Fig. 35a-c, is the main SM process at HERA that produces events with high- energy isolated leptons and missing transverse momentum. The inclusive hadronic state, which results primarily ${ }^{11}$ from the hadronisation of the struck quark $q^{\prime}$, is denoted by $X$. The SM prediction for $W$ production via $e p \rightarrow e W X$ is modelled by both $\mathrm{H} 1$ and ZEUS using the EPVEC event generator, which employs the full set of LO diagrams [101], including $W$ production via the $W W \gamma$ triple gauge boson coupling as illustrated in Fig. 35b. This prediction is corrected to NLO by applying a reweighting to the LO cross section dependent on the transverse momentum and rapidity of the $W$ boson [187-190]. The NLO corrections range from $30 \%$ at low $W$ transverse momentum (resolved photon

$\overline{11}$ In principle, the beam remnant may also contribute to the hadronic final state, although in the case of $W$ production this is typically low $P_{T}$ and continues down the beam pipe following the $e^{ \pm} p$ collision. 

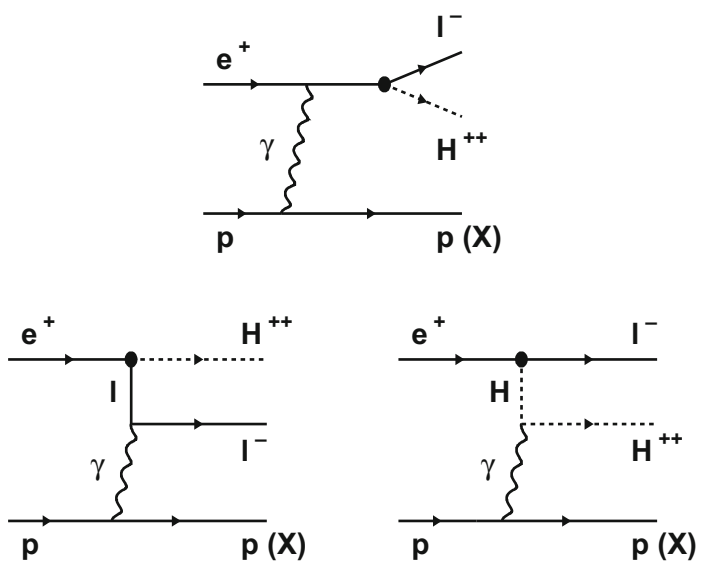

Fig. 34 Diagrams for the single production of a doubly charged Higgs boson in $e^{+} p$ collisions at HERA via a coupling $h_{e \ell}$

interactions) to around $10 \%$ at high $W$ transverse momentum (direct photon interactions).

Two further processes contribute to signal events. Firstly, the equivalent charged current $W$ production process ep $\rightarrow$ $v W X$, as illustrated in Fig. 35c, which is calculated at LO with EPVEC and contributes an additional $7 \%$ to the predicted signal cross section. Secondly, $Z^{0}$ production with subsequent decay to neutrinos, as illustrated in Fig. 35d, where the outgoing electron is the isolated lepton in the event and genuine missing transverse momentum is produced by the $Z^{0}$ decay neutrinos. This process, which is only relevant when the identified isolated lepton in the final state is an electron, is also calculated using EPVEC and found to contribute less than $3 \%$ to the predicted signal cross section.
Final states with isolated electrons or muons and missing transverse momentum, referred to as the electron channel and muon channel respectively, are discussed in the following. Analyses of events with isolated tau leptons and missing transverse momentum are covered in Sect. 12.2.

\subsection{Events with isolated electrons or muons}

Events with isolated electrons or muons and missing transverse momentum have been observed at HERA, and results from the analyses have been regularly published by both collaborations [191-194], culminating in the final results using the complete HERA data set $[195,196]$. The integrated luminosity in the $\mathrm{H} 1$ analysis corresponds to $474 \mathrm{pb}^{-1}$, of which $291 \mathrm{pb}^{-1}$ are from $e^{+} p$ collisions and $183 \mathrm{pb}^{-1}$ from $e^{-} p$ collisions. In the ZEUS analysis, the integrated luminosity corresponds to $504 \mathrm{pb}^{-1}$, of which $296 \mathrm{pb}^{-1}$ are from $e^{+} p$ collisions and $208 \mathrm{pb}^{-1}$ from $e^{-} p$ collisions.

The $\mathrm{H} 1$ and ZEUS event selections are very similar. Lepton candidates identified by $\mathrm{H} 1$ (ZEUS) are required to lie within the polar angle range $5^{\circ}<\theta_{\ell}<140^{\circ}\left(15^{\circ}<\theta_{\ell}<\right.$ $\left.120^{\circ}\right)$ and to have transverse momentum, $P_{T}^{\ell}>10 \mathrm{GeV}$. The lepton is also required to be isolated with respect to jets and other tracks in the event using the distances in $\eta-\phi$ space to the nearest jet $D_{\text {jet }}>1.0$ and nearest track $D_{\text {track }}>0.5$. A large transverse momentum imbalance $P_{T}^{\text {miss }}>12 \mathrm{GeV}$ is required and a cut on $P_{T}^{\text {calo }}>12 \mathrm{GeV}$ is also imposed to ensure a high trigger efficiency. As muons deposit little energy in the calorimeter $P_{T}^{\text {calo }} \simeq P_{T}^{X}$ in events with isolated muons, and therefore the $P_{T}^{\text {calo }}$ requirement effectively acts as a cut on the hadronic transverse momentum $P_{T}^{X}>12 \mathrm{GeV}$
Fig. 35 Diagrams of processes at HERA which produce an isolated lepton together with missing transverse momentum in the final state: Figure a $e p \rightarrow e W(\rightarrow \ell v) X$; figure b $W$ production via the $W W \gamma$ triple gauge boson coupling; figure $\mathbf{c} e p \rightarrow v W(\rightarrow \ell v) X$ figure $\mathbf{d} e p \rightarrow e Z(\rightarrow v \bar{v}) X$. The diagrams are shown for $e^{+} p$ collisions

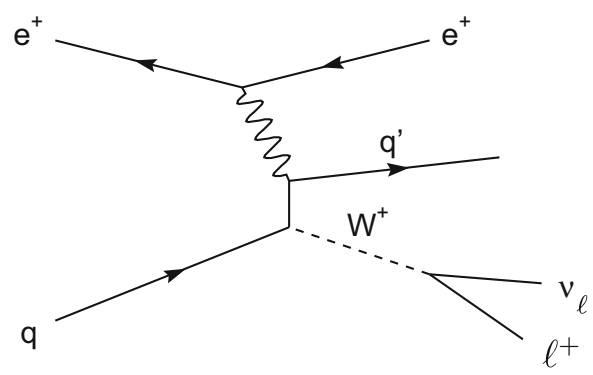

(a)

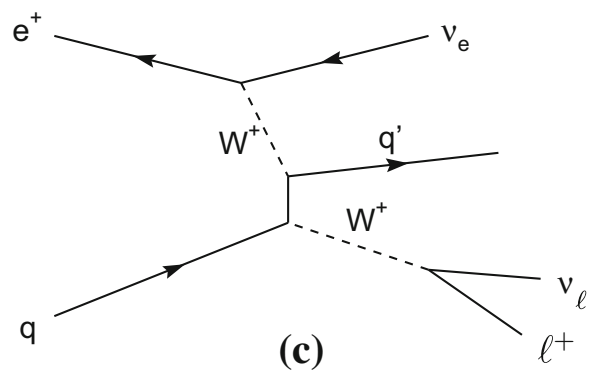

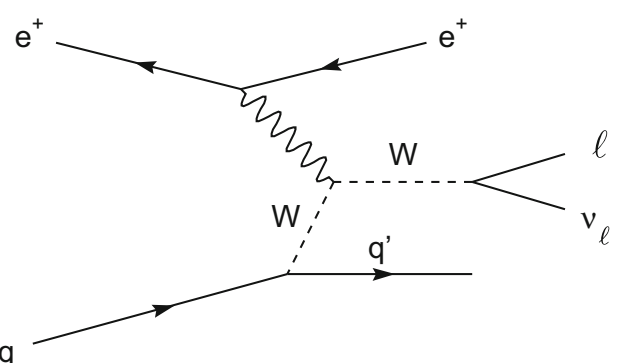

(b)

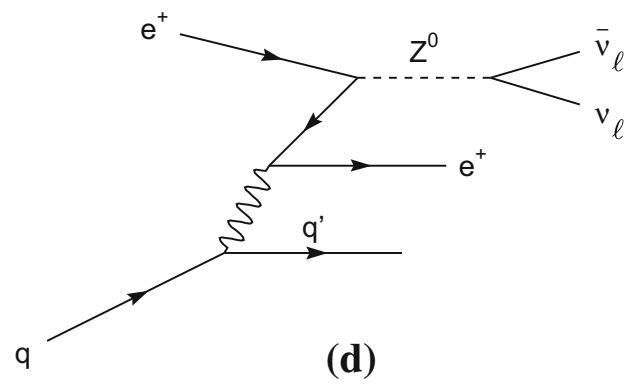


in the muon channel. To ensure that the two final states are exclusive, events in the electron channel must contain no isolated muons.

SM background processes contribute to the analysis mainly through misidentification or mismeasurement. NC DIS events $(e p \rightarrow e X)$, in which genuine isolated high $P_{T}$ electrons are produced, form a significant background in the electron channel search when fake $P_{T}^{\text {miss }}$ arises from mismeasurement. The NC DIS background is modelled in the H1 (ZEUS) analysis using the RAPGAP (DJANGOH) event generator. Charged current (CC) DIS events $\left(e p \rightarrow v_{e} X\right)$, in which there is real $P_{T}^{\text {miss }}$ due to the escaping neutrino, contribute to the background when fake isolated electrons or muons are observed and is modelled by both $\mathrm{H} 1$ and ZEUS using DJANGOH. Lepton pair production $\left(e p \rightarrow e \ell^{+} \ell^{-} X\right)$ contributes to the background via events where one lepton escapes detection and/or measurement errors cause apparent missing transverse momentum and is modelled by both $\mathrm{H} 1$ and ZEUS using the GRAPE event generator.

In order to reject the NC background contribution in the electron channel, further cuts are applied on the calorimetric energy imbalance, $V_{\mathrm{ap}} / V_{\mathrm{p}}$ [102], and the longitudinal momentum imbalance, $\delta_{\text {miss }}=2 E_{e}^{0}-\delta$, where $\delta$ is the total $E-P_{z}$ in the event as defined in Eq. (13) and $E_{e}^{0}$ is the electron beam energy. In the case of $\mathrm{H} 1$, the cut on $\delta_{\text {miss }}$ is only performed if the event contains exactly one electron, which has the same charge as the beam lepton. A cut on the difference in azimuthal angle between the lepton and the direction of the hadronic system, $\Delta \phi_{\ell-X}<160^{\circ}$, is used to reject NC DIS background, which has a back-to-back topology. Further background rejection in the electron channel is achieved using $\zeta_{e}^{2}=4 E_{e} E_{e}^{0} \cos ^{2} \theta_{e} / 2$, where $E_{e}$ is the energy of the final-state electron. ${ }^{12}$ Lepton pair background is removed from the muon channel by rejecting azimuthally balanced events using $V_{\mathrm{ap}} / V_{\mathrm{p}}$ and by requiring $\Delta \phi_{\mu-X}<170^{\circ}$, as well as by rejecting events with two or more isolated muons. Finally, the lepton-neutrino transverse mass:

$M_{T}^{\ell v}=\sqrt{\left(P_{T}^{\text {miss }}+P_{T}^{\ell}\right)^{2}-\left(\mathbf{p}_{T}^{\text {miss }}+\mathbf{p}_{T}^{\ell}\right)^{2}}$

is required to be larger than $10 \mathrm{GeV}$ in the $\mathrm{H} 1$ analysis to further reject NC (lepton pair) background in the electron (muon) channel. Full details of the event selections can be found in the individual publications $[195,196]$.

A total of 53 events are observed in the $\mathrm{H} 1$ analysis, in good agreement with the SM prediction of $54.1 \pm 7.4$, which is dominated by the expectation from signal processes of $40.4 \pm 6.3$ [195]. The data event yield is made up of 39

\footnotetext{
12 For $\mathrm{NC}$ events, where the scattered electron is identified as the isolated high transverse momentum electron, $\zeta_{e}^{2}$ is equal to the four momentum transfer squared $Q_{e}^{2}$, as calculated via electron method (see Sect. 6).
}

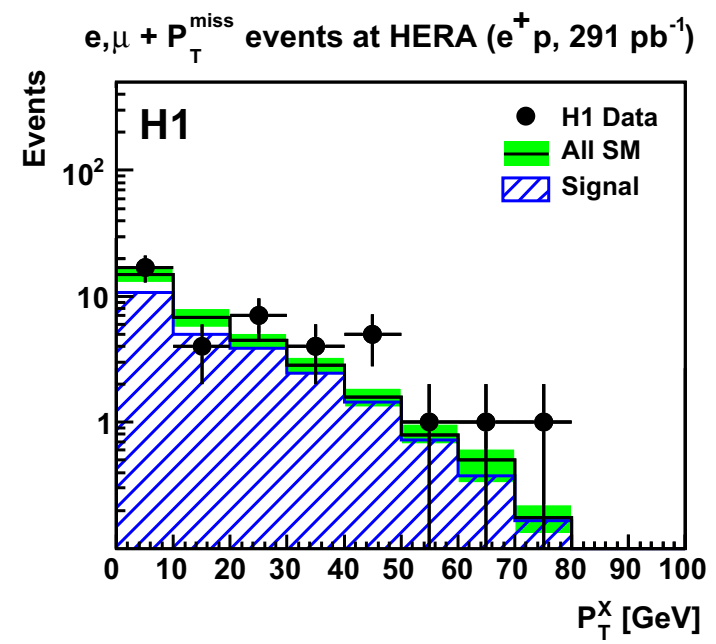

Fig. 36 The hadronic transverse momentum $P_{T}^{X}$ distribution for the electron and muon channels combined from the $\mathrm{H} 1$ analysis of the $e^{+} p$ data sample. The data (points) are compared to the SM expectation (open histogram). The signal component of the SM expectation, dominated by real $W$ production, is shown as the striped histogram. The total uncertainty on the SM expectation is shown as the shaded band

events in the electron channel and 14 events in the muon channel, compared to a SM prediction of $43.1 \pm 6.0$ and $11.0 \pm 1.8$ respectively. Fewer events are observed in the muon channel due to the $P_{T}^{X}$ cut, which is applied due to the $P_{T}^{\text {calo }}$ requirement of the trigger (see Sect. 5).

For large transverse momentum, $P_{T}^{X}>25 \mathrm{GeV}$, which is atypical of SM $W$ production, a total of 18 events are found in the data, compared to a SM prediction of $13.6 \pm 2.2$, of which 17 are found in $e^{+} p$ collisions compared to a SM prediction of $8.0 \pm 1.3$. The $P_{T}^{X}$ distribution for the combined $\mathrm{H} 1$ electron and muon channels is shown for the $e^{+} p$ data sample in Fig. 36. The observation of an excess of data events over the SM prediction, but only in the $e^{+} p$ data, is also a feature of earlier H1 publications [191,192].

A typical $W$ production event observed in the $\mathrm{H} 1$ analysis is displayed in Fig. 37 (top), featuring a single, isolated electron and large missing transverse momentum, which is clearly visible in the azimuthal plane. A further event from the $\mathrm{H} 1$ analysis is displayed in Fig. 37 (middle), this time featuring an isolated muon, large missing transverse momentum and a prominent hadronic final state with large $P_{T}^{X}$.

The results of the ZEUS analysis are summarised in Table 8, for the electron and muon channels combined. In the full phase space a total of 40 events are observed in the data broadly in agreement with the SM prediction [196]. For large hadronic transverse momentum, $P_{T}^{X}>25 \mathrm{GeV}, 11$ events are found in the data, compared to a SM prediction of $12.9 \pm 1.7$. Unlike the $\mathrm{H} 1$ analysis, no excess is observed in this region, where for the ZEUS $e^{+} p$ data 6 events are seen compared to a SM prediction of $7.4 \pm 1.0$. 

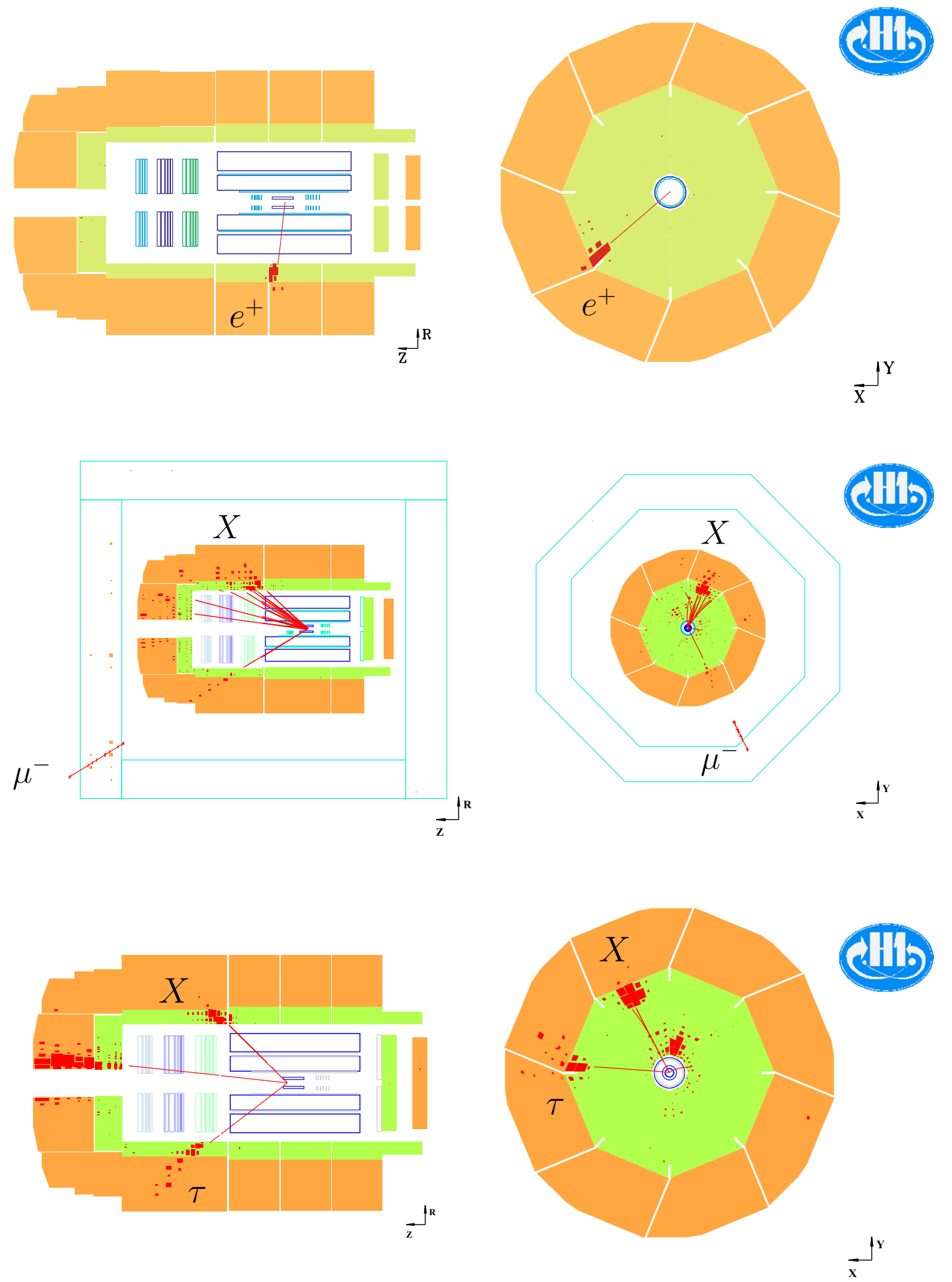

Fig. 37 Event displays in the $\mathrm{H} 1$ search for events with and isolated lepton and missing transverse momentum. Top an electron event with missing transverse momentum and no visible hadronic final state, typical of low $P_{T}$ single $W$ production. Middle an isolated muon event, with

missing transverse momentum and a prominent hadronic final state. Bottom an event with an isolated tau lepton candidate, missing transverse momentum and a prominent hadronic final state 
Table 8 ZEUS search for events with an isolated electron or muon and missing transverse momentum at HERA. The number of observed events is compared to the SM prediction. The fraction of the SM expec- tation arising from $W$ production is also given. The quoted errors contain statistical and systematic uncertainties added in quadrature

\begin{tabular}{|c|c|c|c|}
\hline \multicolumn{4}{|c|}{ Search for events with an isolated electron or muon and missing transverse momentum at HERA } \\
\hline \multicolumn{4}{|l|}{ ZEUS analysis } \\
\hline & Data & Total SM & $W$ production $(\%)$ \\
\hline \multicolumn{4}{|c|}{$e^{+} p$ collisions $\left(\mathcal{L}=296 \mathrm{pb}^{-1}\right)$} \\
\hline$P_{T}^{X}<12 \mathrm{GeV}$ & 7 & $12.6 \pm 1.7$ & 68 \\
\hline $12<P_{T}^{X}<25 \mathrm{GeV}$ & 7 & $6.2 \pm 0.9$ & 75 \\
\hline$P_{T}^{X}>25 \mathrm{GeV}$ & 6 & $7.4 \pm 1.0$ & 79 \\
\hline \multicolumn{4}{|c|}{$e^{-} p$ collisions $\left(\mathcal{L}=208 \mathrm{pb}^{-1}\right)$} \\
\hline$P_{T}^{X}<12 \mathrm{GeV}$ & 9 & $11.3 \pm 1.5$ & 54 \\
\hline $12<P_{T}^{X}<25 \mathrm{GeV}$ & 6 & $5.1 \pm 0.7$ & 67 \\
\hline$P_{T}^{X}>25 \mathrm{GeV}$ & 5 & $5.5 \pm 0.8$ & 75 \\
\hline \multicolumn{4}{|c|}{$e^{ \pm} p$ collisions $\left(\mathcal{L}=506 \mathrm{pb}^{-1}\right)$} \\
\hline$P_{T}^{X}<12 \mathrm{GeV}$ & 16 & $23.9 \pm 3.1$ & 61 \\
\hline $12<P_{T}^{X}<25 \mathrm{GeV}$ & 13 & $11.2 \pm 1.5$ & 71 \\
\hline$P_{T}^{X}>25 \mathrm{GeV}$ & 11 & $12.9 \pm 1.7$ & 77 \\
\hline
\end{tabular}

A combination of the H1 and ZEUS isolated lepton and missing transverse momentum analyses has also been performed, using the full HERA data sample corresponding to an integrated luminosity of $0.98 \mathrm{fb}^{-1}$ comprising $0.39 \mathrm{fb}^{-1}$ of $e^{-} p$ collisions and $0.59 \mathrm{fb}^{-1}$ of $e^{+} p$ collisions. A study of the selection efficiency for signal processes found the $\mathrm{H} 1$ and ZEUS analyses to be compatible in the kinematic region where they are directly comparable [197]. Nevertheless, in order to coherently combine the results from the two experiments, a common phase space is established [198]. The lepton polar angle acceptance is the main difference in the common phase space with respect to the $\mathrm{H} 1$ analysis, where the more restricted range of the ZEUS analysis is used, $15^{\circ}<$ $\theta_{\ell}<120^{\circ}$. Additionally, the more restrictive cuts on $\delta_{\text {miss }}$ and $V_{\text {ap }} / V_{\mathrm{p}}$ are taken from the ZEUS analysis [196]. The minimum lepton-neutrino transverse mass and electron multiplicity requirements are taken from the H1 analysis [195].

The results of the combined $\mathrm{H} 1$ and ZEUS analysis are summarised in Table 9. The signal contribution, mainly from real $W$ production, is seen to dominate the total SM expectation in all data samples. A total of 81 events are observed in the data, compared to a SM expectation of $87.8 \pm 11.0$. At large hadronic transverse momentum $P_{T}^{X}>25 \mathrm{GeV}$ a total of 29 events are observed in the $e^{ \pm} p$ data compared to a SM prediction of $24.0 \pm 3.2$. In the $e^{+} p$ data alone, 23 events are observed with $P_{T}^{X}>25 \mathrm{GeV}$ compared to a SM prediction of $14.0 \pm 1.9$. Seventeen of these data events are the same events observed in the standard H1 analysis [195], but now compared to a lower SM expectation of $6.7 \pm 0.9$ in the more restricted common phase space. Whilst this intriguing excess in the $e^{+} p$ analysis remains in the common phase space, it also remains a feature only seen in the $\mathrm{H} 1$ data.
Figure 38 shows a variety of kinematic distributions of the H1 and ZEUS combined analysis for the complete $e^{ \pm} p$ HERA I+II data, for the electron and muon channels together. The shape and normalisation of the distributions are well described within the uncertainties. The distribution of events in $M_{T}^{l v}$ is compatible with the Jacobian peak expected from $W$ production. Similarly, the observed $P_{T}^{X}$ spectrum is compatible with that expected from $W$ production, peaking at low values of hadronic transverse momentum.

A measurement of the visible cross section for the isolated lepton and missing transverse momentum topology in $e^{ \pm} p$ collisions is performed by $\mathrm{H} 1$ using the electron and muon channels in the phase space $5^{\circ}<\theta_{\ell}<140^{\circ}, P_{T}^{\ell}>10 \mathrm{GeV}$, $P_{T}^{\text {miss }}>12 \mathrm{GeV}$ and $D_{\text {jet }}>1.0$ at a centre of mass energy ${ }^{13}$ of $\sqrt{s}=317 \mathrm{GeV}$. The cross section is calculated using Eq. (43), where the EPVEC generator is used to calculate the acceptance, $\mathcal{A}$, which is predicted to be about the same for $e^{+} p$ and $e^{-} p$ collisions. The total visible cross section for events with an isolated lepton and missing transverse momentum is measured by $\mathrm{H} 1$ as:

$\sigma_{\ell+P_{T}^{\text {miss }}}=0.23 \pm 0.05$ (stat.) \pm 0.04 (sys.) pb,

where the first error is statistical and the second systematic, in agreement with the SM NLO value of $0.25 \pm 0.04 \mathrm{pb}$ from EPVEC.

The single- $W$ boson production cross section is measured by $\mathrm{H} 1$ and ZEUS, individually and in the combined analysis described above. The branching ratio corresponding to the leptonic $W$ boson decay to any final state with an electron

\footnotetext{
$\overline{13}$ Assuming a linear dependence of the cross section on the protonbeam energy.
} 
Table 9 Combined H1 and ZEUS search for events with an isolated electron or muon and missing transverse momentum at HERA. The results are shown for the full selected sample and for the subsample with hadronic transverse momentum $P_{T}^{X}>25 \mathrm{GeV}$. The number of observed events is compared to the SM prediction. The SM signal (dominated by single- $W$ production) and the total background contribution (mainly $\mathrm{NC}$ and CC DIS, together with lepton-pair production) are also shown. The quoted uncertainties contain statistical and systematic uncertainties added in quadrature

Search for events with an isolated electron or muon and missing transverse momentum at HERA

Combined $\mathrm{H} 1$ and ZEUS analysis

\begin{tabular}{|c|c|c|c|c|c|}
\hline \multicolumn{2}{|l|}{ Channel } & \multirow[t]{2}{*}{ Data } & \multirow[t]{2}{*}{ Total SM } & \multirow[t]{2}{*}{ SM signal } & \multirow[t]{2}{*}{ Other SM } \\
\hline \multicolumn{2}{|c|}{$e^{+} p$ collisions $\left(\mathcal{L}=0.59 \mathrm{fb}^{-1}\right)$} & & & & \\
\hline \multirow[t]{2}{*}{ Electron } & Total & 37 & $38.6 \pm 4.7$ & $28.9 \pm 4.4$ & $9.7 \pm 1.4$ \\
\hline & $P_{T}^{X}>25 \mathrm{GeV}$ & 12 & $7.4 \pm 1.0$ & $6.0 \pm 0.9$ & $1.5 \pm 0.3$ \\
\hline \multirow[t]{2}{*}{ Muon } & Total & 16 & $11.2 \pm 1.6$ & $9.9 \pm 1.6$ & $1.3 \pm 0.3$ \\
\hline & $P_{T}^{X}>25 \mathrm{GeV}$ & 11 & $6.6 \pm 1.0$ & $5.9 \pm 0.9$ & $0.8 \pm 0.2$ \\
\hline \multirow[t]{2}{*}{ Combined } & Total & 53 & $49.8 \pm 6.2$ & $38.8 \pm 5.9$ & $11.1 \pm 1.5$ \\
\hline & $P_{T}^{X}>25 \mathrm{GeV}$ & 23 & $14.0 \pm 1.9$ & $11.8 \pm 1.9$ & $2.2 \pm 0.4$ \\
\hline \multicolumn{6}{|c|}{$e^{-} p$ collisions $\left(\mathcal{L}=0.39 \mathrm{fb}^{-1}\right)$} \\
\hline \multirow[t]{2}{*}{ Electron } & Total & 24 & $30.6 \pm 3.6$ & $19.4 \pm 3.0$ & $11.2 \pm 1.9$ \\
\hline & $P_{T}^{X}>25 \mathrm{GeV}$ & 4 & $5.6 \pm 0.8$ & $4.0 \pm 0.6$ & $1.6 \pm 0.4$ \\
\hline \multirow[t]{2}{*}{ Muon } & Total & 4 & $7.4 \pm 1.1$ & $6.6 \pm 1.0$ & $0.9 \pm 0.3$ \\
\hline & $P_{T}^{X}>25 \mathrm{GeV}$ & 2 & $4.3 \pm 0.7$ & $3.9 \pm 0.6$ & $0.4 \pm 0.2$ \\
\hline \multirow[t]{2}{*}{ Combined } & Total & 28 & $38.0 \pm 3.4$ & $26.0 \pm 3.4$ & $12.0 \pm 2.0$ \\
\hline & $P_{T}^{X}>25 \mathrm{GeV}$ & 6 & $10.0 \pm 1.3$ & $7.9 \pm 1.2$ & $2.1 \pm 0.5$ \\
\hline \multicolumn{6}{|c|}{$e^{ \pm} p$ collisions $\left(\mathcal{L}=0.98 \mathrm{fb}^{-1}\right)$} \\
\hline \multirow[t]{2}{*}{ Electron } & Total & 61 & $69.2 \pm 8.2$ & $48.3 \pm 7.4$ & $20.9 \pm 3.2$ \\
\hline & $P_{T}^{X}>25 \mathrm{GeV}$ & 16 & $13.0 \pm 1.7$ & $10.0 \pm 1.6$ & $3.1 \pm 0.7$ \\
\hline \multirow[t]{2}{*}{ Muon } & Total & 20 & $18.6 \pm 2.7$ & $16.4 \pm 2.6$ & $2.2 \pm 0.5$ \\
\hline & $P_{T}^{X}>25 \mathrm{GeV}$ & 13 & $11.0 \pm 1.6$ & $9.8 \pm 1.6$ & $1.2 \pm 0.3$ \\
\hline \multirow[t]{2}{*}{ Combined } & Total & 81 & $87.8 \pm 11.0$ & $64.7 \pm 9.9$ & $23.1 \pm 3.3$ \\
\hline & $P_{T}^{X}>25 \mathrm{GeV}$ & 29 & $24.0 \pm 3.2$ & $19.7 \pm 3.1$ & $4.3 \pm 0.8$ \\
\hline
\end{tabular}

or muon, including the contribution from leptonic tau decay, is also included in the calculation. This cross section is also calculated using Eq. (43), where $\mathcal{A}$ is again calculated using EPVEC but is now defined with respect to the full phase space and the contribution from $Z^{0}$ production illustrated in Fig. 35d is considered as background. The acceptances for the two experiments are found to be similar in each $P_{T}^{X}$ bin and vary between 27 and $37 \%$ in the electron channel and between 18 and $38 \%$ in the muon channel [198].

The combined H1-ZEUS single- $W$ production cross section, evaluated using a weighted mean of the values measured by the two collaborations in the common phase space, is measured as

$\sigma_{W}=1.06 \pm 0.16$ (stat.) \pm 0.07 (sys.) pb,

where the first uncertainty is statistical and the second systematic. The measurement agrees well with the NLO SM prediction of $1.26 \pm 0.19 \mathrm{pb}$ from EPVEC and the measurements by $\mathrm{H} 1$ and ZEUS in their individual publications [195,196]. The single- $W$ boson production cross section is also measured differentially as a function of $P_{T}^{X}$, the results of which are displayed in Fig. 39, and is also found to be in agreement with the SM prediction.

Some additional investigations into $W$ bosons are performed by H1 using the analysis of their full data set [195]. The production of single $W$ bosons at HERA is sensitive to anomalous triple gauge boson couplings [199] via the process illustrated in Fig. 35b, which can be parametrised using two free coupling parameters, $\kappa$ and $\lambda$ [200]. In the SM $\kappa=1$ and $\lambda=0$ at tree level and in the following $\Delta \kappa \equiv \kappa-1$ is used, such that any non-zero value for $\Delta \kappa$ or $\lambda$ represents a deviation from the SM. The hadronic transverse momentum spectrum of $W$ events is expected to be sensitive to anomalous values of $\Delta \kappa$ and $\lambda$ [199], and a likelihood analysis on the measured $P_{T}^{X}$ distribution is performed using a Bayesian approach and employing Poisson statistics [195]. This is done for $\Delta \kappa$ and $\lambda$ separately, keeping the other parameter fixed to its SM value. The following limits are derived at $95 \% \mathrm{CL}$ :

$-0.7<\Delta \kappa<1.4$

$-2.5<\lambda<2.5$. 
Fig. 38 Distributions of kinematic variables of events with an isolated electron or muon and missing transverse momentum in the full HERA $e^{ \pm} p$ data. Shown are the polar angle of the lepton $\theta_{\ell}$ (a), the difference in the azimuthal angle of the lepton and the hadronic systems $\Delta \phi_{\ell-X}(\mathbf{b})$, the lepton-neutrino transverse mass $M_{T}^{\ell v}(\mathbf{c})$, the hadronic transverse momentum $P_{T}^{X}(\mathbf{d})$, the missing transverse momentum $P_{T}^{\text {miss }}(\mathbf{e})$ and the transverse momentum of the lepton $P_{T}^{\ell}(\mathbf{f})$. The data (points) are compared to the SM expectation (open histogram).

The signal component of the SM expectation, dominated by single- $W$ production, is shown as the striped histogram. The total uncertainty on the SM expectation is shown as the shaded band
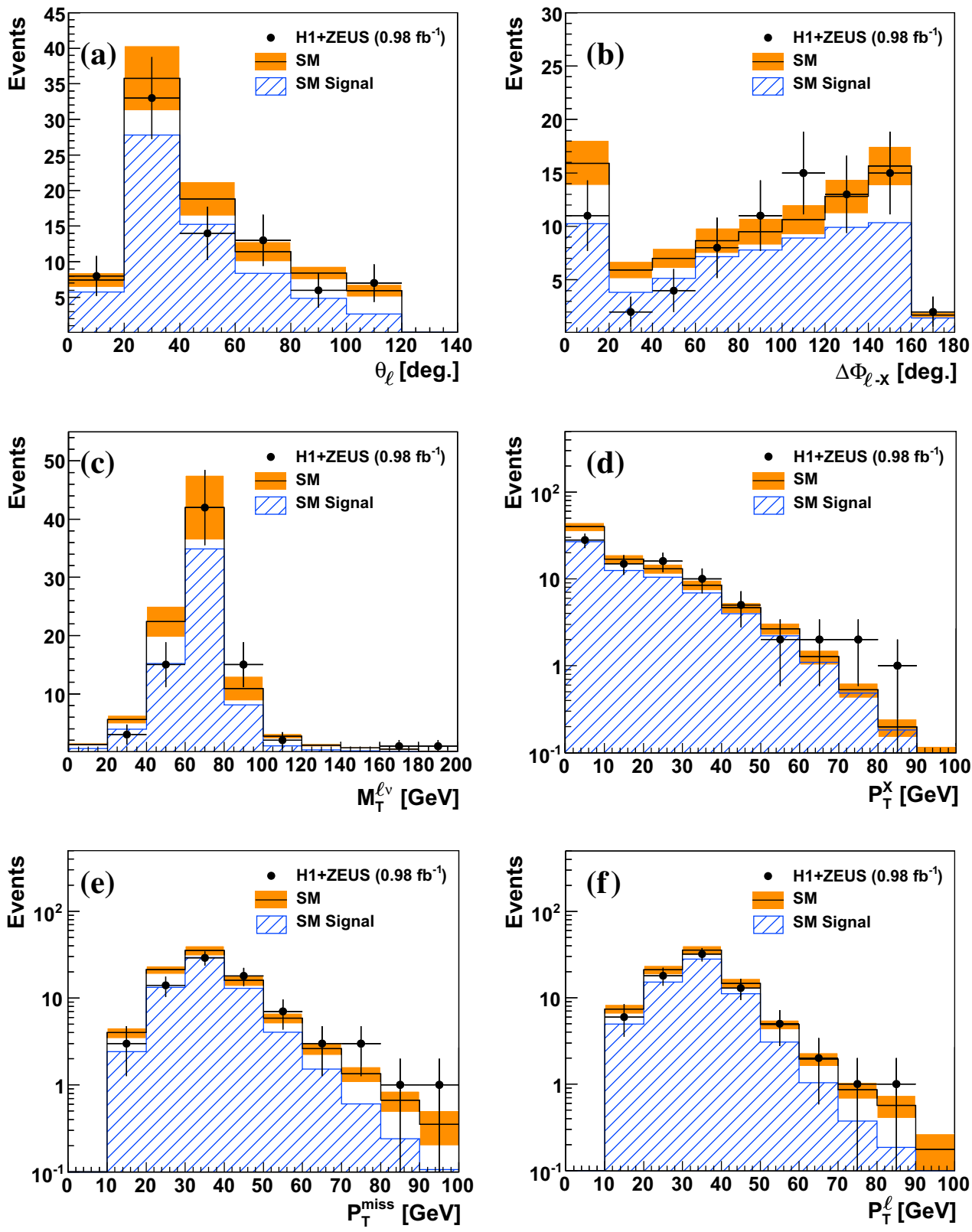

in good agreement with the SM prediction and since the value of $\Delta \kappa=-1$ is excluded at $95 \% \mathrm{CL}$, the results obtained explicitly demonstrate the presence of a magnetic coupling of the photon to the $W$ boson, in addition to the coupling to the electric charge of the $W$ boson. The most stringent limits on these couplings were obtained by the LEP experiments in single- $\gamma$, single- $W$ and $W$ pair production [201-204].

$\mathrm{H} 1$ also performs a measurement of the $W$ boson polarisation fractions at HERA by examining the $\cos \theta^{*}$ distribution in the decay $W \rightarrow e / \mu+\nu$, where $\theta^{*}$ is defined as the angle between the $W$ boson momentum in the lab frame and the charged decay lepton in the $W$ boson rest frame. The $\cos \theta^{*}$ distributions for $W^{+}$bosons are given by [200]:

$$
\begin{aligned}
\frac{d N}{d \cos \theta^{*}} & \propto\left(1-F_{-}-F_{0}\right) \cdot \frac{3}{8}\left(1+\cos \theta^{*}\right)^{2} \\
& +F_{0} \cdot \frac{3}{4}\left(1-\cos ^{2} \theta^{*}\right) \\
& +F_{-} \cdot \frac{3}{8}\left(1-\cos \theta^{*}\right)^{2},
\end{aligned}
$$

where the left-handed $F_{-}$, longitudinal $F_{0}$ and right-handed $F_{+}$polarisation fractions are constrained by the relation $F_{+} \equiv 1-F_{-}-F_{0}$. Beginning with the $\mathrm{H} 1$ isolated electron or muon and missing transverse momentum event sample, the additional requirement of a reliable charge measurement is added, so that the resulting charge misidentification is well below $1 \%$ [195]. The final event sample consists of 21 electron events and 9 muon events and a SM prediction with a $W$ 


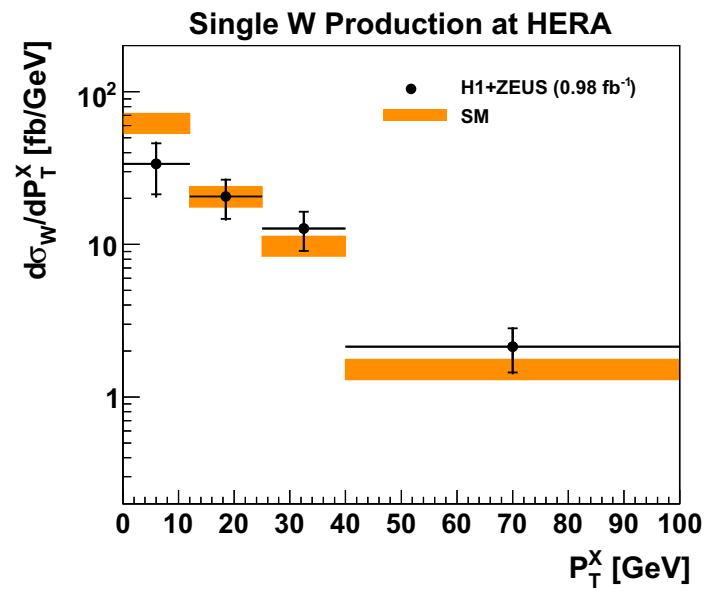

Fig. 39 The single- $W$ production cross section as a function of the hadronic transverse momentum, $P_{T}^{X}$, measured using the combined $\mathrm{H} 1$ and ZEUS data at a centre of mass energy of $\sqrt{s}=317 \mathrm{GeV}$. The inner error bar represents the statistical error and the outer error bar indicates the statistical and systematic uncertainties added in quadrature. The shaded band represents the uncertainty on the SM prediction

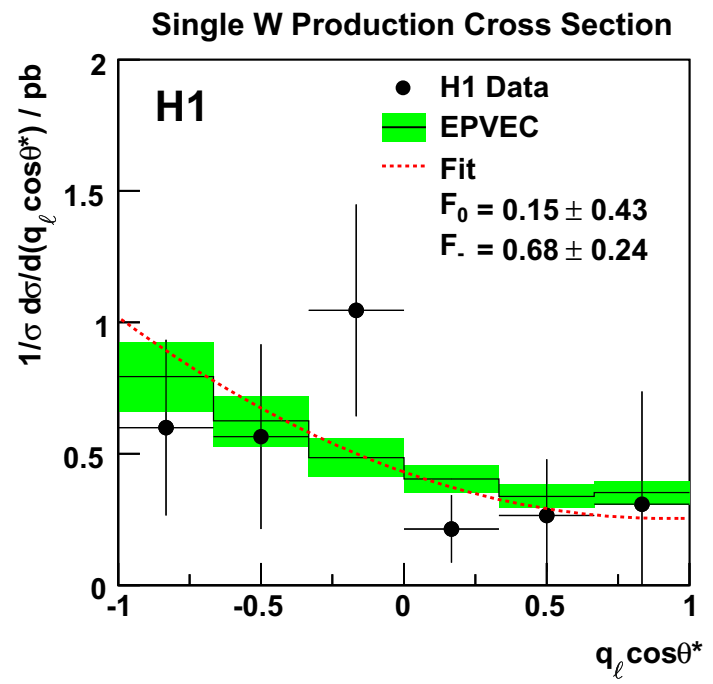

Fig. 40 The $\mathrm{H} 1$ measured normalised differential cross section $1 / \sigma d \sigma / d\left(q_{\ell} \cdot \cos \theta^{*}\right)$ (points) as a function of $q_{\ell} \cdot \cos \theta^{*}$ for on-shell $W$ bosons. The EPVEC prediction is also shown (open histogram) with a $15 \%$ theoretical uncertainty shown by the band. The result of the simultaneous fit of the $W$ polarisation fractions is shown as the dashed histogram

production purity of $76 \%$. To allow the combination of the different $W$ boson charges, the value of $\cos \theta^{*}$ is multiplied by the sign of the lepton charge $q_{\ell}= \pm 1$ and the measured distribution, corrected for acceptance and detector effects.

The normalised differential cross section as a function of $q_{\ell} \cdot \cos \theta^{*}$ is shown in Fig. 40, compared to the SM prediction. The cross section fit to the model defined in Eq. (45) is also shown. The optimal values for the $W$ boson polarisation fractions $F_{-}$and $F_{0}$ are simultaneously extracted from the fit as $0.68 \pm 0.24$ and $0.15 \pm 0.43$, respectively, in good agreement with the SM values 0.62 and 0.17 as predicted by EPVEC.

\subsection{Events with isolated tau-leptons}

The search for isolated tau leptons complements the analysis of the electron and muon channels described in Sect. 12.1. This analysis has previously been performed by $\mathrm{H} 1$ and ZEUS using their HERA I data sets, where the tau is identified by its hadronic decay $[162,205]$. The H1 Collaboration have updated their search to include their full HERA data set, corresponding to an integrated luminosity of $474 \mathrm{pb}^{-1}$ [195].

The selection of $\mathrm{H} 1$ events with isolated tau leptons and missing transverse momentum is based on the HERA-I analysis [162]. Only hadronic decays with one charged hadron (one-prong) are considered. Tau decays to electrons and muons enter the electron and muon channels described in Sect. 12.1.

A tau identification algorithm selects narrow, low multiplicity jets typical for hadronic tau decays, with transverse momentum $P_{T}^{\text {jet }}>7 \mathrm{GeV}$ in the central region $20^{\circ}<\theta_{\text {jet }}<$ $120^{\circ}$ of the detector. Narrow jets are selected by requiring $R_{\text {jet }}<0.12$ (see Eq. (42)). At least one track measured in the central tracking detector with transverse momentum $P_{T}^{\text {track }}>5 \mathrm{GeV}$ is required to be associated to the jet. In the final selection, the tau jet is required to be isolated from electrons, muons and other jets in the event by requiring a minimum angular separation in $\eta-\phi$ space of $D>1.0$.

To ensure the presence of neutrinos in the event, large $P_{T}^{\text {miss }}>12 \mathrm{GeV}$ and $P_{T}^{\text {calo }}>12 \mathrm{GeV}$, a significant azimuthal imbalance $\delta_{\text {miss }}=2 E_{e}^{0}-\delta>5 \mathrm{GeV}$ and low $V_{\mathrm{ap}} / V_{\mathrm{p}}<0.5$ are required. The event has also to exhibit large inclusive hadronic transverse momentum $P_{T}^{X}>12 \mathrm{GeV}$. Some degree of acoplanarity between the tau jet and the remaining hadronic system $X^{\prime}$ in the transverse plane $\Delta \phi_{\tau-X^{\prime}}<170^{\circ}$ is required to suppress events with back-to-back topologies, primarily NC events and photoproduction events with jets.

The results of the search in the tau channel are summarised in Table 10. In the final event sample, 18 events are selected, compared to a SM expectation of $23.2 \pm 3.8$. Unlike in the search for events with an isolated electron or muon and missing transverse momentum, the SM expectation is dominated by CC DIS background processes, and the signal contribution is only $11 \%$. Distributions of the events in the final sample are shown in Fig. 41. Most of the events are observed at very low $P_{T}^{X^{\prime}}$. At $P_{T}^{X^{\prime}}>25 \mathrm{GeV}$ one event is observed in the data, compared to a SM expectation of $1.5 \pm 0.2$. In this region the contribution of single- $W$ boson production to the SM expectation is about $38 \%$. The selected data event, shown in Fig. 37 (bottom), is observed in $e^{-} p$ collisions and exhibits $P_{T}^{\tau}=14.3 \pm 1.2 \mathrm{GeV}, P_{T}^{X^{\prime}}=62 \pm 5 \mathrm{GeV}$ and $P_{T}^{\text {miss }}=68 \pm 6 \mathrm{GeV}$. 
Table 10 Summary of the $\mathrm{H} 1$ results of the search for events with tau leptons and missing transverse momentum for the $e^{+} p$ data, $e^{-} p$ data and the full $\mathrm{H} 1$ data set. The results are shown for the full selected sample and for the subsample at $P_{T}^{X}>25 \mathrm{GeV}$. The number of observed events is compared to the SM prediction. The SM signal $\left(W \rightarrow \tau \nu_{\tau}\right)$ and the background contributions are also shown. The quoted uncertainties contain statistical and systematic uncertainties added in quadrature

$\mathrm{H} 1$ search for events with an isolated tau-lepton and missing transverse momentum at HERA

\begin{tabular}{|c|c|c|c|c|}
\hline & Data & Total SM & SM signal & Other SM \\
\hline \multicolumn{5}{|c|}{$e^{+} p$ collisions $\left(\mathcal{L}=291 \mathrm{pb}^{-1}\right)$} \\
\hline Total & 9 & $12.3 \pm 2.0$ & $1.66 \pm 0.25$ & $10.6 \pm 1.8$ \\
\hline$P_{T}^{X}>25 \mathrm{GeV}$ & 0 & $0.82 \pm 0.12$ & $0.38 \pm 0.06$ & $0.44 \pm 0.06$ \\
\hline \multicolumn{5}{|c|}{$e^{-} p$ collisions $\left(\mathcal{L}=183 \mathrm{pb}^{-1}\right)$} \\
\hline Total & 9 & $11.0 \pm 1.9$ & $1.00 \pm 0.15$ & $10.0 \pm 1.8$ \\
\hline$P_{T}^{X}>25 \mathrm{GeV}$ & 1 & $0.68 \pm 0.11$ & $0.21 \pm 0.03$ & $0.47 \pm 0.07$ \\
\hline \multicolumn{5}{|c|}{$e^{ \pm} p$ collisions $\left(\mathcal{L}=474 \mathrm{pb}^{-1}\right)$} \\
\hline Total & 18 & $23.2 \pm 3.8$ & $2.66 \pm 0.40$ & $20.6 \pm 3.4$ \\
\hline$P_{T}^{X}>25 \mathrm{GeV}$ & 1 & $1.50 \pm 0.21$ & $0.59 \pm 0.09$ & $0.91 \pm 0.12$ \\
\hline
\end{tabular}
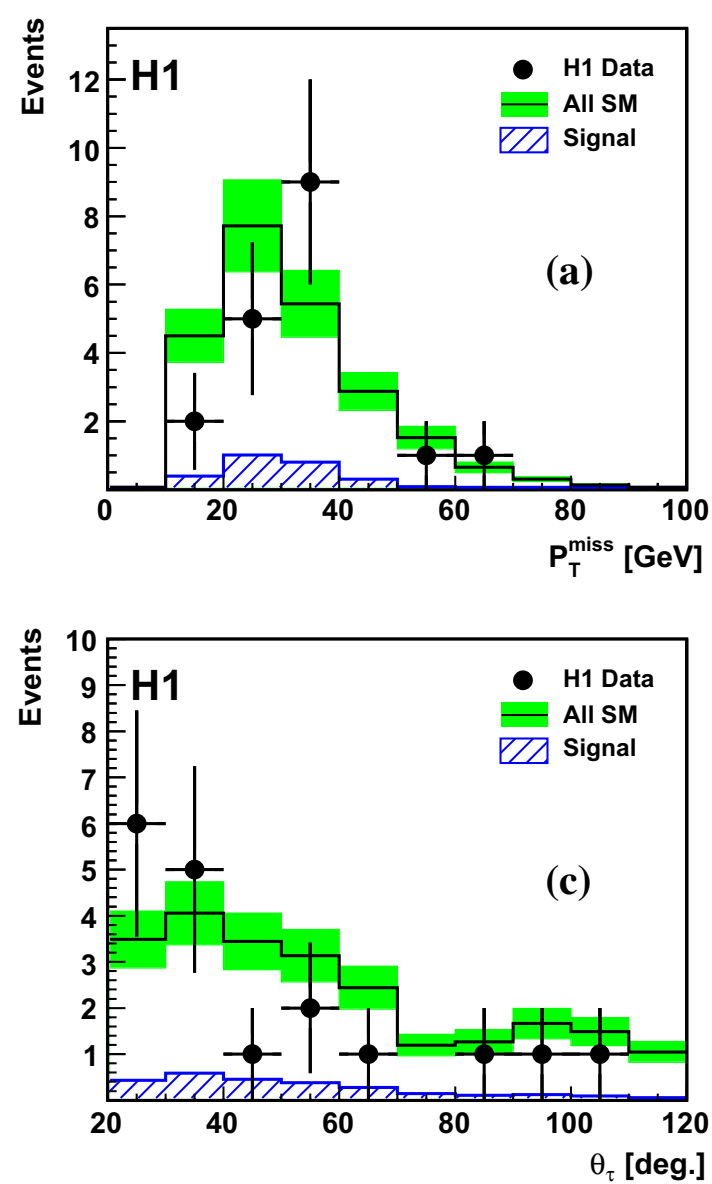

Fig. 41 Distributions in the tau channel for the $\mathrm{H} 1 e^{ \pm} p$ data sample. Shown is the missing transverse momentum $P_{T}^{\text {miss }}(\mathbf{a})$, the hadronic transverse momentum not including the tau-jet candidate $P_{T}^{X^{\prime}}(\mathbf{b})$, the polar angle of the tau-jet candidate $\theta_{\tau}$ (c) and the tau-jet candidate

In the ZEUS analysis of their HERA I data, events are selected with higher missing $P_{T}$ with respect to $\mathrm{H} 1, P_{T}^{\text {miss }}>$ $20 \mathrm{GeV}$. Tau candidates are reconstructed with $E_{T}^{\text {jet }}>5 \mathrm{GeV}$
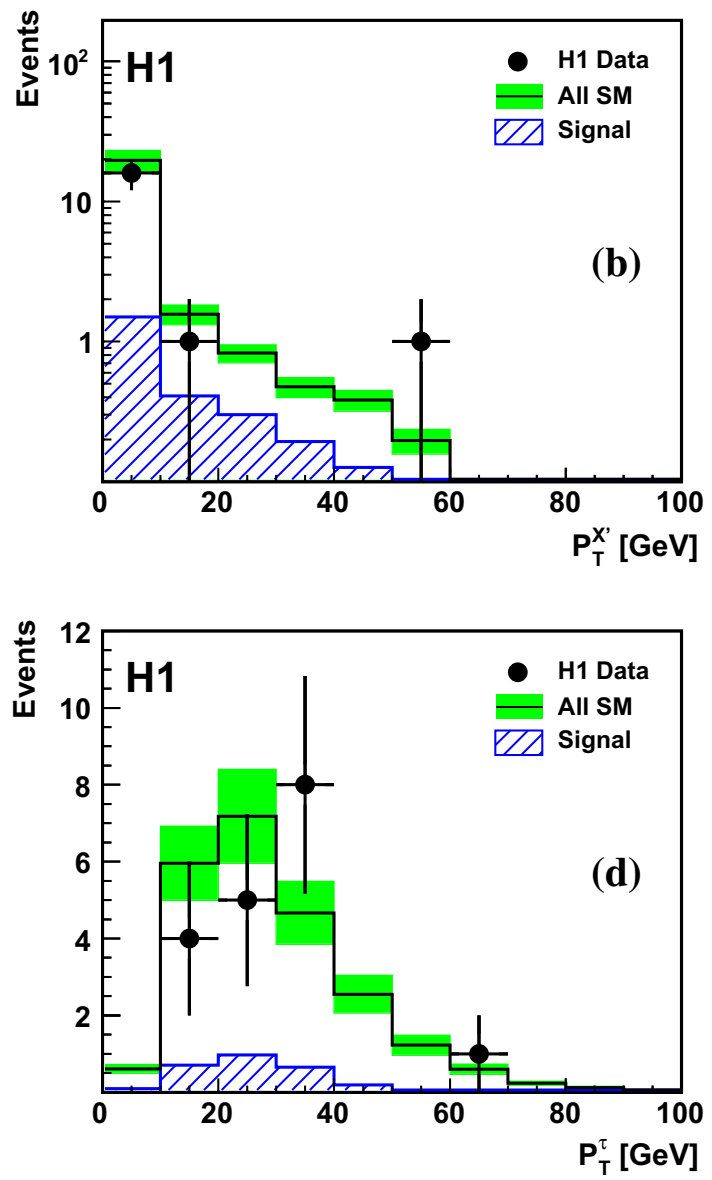

transverse momentum $P_{T}^{\tau}(\mathbf{d})$. The data (points) are compared to the SM expectation (open histogram). The signal component of the SM expectation $\left(W \rightarrow \tau \nu_{\tau}\right)$ is shown as the hatched histogram. The total uncertainty on the SM expectation is shown as the shaded band

in the region $-1.0<\eta<2.5$ and are distinguished from the QCD background using a discriminant based on jet-shape observables [205]. 


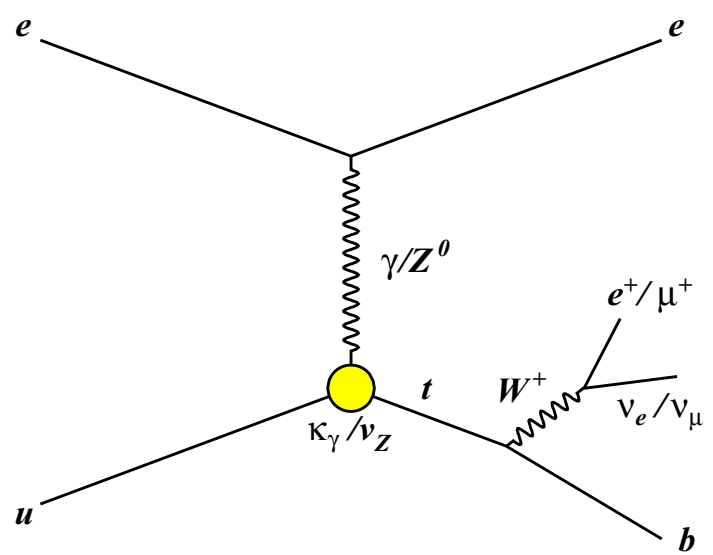

Fig. 42 Anomalous single-top production via flavour-changing neutral current transition with coupling $\kappa_{t} U \gamma$

After applying the discriminant cut, 3 events are observed in the data, compared to SM prediction of $0.40_{-0.13}^{+0.12}$ (where $43 \%$ is due to single- $W$ production). In the region $P_{T}^{X}>$ $25 \mathrm{GeV}, 2$ events are observed compared to an expectation of $0.20 \pm 0.05$. It can therefore be concluded that the data show a general good agreement with the SM predictions and no hint of BSM phenomena is observed at HERA. Recently, a review of single-vector boson production at LHC at $\sqrt{s}=7 \mathrm{TeV}$ has been published [206]. There, all results have been found in agreement between the two main experiments, ATLAS and CMS, and are furthermore consistent with the presently available SM predictions.

\section{Search for single-top production}

The production of single top quarks at HERA is kinematically possible due to the centre of mass energy, which is above the top mass threshold. Within the SM, the dominant process for single-top production is the charged current reaction $e p \rightarrow v t X$ [207-209], which has a tiny cross section of less than $1 \mathrm{fb}[210,211]$, ruling out the observation of SM single-top production at HERA. However, flavour changing neutral current (FCNC) processes $(u \rightarrow t$ or $c \rightarrow t$, mediated by a neutral vector boson, $\gamma$ or $Z$ ) could lead to a visible single-top production cross section. In several extensions of the SM the top quark is predicted to undergo FCNC interactions [212-215] and the observation of top quarks at HERA would thus be a clear indication of physics beyond the SM.

The diagram for anomalous single-top production via FCNC is shown in Fig. 42, where the top quark coupling to a $U$-type quark via a photon $\left(Z^{0}\right.$ boson) is indicated as $\kappa_{\gamma}\left(v_{Z}\right)$. As the top quark mass is comparable to the centre of mass energy at HERA, the initial-state quark originating from the proton needs to be at a significantly high value of $x$ for single-top production. As the charm-quark density at high $x$ is low compared to the density of the $u$ and $d$ valence quarks, the contribution to the cross section given by charm quarks is neglected. For the same reason, production of antitop quarks is neglected, as this would involve anti-quarks in the initial state.

The anomalous single-top production cross section can be parametrised in terms describing the effect of the two FCNC couplings, $A_{\sigma}$ and $B_{\sigma}$, and of their interference, $C_{\sigma}$ :

$\sigma_{e p \rightarrow e t X}=A_{\sigma} \kappa_{\gamma}^{2}+B_{\sigma} v_{Z}^{2}+C_{\sigma} \kappa_{\gamma} v_{Z}$.

Simulation of the anomalous single-top signal is done using the package CompHEP [216], which is also used to determine the parameters $A_{\sigma}, B_{\sigma}$ and $C_{\sigma}$. The interference parameter $C_{\sigma}$ has only a small effect, producing variations of the cross section of less than $1 \%$ in the full considered range of couplings and is therefore neglected.

CompHEP is also used to determine the top-decay widths in the different channels:

$$
\begin{aligned}
\Gamma_{t \rightarrow u \gamma} & =A_{\Gamma} \kappa_{\gamma}^{2}, \\
\Gamma_{t \rightarrow u Z} & =B_{\Gamma} v_{Z}^{2}, \\
\Gamma_{t \rightarrow q W} & =C_{\Gamma},
\end{aligned}
$$

where $A_{\Gamma}$ and $B_{\Gamma}$ are the partial widths of the top corresponding to $u \gamma$ and $u Z$ unitary FCNC couplings and $C_{\Gamma}$ is the SM top width.

As any top quark immediately and exclusively decays into a $b$ quark and a $W$ boson, the experimental signature of single-top production at HERA is either the leptonic, $\ell v$, or hadronic, $q \bar{q}, W$-decay products in combination with a high $P_{T}$ hadronic final state from the $b$-jet. In the case of leptonic $W$ decay, this topology is of particular interest as it is the same as the SM $W$ production events discussed in Sect. 12.1, especially those seen with a prominent hadronic final state. In the case of hadronic $W$ decay, the experimental signature of single-top production is the presence of three jets in an event, with a mass compatible with that of the top quark. Both $\mathrm{H} 1$ and ZEUS have previously investigated FCNC using their HERA I data sets $[194,217]$ and have published searches for single-top events examining the HERA II data sample [218,219].

The main SM background to single-top production in the $W$ leptonic decay channel is from real $W$ production, which has a cross section of about $1 \mathrm{pb}$ and is modelled using the EPVEC MC generator, which is reweighted to NLO QCD. In the $W$ hadronic decay channel, the main background contribution arises from multi-jet production in photoproduction and NC and CC DIS, which are modelled using PYTHIA, RAPGAP and DJANGOH.

Single-top production is investigated by H1, employing both leptonic and hadronic $W$ decays [218]. In the leptonic channel, isolated electrons and muons with a transverse momentum $P_{T}^{\ell}>10 \mathrm{GeV}$ in the polar angle range 
Search for Single Top Quarks at HERA $\left(474 \mathrm{pb}^{-1}\right)$

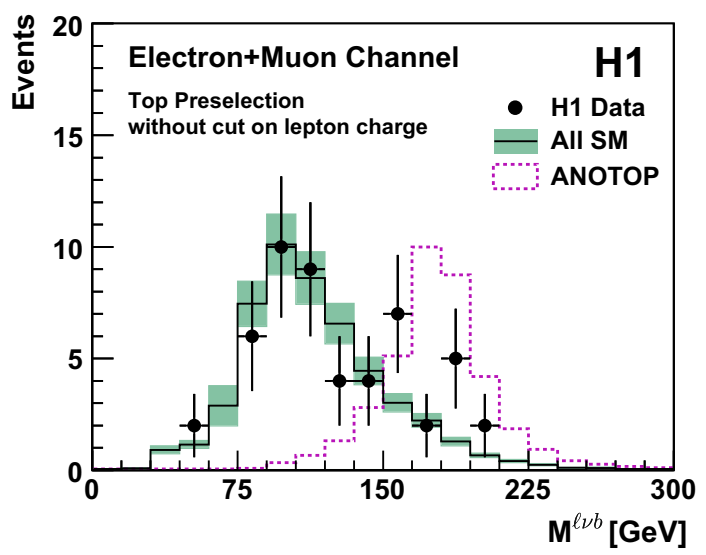

Fig. 43 The distribution of the reconstructed top mass $M^{l v b}$ in the H1 electron and muon channels, after neutrino reconstruction but before the cut on the lepton charge. The data are shown as points, the total SM expectation as the open histogram with systematic and statistical uncertainties added in quadrature (shaded band). The prediction from ANOTOP is also shown with arbitrary normalisation (dashed histogram)

$5^{\circ}<\theta_{l}<140^{\circ}$ are selected in events with a missing transverse momentum $P_{T}^{\text {miss }}>12 \mathrm{GeV}$, where the selection is based on the $\mathrm{H} 1$ analysis described in Sect. 12.1, where 39 (14) electron (muon) events are observed in the data, compared to a SM prediction of $43.1 \pm 6.0(11.0 \pm 1.8)$ [195].

To estimate a potential top contribution to this sample, a top quark candidate is reconstructed from its decay products (lepton $l$, neutrino $v$ and $b$ quark), and the compatibility with single-top quark production via FCNC was tested using a multivariate discriminant method [218]. The kinematic variables of the neutrino are reconstructed using the transverse and longitudinal momentum balance of the event. The fourmomentum of the $b$-jet is taken as the four-momentum of the hadronic final state and the mass of the top quark is reconstructed as the sum of the four-vectors of the isolated lepton, the neutrino and the hadronic final state. The reconstructed top mass $M^{\ell v b}$ for the electron and muon channel combined is shown in Fig. 43, where the data are in overall agreement with the SM prediction, and a slight excess of data events is observed in the top quark mass range. The prediciton from ANOTOP [217], an anomolous top MC MC generator, is also shown with arbitrary normalisation.

The results of the multivariate discriminant neural-net based analysis are cross checked against a cut-based top selection, requiring the selected top events to have a $b$-jet with transverse momentum $P_{T}^{b}>30 \mathrm{GeV}$ and $M^{\ell v b}>140 \mathrm{GeV}$. In the final selection, five (four) events are observed in the electron (muon) channel, compared to a SM expectation of $3.2 \pm 0.4(2.1 \pm 0.3)$.

To investigate the $W$ hadronic decay channel, events are selected by $\mathrm{H} 1$ containing at least three jets in the pseudo- rapidity range $-0.5<\eta_{\text {jet }}<2.5$, with $P_{T}^{\text {jet1 }}>40 \mathrm{GeV}$, $P_{T}^{\text {jet2 }}>30 \mathrm{GeV}$ and $P_{T}^{\text {jet3 }}>15 \mathrm{GeV}$, where the jets are ordered in magnitude of their transverse momenta. Two of the jets are required to have a mass compatible with that of the $W$ boson, within the experimental resolution: $65<$ $M^{\mathrm{jj}}<95 \mathrm{GeV}$. The remaining jet is assumed to be that coming from the $b$ quark and is required to have $P_{T}>25 \mathrm{GeV}$. After the preselection, 404 events are selected, compared to a SM prediction of $388 \pm 32$. Like in the analysis of the leptonic decay channels, a multivariate discriminant is used to differentiate the signal from the background, and the analysis is cross checked with a cut-based selection.

The $b$-jet candidate is required have large transverse momentum $P_{T}^{b}>40 \mathrm{GeV}$ and the reconstructed top quark mass must lie in the range $150<M^{\text {jets }}<210 \mathrm{GeV}$. The number of candidate events selected is 128 , compared to $123 \pm 13$ expected from SM processes.

A similar analysis is performed by ZEUS on the leptonic decay channels of the $W$ boson using their HERA II data sample [219]. Events are selected with $P_{T}^{\text {miss }}>10 \mathrm{GeV}$ (12 GeV for electron events), containing isolated electrons with $P_{T}^{e}>10 \mathrm{GeV}$ in the angular region $17^{\circ}<\theta_{e}<115^{\circ}$, or isolated muons with $P_{T}^{\mu}>8 \mathrm{GeV}$ in the angular region $11^{\circ}<\theta_{\mu}<157^{\circ}$.

In the electron (muon) channel, a total of 245 (269) events were observed, compared to $253 \pm 6(260 \pm 3)$ expected from the SM. A cut on the transverse momentum of the hadronic final state, $P_{T}^{X}>40 \mathrm{GeV}$, is then applied to the data, resulting in a final sample containing 1 event in the electron channel compared to a SM prediction of $3.6 \pm 0.6$, and 3 events in the muon channel, compared to a SM prediction of $3.0 \pm 0.4$.

The number of selected events in each of the $W$-decay channels examined by $\mathrm{H} 1$ and ZEUS is summarised in Table 11, compared to the SM expectation. As no significant deviation from the SM is observed, upper limits on the singletop production cross section are derived using the method of fractional event counting [151]. For all channels combined the $\mathrm{H} 1$ upper limit on the cross section for single-top quark production $95 \% \mathrm{CL}$ is

$\sigma(e p \rightarrow e t X, \sqrt{s}=319 \mathrm{GeV})<0.25 \mathrm{pb}$,

where the limit is reported at $\sqrt{s}=319 \mathrm{GeV}$, taking into account [218] the ratio of 0.70 of the predicted cross sections at $\sqrt{s}=301$ and $319 \mathrm{GeV}$ [220].

The ZEUS analysis described above is based on the HERA II data sample only, and the results are then combined with those of the ZEUS publication on their HERA I data [194] which also includes the hadronic decay channel of the $W$ boson. The resulting ZEUS limit at $95 \% \mathrm{CL}$ on the single-top production cross section is

$\sigma(e p \rightarrow e t X, \sqrt{s}=315 \mathrm{GeV})<0.13 \mathrm{pb}$, 
Table 11 Summary of the H1 and ZEUS searches for single-top production in FCNC at HERA, where the observed number of events in each of the different $W$-decay channels is shown. The number of events predicted by the SM is also shown, where the quoted errors contain statistical and systematic uncertainties added in quadrature

\begin{tabular}{lcc}
\hline \multicolumn{3}{l}{ Search for single-top production in FCNC at HERA } \\
\hline Decay channel & Data & Total SM \\
\hline $\mathrm{H} 1$ analysis $\left(\mathcal{L}=474 \mathrm{pb}^{-1}\right)$ & 5 & $3.2 \pm 0.4$ \\
$W \rightarrow e v_{e}$ & 4 & $2.1 \pm 0.3$ \\
$W \rightarrow \mu v_{\mu}$ & 128 & $123 \pm 13$ \\
$W \rightarrow q q$ & & \\
ZEUS analysis $\left(\mathcal{L}=370 \mathrm{pb}^{-1}\right)$ & 1 & $3.6 \pm 0.6$ \\
$W \rightarrow e v_{e}$ & 3 & $3.0 \pm 0.4$ \\
$W \rightarrow \mu v_{\mu}$ & &
\end{tabular}

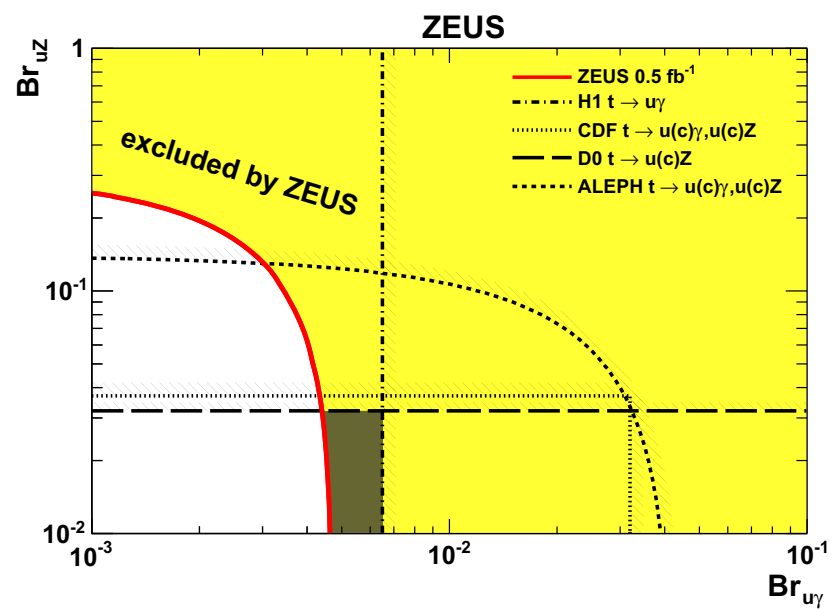

Fig. 44 Exclusion limits set by the $\mathrm{H} 1$ and ZEUS experiments on the branching ratios $\operatorname{Br}(t \rightarrow u \gamma)$ and $\operatorname{Br}(t \rightarrow u Z)$ from the search for single-top production at HERA. Limits from other experiments are also shown

reported at the average centre of mass energy $\sqrt{s}=315 \mathrm{GeV}$ of the complete $0.5 \mathrm{fb}^{-1}$ ZEUS data sample.

The limits on the cross sections are converted into $95 \% \mathrm{CL}$ limits on the anomalous FCNC coupling $k_{\gamma}$ using Eq. (46), which are found by H1 (ZEUS) to be $\kappa_{\gamma}<0.18\left(\kappa_{\gamma}<\right.$ 0.13 ) for a top mass of $175 \mathrm{GeV}$. These limits may in turn be transformed [219] into limits on the branching ratios $\mathrm{Br}(t \rightarrow u \gamma)$ and $\mathrm{Br}(t \rightarrow u Z)$ and $\mathrm{H} 1$ (ZEUS) sets a limit of $\operatorname{Br}(t \rightarrow u \gamma)<0.64 \%(<0.45 \%)$ for low values of $\operatorname{Br}(t \rightarrow u Z)$. The limits on these branching ratios are displayed in Fig. 44, compared to those from other experiments ALEPH [221] at LEP and CDF [222,223] and DØ [224] at the Tevatron.

Once again, the extended reach of the LHC data has extended these limits into a new domain, where searches by the ATLAS [225,226] and CMS [227] Collaborations now limit these branching fractions to sub-permille levels.

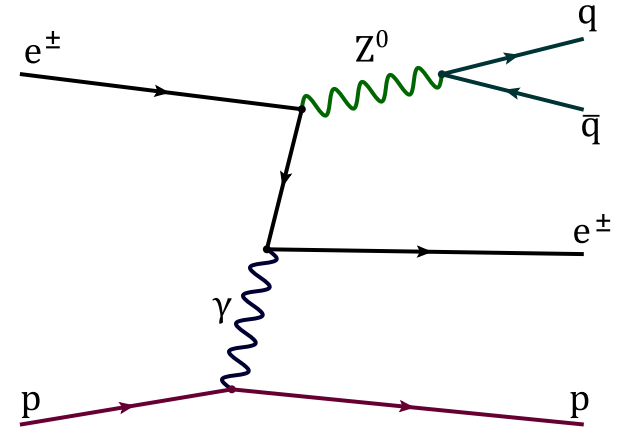

Fig. 45 Example of a leading-order diagram of $Z^{0}$ boson production, $e p \rightarrow e Z^{0} p$ and subsequent hadronic decay into a quark $q$ and an anti-quark $\bar{q}$

\section{$14 Z^{0}$ production at HERA}

Although the number of $W$ or $Z^{0}$ bosons produced at HERA is expected to be small, their study at HERA provides the means to test the SM, as some anomalous couplings of these bosons predict an increase in the production cross section. A measurement of the $W$ production cross section at HERA was performed using events with an isolated electron or muon and missing transverse momentum, as discussed in Sect. 12.1, where good agreement was observed with the SM prediction. An analysis of the production of $Z^{0}$ bosons performed by ZEUS is described in the following.

The full data sample collected with the ZEUS detector is used to study the production of $Z^{0}$ bosons in the process $e p \rightarrow e Z^{0} X$. Compared to the analyses described in Sect. 11, which include $Z^{0}$ decays to lepton pairs, this analysis examines the hadronic decay mode of the $Z^{0}$, chosen because of its large branching ratio and to exploit the excellent resolution of the ZEUS hadronic calorimeter. The analysis is restricted to elastic and quasi-elastic $Z^{0}$ production in order to suppress QCD multi-jet background. A diagram for $Z^{0}$ production at LO and the subsequent $Z^{0}$ hadronic decay is shown in Fig. 45. The decay products of the $Z^{0}$ boson form at least two hadronic jets with high transverse energies. No energy deposit is found around the forward direction, in contrast to what would be expected in inelastic collisions.

The production of $Z^{0}$ bosons is simulated using the EPVEC program to generate the events at the parton level, and PYTHIA to simulate initial- and final-state parton showers. As a reliable simulation for the SM background events, predominantly due to the diffractive photoproduction of jets of high transverse momentum, was not available, the background distributions are estimated from the data as described below.

The event selection is performed requiring the presence of at least two jets in the final state with $E_{T}>25 \mathrm{GeV}$ and $|\eta|<2$. The two highest $E_{T}$ jets are required to be 
separated by at least 2 radians in the azimuthal plane, as the two leading jets from the $Z^{0}$ boson decays are expected to be nearly back-to-back in the $x-y$ plane.

To identify high $Q^{2}$ events, the electron produced in the DIS scattering in the $e p \rightarrow e Z^{0} X$ process is required to be reconstructed in the final state. The electron is required to have an energy $E_{e}^{\prime}>5 \mathrm{GeV}$ and a track with momentum $p_{\text {track }}>3 \mathrm{GeV}$, if found in the acceptance region of the tracking system. Additional cuts are applied in order to suppress the background from low $Q^{2}$ and photoproduction events, as well as background from beam-gas and cosmic interactions. To select elastic and quasi-elastic processes, a cut on $\eta_{\max }<3.0$ is introduced, where $\eta_{\max }$ is defined as the pseudorapidity of the energy deposit in the calorimeter closest to the proton-beam direction with energy greater than $400 \mathrm{MeV}$ as determined by calorimeter cells. This cut also rejects signal events with energy deposits from the scattered electron in the calorimeter around the forward beam pipe, causing an acceptance loss of about $30 \%$.

After all selection cuts are applied, 54 events remain in the final data sample. The total selection efficiency, estimated using the MC simulation, is found to be $22 \%$ for elastic and quasi-elastic processes and less than $1 \%$ for DIS and photoproduction events. The number of signal and background events is estimated using the $M_{\text {jets }}$ distribution as measured in the data. To increase the statistics of the sample, the shape of the $M_{\text {jets }}$ distribution outside the $Z^{0}$ mass region is estimated from the inelastic data, obtained by removing the $\eta_{\max }$ cut, after verification that this cut does not distort the distribution. In this way, the $M_{\text {jets }}$ distribution in the inelastic region is adopted as a background template in a fit to the data in the elastic region, allowing the determination of the number of the signal events and therefore the cross section. The $M_{\text {jets }}$ distribution of the selected events and the fit results are shown in Fig. 46.

The number of observed $Z^{0}$ events is measured to be $15.0_{-6.4}^{+7.0}$ (stat.), corresponding to a statistical significance of the signal of $2.3 \sigma$. The cross section for the elastic and quasi-elastic production of $Z^{0}$ bosons, ep $\rightarrow e Z^{0} X$, at $\sqrt{s}=318 \mathrm{GeV}$, is measured as

$\sigma_{Z^{0}}=0.13 \pm 0.06$ (stat.) \pm 0.01 (sys.) pb,

in agreement with the SM prediction of $0.16 \mathrm{pb}$.

\section{A general search for new phenomena}

The searches for new physics reported so far in this review focus on the highest energy regions accessible at HERA, as a large variety of possible extensions to the SM predict new phenomena, which may appear there. Modelindependent searches are performed without looking for

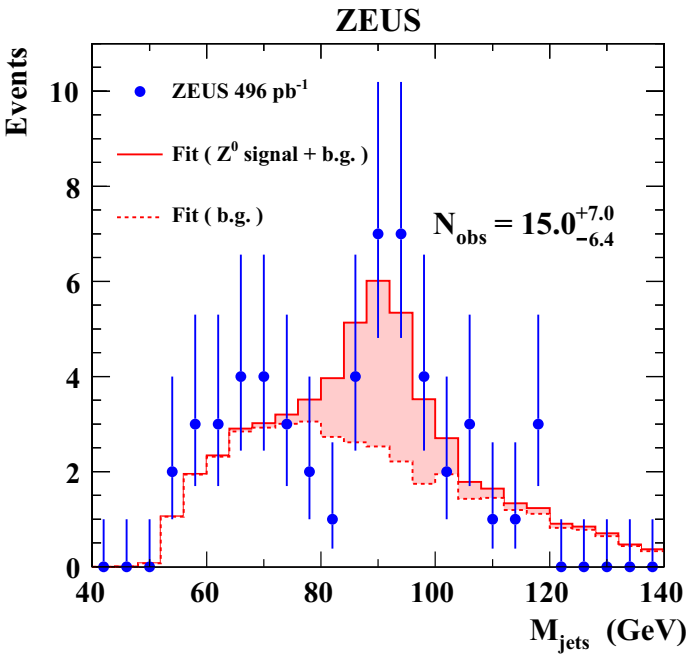

Fig. 46 The $M_{\text {jets }}$ distribution and the fit result. The data are shown as points, and the fitting result of signal+background (background component) is shown as solid (dashed) line. The signal contribution is also indicated by the shaded area and amounts to a total number of $N_{\text {obs }}$ events. The error bars represent the approximate $68 \% \mathrm{CL}$ intervals, calculated as $\pm \sqrt{n+0.25}+0.5$ for a given entry $n$

signatures as predicted by a particular model of BSM physics, but just looking for deviations between the predicted and measured cross sections in regions in which the SM predictions are reliable. Such model-independent analyses do not rely on any a priori definition of expected signatures for exotic phenomena. Therefore, they address the important question of whether unexpected phenomena may occur through a new pattern, not predicted by the SM.

In this section, a more general, model-independent approach is described. The analysis is performed by the $\mathrm{H} 1$ Collaboration using their complete $e^{ \pm} p$ data set [228], corresponding to an integrated luminosity of $463 \mathrm{pb}^{-1}$, of which $178 \mathrm{pb}^{-1}$ was recorded in $e^{-} p$ collisions and $285 \mathrm{pb}^{-1}$ in $e^{+} p$ collisions. Following the analysis strategy of a previous $\mathrm{H} 1$ publication which used only the HERA I data set [229], a search for differences between the observed number of data events and the SM expectation in a large variety of different event topologies is performed. The analysis includes all high $P_{T}$ final-state topologies involving electrons $(e)$, muons $(\mu)$, jets $(j)$, photons $(\gamma)$ or neutrinos $(v)$ and searches for deviations from the SM prediction in phase-space regions where the SM predictions are reliable. The identification of such particles follows that described in Sect. 5.

Calorimetric energy deposits and tracks are used to identify electron, photon and muon candidates. Electron and photon candidates are characterised by compact and isolated electromagnetic showers in the LAr calorimeter with an associated track from the inner tracking systems in the 
case of electrons or with no associated track in the case of photons. The identification of muon candidates is based on a track measured in the inner tracking systems associated with signals in the muon detectors. Calorimetric energy deposits and tracks not previously associated to identified electron and muons are used to reconstruct jets using the inclusive $k_{\mathrm{T}}$ algorithm. If the event contains large $P_{\mathrm{T}}^{\text {miss }}$ it is associated to an outgoing neutrino candidate and the neutrino four-momentum is calculated assuming transverse momentum conservation and using the relation in Eq. (13). A detailed description of the further identification criteria applied to each type of particle can be found in the H1 publication [228]. Such criteria typically include strict isolation requirements and employ information from multiple detector components to ensure a high efficiency and to reduce misidentification.

A precise estimate of all processes relevant at high transverse momentum in $e p$ interactions is needed to ensure a reliable comparison to the SM. Several MC generators, already introduced for the analyses described in the previous sections, are therefore combined to simulate events in all classes. The dominant SM processes at high transverse momenta are NC DIS, which is simulated using RAPGAP and two-jet photoproduction, simulated using PYTHIA. Charged current DIS events are simulated using DJANGOH and contributions from elastic and quasi-elastic QED Compton scattering are simulated with the WABGEN generator. Smaller contributions arising from the production of single $W$ bosons and multi-lepton events are modelled using the EPVEC and GRAPE event generators, respectively.

The common phase space for all the selected particles is defined as $10^{\circ}<\theta<140^{\circ}$ and $P_{T}>20 \mathrm{GeV}$, except for the neutrino, for which the phase space is defined as $P_{T}^{\text {miss }}>20 \mathrm{GeV}$ and $\delta<48 \mathrm{GeV}$. All identified particles with $P_{T}>20 \mathrm{GeV}$, including the neutrino (where the $P_{T}$ is taken from its reconstructed four-vector), are required to be isolated with respect to each other by a minimum distance in pseudorapidity-azimuth of $R>1$. All particles satisfying these requirements are referred to as bodies, and events are classified into exclusive classes according to the number and the types of bodies they contain, for example $e-j, \mu-v-j$, $j-j-j-j$ and so on. All possible classes with at least two bodies are investigated, with the exception of the $\mu \nu$ class, which originates mainly from poorly reconstructed muons giving rise to missing transverse momentum.

The event yields for all the event classes with observed events or with SM expectations greater than 0.01 are given in Table 12. The class with the lowest SM expectation is the $\mu-\mu-v$, where the number of expected events given by the SM MC is $0.010 \pm 0.005$. The yields are given for the full $e^{ \pm} p$ data sample and good agreement is observed between the data and the SM prediction. The same yields are shown in Fig. 47, separately for $e^{+} p$ and $e^{-} p$ collisions. As expected, events with an electron (neutrino) and two or more jets are dominated by NC (CC) DIS, while events with two or more jets and no reconstructed leptons are dominated by photoproduction. Event classes where an electron is observed together with a photon, with or without an accompanying jet, arise from QED Compton processes.

Classes where more than one lepton is observed are mainly due to lepton pair production from $\gamma \gamma$ processes, as already seen in the dedicated analyses described in Sect. 11. Compared to the H1 multi-lepton analysis [170], the leptons in the general search are identified in a wider polar angle region, down to $10^{\circ}$ in the forward direction, and required to have higher transverse momenta. All the multi-lepton events reconstructed in the dedicated analysis and falling into the kinematic region of this analysis are also selected in the general search. Similarly, events in which an electron or a muon are reconstructed together with a neutrino, with or without a jet come mainly from single- $W$ boson production, as already seen in the searches for events with high $P_{T}$ leptons and missing transverse momentum described in Sect. 12.

In addition to the yields, the selected events are also analysed in terms of their topology. The distributions of the transverse momenta of all bodies, $\sum P_{T}$, and the invariant mass of all reconstructed bodies, $M_{\text {all }}$, are analysed together with their angular distributions and energy ratios, which are sensitive to spin and decay properties of hypothetical high-mass particles. The variables employed are defined inspired by topological analyses of multi-jet events [230] and use the so-called main body [228] of the event. Simplifying the picture, this main body is defined according to a priority list between bodies of different types $(\gamma, e, \mu, v, j)$ and using criteria based on its transverse momentum, in the case where two bodies of the same kind are present in the event. The variable $\cos \theta_{\text {lead }}^{*}$ is then defined as the cosine of the polar angle of the leading body relative to the incident proton in the centre of mass frame defined by all bodies. The variable $X_{\text {lead }}$ is the energy fraction of the leading body and is defined for systems with three or more bodies as

$X_{\text {lead }}=\frac{2 E_{\text {lead }}^{*}}{\sum_{i} E_{i}^{*}}$

where the sum runs over all bodies and the energies are evaluated in the centre of mass frame of all bodies. The sensitivity of $\cos \theta_{\text {lead }}^{*}$ and $X_{\text {lead }}$ to new physics was tested using MC samples of various exotics processes, such as leptoquarks, excited fermions or anomalous top production. The distribution of the variables $\sum P_{T}, M_{\text {all }}, \cos \theta_{\text {lead }}^{*}$ and $X_{\text {lead }}$ from the data are compared to SM predictions and, as also seen in the event yields in Table 12, good agreement is found in all cases [228]. An example is given in Fig. 48, where a clear difference is observed between the $\cos \theta_{\text {lead }}^{*}\left(X_{\text {lead }}\right)$ distribution 
Table 12 Observed and predicted event yields for all event classes with observed data events or a SM expectation greater than 0.01 for all $e^{ \pm} p$ data. Each event class is labelled with the leading body listed first. The uncertainties on the predictions include model uncertainties and exper- imental systematic errors added in quadrature. The $\hat{P}$ values (see text) obtained in the scan of $\sum P_{T}, M_{\text {all }}, \cos \theta_{\text {lead }}^{*}$ and $X_{\text {lead }}$ distributions are also given

H1 general search for new phenomena $\left(e^{ \pm} p\right.$ collisions, $\left.\mathcal{L}=463 \mathrm{pb}^{-1}\right)$

\begin{tabular}{|c|c|c|c|c|c|c|}
\hline Event class & Data & Total SM & $\hat{P}_{\sum P_{T}}$ & $\hat{P}_{M_{\text {all }}}$ & $\hat{P}_{\cos \theta_{\text {lead }}^{*}}$ & $\hat{P}_{X_{\text {lead }}}$ \\
\hline$j-j$ & 156724 & $153278 \pm 27400$ & 0.57 & 0.33 & 0.98 & \\
\hline$e-j$ & 125900 & $127917 \pm 15490$ & 0.090 & 0.99 & 0.40 & \\
\hline$\mu-j$ & 21 & $19.5 \pm 3.0$ & 0.30 & 0.46 & 0.024 & \\
\hline$v-j$ & 11081 & $11182 \pm 1165$ & 0.33 & 0.31 & 0.25 & \\
\hline$e-v$ & 16 & $21.5 \pm 3.5$ & 0.13 & 0.084 & 0.62 & \\
\hline$e-e$ & 36 & $40.0 \pm 3.7$ & 0.35 & 0.041 & 0.52 & \\
\hline$e-\mu$ & 19 & $21.0 \pm 2.1$ & 0.46 & 0.83 & 0.81 & \\
\hline$\mu-\mu$ & 18 & $17.5 \pm 3.0$ & 0.31 & 0.50 & 0.88 & \\
\hline$\gamma-j$ & 563 & $538 \pm 86$ & 0.31 & 0.21 & 0.77 & \\
\hline$\gamma-e$ & 619 & $648 \pm 62$ & 0.93 & 0.99 & 0.10 & \\
\hline$\gamma-\mu$ & 0 & $0.22 \pm 0.04$ & 1 & 1 & 1 & \\
\hline$\gamma-v$ & 4 & $9.6 \pm 2.8$ & 0.076 & 0.33 & 0.22 & \\
\hline$\gamma-\gamma$ & 1 & $1.1 \pm 0.6$ & 0.66 & 0.35 & 0.11 & \\
\hline$j-j-j$ & 2581 & $2520 \pm 725$ & 0.54 & 0.65 & & 0.18 \\
\hline$e-j-j$ & 1394 & $1387 \pm 270$ & 0.0044 & 0.70 & & 0.28 \\
\hline$\mu-j-j$ & 1 & $0.46 \pm 0.18$ & 0.12 & 0.072 & & 0.99 \\
\hline$v-j-j$ & 355 & $338 \pm 62$ & 0.80 & 0.48 & & 0.62 \\
\hline$e-e-j$ & 0 & $0.31 \pm 0.04$ & 1 & 1 & & 1 \\
\hline$e-e-v$ & 0 & $0.06 \pm 0.01$ & 1 & 1 & & 1 \\
\hline$e-e-e$ & 1 & $0.22 \pm 0.04$ & 0.15 & 0.031 & & 0.14 \\
\hline$\mu-\mu-j$ & 0 & $0.16 \pm 0.03$ & 1 & 1 & & 1 \\
\hline$e-\mu-\mu$ & 0 & $0.37 \pm 0.07$ & 1 & 1 & & 1 \\
\hline$\mu-\mu-v$ & 0 & $0.010 \pm 0.005$ & 1 & 1 & & 1 \\
\hline$e-\mu-j$ & 0 & $0.16 \pm 0.04$ & 1 & 1 & & 1 \\
\hline$e-v-j$ & 4 & $3.2 \pm 0.5$ & 0.24 & 0.57 & & 0.095 \\
\hline$\mu-v-j$ & 5 & $2.8 \pm 0.5$ & 0.27 & 0.30 & & 0.35 \\
\hline$e-\mu-v$ & 0 & $0.05 \pm 0.01$ & 1 & 1 & & 1 \\
\hline$\gamma-j-j$ & 5 & $6.7 \pm 1.3$ & 0.41 & 0.25 & & 0.91 \\
\hline$\gamma-e-j$ & 12 & $19.4 \pm 4.0$ & 0.31 & 0.28 & & 0.53 \\
\hline$\gamma-v-j$ & 1 & $4.5 \pm 1.5$ & 0.35 & 0.62 & & 0.47 \\
\hline$e-j-j-j$ & 19 & $22 \pm 6.5$ & 0.84 & 0.80 & & 0.14 \\
\hline$v-j-j-j$ & 7 & $5.2 \pm 1.4$ & 0.47 & 0.39 & & 0.017 \\
\hline$\gamma-v-j-j$ & 0 & $0.16 \pm 0.07$ & 1 & 1 & & 1 \\
\hline$e-v-j-j$ & 0 & $0.15 \pm 0.09$ & 1 & 1 & & 1 \\
\hline$\gamma-e-j-j$ & 0 & $0.22 \pm 0.07$ & 1 & 1 & & 1 \\
\hline$e-e-v-j$ & 0 & $0.10 \pm 0.06$ & 1 & 1 & & 1 \\
\hline$e-\mu-v-j$ & 0 & $0.08 \pm 0.05$ & 1 & 1 & & 1 \\
\hline$j-j-j-j$ & 40 & $33 \pm 13$ & & & & \\
\hline$e-j-j-j-j$ & 1 & $0.13 \pm 0.06$ & & & & \\
\hline$v-j-j-j-j$ & 1 & $0.05 \pm 0.02$ & & & & \\
\hline$j-j-j-j-j$ & 0 & $0.14 \pm 0.09$ & & & & \\
\hline
\end{tabular}


Fig. 47 The data and the SM expectation for all event classes in the $\mathrm{H} 1$ general search with observed data events or a SM expectation greater than 0.01 events for $e^{+} p$ collisions (left) and $e^{-} p$ collisions (right). The error bands on the predictions include model uncertainties and experimental systematic uncertainties added in quadrature

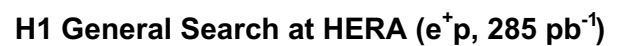

H1 General Search at HERA (e p, $\left.178 \mathrm{pb}^{-1}\right)$
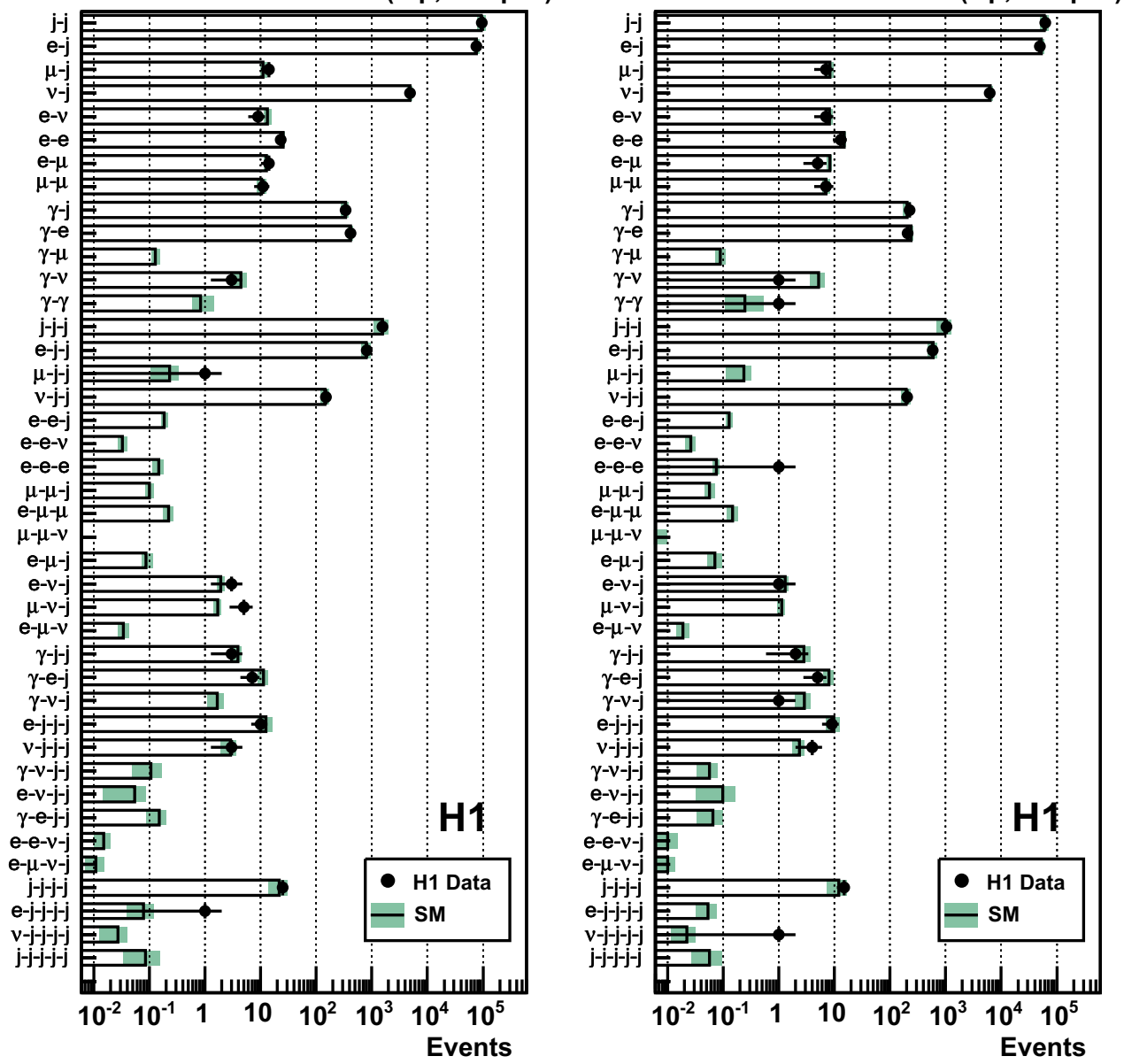

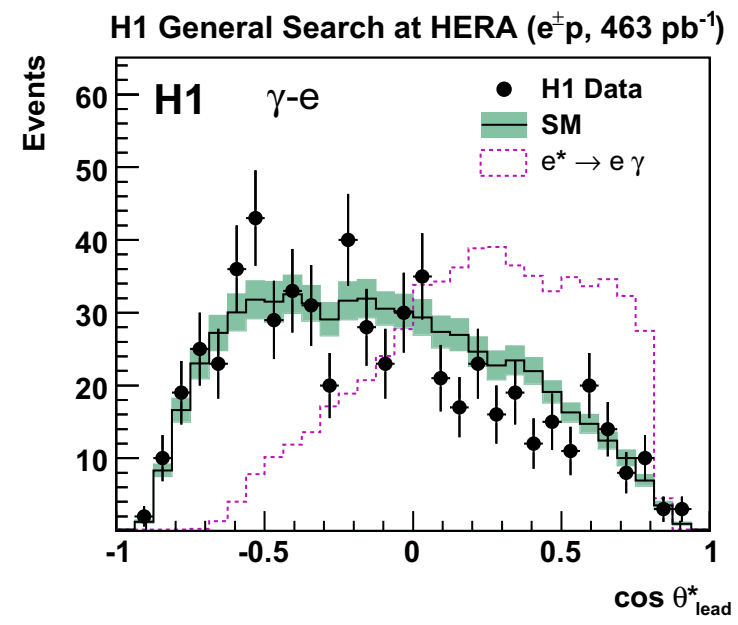

Fig. 48 The $\cos \theta_{\text {lead }}^{*}$ distribution in the $\gamma-e$ event class (left) and the $X_{\text {lead }}$ distribution in the $e-j-j$ event class (right) from the H1 general search. The points correspond to the observed data events and the histograms to the SM expectation. The error bands on the SM prediction

of data and an MC simulated exotic $e^{*}$ resonance [231] $\left(v^{*}\right.$ resonance [232]) with a mass of $200 \mathrm{GeV}$, in the $\gamma e(e-j-j)$ event class.

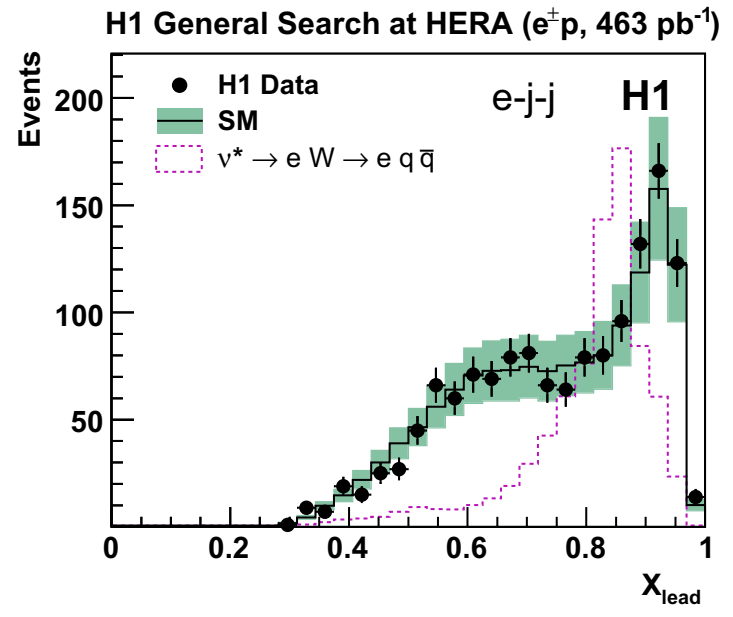

include model uncertainties and experimental systematic uncertainties added in quadrature. The dashed line represents the distribution corresponding to an exotic resonance with a mass of $200 \mathrm{GeV}$, with an arbitrary normalisation

In order to quantify the observed difference between data and SM predictions in terms of agreement or disagreement, a quantity $\hat{P}$ is introduced, according to a search algorithm 
Fig. 49 Diagrams showing the production of excited electrons (a), excited neutrinos (b) and excited quarks (c) in $e p$ collisions at HERA, followed by decays into a SM fermion and a gauge boson

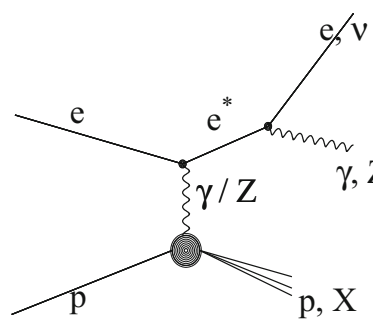

(a)

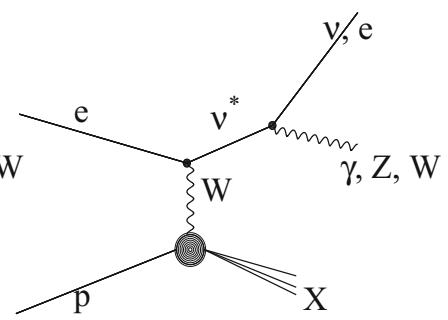

(b)

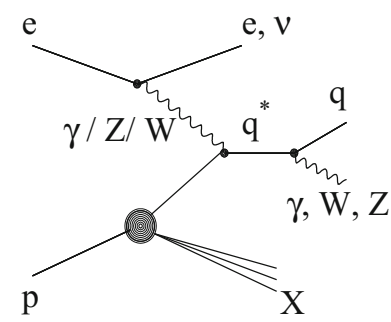

(c) described in the first H1 publication [229]. The quantity $\hat{P}$ is defined such that the smaller the $\hat{P}$ value, the more significant the deviation between data and SM predictions. When $\hat{P}>$ 0.01 , the event class is considered to be in agreement with the SM. The values of $\hat{P}$ for each of the event classes are also shown in Table 12. For some of the classes, such as $j-j-j-j, e-j-j-j-j$ and $v-j-j-j-j$, no reliable $\hat{P}$ values can be calculated due to the uncertainties of the SM predictions, so they are not considered. The fact that small values of $\hat{P}$ can be observed in some classes simply due to the large number of analysed event classes is taken into account in the analysis. The probability of a given value of $\hat{P}$ to be observed in the data is calculated by performing a large number of MC experiments [228].

For the $\sum P_{T}$ and $M_{\text {all }}$ distributions, in general good agreement is observed between the $\hat{P}$ values observed in the data and those obtained from the MC experiments. The most significant deviation from SM is observed in $e^{+} p$ collisions in the $e-e$ class, with $\hat{P}=0.0035$. It is visible in Fig. 47 but not in Table 12, as in the table the event yields are given for the full $e^{ \pm} p$ data sample. This corresponds to five data events being observed in the invariant mass region $110<M_{\text {all }}<120 \mathrm{GeV}$, where the SM expectation is $0.43 \pm 0.04$. This deviation has already been observed in the $\mathrm{H} 1$ analysis described in Sect. 11. The global probability to find at least one class in the $e^{+} p$ data sample with a smaller value of $\hat{P}$ is $12 \%$ as extracted from MC experiments. It is, however, interesting to see that this very general analysis, performed with a completely different method, finds as its most striking deviation from the SM an event class already identified as interesting in a dedicated analysis, focussed on that particular topology. In the case of the $\cos \theta_{\text {lead }}^{*}$ and $X_{\text {lead }}$ distributions, no significant deviation with respect to the SM is observed. The full analysis is also performed at lower $\left(P_{T}>15 \mathrm{GeV}\right)$ and higher $\left(P_{T}>40 \mathrm{GeV}\right)$ transverse momenta, and a good overall agreement between data and the SM is also found in these regions.

This general analysis of high transverse momentum, highmass events, comprising different kind of particles in the final states, demonstrates a very good understanding of the high $P_{T}$ phenomena recorded at the HERA collider. It also confirms a slight deviation already observed in a less general model-independent analysis, the search for multi-lepton events, quantifying the probability of observing such a deviation as $12 \%$.

\section{Searches for excited fermions}

The existence of excited states of leptons and quarks is a natural consequence of models of composite fermions [233], and their discovery would provide convincing evidence of a new scale of matter. The high-energy electron-proton interactions at HERA provide a good environment in which to search for excited states of first generation fermions. Several results on searches for excited fermions have been published by both H1 and ZEUS using partial HERA data sets [234-237]. In this review we focus on the $\mathrm{H} 1$ results $[231,232,238]$ that use the full data HERA sample, corresponding to an integrated luminosity of $475 \mathrm{pb}^{-1}$, which comprises $291 \mathrm{pb}^{-1}$ of $e^{+} p$ collisions and $184 \mathrm{pb}^{-1}$ of $e^{-} p$ collisions. The ZEUS analyses are in agreement with that of $\mathrm{H} 1$ within their extracted larger limits.

Leading-order diagrams for gauge-mediated production and decay of excited fermions at HERA are shown in Fig. 49. The single production of an excited electron, $e^{*}$ (excited neutrino, $\left.v^{*}\right)$, in $e p$ collisions may result from the $t$-channel exchange of a photon or $Z^{0}$ boson ( $W$ boson). The single production of an excited quark, $q^{*}$, proceeds predominantly via gauge boson exchange between the incoming electron and a quark from the proton. ${ }^{14}$ Due to the helicity dependence of the weak interaction and the valence quark composition and density distribution of the proton, the $v^{*}$ production cross section is predicted to be much larger for $e^{-} p$ collisions than for $e^{+} p$ and hence only the $e^{-} p$ data set is considered in that analysis.

The production and decay of such particles is primarily studied ${ }^{15}$ by $\mathrm{H} 1$ in gauge-mediated (GM) models [239-241], where the excited fermions are assumed to have spin $1 / 2$ and isospin $1 / 2$ and the left-handed and right-handed com-

\footnotetext{
14 The exchange of excited quarks in the $u$-channel is also possible and relevant to the analysis for high $q^{*}$ masses and low values of the compositeness scale, $\Lambda$.

15 In addition to GM interactions, $e^{*}$ production and decay via contact interactions is also investigated, where it is found to mediate less than $5 \%$ of the decays and is therefore neglected [231].
} 
ponents form the weak iso-doublets $F_{L}^{*}$ and $F_{R}^{*}$. In such models, only the right-handed component of the excited fermion $F_{R}^{*}$ is allowed to couple to light fermions, to prevent the light leptons from radiatively acquiring a large anomalous magnetic moment [242,243]. The resulting effective Lagrangian [240,241] features a compositeness scale $\Lambda$ with units of energy and three form factors $f, f^{\prime}$ and $f_{s}$ associated to the electroweak and strong gauge groups. For a given excited fermion mass, $M_{f^{*}}$, and assuming a numerical relation between $f, f^{\prime}$ and $f_{s}$, the excited fermion branching ratios are fixed and the production cross section depends only on $f / \Lambda$.

As neither the excited electron or excited neutrino are expected to have strong interactions, these searches are insensitive to $f_{s}$ and the assumption is made that the coupling parameters $f$ and $f^{\prime}$ are of comparable strength. For excited leptons the usual complementary coupling assignments $f=+f^{\prime}$ and $f=-f^{\prime}$ are considered, although in the case of excited electrons $f=-f^{\prime}$ means that the $e^{*}$ does not couple to the photon resulting in a much smaller cross section, and as such only $f=+f^{\prime}$ is considered in that analysis. Only $\gamma, W$ and $Z^{0}$ decays of the $q^{*}$ are considered, so the strong coupling parameter $f_{s}=0$ and the assumption is made that the coupling parameters $f$ and $f^{\prime}$ are of comparable strength, with the relation $f=f^{\prime}$.

Excited fermion events are simulated using the cross section formulae for GM interactions [240,241] in the CompHEP program [244,245] for $v^{*}$ and $q^{*}$ events, and using COMPOS [246] for $e^{*}$ events. The proton parton densities are taken from the CTEQ5L [127] parametrisation and are evaluated at the scale of the four-momentum transfer squared, $\sqrt{Q^{2}}$, in the case of excited leptons and at $\sqrt{\hat{s}}=\sqrt{s x}$ for excited quarks. CompHEP includes the full transition matrix for the production and decay modes, while COMPOS uses the narrow width approximation for the production of $e^{*}$, also taking into account the natural width of the subsequent $e^{*}$ decay [231].

As can be seen from Fig. 49, a large number of final states are possible due to the decay of the $W$ or $Z^{0}$ produced in the excited fermion decay. Each of these final states, which are listed in Table 13, are analysed separately using dedicated event selections as detailed in the $\mathrm{H} 1$ publications [231,232,238]. These selections are based on the identification of high $P_{T}$ objects in the forward and central regions of the detector such as electrons, muons, photons and jets, as well as missing transverse momentum, similarly to what is done in the general search described in Sect. 15. The event selections are optimised to maximise the signal efficiency in each channel. The analysis of the excited electron decay $e^{*} \rightarrow e \gamma$ is separated into inelastic and elastic parts using the total hadronic energy in the event [231]. When multiple objects of the same type are found in the event, the $P_{T}$ threshold of the second and third objects are generally lower.
Note that $e^{*} \rightarrow \nu W \rightarrow \nu e v$ and $e^{*} \rightarrow \nu Z^{0} \rightarrow e \nu v$ decays produce identical final states and they are therefore examined together.

The main source of SM background in each channel depends on the final state. NC DIS is modelled using RAPGAP and CC DIS is modelled using DJANGOH. Compton events are modelled using WABGEN and the GRAPE (EPVEC) program is used to model the lepton pair ( $W$ production) contribution. Cuts to reduce SM background are applied similarly to what is done in the analysis of events with isolated electrons or muons and missing transverse momentum (see Sect. 12.1), using quantities such as the total $E-P_{z}$ of the event (Eq. (13)) and $\xi_{e}=E_{e} \cos ^{2}\left(\theta_{e} / 2\right)$, which, in NC DIS, is equivalent to the transferred four-momentum squared, $Q^{2}$.

The event yields observed in all decay channels are summarised in Table 13 and are in agreement with the corresponding SM expectations. QED Compton is the main SM background in the $e^{*} \rightarrow e \gamma$ search, whereas NC (CC) DIS dominates the SM prediction in the $e^{*} \rightarrow e Z^{0} \rightarrow e q \bar{q}$ $\left(e^{*} \rightarrow \nu W \rightarrow v q \bar{q}\right)$ channel. In the search for excited neutrinos, the SM predictions are dominated by NC DIS in the $\nu^{*} \rightarrow e W \rightarrow e q \bar{q}$ search and by CC DIS for in the $v^{*} \rightarrow \nu \gamma$ and $v^{*} \rightarrow v Z^{0} \rightarrow v q \bar{q}$ searches. The SM contribution in the $q^{*} \rightarrow q \gamma$ and $q^{*} \rightarrow q W / Z^{0} \rightarrow q q \bar{q}$ channels is mainly due to NC DIS, whereas $W$ production is the main contribution in the $q^{*} \rightarrow q W \rightarrow q e v$ and $q^{*} \rightarrow q W \rightarrow q \mu \nu$ channels. The invariant mass distributions of some of the most populous excited fermion-decay channels are displayed in Fig. 50, where the data distributions are seen to be in good agreement with the SM expectation.

Since no evidence of excited fermions is observed, limits are derived at the $95 \% \mathrm{CL}$ on the scale $f / \Lambda$ as a function of the excited fermion mass $M_{f^{*}}$. For each excited fermion type, the decay channels are combined and the limits are obtained from the mass spectra using a modified frequentist approach, which takes statistical and systematic uncertainties into account [178]. For excited electrons a pure gaugemediated interaction is assumed and for the standard scenario where $f=+f^{\prime}$ and $f / \Lambda=1 / M_{e^{*}}$, excited electrons with a mass lower than $272 \mathrm{GeV}$ are excluded at $95 \% \mathrm{CL}$. With the assumption $f / \Lambda=1 / M_{v^{*}}$ excited neutrinos with masses up to $213 \mathrm{GeV}(196 \mathrm{GeV})$ are excluded for $f=-f^{\prime}$ $\left(f=+f^{\prime}\right)$. Finally, assuming $f=+f^{\prime}$, no strong interactions $f_{s}=0$ and $f / \Lambda=1 / M_{q^{*}}$, excited quarks with a mass lower than $259 \mathrm{GeV}$ are excluded at $95 \% \mathrm{CL}$.

The $\mathrm{H} 1$ limits as a function of excited fermion mass are shown in Fig. 51, where they are compared to limits from LEP (excited electrons: [247,248], excited neutrinos: [249], excited quarks: [250]) and the Tevatron (excited electrons: [251]). Analyses from the LHC by ATLAS and CMS using their $\sqrt{s}=8 \mathrm{TeV}$ data have recently pushed these limits into a new mass regime, where, under the assumption 
Table 13 Summary of the observed and predicted event yields for the various excited fermion-decay channels in the $\mathrm{H} 1$ excited fermion analyses. The uncertainty on the SM prediction includes model and experi- mental systematic uncertainties added in quadrature. Typical selection efficiencies for excited fermion masses ranging from 120 to $260 \mathrm{GeV}$ are also indicated

\begin{tabular}{|c|c|c|c|}
\hline \multicolumn{4}{|c|}{ H1 search for excited fermions at HERA } \\
\hline Channel & Data & Total SM & Signal efficiency $(\%)$ \\
\hline \multicolumn{4}{|c|}{ Excited electrons $\left(e^{ \pm} p\right.$ collisions, $\left.\mathcal{L}=475 \mathrm{pb}^{-1}\right)$} \\
\hline$e^{*} \rightarrow e \gamma($ ela. $)$ & 42 & $48 \pm 4$ & $60-70$ \\
\hline$e^{*} \rightarrow e \gamma$ (inel.) & 65 & $65 \pm 8$ & $60-70$ \\
\hline$e^{*} \rightarrow \nu W \rightarrow \nu q \bar{q}$ & 129 & $133 \pm 32$ & $20-55$ \\
\hline$e^{*} \rightarrow \nu W \rightarrow v e v$ & \multirow{2}{*}{4} & \multirow{2}{*}{$4.5 \pm 0.7$} & 60 \\
\hline$e^{*} \rightarrow e Z^{0} \rightarrow e v v$ & & & 35 \\
\hline$e^{*} \rightarrow e Z^{0} \rightarrow e q \bar{q}$ & 286 & $277 \pm 62$ & $20-55$ \\
\hline$e^{*} \rightarrow e Z^{0} \rightarrow e e e$ & 0 & $0.72 \pm 0.06$ & 60 \\
\hline$e^{*} \rightarrow e Z^{0} \rightarrow e \mu \mu$ & 0 & $0.52 \pm 0.05$ & $40-15$ \\
\hline \multicolumn{4}{|c|}{ Excited neutrinos $\left(e^{-} p\right.$ collisions, $\left.\mathcal{L}=184 \mathrm{pb}^{-1}\right)$} \\
\hline$v^{*} \rightarrow v \gamma$ & 7 & $12.3 \pm 3.0$ & $50-55$ \\
\hline$v^{*} \rightarrow e W \rightarrow e q \bar{q}$ & 220 & $223 \pm 47$ & $40-65$ \\
\hline$\nu^{*} \rightarrow e W \rightarrow e \nu \mu$ & 0 & $0.40 \pm 0.05$ & 35 \\
\hline$\nu^{*} \rightarrow e W \rightarrow e v e$ & 0 & $0.7 \pm 0.1$ & 45 \\
\hline$v^{*} \rightarrow v Z^{0} \rightarrow v q \bar{q}$ & 89 & $95 \pm 21$ & $25-55$ \\
\hline$v^{*} \rightarrow v Z^{0} \rightarrow v e e$ & 0 & $0.19 \pm 0.05$ & 45 \\
\hline \multicolumn{4}{|c|}{ Excited quarks $\left(e^{ \pm} p\right.$ collisions, $\left.\mathcal{L}=475 \mathrm{pb}^{-1}\right)$} \\
\hline$q^{*} \rightarrow q \gamma$ & 44 & $46 \pm 8$ & $35-45$ \\
\hline$q^{*} \rightarrow q W / Z^{0} \rightarrow q q \bar{q}$ & 341 & $326 \pm 78$ & $5-55$ \\
\hline$q^{*} \rightarrow q W \rightarrow q e v$ & 6 & $6.0 \pm 0.8$ & $20-30$ \\
\hline$q^{*} \rightarrow q W \rightarrow q \mu \nu$ & 5 & $4.4 \pm 0.7$ & $20-40$ \\
\hline$q^{*} \rightarrow q Z^{0} \rightarrow q e e$ & 0 & $0.44 \pm 0.08$ & $15-30$ \\
\hline$q^{*} \rightarrow q Z^{0} \rightarrow q \mu \mu$ & 0 & $0.87 \pm 0.11$ & $15-30$ \\
\hline
\end{tabular}

$f=f^{\prime}=1$ and $\Lambda=M_{f^{*}}$, excited electrons (quarks) are ruled out by ATLAS at the 95\% CL for masses lower than $2.2 \mathrm{TeV}$ [252] (3.5 TeV [253]). However, the H1 limits on excited neutrinos currently remain the most stringent at the time of writing.

\section{Searches for supersymmetry}

Supersymmetric (SUSY) extensions of the SM [254] introduce new elementary particles which are the superpartners of SM particles but differ in spin by half a unit $[138,139]$. The hypothesis of the existence of supersymmetric particles has had a huge impact on both theory and experiments. A large variety of new states and a rich phenomenology of the Higgs sector was predicted, which came into focus during the running of the LEP and HERA experiments, and is now thoroughly investigated at the LHC. The masses of the new particles, called sparticles, are related to the symmetry breaking mechanism. A new quantum number $R_{p}=(-1)^{3 B+L+2 S}$ is defined, denoted $R$-parity, where $B$ is the baryon number, $L$ the lepton number and $S$ the spin of a particle. For particles $R_{p}=1$ and for their supersymmetric partners $R_{p}=-1$.

Most collider searches focus on SUSY models that conserve $R$-parity, only allowing the production of sparticles in pairs. However, the most general supersymmetric theory that is renormalisable and gauge invariant with respect to the SM gauge group does not impose $R$-parity conservation $[255,256]$, allowing couplings between two SM fermions and a squark $(\tilde{q})$ or a slepton $(\tilde{l})$. This makes the resonant, single production of sparticles via $R$-parity violating $\left(R_{p}\right)$ couplings possible, and the $e p$ collisions at HERA provide an ideal environment to search for such new particles. Searches have been performed at HERA for three different $R_{p}$ SUSY signatures with squarks, bosonic stop decays and light gravitinos, which are described in the remainder of this section.

17.1 Search for squarks in $\boldsymbol{R}$-parity violating supersymmetry

In SUSY models with $R$-parity violation squarks can couple to electrons and quarks via the $R_{p}$ Yukawa couplings $\lambda_{1 j k}^{\prime}$, 

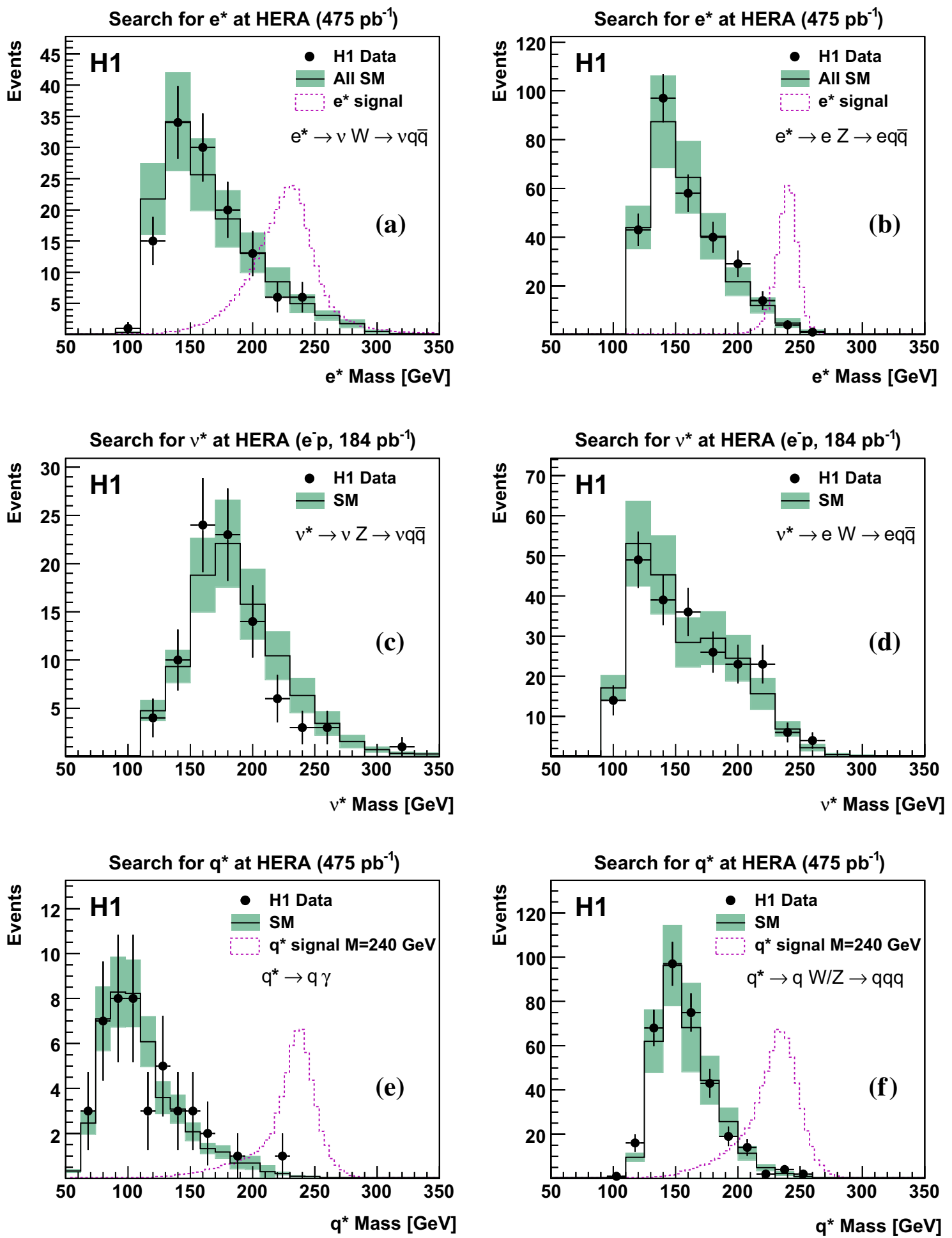

Fig. 50 Invariant mass distribution of the excited fermion candidates in the $e^{*} \rightarrow \nu W \rightarrow v q \bar{q}(\mathbf{a}), e^{*} \rightarrow e Z^{0} \rightarrow e q \bar{q}(\mathbf{b}), v^{*} \rightarrow \nu Z^{0} \rightarrow v q \bar{q}(\mathbf{c})$, $\nu^{*} \rightarrow e W \rightarrow e q \bar{q}(\mathbf{d}), q^{*} \rightarrow q \gamma(\mathbf{e})$ and $q^{*} \rightarrow q W / Z^{0} \rightarrow q q \bar{q}$ (f) search channels. The points correspond to the observed data events and the histograms to the SM expectation after the final selections. The error

bands on the SM prediction include model uncertainties and experimental systematic uncertainties added in quadrature. The dashed line in the $e^{*}$ and $q^{*}$ figures represents the reconstructed mass distribution of excited fermion events with $M_{f^{*}}=240 \mathrm{GeV}$ with an arbitrary normalisation 

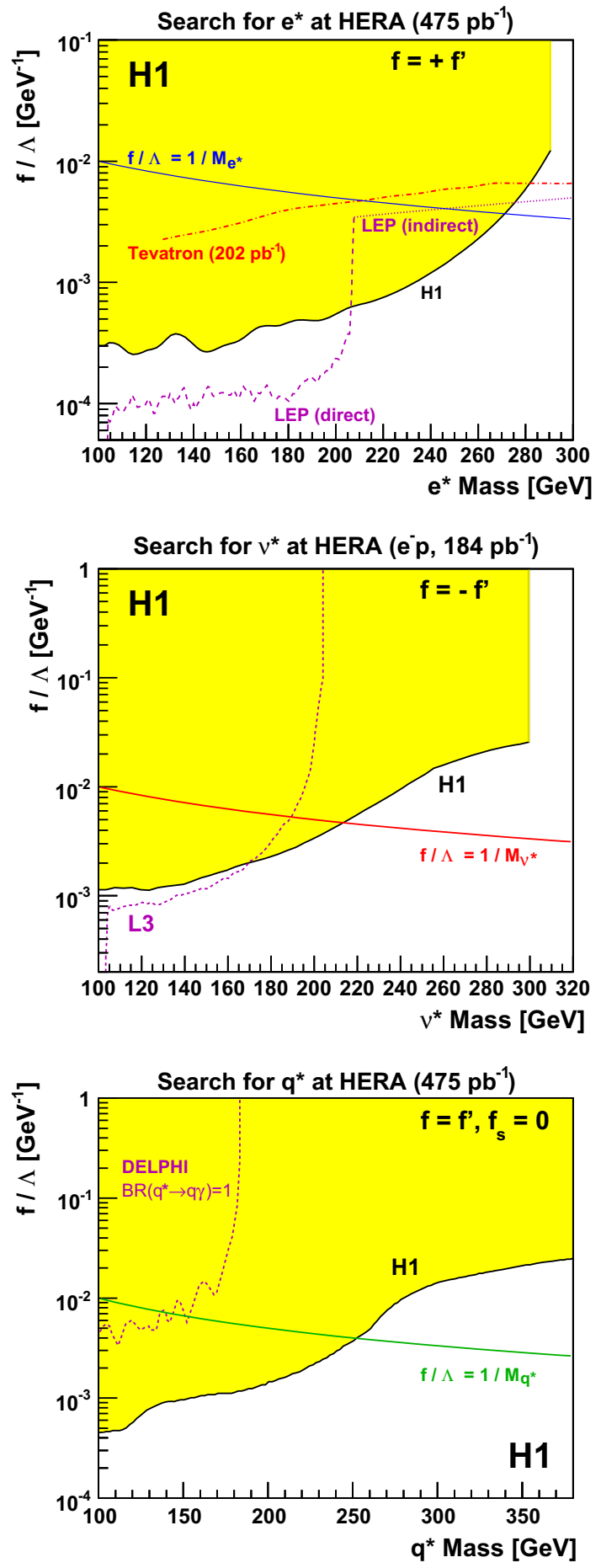

Fig. 51 Exclusion limits at $95 \% \mathrm{CL}$ on the ratio $f / \Lambda$ as a function of $M_{f^{*}}$, for excited electrons (top), excited neutrinos (middle) and excited quarks (bottom) with the assumptions given in the text and on the plot. Values of $f / \Lambda$ above the curves are excluded. Limits from the LEP and Tevatron colliders are also indicated

and then subsequently decay to a number of different finalstate topologies which may be investigated at HERA [257]. Searches for such final states have been performed by both
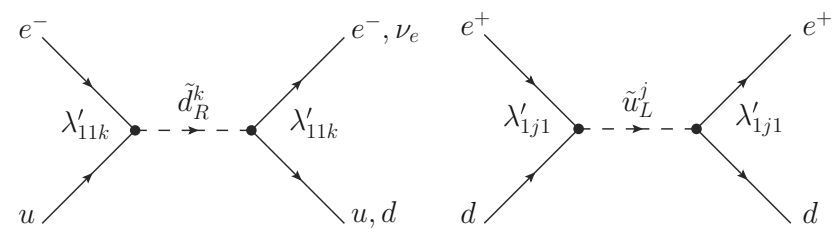

Fig. 52 Feynman diagrams for the single resonant s-channel production of right-handed down-type squarks in $e^{-} p$ collisions (left) and left-handed up-type squarks in $e^{+} p$ collisions (right) with subsequent decays into SM particles via Yukawa couplings $\lambda_{11 k}^{\prime}$ or $\lambda_{1 j 1}^{\prime}$, respectively. The right-handed down-type squarks can decay either into $e^{-} u$ or $v_{e} d$, while the left-handed up-type squarks decay into $e^{+} d$ only

H1 [258] and ZEUS [259] using their HERA I data sets. In this review we focus on the most recent H1 analysis [260], which employs the full HERA data set, corresponding to an integrated luminosity $183 \mathrm{pb}^{-1}$ for $e^{-} p$ collisions and $255 \mathrm{pb}^{-1}$ for $e^{+} p$ collisions.

Feynman diagrams of the resonant production and decay of squarks at HERA via $R_{p}$ couplings $\lambda_{1 j k}^{\prime}$ are shown in Fig. 52. For simplicity, it is assumed here that one of the $\lambda_{1 j k}^{\prime}$ couplings dominates over all the other trilinear $R_{p}$ couplings. At the high values of Bjorken- $x$ which are required to produce squarks of significant mass, the valence quarks are the dominant contribution to the proton PDFs. Therefore, $e^{-} p$ scattering gives sensitivity to the couplings $\lambda_{11 k}^{\prime}(k=1,2,3)$, which dominate the production of $\tilde{d}_{R}$-type squarks (i.e. the superpartners $\tilde{d}_{R}, \tilde{s}_{R}$ and $\tilde{b}_{R}$ of down-type quarks). Conversely, $e^{+} p$ scattering provides sensitivity to the couplings $\lambda_{1 j 1}^{\prime}(j=1,2,3)$, which dominate the production of $\tilde{u}_{L^{-}}$ type squarks (i.e. the superpartners $\tilde{u}_{L}, \tilde{c}_{L}$ and $\tilde{t}_{L}$ of up-type quarks). Due to the larger $u$-quark density in the proton at large $x$ with respect to the $d$-quark density, larger production cross sections are expected in $e^{-} p$ interactions for identical couplings and squark masses.

All sparticles are unstable in $R_{p}$ SUSY and squarks can decay directly via the Yukawa coupling $\lambda_{i j k}^{\prime}$ into SM fermions. The $\tilde{d}_{R}^{k}$-type $(k=1,2,3)$ squarks can decay via the coupling $\lambda_{11 k}^{\prime}$ either into $e^{-} u$ or $v_{e} d$, while the $\tilde{u}_{L}^{j}$-type $(j=1,2,3)$ squarks decay via the coupling $\lambda_{1 j 1}^{\prime}$ into $e^{+} d$ only, as illustrated in Fig. 52. Squarks may also decay via $R_{p}$ conserving gauge couplings with subsequent $R_{p}$ decay into SM particles via the Yukawa coupling $\lambda_{1 j k}^{\prime}$. In this case, the $\tilde{u}_{L}$-type squarks can undergo a gauge decay into states involving a neutralino $\chi_{i}^{0}(i=1,2,3,4)$, a chargino $\chi_{i}^{+}(i=1,2)$ or a gluino $\tilde{g}$. However, $\tilde{d}_{R}$-type squarks mainly decay to $\chi_{i}^{0}$ or $\tilde{g}$ and decays into charginos are suppressed [257]. The resulting final states observed in the detector from such cascade decays may contain multiple leptons and jets as well as missing transverse momentum. Such a cascade decay is illustrated in Fig. 53, where a $\tilde{d}_{R}^{k}$-type squark 


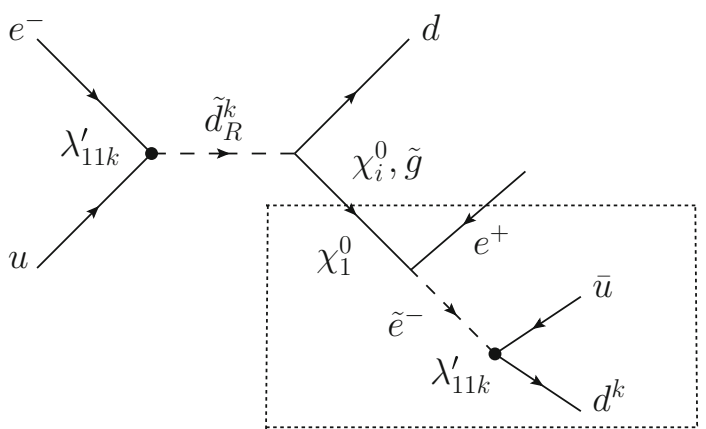

Fig. 53 Feynman diagram for squark production and subsequent cascade decay via gauginos, shown here for the case of right-handed downtype squarks with subsequent $R_{p}$ slepton decay into SM fermions via Yukawa couplings $\lambda_{11 k}^{\prime}$. Decays of down-type squarks to charginos are suppressed, and the $\tilde{d}_{R}$ decays to either $\chi_{i}^{0}$ (where $i=1,2,3,4$ ) or $\tilde{g}$

decays via a neutralino $\chi_{1}^{0}$ to a selectron-electron pair, and finally the selectron decays to SM fermions.

Squark signal events are simulated using cross sections obtained in the narrow width approximation from the leading-order amplitudes in leptoquark production [115], adjusted to NLO QCD using multiplicative correction factors [261]. The parton densities are evaluated at the hard scale $M_{\tilde{q}}^{2}$. A dedicated MC simulation is performed for each of the signal topologies: for the direct lepton-quark decay channels $e q$ and $v q$ shown in Fig. 52, events are generated using LEGO [262], whereas for the gauge decays of squarks such as the one in Fig. 53, events are generated using SUSYGEN3 [263]. To allow for a model-independent interpretation of the results, the squark-decay processes are simulated for a wide range of sparticle masses. Further details on the signal event simulation can be found in the $\mathrm{H} 1$ publication [260].

As explained above, the squark decays in this analysis can produce a large variety of final states, which are classified [258] into event topologies, or channels, to be examined. This classification relies on the number of isolated electrons, muons and hadronic jets in the final state, as well as on the presence of missing transverse momentum, indicating undetected neutrinos. A list of channels investigated in this analysis can be found in Table 14. The channels labelled $e q$ and $v q$ are the squark-decay modes that proceed directly via $R_{p}$ couplings resulting in event topologies with an isolated electron or neutrino and a single jet. The remaining channels result from the gauge decays of the squark and are characterised by final states with more than one jet, ("multi-jet", $M J$ ) with additional leptons. The $e M J$ and $v M J$ channels involve one or two gauginos in the decay cascade. In the $e M J$ channel, an electron or positron can be found in the final state and a distinction may be made with respect to the incident beam lepton charge and therefore, two discrete channels with the "right" (same) sign lepton charge $e M J(\mathrm{RC})$ and "wrong" (opposite) sign lepton charge $e M J(\mathrm{WC})$ are formed. Chan- nels with an electron or neutrino and a further charged lepton are denoted $e e M J, e \mu M J$ and $e \nu M J, \nu \mu M J$, respectively, and necessarily involve two gauginos.

Several SM processes may mimic the final states produced by squark decays and therefore a standard selection of MC generators is employed to compare the observed data events to the prediction from the SM. NC DIS events are simulated using RAPGAP and both direct and resolved photoproduction of jets, as well as prompt photon production, are simulated using PYTHIA. Inclusive CC DIS events are simulated using DJANGOH. The leading-order MC prediction of processes with two or more high transverse momentum jets in NC DIS, CC DIS and photoproduction is scaled by a factor of 1.2 to account for the incomplete description of higher orders in the MC generators [229,264]. Additional, smaller contributions from single- $W$ boson production and lepton pair production are modelled using EPVEC and GRAPE, respectively.

Each of the final states listed in Table 14 is analysed separately using a dedicated event selection [260]. These selections are based on the identification of high $P_{T}$ electrons, muons, jets, as well as missing transverse momentum. The final state of a squark decaying into an electron and a high $P_{T}$ jet is identical to that from a NC DIS event at high $x$ and $Q^{2}$, and therefore the event selection in the $e q$ channel closely resembles the selection described in Sect. 8: An isolated electron is required in the event with $P_{T}^{e}>16 \mathrm{GeV}$ in the region $5^{\circ}<\theta_{e}<145^{\circ}$, and the event must be in the kinematic phase space $Q_{e}^{2}>2500 \mathrm{GeV}^{2}, y_{e}<0.9,40 \mathrm{GeV}$ $<\delta<70 \mathrm{GeV}$ and $P_{T}^{\text {miss }}<15 \mathrm{GeV}$. The squark mass is reconstructed as $M_{e}=\sqrt{x_{e} s}$ and a mass-dependent $y_{e}$ cut is added to separate the NC background from the signal [260].

Squarks decaying into a neutrino and a high $P_{T}$ jet lead to the same signature as CC DIS events with high missing transverse momentum, and so the event selection in the $v q$ channel is accordingly based on the analysis of such events. Events with a neutrino are selected by requiring $P_{T}^{\text {miss }}>30$ and $\delta<50 \mathrm{GeV}$ and the phase space is restricted to $Q_{h}^{2}>$ $2500 \mathrm{GeV}^{2}$ and $y_{h}<0.9$. Similarly to the $e q$ channel, a $y_{h}$ cut dependent on the reconstructed mass $M_{h}=\sqrt{x_{h} s}$ is applied. The $v q$ channel is not relevant for $e^{+} p$ data since the $\tilde{u}_{L}$-type squarks produced in $e^{+} p$ do not undergo this decay.

Squarks decaying via neutralinos or charginos are expected to have a higher multiplicity of jets and leptons in the final state and their signatures correspond to final states detectable in higher-order NC DIS processes. Squark decays with single or multiple neutrinos produced via neutralino or chargino decays can result in final states similar to that of higherorder CC DIS processes. The remaining channels can therefore be divided into two groups, for which common preselections are employed: on the one side electron-multi-jet and electron-lepton-multi-jet final states and on the other side secondly neutrino-multi-jet and neutrino-muon-multi- 
Table 14 Summary of the observed and predicted event yields for the different decay channels considered in $\mathrm{H} 1$ search for squarks in $R_{p}$ SUSY, in the $e^{-} p$ and $e^{+} p$ data. The total uncertainties on the $\mathrm{SM}$ prediction is determined by adding the effects of all model and experimental systematic uncertainties in quadrature. The range of signal efficiencies is also given for each channel, for squark masses ranging from 100 to $290 \mathrm{GeV}$ and gaugino masses ranging from $30 \mathrm{GeV}$ up to the squark mass

\begin{tabular}{|c|c|c|c|c|c|}
\hline \multirow[t]{2}{*}{ Channel } & \multicolumn{2}{|c|}{$e^{-} p$ collisions, $\mathcal{L}=183 \mathrm{pb}^{-1}$} & \multicolumn{2}{|c|}{$e^{+} p$ collisions, $\mathcal{L}=255 \mathrm{pb}^{-1}$} & \multirow[t]{2}{*}{ Signal efficiency $(\%)$} \\
\hline & Data & Total SM & Data & Total SM & \\
\hline$e q$ & 3121 & $3215 \pm 336$ & 2946 & $2899 \pm 302$ & $30-40$ \\
\hline$v q$ & 2858 & $2983 \pm 358$ & - & - & $50-60$ \\
\hline$e M J(\mathrm{RC})$ & 147 & $158.3 \pm 23.9$ & 140 & $146.0 \pm 21.4$ & $10-40$ \\
\hline$e M J(\mathrm{WC})$ & 0 & $1.3 \pm 0.3$ & 1 & $0.6 \pm 0.4$ & $5-20$ \\
\hline$e e M J$ & 0 & $1.5 \pm 0.5$ & 2 & $1.7 \pm 0.5$ & $5-35$ \\
\hline$e \mu M J$ & 0 & $0.03 \pm 0.02$ & 0 & $0.03 \pm 0.03$ & $5-15$ \\
\hline$e v M J$ & 3 & $5.6 \pm 1.2$ & 5 & $8.2 \pm 2.0$ & $5-40$ \\
\hline$v M J$ & 204 & $235.5 \pm 63.3$ & 113 & $134.0 \pm 33.8$ & $5-15$ \\
\hline$v \mu M J$ & 0 & $0.04 \pm 0.02$ & 0 & $0.06 \pm 0.03$ & $5-20$ \\
\hline
\end{tabular}

jet final states. Further cuts are then applied depending on the number and flavour of the leptons in the event, as well as the charge, to separate the $e M J(R C)$ and $e M J(W C)$ channels. The event selections are optimised to maximise the signal efficiency in each channel and are described in the publication [260]. For each selected event a squark mass $M_{\mathrm{rec}}=\sqrt{4 E_{e}^{0}\left(\sum E_{i}-E_{e}^{0}\right)}$ is calculated, where the sum includes the energies of the reconstructed electrons, muons and jets with $P_{T}^{\text {jet }}>5 \mathrm{GeV}$ in the event, in addition to the neutrino in the case of the $\nu M J$ and $\nu \mu M J$ channel.

The number of events observed in the data in each channel is shown in Table 14 compared to the SM prediction, where good agreement is observed in all channels. Of the gauge decays, only the $e M J(R C)$ and $v M J$ channels have significant event yields. The invariant mass distributions of some of the most populous squark-decay channels are displayed in Fig. 54, where the data are in good agreement with the SM expectation. As no significant deviation from the SM is observed, all analysis channels are combined to set constraints on various supersymmetric models as described in the following. Mass-dependent exclusion limits are obtained [260] on the production of squarks parameterised by the strength of the $R_{p}$ couplings $\lambda_{1 j 1}^{\prime}$ and $\lambda_{11 k}^{\prime}$.

For the interpretation of the results a version of the Minimal Supersymmetric Standard Model (MSSM) is considered where the masses of the neutralinos, charginos and gluinos are determined via the usual SUSY parameters: the Higgs mass term $\mu$, which mixes the Higgs super-fields; the SUSY soft-breaking mass parameter $M_{2}$; and the ratio of the vacuum expectation values of the two neutral scalar Higgs fields $\tan \beta[138,139]$. The parameters are defined at the electroweak scale and the lightest supersymmetric particle (LSP) is the neutralino $\chi_{1}^{0}$. Slepton masses $M_{\tilde{l}}$ are fixed at $90 \mathrm{GeV}$. Exclusion limits are calculated for two scenarios with $\tan \beta=2$ : a photino-like neutralino $(\mu=-200 \mathrm{GeV}$, $\left.M_{2}=80 \mathrm{GeV}\right)$ and a zino-like neutralino $(\mu=200 \mathrm{GeV}$, $\left.M_{2}=150 \mathrm{GeV}\right)$. A combination of both scenarios is also achieved by performing a full parameter scan, where the parameters $M_{2}$ and $\mu$ are varied in the range $70 \mathrm{GeV}<M_{2}<$ $350 \mathrm{GeV}$ and $-300 \mathrm{GeV}<\mu<300 \mathrm{GeV}$ for $\tan \beta=6$. As an example, exclusion limits at $95 \% \mathrm{CL}$ from the parameter scan for the $\lambda_{131}^{\prime}$ coupling as a function of squark mass using the full $\mathrm{H} 1 e^{+} p$ data are shown in Fig. 55 (left), compared to the previous H1 limit [258] and an indirect limit from atomic parity violation [265]. In the parameter space considered in the analysis, Yukawa couplings of electromagnetic strength, $\lambda_{1 j 1}^{\prime}$ or $\lambda_{11 k}^{\prime}=\sqrt{4 \pi \alpha_{\mathrm{em}}}=0.3$, are excluded up to masses of $275 \mathrm{GeV}$ at $95 \% \mathrm{CL}$ for up-type squarks and up to masses of $290 \mathrm{GeV}$ for down-type squarks.

Constraints are also obtained on the Minimal Supergravity Model (mSUGRA) [266-269], which is a model that assumes gauge coupling unification and radiative electroweak symmetry breaking with the choice of 5 parameters: the common mass of scalar sparticles $m_{0}$; the common mass of fermionic sparticles $m_{1 / 2}$; the common trilinear coupling $A_{0}$; the sign of the Higgs mixing parameter $\mu$ and $\tan \beta$ as defined above. The masses of squarks, sleptons and gauginos as well as the branching ratios in the analysis channels are determined by the parameter set for given values of the couplings $\lambda_{11 k}^{\prime}$ and $\lambda_{1 j 1}^{\prime}$. $A_{0}$ enters only marginally in the interpretation and is set to zero. The parameter $\mu$ is taken with negative sign. Figure 55 (right) shows example exclusion limits at $95 \%$ in the $m_{0}, m_{1 / 2}$ plane assuming $\lambda_{1 j 1}^{\prime}=0.3$ and $\tan \beta=6$, obtained using the full $\mathrm{H} 1 e^{+} p$ data. The excluded region typically covers masses of $m(\tilde{u})=275$ and $m(\tilde{t})=270 \mathrm{GeV}$, as indicated in the figures. A constraint from the $\mathrm{D} \emptyset$ exper- 

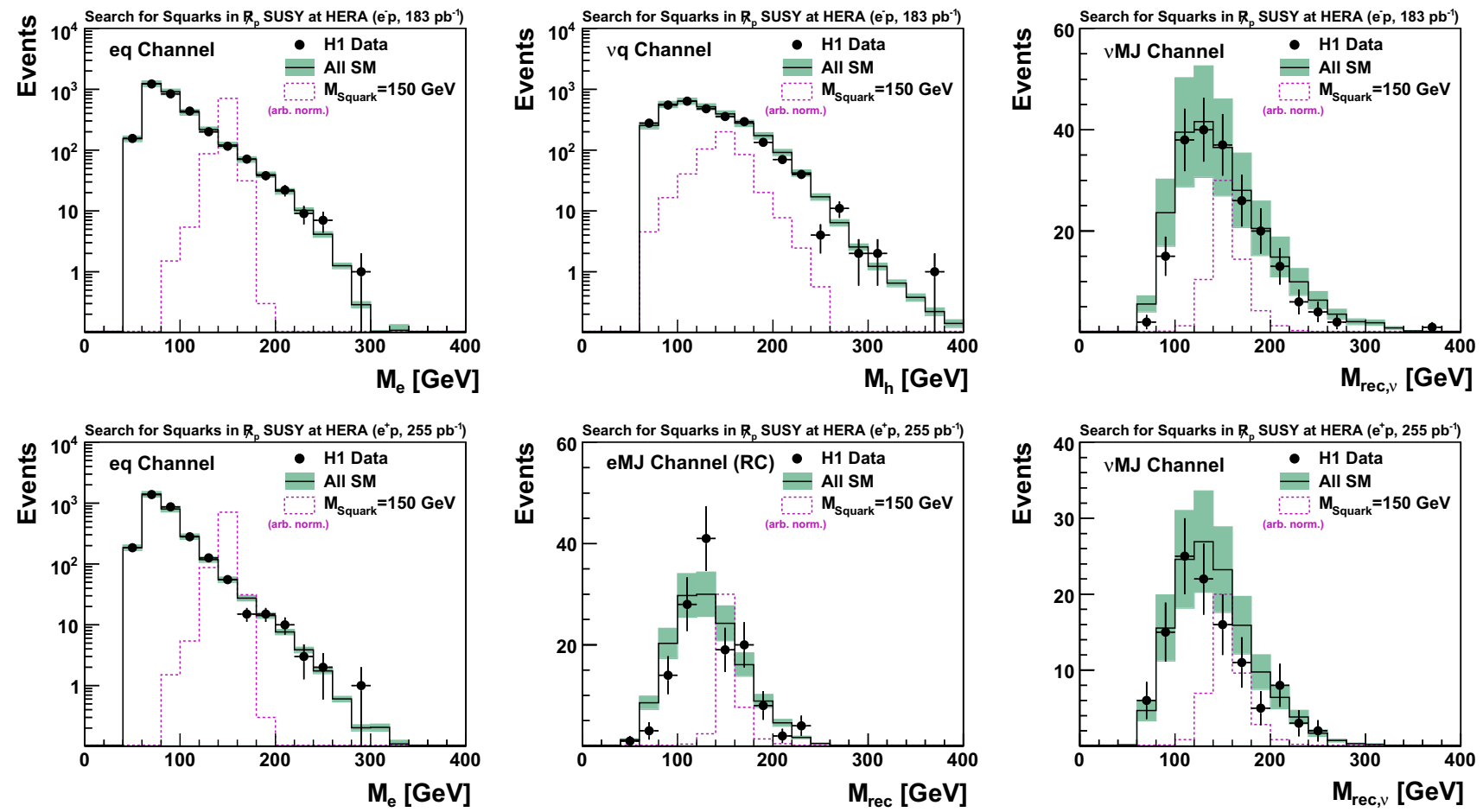

Fig. 54 Selected reconstructed invariant mass distributions from the H1 search for squarks in the $e^{-} p$ data (top row) and $e^{+} p$ data (bottom row). The data (points) are compared to SM MC predictions. The error band represents all model and experimental systematic uncertainties on the SM prediction (solid histogram) added in quadrature. The dashed histogram indicates the signal from a squark with $M_{\tilde{q}}=150 \mathrm{GeV}$ with arbitrary normalisation

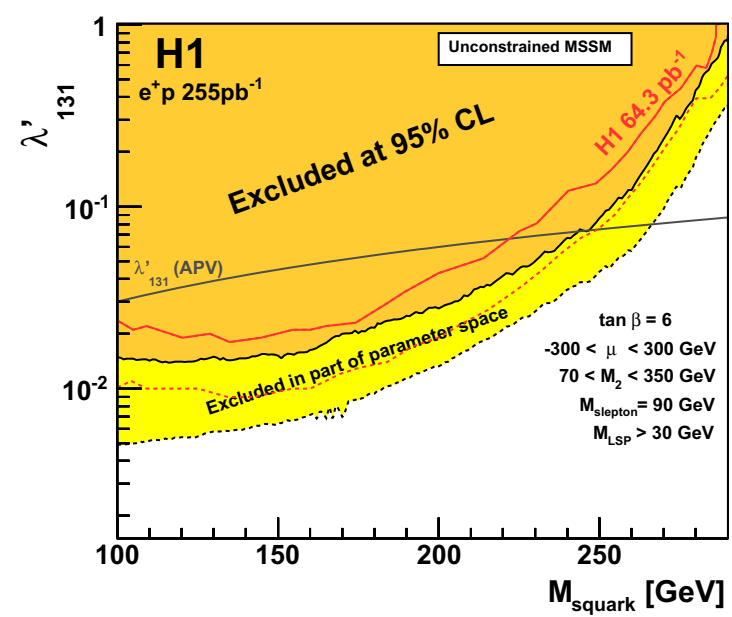

Fig. 55 Left exclusion limits at the $95 \% \mathrm{CL}$ on $\lambda_{131}^{\prime}$ as a function of the squark mass from a scan of the MSSM parameter space as indicated in the figure. The dark filled region indicates values of the coupling $\lambda_{131}^{\prime}$ excluded in all investigated scenarios whereas the light filled region is excluded only in part of the parameter space. An indirect limit from atomic parity violation (APV) is also shown, as well as the limit from the previous $\mathrm{H} 1$ analysis. Right exclusion limits at the

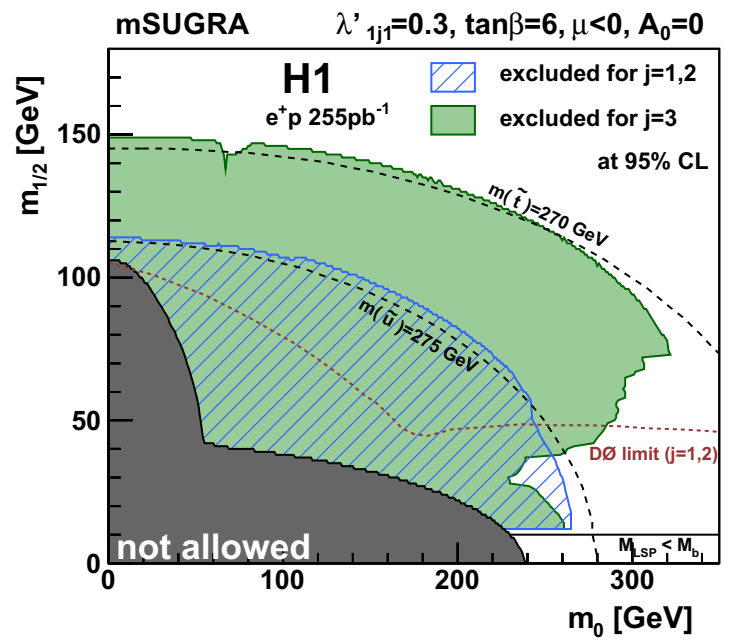

95\% CL in the $m_{0}, m_{1 / 2}$ plane in the mSUGRA parameter space indicated in the figure for $j=1,2$ (striped region) and $j=3$ (light filled region). Curves of constant squark mass are illustrated for $m(\tilde{u})=275$ and $m(\tilde{t})=270 \mathrm{GeV}$. A constraint obtained by the $\mathrm{D} \emptyset$ experiment at the Tevatron is also indicated. The dark filled region labelled as "not allowed" indicates where no radiative electroweak symmetry breaking solution is possible or where the LSP is a sfermion 

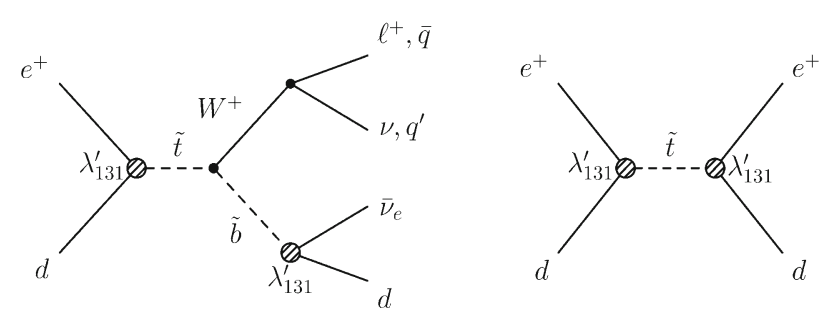

Fig. 56 Lowest-order $s$-channel diagram for $R_{p}$ stop production at HERA followed by (left) the bosonic decay of the stop and (right) the $R_{p}$ decay of the stop

iment [270] at the Tevatron using di-electron events is also indicated, where the region excluded by $\mathrm{H} 1$ is considerably larger.

\subsection{Search for bosonic stop decays in $\boldsymbol{R}$-parity violating supersymmetry}

In most SUSY models the third generation squarks, namely $\operatorname{stop}(\tilde{t})$ and sbottom $(\tilde{b})$, are the lightest squarks. If the sbottom mass is smaller than the stop mass, $M_{\tilde{b}}<M_{\tilde{t}}$, a stop quark resonantly produced in $e q$-fusion at HERA via the $R_{p} \quad$ coupling $\lambda_{131}^{\prime}$ may then decay bosonically, providing a SUSY scenario and final states complementary to the $R_{p}$ squark production described in Sect. 17.1. In this scenario, the only possible decay modes are $\tilde{t} \rightarrow \tilde{b} W$ with $W \rightarrow f \bar{f}^{\prime}$ and $R_{p} \quad$ sbottom decay into SM particles, $\tilde{b} \rightarrow \bar{v}_{e} d$. In addition, the $R_{p}$ decay of the stop into SM fermions, $\tilde{t} \rightarrow e^{+} d$, a more general version of which is described in the previous section, also contributes. Feynman diagrams of these two processes are presented in Fig. 56. The diagram where the $W$ decays leptonically is particularly interesting, as it results in a final state similar to that in the analysis of events with isolated leptons and missing transverse momentum presented in Sect. 12, which has provided hints of physics beyond the SM.

The analysis presented below uses data collected with the $\mathrm{H} 1$ detector in $e^{+} p$ scattering during the HERA I period, corresponding to an integrated luminosity of $106 \mathrm{pb}^{-1}$ [271]. Simulation of SUSY signal events is done using SUSYGEN3 [263], which relies on the LO amplitudes for $e d \rightarrow \tilde{b} W[272]$. The parton densities are taken from the CTEQ5L parameterisation and evaluated at the scale of the stop mass, $M_{\tilde{t}}$. The various SM background contributions are estimated using the same generators as described in Sect. 17.1.

The bosonic stop decay leads to three different final-state topologies, as illustrated in Fig. 56 (left). If the $W$ decays into leptons, the signature is a jet, a lepton (electron or muon $)^{16}$ and missing transverse momentum $\left(j e P_{T}^{\text {miss }}\right.$ chan-

\footnotetext{
16 The $W$ decay into $\nu_{\tau} \tau$, where $\tau \rightarrow$ hadrons $+\nu$, is not investigated.
}

nel and $j \mu P_{T}^{\text {miss }}$ channels). The selection criterion in these channels closely resembles those used in the $\mathrm{H} 1$ search for events with isolated leptons and missing transverse momentum based on the HERA I data [192], with the additional requirement of a jet with $P_{T}^{\text {jet }}>10 \mathrm{GeV}$ within the angular range $7^{\circ}<\theta_{\text {jet }}<140^{\circ}$. If the $W$ decays into hadrons the event signature is the presence of three jets and missing transverse momentum $\left(j \mathrm{jj} P_{T}^{\text {miss }}\right.$ channel). Events with three jets with $P_{T}^{\text {jet1 }}>20, P_{T}^{\text {jet2 }}>15$ and $P_{T}^{\text {jet3 }}>10 \mathrm{GeV}$ are selected, each with polar angle $7^{\circ}<\theta_{\text {jet }}<140^{\circ}$. A total missing transverse momentum $P_{T}^{\text {miss }}>25 \mathrm{GeV}$ is also required. In the analysis of both the leptonic and hadronic $W$-decay channels a cut on the inelasticity $y$ is employed to separate the SM background from the stop signal [271]. For stop and sbottom masses $M_{\tilde{t}} \approx M_{\tilde{b}}+M_{W}$, the $R_{p}$ decay $\tilde{t} \rightarrow e d$, as illustrated in Fig. 56 (right), becomes dominant. These events are selected using criteria similar, though not identical, to that presented in Sect. 17.1.

The number of events observed in the data in each channel is shown in Table 15, compared to the SM prediction. The dominant contribution to the SM expectation in the $j e P_{T}^{\text {miss }}$ and $j \mu P_{T}^{\text {miss }}$ channels arises from the production of real $W$ bosons, which is also given in Table 15. The main SM contribution in the $j j j P_{T}^{\text {miss }}(e d)$ channel is due to CC (NC) DIS events. A good agreement is observed in all channels except in the $j \mu P_{T}^{\text {miss }}$ channel, where 8 events are observed in the data compared to a SM expectation of $2.69 \pm 0.47$.

Assuming the presence of a stop of mass $M_{\tilde{t}}$ decaying bosonically, the observed event yields are used to determine the allowed range for a stop production cross section $\sigma_{\tilde{t}}$, and to examine the compatibility of the different decay modes. The calculation takes into account the signal efficiency, the $\tilde{t}$ and $W$ branching ratios $B R_{\tilde{t} \rightarrow \tilde{b} W} \cdot B R_{W \rightarrow f \bar{f}^{\prime}}$ and the relative integrated luminosities of the HERA I data sets taken at different centre of mass energies [271]. The bands in Fig. 57 (left) represent the allowed cross section regions $\sigma_{\tilde{t}} \pm \Delta \sigma_{\tilde{t}}$ for all bosonic decay channels. It can be seen that the stop interpretation of the excess seen in the $j \mu P_{T}^{\text {miss }}$ channel is not supported by the other decay modes.

As no stop signal is observed, exclusion limits on the stop production cross section are derived at the $95 \% \mathrm{CL}$ in the framework of the MSSM $[138,139]$ using a modified frequentist approach based on likelihood ratios [178]. A scan of the SUSY parameter space is performed, to systematically investigate the dependence of the sensitivity on the MSSM parameters. The SUSY soft-breaking mass parameter $M_{2}$ is set to $1000 \mathrm{GeV}$ and the Higgs mass term is restricted to $400<\mu<1000 \mathrm{GeV}$, which ensures that the gaugino masses are large. The mixing angles $\theta_{\tilde{t}}$ and $\theta_{\tilde{b}}$ are allowed to vary between $0.6 \mathrm{rad}$ and $1.2 \mathrm{rad}$. An example of the limit 
Table 15 Summary of the observed and predicted event yields for the various stop-decay channels in the $\mathrm{H} 1$ analysis. The uncertainty on the SM predictions includes model and experimental systematic uncertain- ties added in quadrature. For the $j e P_{T}^{\text {miss }}$ and $j \mu P_{T}^{\text {miss }}$ channels the $W$ production component of the SM is given in the last column

\begin{tabular}{lccc}
\hline H1 search for bosonic stop decays in $R_{p}$-violating supersymmetry $\left(e^{ \pm} p\right.$ collisions, $\left.\mathcal{L}=106 \mathrm{pb}^{-1}\right)$ \\
\hline Channel & Data & Total SM & $W$ production \\
\hline$j e P_{T}^{\text {miss }}$ & 3 & $3.84 \pm 0.92$ & $2.55 \pm 0.41$ \\
$j \mu P_{T}^{\text {miss }}$ & 8 & $2.69 \pm 0.47$ & $1.93 \pm 0.31$ \\
$j j j P_{T}^{\text {miss }}$ & 5 & $6.24 \pm 1.74$ & - \\
$e d$ & 1100 & $1120 \pm 131$ & - \\
\hline
\end{tabular}

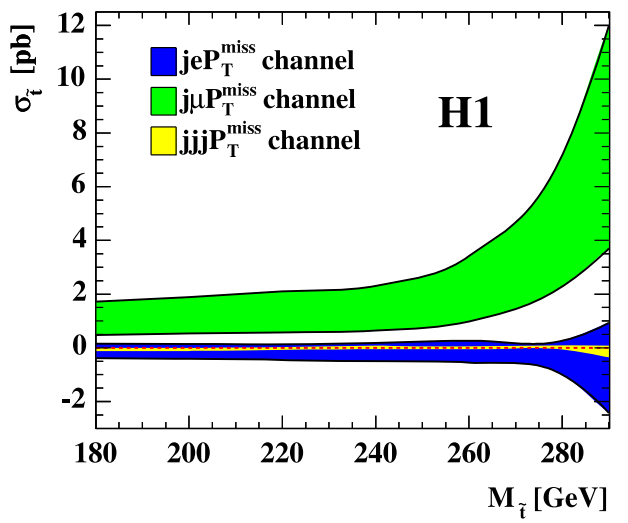

Fig. 57 Left bands representing the allowed stop cross section regions $\sigma_{\tilde{t}} \pm \Delta \sigma_{\tilde{f}}$ as a function of the stop mass as obtained from the analysis of each bosonic stop-decay channel. Right exclusion limits at the $95 \% \mathrm{CL}$ on the $R_{p}$ coupling $\lambda_{131}^{\prime}$ as a function of the stop mass for

projected in the $\left(M_{\tilde{t}}, \lambda_{131}^{\prime}\right)$ plane is shown in Fig. 57 (right), for $\tan \beta=10$ and $M_{\tilde{b}}=100 \mathrm{GeV}$. For $M_{\tilde{t}}=200 \mathrm{GeV}$, couplings $\lambda_{131}^{\prime} \gtrsim 0.03$ are ruled out and for $M_{\tilde{t}}=275 \mathrm{GeV}$ the allowed domain is $\lambda_{131}^{\prime} \lesssim 0.3$.

17.3 Search for light gravitinos in events with photons and missing transverse momentum

In Gauge Mediated Supersymmetry Breaking (GMSB) SUSY models, new "messenger" fields are introduced which couple to the source of supersymmetry breaking. The breaking is then transmitted to the SM fields and their superpartners by gauge interactions [273]. The gravitino, $\tilde{G}$, is the Lightest Supersymmetric Particle (LSP) and can be as light as $10^{-3} \mathrm{eV}$. The Next-to-Lightest Supersymmetric Particle (NLSP) is generally either the lightest neutralino $\tilde{\chi}_{1}^{0}$ or a slepton $\tilde{\ell}$, which decays to the stable gravitino via $\tilde{\chi}_{1}^{0} \rightarrow \gamma \tilde{G}$ or $\tilde{\ell} \rightarrow \ell \tilde{G}$. At HERA, the presence of the $R_{p}$ couplings $\lambda_{1 j 1}^{\prime}$ and $\lambda_{11 k}^{\prime}$ could lead to neutralino production in $e^{+} p$ and $e^{-} p$ collisions, respectively, via $t$-channel selectron exchange, as illustrated in Fig. 58. The hard scatter-

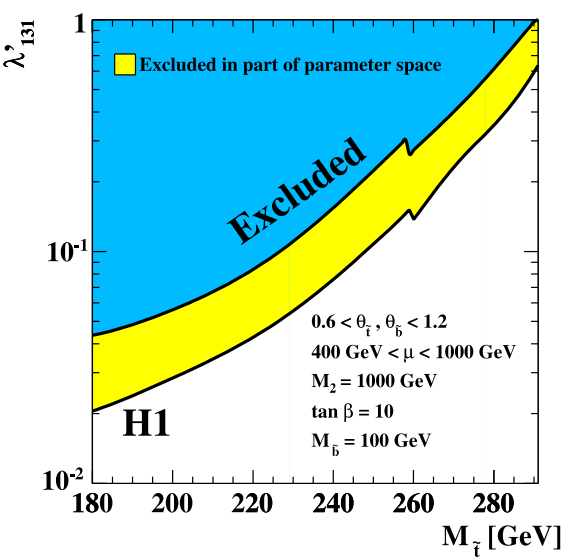

$M_{\tilde{b}}=100 \mathrm{GeV}$. The limits are derived from a scan of the MSSM parameter space described in the text. The two full curves indicate the regions excluded in all (dark) or part (light) of the parameter space investigated
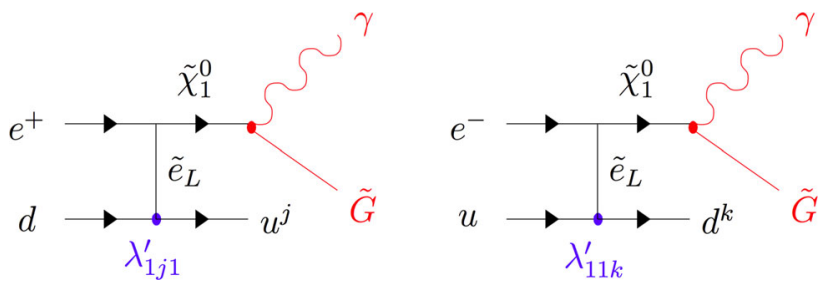

Fig. 58 Diagrams for neutralino production via $R_{p}$ selectron exchange in $e^{+} p$ (left) and $e^{-} p$ (right) scattering, with subsequent neutralino decay into a gravitino and a photon

ing process at large Bjorken- $x$ is dominated by the valence quarks in the proton, and therefore if the initial-state lepton is a positron (electron) the scatter involves a down (up) quark from the proton, as shown in the left (right) part of Fig. 58. For a given $R_{p}$ coupling, the $\tilde{\chi}_{1}^{0}$ production cross section for an initial-state electron is roughly a factor of two larger than that for an initial positron, reflecting the different parton densities for valence up and down quarks in the proton.

A search for $\mathbb{R}_{p}$ resonant single-neutralino production via $t$-channel selectron exchange, $e^{ \pm} q \rightarrow \tilde{\chi}_{1}^{0} q^{\prime}$ is performed by 
$\mathrm{H} 1$ using their HERA I data set taken at $\sqrt{s}=319 \mathrm{GeV}$, corresponding to an integrated luminosity of $64.3 \mathrm{pb}^{-1}$ for $e^{+} p$ collisions and $13.5 \mathrm{pb}^{-1}$ for $e^{-} p$ collisions [274]. It is assumed that the $\tilde{\chi}_{1}^{0}$ is the NLSP and that the decay $\tilde{\chi}_{1}^{0} \rightarrow \gamma \tilde{G}$ occurs with an unobservably small lifetime and dominates over $R_{p}$ neutralino decays. It is also assumed that one of the couplings $\lambda_{1 j 1}^{\prime}(j=1,2)$ or $\lambda_{11 k}^{\prime}(k=1,2,3)$ dominates. ${ }^{17}$ The process considered in this analysis is independent of the squark sector, and so is a complementary approach to those presented in Sects. 17.1 and 17.2.

The GMSB model [275] examined in the analysis is characterised by six new parameters in addition to those of the SM: the parameter $\Lambda$, which sets the overall mass scale for the SUSY particles; the mass of the messenger particles $M$; the number $f$ sets of messenger particles, $N$; the intrinsic SUSY breaking scale $\sqrt{F}$, which also determines the $\tilde{G}$ mass according to $m_{\tilde{G}} \simeq 2.5 \cdot F /(100 \mathrm{TeV})^{2} \mathrm{eV}$; the ratio of the Higgs vacuum expectation values $\tan \beta$; and the sign of the Higgs sector mixing parameter $\mu$. The signal topology is simulated using the SUSYGEN3 generator [263]. The parton densities are evaluated at the scale of the Mandelstam variable $-t$.

The final state resulting from the process $e^{ \pm} q \rightarrow \tilde{\chi}_{1}^{0} q^{\prime} \rightarrow$ $\gamma \tilde{G} q^{\prime}$ contains a photon, a jet originating from the scattered quark and missing transverse momentum due to the escaping gravitino. The SM background almost exclusively arises from radiative CC DIS, which features a jet, a photon and a neutrino in the final state and is modelled using DJANGOH. Smaller contributions from NC DIS, photoproduction and $W$ production are estimated using the DJANGOH, PYTHIA and EPVEC generators, respectively.

Events are selected with large missing transverse momentum determined from the calorimetric energy deposits, $P_{T}^{\text {calo }}>25 \mathrm{GeV}$. The events are also required to contain at least one hadronic jet in the range $10^{\circ}<\theta_{\text {jet }}<145^{\circ}$ and an identified photon in the LAr, both with transverse momenta greater than $5 \mathrm{GeV}$. Photons are identified using a shower shape analysis of energy deposits in the LAr calorimeter and for $\theta_{\gamma}>20^{\circ}$ an electromagnetic cluster is only accepted as a photon candidate if it is not associated with a charged track in the central tracking system. In addition, the photon must be isolated from any reconstructed jet with $P_{T}^{\text {jet }}>5 \mathrm{GeV}$. At this point in the selection, 12 events are observed in the data compared to a SM expectation of $11.5 \pm 1.5$, predominantly from radiative CC DIS.

Based on a study of this preselection, additional cuts are then applied to reduce the SM background, increasing the minimum photon transverse momentum $P_{T}^{\gamma}$ to $15 \mathrm{GeV}$ and requiring that the sum of the $E-P_{z}$ in the event is larger

\footnotetext{
$\overline{17}$ The coupling $\lambda_{131}^{\prime}$ is not studied in this analysis because the production of a top quark together with a neutralino is suppressed due to the high top quark mass.
}

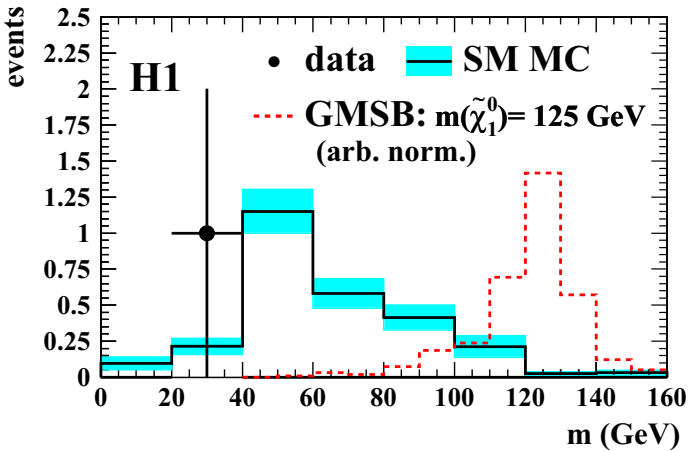

Fig. 59 Distribution of the invariant mass of the photon candidate and the reconstructed missing particle in the $\mathrm{H} 1$ search for light gravitinos in events with photons. The data (points) are compared with the SM prediction (solid histogram). The signal expected for a neutralino with a mass of $125 \mathrm{GeV}$ is shown with arbitrary normalisation (dashed histogram)

than $15 \mathrm{GeV}$ [274]. In the final selection no candidate event is found in the $e^{+} p$ data, compared to a SM prediction of $1.8 \pm 0.2$. In the $e^{-} p$ data sample, 1 candidate event is observed compared to a SM prediction of $1.1 \pm 0.2$. The SM expectation arises predominantly from CC DIS with small contributions from NC DIS and the production of $W$ and $Z$ bosons where the final-state electron is misidentified as a photon.

Assuming that the massless gravitino is the only noninteracting particle in the event, the gravitino kinematics are reconstructed by exploiting the conservation of transverse momentum and the constraint $\left(E-p_{z}\right)+\left(E_{\tilde{G}}-p_{z, \tilde{G}}\right)=2 E_{e}$. The four-vector of this particle is then added to that of the photon to reconstruct the invariant mass $m$ of the decaying neutralino. The data and the SM expectation for this distribution are shown in Fig. 59. From the simulation of the SUSY signal, also shown in Fig. 59, the mass resolution is determined to be around $10 \mathrm{GeV}$. The candidate event has a reconstructed invariant neutralino mass of $36 \pm 4 \mathrm{GeV}$.

As no significant deviation from the SM is observed, the results are used to derive constraints on GMSB models for different values of the $R_{p}$ coupling $\lambda_{1 j k}^{\prime}$ for fixed values of the SUSY parameters $\tan \beta, N$ and (sign) $\mu$. As an example, Fig. 60 displays excluded regions in the $m\left(\tilde{\chi}_{1}^{0}\right), m\left(\tilde{e}_{L}\right)-m\left(\tilde{\chi}_{1}^{0}\right)$ plane derived from the $\mathrm{H} 1 e^{+} p$ (left) and $e^{-} p$ (right) data for various values of $\lambda_{1 j k}^{\prime}$ in the parameter space indicated in the figure. It can be seen that for small mass differences between the neutralino and selectron, neutralino masses up to $112 \mathrm{GeV}$ are ruled out at $95 \% \mathrm{CL}$ for $R_{p}$ coupling $\lambda_{1 j k}^{\prime}=1$. Furthermore, for neutralino masses close to $55 \mathrm{GeV}, \lambda_{1 j 1}^{\prime}$ Yukawa couplings of electromagnetic strength are excluded. These are the only constraints from HERA on SUSY models independent of the squark sector. 

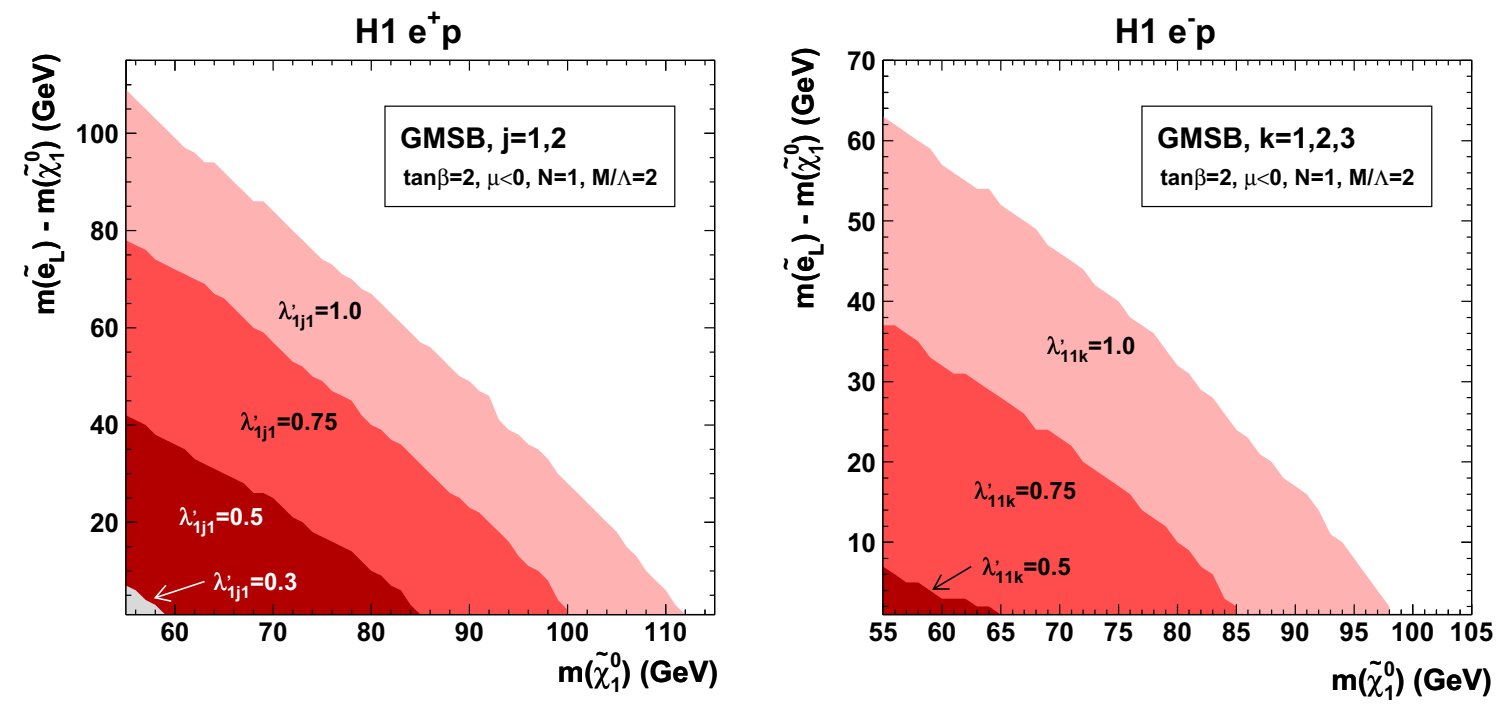

Fig. 60 Exclusion limits at the $95 \%$ CL in the $m\left(\tilde{\chi}_{1}^{0}\right), m\left(\tilde{e}_{L}\right)-m\left(\tilde{\chi}_{1}^{0}\right)$ plane from the H1 search for light gravitinos in events with photons, for various values of the coupling $\lambda_{1 j 1}^{\prime}(j=1,2)($ left $)$ and $\lambda_{11 k}^{\prime}(k=1,2,3)$ (right) in the GMSB SUSY parameter space indicated on the plot

\section{A direct search for stable magnetic monopoles}

The existence of magnetic monopoles is one of the open issues in modern physics and their discovery would allow a better explanation of some well established aspects of nature. The quantisation of the electric charge was explained by Dirac [276] by postulating the existence of particles with a magnetic charge, which shall be a multiple of the Dirac charge, $g_{D}$, given by

$\frac{g_{D} e}{\hbar c}=\frac{1}{2} \Rightarrow \frac{g_{D}}{e}=\frac{1}{2 \alpha_{\mathrm{e}}} \approx 68.5$

where $e$ is the elementary electric charge and $\alpha_{\mathrm{e}}$ is the fine-structure constant. With the presence of a magnetic monopole, considering the duality of Maxwell's equations the (very large) magnetic coupling can be expressed as

$\alpha_{\mathrm{m}}=\frac{g_{D}^{2}}{\hbar c}=\frac{1}{4 \alpha_{\mathrm{e}}}$.

The large value of this coupling constant prevents the use of perturbative field theory for a reliable calculation of the expected rates of processes involving magnetic monopoles. It also implies that the energy released by ionisation by a magnetic monopole is much larger than for minimum ionising electrically charged particles, as a magnetic monopole will effectively behave, in terms of ionisation energy loss, as a highly charged stable particle, with a charge $\approx 68.5$.

In addition to the Dirac argument, magnetic monopoles arise naturally in field theories unifying the fundamental forces, like string theory [277-279] and supersymmetric grand unified theories [280-283]. Although most of these theories tend to predict heavy monopoles, with masses higher than $10^{15} \mathrm{GeV}$, there are some grand unified scenarios [284286] in which mass values of the order of $10^{4} \mathrm{GeV}$ are allowed. Other approaches [287-291] also exist, in which a light monopole is allowed, and postulates on values of the classical radius of a monopole lead to estimates of a monopole mass of the order of tens of $\mathrm{GeV}$ [292].

One of the techniques that can be used in the direct search for magnetic monopoles is the search for the induction of a persistent current within a superconducting loop [293]. This approach is used by the H1 Collaboration in a direct search for magnetic monopoles [294], exploiting the idea that heavily ionising magnetic monopoles may stop in the beam pipe surrounding the interaction region of the $\mathrm{H} 1$ detector. Such monopoles, if stable, would then remain permanently trapped in the beam pipe, as the binding energy between the monopoles and the aluminium of the beam pipe is expected to be large, of the order of hundreds of keV [295]. The magnetic field of the trapped monopoles would induce a persistent current on a superconducting coil, after their complete passage through the coil. In contrast, a passage through the coil of material with no trapped monopole would induce no current, as the permanent dipole moment in the material would cancel in the passage of the material through the coil.

The aluminium beam pipe used in the search was in place in the H1 delector 1995-1997, during which time it was exposed to a luminosity of $62 \mathrm{pb}^{-1}$. The beam pipe had a diameter of $9 \mathrm{~cm}$ and thickness $1.7 \mathrm{~mm}$ in the range $-0.3<z<0.5 \mathrm{~m}$ and a diameter of $11 \mathrm{~cm}$ and thickness $2 \mathrm{~mm}$ in the range $0.5<z<2.0 \mathrm{~m}$. The analysis was performed by cutting the H1 beam pipe in long thin strips and then passing them through a superconducting coil 
Fig. 61 Method for the analysis of the $\mathrm{H} 1$ beam-pipe strips in the direct search for magnetic monopoles. The conveyor belt travelled in steps of typically $5 \mathrm{~cm}$ until the sample traversed completely the step the conveyor belt stopped for $1 \mathrm{~s}$ before the current in the superconducting coil (magnetometer current) was read to avoid the effects of eddy currents. The time for each step was typically $3 \mathrm{~s}$ superconducting coil. At each

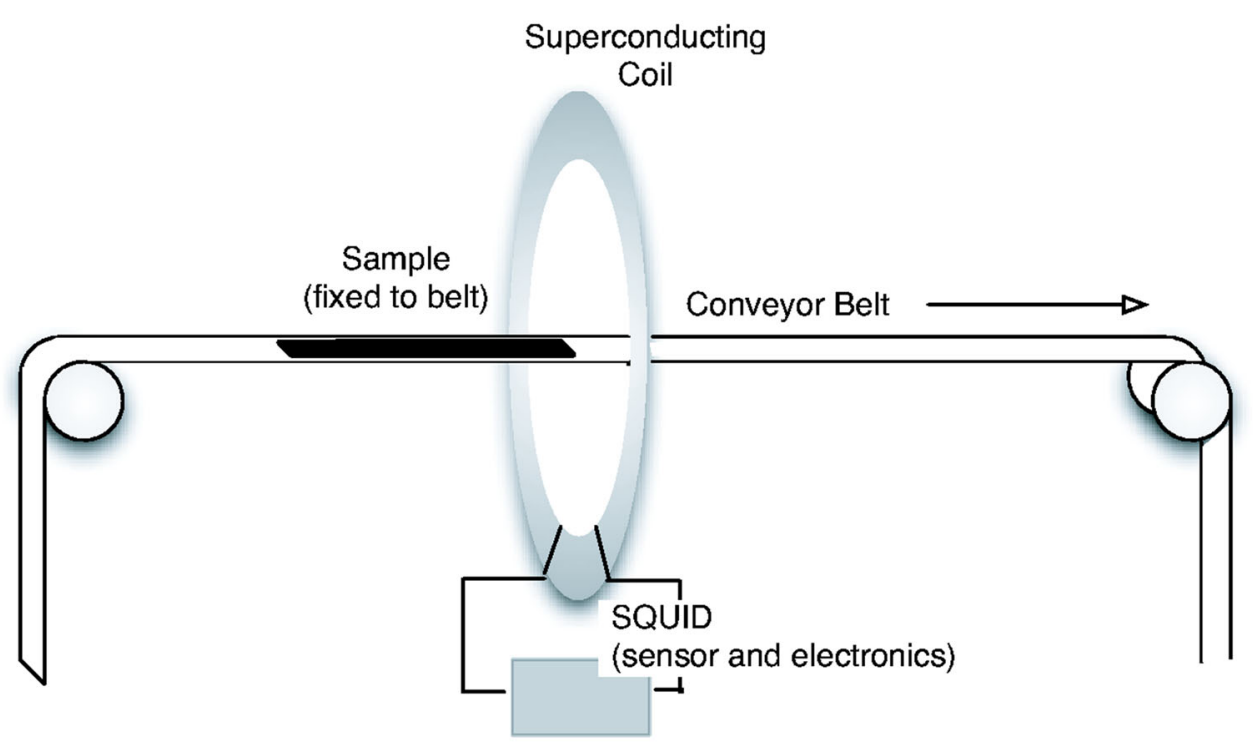

coupled to a Superconducting Quantum Mechanical Interference Device (SQUID), as shown in Fig. 61. This full length of the beam pipe, covering $-0.3<z<+2.0 \mathrm{~m}$, was cut into 45 longitudinal strips each of length on average of $573 \mathrm{~mm}(\sim 2 \mathrm{~mm}$ was lost in each cut). The central section $(-0.3<z<0.3 \mathrm{~m})$ was cut into 15 long strips of width $\sim 18 \mathrm{~mm}$, two of which were further divided into 32 short segments varying in length from 1 to $10 \mathrm{~cm}$. The downstream section $(0.3<z<2.0 \mathrm{~m})$ was divided into 3 longitudinal sections, each of which was cut into 10 long strips of width $\sim 32 \mathrm{~mm}$.

All the samples were passed along the axis of the $2 \mathrm{G}$ Enterprises type 760 magnetometer [296] hosted at the Southampton Oceanography Centre, in the United Kingdom. After each sample was passed through the coil, the current in the superconducting loop was measured, and the current induced by the sample estimated as the difference between the currents measured before and after its passage through the coil. This procedure was repeated many times for each sample in order to estimate the reproducibility of the results.

The sensitivity of the SQUID magnetometer to a magnetic monopole was assessed using a long, thin solenoid, as the magnetic field at the end of a long solenoid is similar to that produced by a magnetic monopole. A long solenoid can be considered as composed by two magnetic "pseudopoles, each of strength $g=N \cdot I \cdot S / g_{D}$, where $N$ is the number of turns per unit length, $I$ the current and $S$ the section area of the solenoid. The current and radius of the solenoid can be chosen to mimic the pole strength. The calibration was performed by passing a solenoid with different current through the magnetometer, measuring the induced current and subtracting the current induced at zero solenoid current. The current in the magnetometer following the passage of one end of the solenoid was found to increase linearly with the current in the solenoid.

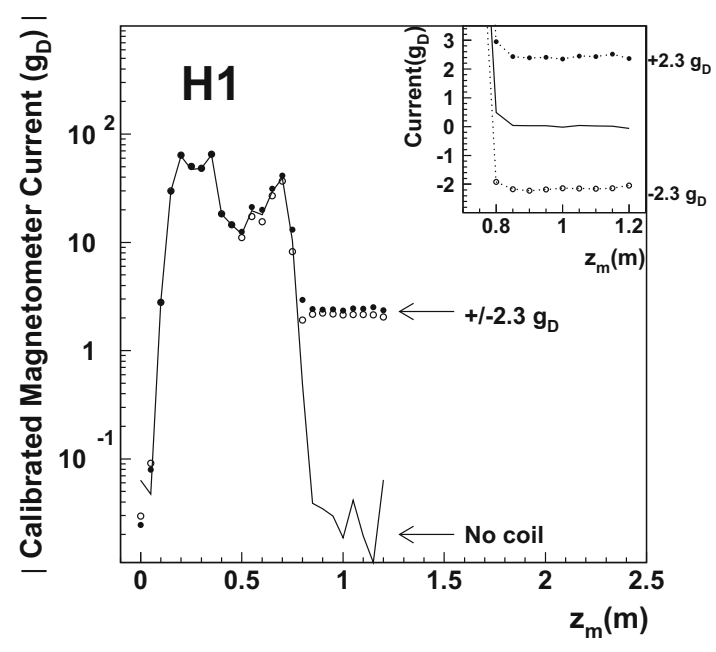

Fig. 62 The absolute value of the calibrated magnetometer current versus step position $\left(z_{m}\right)$ for a strip from the central beam-pipe region $(-0.3<z<0.3 \mathrm{~m})$. The solid line shows the measurements with the long strip alone. The closed (open) points show the measurements with the long strip together with the calibration solenoid excited to simulate a pole of strength $+2.3 g_{D}\left(-2.3 g_{D}\right)$. The inset shows the signed measurements of the calibrated magnetometer currents versus the step position for $z_{m}>0.8 \mathrm{~m}$ on a linear scale. The expected persistent currents for monopoles of strength $\pm 2.3 g_{D}$ are shown by the arrow on the logarithmic plot and by the numbers in the margin on the inset linear plot

To simulate the magnetometer behaviour at the passage of a magnetic monopole, the long solenoid was attached to a beam-pipe section and the two passed jointly through the coil, allowing the passage of only one end of the solenoid through the magnetometer. The results are shown in Fig. 62, where the passage of the beam-pipe strip alone is compared to the passage of the same strip attached to a solenoid with a "pseudopole" strength of $2.3 g_{D}$ and $-2.3 g_{D}$. A large structure is visible in the centre of the figure, and this is due to the 
magnetic field of the permanent magnetic dipole moments in the aluminium. A persistent current is induced when the pseudopoles are present; when they are absent, the large permanent dipole moment of the aluminium does not prevent the current to go back to zero. In the inset of the figure, the current measured as the strips leaves the magnetometer coil is shown. The values are equal and opposite, and equal to that measured with the calibration solenoid alone. This demonstrates the sensitivity of the apparatus to a magnetic monopole trapped in the beam-pipe strip.

The sample first analysed corresponds to the 15 strips cut from the region $-0.3<z<0.3 \mathrm{~m}$, out of which two were cut in 32 short samples. No persistent current was observed in the solenoid, and in the few cases in which it was observed, it disappeared in a subsequent measurement. The non-zero readout was therefore attributed to random jumps in the baseline of the magnetometer electronics. The conclusion was therefore that no magnetic monopole has been trapped in the central region of the beam pipe.

For the strips of the downstream beam pipe, some problems were encountered in the analysis. The permanent dipole moment of the downstream strips was found to be much larger than that of the strips of the central beam pipe, and gave readings in the magnetometer more than three orders of magnitude larger than those expected from a magnetic monopole. Moreover, fluctuations in the baseline level of the magnetometer up to $0.7 g_{D}$ were induced. To mitigate these effects, the strips were demagnetised in a low frequency magnetic field of initial intensity of $0.1 \mathrm{~T}$. This procedure was not expected to dislodge any potential magnetic monopoles trapped in the beam pipe, due to their large binding energy. Also in this case, no significant persistent current is observed and it is therefore concluded that no monopole of strength greater than $0.1 g_{D}$ had been trapped in the beam-pipe strips, which made up $93 \%$ of the total beam pipe, the rest being lost in the cutting procedure. Figure 63 shows a summary of the measurements performed on the whole sample.

As no magnetic monopole was observed, upper limits on the production cross section are determined. The cross section extraction needs the evaluation of the detector acceptance, therefore a model for the production is needed and two different models are considered. In each model, a monopoleantimonopole $(M \bar{M})$ pair was assumed to be produced in a photon-photon interaction. In the first model (model A), elastic production of a spin-0 monopole pair in the process $e^{+} p \rightarrow e^{+} M \bar{M} p$, through the interaction of a photon from the electron and a photon from the proton, was assumed. In the second model (model B), spin-1/2 monopoles were produced in the inelastic process $e^{+} p \rightarrow e^{+} M \bar{M} X$, through photon-photon fusion with a photon from the electron and one from a quark in the proton. Events for model A were generated using the program CompHEP [216], while a dedicated program was used for model B [294]. The generated

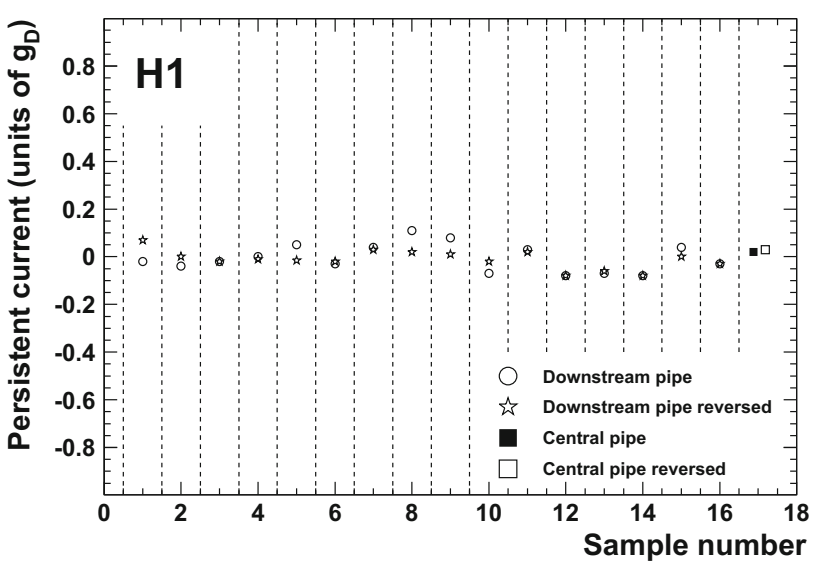

Fig. 63 The persistent currents in the long beam-pipe strips in units of $g_{D}$, after their passage through the magnetometer versus sample number, measured after the samples had been demagnetised (see text). Samples 1-16 consist of several long strips (usually two or three) from the downstream beam pipe bundled together. Sample 17 consists of the thirteen long strips of the central beam pipe bundled together

final-state particles were tracked in the $\mathrm{H} 1$ interaction region of the beam pipe, and if the range of the monopole in aluminium was lower than the thickness of the beam pipe, the monopole was assumed to stop there. The cross section upper limit was derived considering that no observation translates into a $95 \%$ CL upper limit of 3 monopoles pair events produced. The upper limits on the cross sections for model A and $\mathrm{B}$, and for monopoles of different charges are shown in Fig. 64.

The direct search for magnetic monopoles has been performed with different techniques in various fields of physics. A number of papers on magnetic monopole searches have been published, in cosmic rays [297-302], in matter [303306] and at colliders [307-314]. At colliders, although a universal production mechanism can be postulated, the comparison of the cross section upper limit obtained in the H1 analysis with those of other colliders in different types of collisions $\left(e^{+} e^{-}, p \bar{p}, p p\right)$ is difficult. Recently, the ATLAS Collaboration has published [315] a search for magnetic monopoles and stable particles with high electric charge, setting a model-independent upper limit on the production cross section of $0.5 \mathrm{fb}$ for signal particles with magnetic charge $0.5 g_{D} \leq|g| \leq 2.0 g_{D}$ and electric charge in the range between 20 and 60 times the elementary charge, with masses between 200 and $2500 \mathrm{GeV}$. This result is valid in well-defined fiducial regions of high and uniform event selection efficiency. The $\mathrm{H} 1$ result described above remains the only one obtained in $e^{+} p$ collisions at high energy.

\section{Summary and outlook}

The HERA ep collider at DESY is a unique machine, which has brought a unrivalled insight into the structure of the pro- 


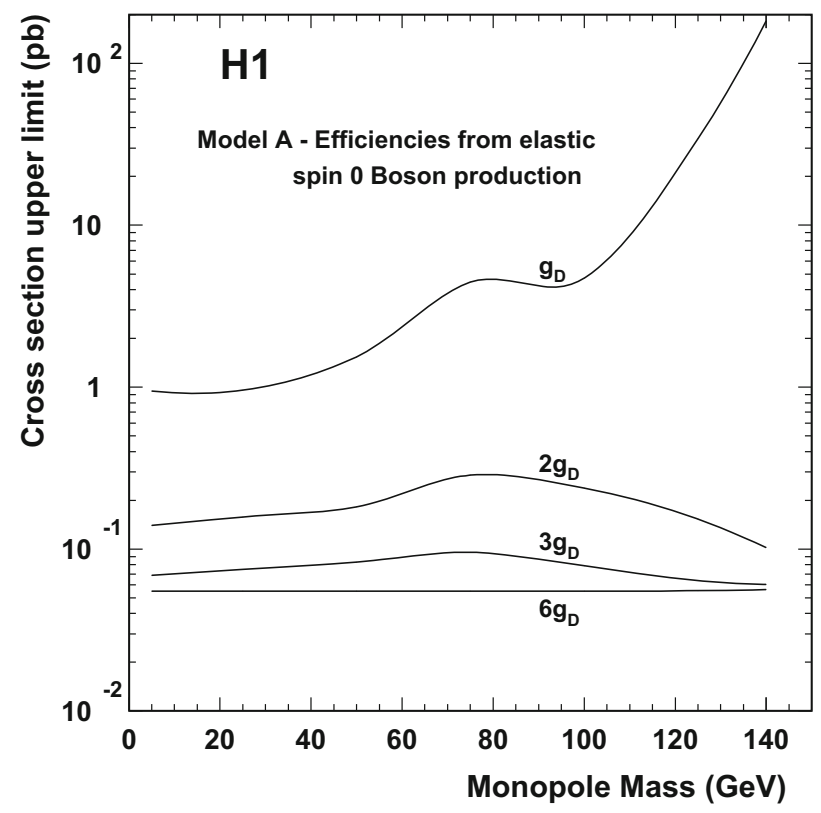

Fig. 64 Upper limits on the cross section for the production of a monopole-antimonopole pair, determined within the context of model A (left) and model B (right), for monopole-antimonopole pair produc-

ton via the precision analysis of deep inelastic scattering over many orders of magnitude in Bjorken $x$ and negative four-momentum transfer squared $Q^{2}$. The data harvest collected by the $\mathrm{H} 1$ and ZEUS experiments has also provided the opportunity to search for rare processes and physics beyond the Standard Model. Combining the data of both experiments, resulting in a HERA data set with an integrated luminosity of $1 \mathrm{fb}^{-1}$, has resulted in an increased sensitivity to such processes and allowed a thorough search of the high $P_{T}$ kinematic region.

Cross sections of the rare production of $W$ and $Z^{0}$ bosons are measured, as well as the production of high $P_{T}$ lepton pairs via two photon exchange. These analyses have also provided tantalising glimpses at physics beyond the Standard Model, and although no significant signal excess is observed in the complete data set, a number of interesting events remain in high $P_{T}$ regions of the $\mathrm{H} 1$ analyses of their $e^{+} p$ data. The single and pair production of tau leptons is also observed at HERA, utilising both leptonic and hadronic decays of the tau.

The initial $e p$ state at HERA provides a complementary environment to searches using the $e^{+} e^{-}$collisions at LEP and the $p \bar{p}$ collisions at the Tevatron. In particular, HERA is an ideal place to search for the single, resonant production of hypothetical particles such as leptoquarks, both within the kinematic limit given by the available centre of mass energy, and beyond using contact interaction models. Modeldependent searches, such as for excited fermions and supersymmetry are also performed, as well as a general search

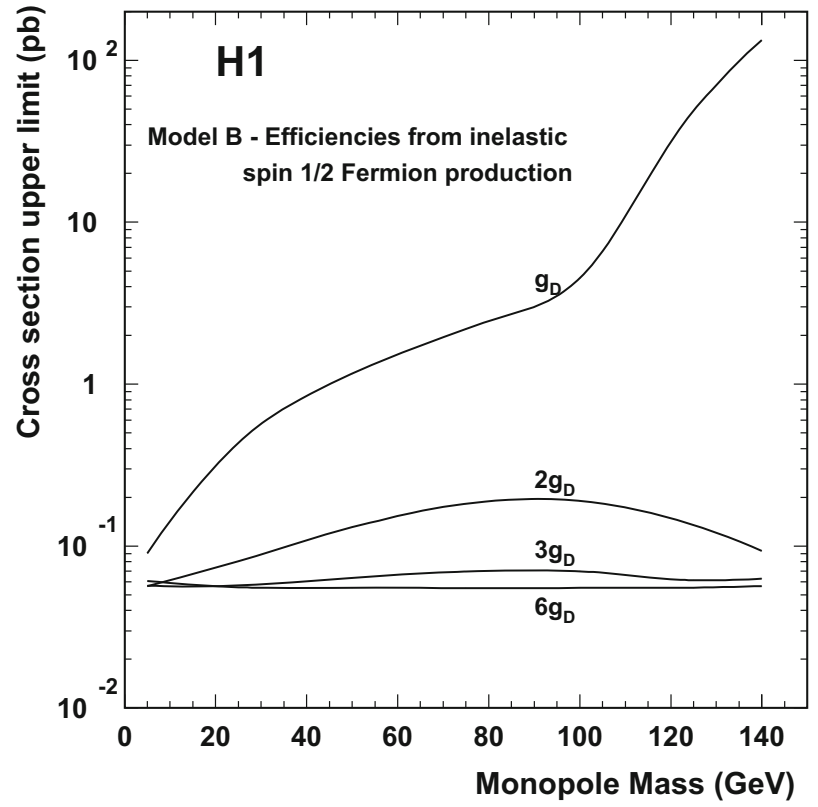

tion in $e^{+} p$ collisions as a function of monopole mass for monopoles of strength $g_{D}, 2 g_{D}, 3 g_{D}$ and $6 g_{D}$ or more

at high $P_{T}$, which confirms the results found in dedicated analyses.

No significant deviation from the Standard Model is observed in any of the search analyses performed by $\mathrm{H} 1$ and ZEUS and, where appropriate, mass-dependent model exclusion limits are derived. Whereas the limits from HERA are competitive and complementary to the analyses from colliders of the same generation, the advent of the LHC has meant that many have been superseded at the time of writing. Nevertheless, a few exceptions remain, in particular the $\mathrm{H} 1$ limits on excited neutrino production using the $e^{-} p$ data remain the best currently available, and the pair production leptoquark limits from the LHC are so far only applicable to scalar models, whereas vector leptoquark limits are set by both $\mathrm{H} 1$ and ZEUS.

Future searches in $e p$ physics may be possible if the LHeC collider is to be built, where the program will closely follow the analyses described in this review, albeit at the $\mathrm{TeV}$ energy scale. At this energy, the addition of the Higgs sector opens up a whole new physics program to explore. For example, the study of the $W W \rightarrow H \rightarrow b \bar{b}$ process, which is extremely difficult at the LHC but essentially clean at the $\mathrm{LHeC}$, allows the $W W H$ coupling to be probed and the properties of the still relatively new Higgs boson to be compared to those predicted by the Standard Model.

Open Access This article is distributed under the terms of the Creative Commons Attribution 4.0 International License (http://creativecomm ons.org/licenses/by/4.0/), which permits unrestricted use, distribution, 
and reproduction in any medium, provided you give appropriate credit to the original author(s) and the source, provide a link to the Creative Commons license, and indicate if changes were made. Funded by SCOAP ${ }^{3}$.

\section{References}

1. S.L. Glashow, Partial symmetries of weak interactions. Nucl. Phys. 22, 579 (1961)

2. S. Weinberg, A model of leptons. Phys. Rev. Lett. 19, 1264 (1967)

3. A. Salam, Weak and electromagnetic interactions. In Elementary Particle Theory, ed. by N. Svartholm (Almquist \& Wiksell), p. 367

4. G. Aad et al., Observation of a new particle in the search for the Standard Model Higgs boson with the ATLAS detector at the LHC. Phys. Lett. B 716, 1 (2012)

5. S. Chatrchyan et al., Observation of a new boson at a mass of 125 $\mathrm{GeV}$ with the CMS experiment at the LHC. Phys. Lett. B 716, 30 (2012)

6. F. Englert, R. Brout, Broken symmetry and the mass of gauge vector mesons. Phys. Rev. Lett. 13, 321 (1964)

7. P.W. Higgs, Broken symmetries and the masses of gauge bosons. Phys. Rev. Lett. 13, 508 (1964)

8. G.S. Guralnik, C.R. Hagen, T.W.B. Kibble, Global conservation laws and massless particles. Phys. Rev. Lett. 13, 585 (1964)

9. J.C. Collins, D.E. Soper, G.F. Sterman, Factorization of hard processes in QCD. Adv. Ser. Direct. High Energy Phys. 5, 1 (1988)

10. V.N. Gribov, L.N. Lipatov, Deep inelastic ep scattering in perturbation theory. Sov. J. Nucl. Phys. 15, 438 (1972)

11. G. Altarelli, G. Parisi, Asymptotic freedom in parton language. Nucl. Phys. B 126, 298 (1977)

12. Y.L. Dokshitzer, Calculation of the structure functions for deep inelastic scattering and $e^{+} e^{-}$annihilation by perturbation theory in Quantum Chromodynamics. Sov. Phys. JETP 46, 641 (1977)

13. HERA: a proposal for a large electron proton beam facility at DESY. DESY-HERA-81-10 (1981)

14. J.L. Abelleira Fernandez et al., A large hadron electron collider at CERN: report on the physics and design concepts for machine and detector. J. Phys. G 39, 075001 (2012)

15. I. Abt et al., The H1 detector at HERA. Nucl. Instrum. Methods A 386, 310 (1997)

16. I. Abt et al., The tracking, calorimeter and muon detectors of the H1 experiment at HERA. Nucl. Instrum. Methods A 386, 348 (1997)

17. ZEUS Coll. U. Holm (ed.), The ZEUS Detector. Status report (unpublished). DESY (1993). http://www-zeus.desy.de/ bluebook/bluebook.html

18. K. Ackerstaff et al., The HERMES spectrometer. Nucl. Instrum. Methods A 417, 230 (1998)

19. E.P. Hartouni, M. Kreisler, G. Van Apeldoorn, H. van der Graaf, W. Ruckstuhl, et al., HERA-B: an experiment to study CP violation in the B system using an internal target at the HERA proton ring. Design report, DESY-PRC-95-01 (1995)

20. HERA-B: a report on status and prospects October 2000. Executive summary. DESY-PRC-00-04 (2000)

21. HERA luminosity upgrade report for funding agencies. DESYPRC-98-03 (1998)

22. L. Suszycki, Luminosity monitoring, photon tagging and QED tests, in Proceedings of the HERA Workshop 1987, ed. by R.D. Peccei (DESY, Hamburg, 1988), p. 505

23. S. Levonian, Luminosity measurements with $\mathrm{H} 1$ luminosity monitor, in Proceedings of the Harz Seminar, ed. by F. Willeke (Bad Lauterberg, 1992, DESY-HERA-92-07), p. 247
24. T. Ahmed et al., Experimental study of hard photon radiation processes at HERA. Z. Phys. C 66, 529 (1995)

25. J. Andruszków et al., First measurement of HERA luminosity by ZEUS lumi monitor. DESY-92-066 (1993)

26. M. Derrick et al., Measurement of total and partial photon proton cross-sections at $180 \mathrm{GeV}$ center-of-mass energy. Z. Phys. C 63, 391 (1994)

27. J. Andruszków et al., Luminosity measurement in the ZEUS experiment. Acta Phys. Pol. B 32, 2025 (1994)

28. M. Helbich et al., The spectrometer system for measuring ZEUS luminosity at HERA. Nucl. Instrum. Methods A 565, 572 (2006)

29. F.D. Aaron et al., Determination of the integrated luminosity at HERA using elastic QED Compton events. Eur. Phys. J. C 72, 2163 (2012) (erratum. Eur. Phys. J. C 74, 2733 (2012)

30. A.A. Sokolov, I.M. Ternov, On polarization and spin effects in the theory of synchrotron radiation. Sov. Phys. Dokl. 8, 1203 (1964)

31. V.N. Baier, V.A. Khoze, Determination of the transverse polarization of high-energy electrons. Sov. J. Nucl. Phys. B 9, 238 (1969)

32. D.P. Barber et al., The HERA polarimeter and the first observation of electron spin polarization at HERA. Nucl. Instrum. Methods A 329, 79 (1993)

33. M. Beckmann et al., The longitudinal polarimeter at HERA. Nucl. Instrum. Methods A 479, 334 (2002)

34. B. Sobloher, R. Fabbri, T. Behnke, J. Olsson, D. Pitzl, et al., Polarisation at HERA: reanalysis of the HERA II polarimeter data. DESY-11-259 (2012)

35. C. Lippmann, Particle identification. Nucl. Instrum. Methods A 666, 148 (2012)

36. R. Kogler, Measurement of jet production in deep-inelastic ep scattering at HERA (2011)

37. D. Pitzl, O. Behnke, M. Biddulph, K. Bosiger, R. Eichler et al., The H1 silicon vertex detector. Nucl. Instrum. Methods A 454, 334 (2000)

38. W. Eick, H. Henschel, H.H. Kaufmann, M. Klein, P. Kostka et al., Development of the H1 backward silicon strip detector. Nucl. Instrum. Methods A 386, 81 (1997)

39. B. Andrieu et al., The H1 liquid argon calorimeter system. Nucl. Instrum. Methods A 336, 460 (1993)

40. H. Wellisch et al., Hadronic calibration of the H1 LAr calorimeter using software weighting techniques. H1 Internal Note, H1-IN346 (1994)

41. B. Andrieu et al., Results from pion calibration runs for the H1 liquid argon calorimeter and comparisons with simulations. Nucl. Instrum. Methods A 336, 499 (1993)

42. B. Andrieu et al., Beam tests and calibration of the H1 liquid argon calorimeter with electrons. Nucl. Instrum. Methods A 350, $57(1994)$

43. R.D. Appuhn et al., The H1 lead / scintillating fiber calorimeter. Nucl. Instrum. Methods A 386, 397 (1997)

44. T. Nicholls et al., Performance of an electromagnetic lead/scintillating fiber calorimeter for the H1 detector. Nucl. Instrum. Methods A 374, 149 (1996)

45. R.D. Appuhn et al., H1 backward upgrade with a SPACAL calorimeter: the hadronic section. DESY-96-013 (1996)

46. N. Harnew et al., Vertex triggering using time difference measurements in the ZEUS central tracking detector. Nucl. Instrum. Methods A 279, 290 (1989)

47. B. Foster et al., The performance of the ZEUS central tracking detector z-by-timing electronics in a transputer based data acquisition system. Nucl. Phys. Proc. Suppl. B 32, 181 (1993)

48. B. Foster et al., The design and construction of the ZEUS central tracking detector. Nucl. Instrum. Methods A 338, 254 (1994)

49. A. Polini et al., The design and performance of the ZEUS micro vertex detector. Nucl. Instrum. Methods A 581, 656 (2007)

50. S. Fourletov, Straw tube tracking detector (STT) for ZEUS. Nucl. Instrum. Methods A 535, 191 (2004) 
51. M. Derrick et al., Design and construction of the ZEUS barrel calorimeter. Nucl. Instrum. Methods A 309, 77 (1991)

52. A. Andresen et al., Construction and beam test of the ZEUS forward and rear calorimeter. Nucl. Instrum. Methods A 309, 101 (1991)

53. A. Caldwell et al., Design and implementation of a high precision readout system for the ZEUS calorimeter. Nucl. Instrum. Methods A 321, 356 (1992)

54. A. Bernstein et al., Beam tests of the ZEUS barrel calorimeter. Nucl. Instrum. Methods A 336, 23 (1993)

55. A. Bamberger et al., The presampler for the forward and rear calorimeter in the ZEUS detector. Nucl. Instrum. Methods A 382, 419 (1996)

56. S. Magill, S. Chekanov, Jet energy corrections with the ZEUS barrel preshower detector, in Proceedings of the IX International Conference on Calorimetry, ed. by B. Aubert (Frascati Physics Series, Annecy, 2000), vol. XXI, p. 625

57. A. Dwurazny et al., Experimental study of electron-hadron separation in calorimeters using silicon diodes. Nucl. Instrum. Methods A 277, 176 (1989)

58. G. Abbiendi et al., The ZEUS barrel and rear muon detector. Nucl. Instrum. Methods A 333, 342 (1993)

59. H. Abramowicz et al., Intercalibration of the ZEUS high resolution and backing calorimeters. Nucl. Instrum. Methods A 313, 126 (1992)

60. F.D. Aaron et al., Inclusive deep inelastic scattering at high $Q^{2}$ with longitudinally polarised lepton beams at HERA. JHEP $\mathbf{1 2 0 9}$, 061 (2012)

61. G.P. Salam, Towards jetography. Eur. Phys. J. C67, 637 (2010)

62. S. Catani, Y.L. Dokshitzer, M.H. Seymour, B.R. Webber, Longitudinally invariant $k_{t}$ clustering algorithms for hadron-hadron collisions. Nucl. Phys. B 406, 187 (1993)

63. V. Andreev et al., Measurement of multijet production in ep collisions at high $Q^{2}$ and determination of the strong coupling $\alpha_{s}$. Eur. Phys. J. C 75(2), 65 (2015)

64. M. Cacciari, G.P. Salam, G. Soyez, The anti-k(t) jet clustering algorithm. JHEP 0804, 063 (2008)

65. M. Derrick et al., Measurement of the $F_{2}$ structure function in deep inelastic $e^{+} p$ scattering using 1994 data from the ZEUS detector at HERA. Z. Phys. C 72, 399 (1996)

66. F. Jacquet, A. Blondel, Detection of the charged current eventmethod II, in Proceedings of the Study of an ep Facility for Europe, ed. by U. Amaldi (Hamburg, 1979, DESY 79/48), p. 391

67. C.G. Callan Jr., D.J. Gross, High-energy electroproduction and the constitution of the electric current. Phys. Rev. Lett. 22, 156 (1969)

68. U. Bassler, G. Bernardi, On the kinematic reconstruction of deep inelastic scattering at HERA: the sigma method. Nucl. Instrum. Methods A 361, 197 (1995)

69. U. Bassler, G. Bernardi, Structure function measurements and kinematic reconstruction at HERA. Nucl. Instrum. Methods A 426, 583 (1999)

70. B. Heinemann, Measurement of charged current and neutral current cross-sections in positron proton collisions at $\sqrt{s} \simeq 300 \mathrm{GeV}$ (1999)

71. S. Bentvelsen, J. Engelen, P. Kooijman. Reconstruction of $\left(x, Q^{2}\right)$ and extration of structure functions in neutral current scattering, in Proceedings of the Workshop "Physics at HERA", ed. by W. Buchmüller, G. Ingelman (DESY, Hamburg, 1991), vol. 1, p. 23

72. K. Hoeger, Measurement of $x, y, Q^{2}$ in neutral current events, in Proceedings of the Workshop "Physics at HERA", ed. by W. Buchmüller, G. Ingelman (DESY, Hamburg, 1991), vol. 1, p. 43

73. J.C. Collins, D.E. Soper, G.F. Sterman, Soft gluons and factorization. Nucl. Phys. B 308, 833 (1988)

74. I.G. Corcella, G. Knowles, G. Marchesini, S. Moretti, K. Odagiri et al., HERWIG 6: an event generator for hadron emission reactions with interfering gluons (including supersymmetric processes). JHEP 01, 010 (2001)

75. B. Andersson, G. Gustafson, G. Ingelman, T. Sjöstrand, Parton fragmentation and string dynamics. Phys. Rep. 97, 31 (1983)

76. T. Sjöstrand, S. Mrenna, P.Z. Skands, PYTHIA 6.4 physics and manual. JHEP 05, 026 (2006)

77. T. Sjöstrand, M. Bengtsson, The Lund Monte Carlo for jet fragmentation and $e^{+} e^{-}$physics. Jetset version 6.3: an update. Comput. Phys. Commun. 43, 367 (1987)

78. R. Brun, GEANT 3.13. CERN DD/EE 84-1 (1984)

79. V.N. Gribov, L.N. Lipatov, $e^{+} e^{-}$pair annihilation and deep inelastic ep scattering in perturbation theory. Sov. J. Nucl. Phys. 15, 675 (1972)

80. L.N. Lipatov, The parton model and perturbation theory. Sov. J. Nucl. Phys. 20, 94 (1975)

81. H. Abramowicz et al., Combination of measurements of inclusive deep inelastic $e^{ \pm} p$ scattering cross sections and QCD analysis of HERA data. Eur. Phys. J. C 75(12), 580 (2015)

82. F.D. Aaron et al., Measurement of the inclusive $e p$ scattering cross section at low $Q^{2}$ and $x$ at HERA. Eur. Phys. J. C 63, 625 (2009)

83. A. Glazov, Averaging of DIS cross section data, in Proceedings of the 13th International Workshop on Deep Inelastic Scattering (DIS 2005), ed. by W.H. Smith, S.R. Dasu (Madison, Wisconsin, 2005), vol. 792, p. 237

84. J. Breitweg et al., Measurement of the proton structure function $F_{2}$ at very low $Q^{2}$ at HERA. Phys. Lett. B 487, 53 (2000)

85. J. Breitweg et al., ZEUS results on the measurement and phenomenology of $F_{2}$ at low $x$ and low $Q^{2}$. Eur. Phys. J. C 7, 609 (1999)

86. C. Adloff et al., Measurement of inclusive jet cross-sections in photoproduction at HERA. Nucl. Phys. B 497, 3 (1997)

87. G.A. Schuler, H. Spiesberger, DJANGO: the interface for the event generators HERACLES and LEPTO, in Proceedings of the Workshop "Physics at HERA", ed. by W. Buchmüller and G. Ingelman (DESY, Hamburg, 1991), vol. 3, p. 1419

88. A. Kwiatkowski, H. Spiesberger, H.-J. Möhring, Heracles: an event generator for $e p$ interactions at HERA energies including radiative processes: version 1.0. Comput. Phys. Commun. 69, 155 (1992)

89. G. Ingelman. LEPTO version 6.1: the Lund Monte Carlo for deep inelastic lepton-nucleon scattering, in Procedings of the Workshop "Physics at HERA", ed. by W. Buchmüller and G. Ingelman (DESY, Hamburg, 1991), vol. 3, p. 1366

90. L. Lönnblad, ARIADNE version 4: a program for simulation of QCD cascades implementing the color dipole model. Comput. Phys. Commun. 71, 15 (1992)

91. A.C. Benvenuti et al., A high statistics measurement of the proton structure functions $F_{2}\left(x, Q^{2}\right)$ and $r$ from deep inelastic muon scattering at high $Q^{2}$. Phys. Lett. B 223, 485 (1989)

92. M. Arneodo et al., Measurement of the proton and deuteron structure functions, $F_{2}^{p}$ and $F_{2}^{d}$, and of the ratio sigma-L / sigma-T. Nucl. Phys. B 483, 3 (1997)

93. G. Altarelli, G. Martinelli, Transverse momentum of jets in electroproduction from quantum chromodynamics. Phys. Lett. B 76, 89 (1978)

94. J.J. Aubert et al., Measurement of $r=\sigma_{l} / \sigma_{t}$ in deep inelastic muon-proton scattering. Phys. Lett. B 121, 87 (1983)

95. L.W. Whitlow, S. Rock, A. Bodek, E.M. Riordan, S. Dasu, A precise extraction of $\mathrm{R}=$ sigma- $\mathrm{L} /$ sigma-T from a global analysis of the SLAC deep inelastic $e p$ and $e d$ scattering cross-sections. Phys. Lett. B 250, 193 (1990)

96. V. Andreev et al., Measurement of inclusive $e p$ cross sections at high $Q^{2}$ at $\sqrt{s}=225$ and $252 \mathrm{GeV}$ and of the longitudinal proton structure function $F_{L}$ at HERA. Eur. Phys. J. C 74(4), 2814 (2014)

97. S. Chekanov et al., Measurement of the longitudinal proton structure function at HERA. Phys. Lett. B 682, 8 (2009) 
98. T. Ahmed et al., First measurement of the charged current crosssection at HERA. Phys. Lett. B 324, 241 (1994)

99. M. Derrick et al., Measurement of charged and neutral current $e^{-} p$ deep inelastic scattering cross-sections at high $Q^{2}$. Phys. Rev. Lett. 75, 1006 (1995)

100. T. Abe, GRAPE dilepton (version 1.1): a generator for dilepton production in ep collisions. Comput. Phys. Commun. 136, 126 (2001)

101. U. Baur, J.A.M. Vermaseren, D. Zeppenfeld, Electroweak vector boson production in high-energy ep collisions. Nucl. Phys. B 375, 3 (1992)

102. C. Adloff et al., Measurement of neutral and charged current crosssections in positron proton collisions at large momentum transfer. Eur. Phys. J. C 13, 609 (2000)

103. F.D. Aaron et al., Combined measurement and QCD analysis of the inclusive $e^{ \pm} p$ scattering cross sections at HERA. JHEP 01, 109 (2010)

104. S. Fanchiotti, B.A. Kniehl, A. Sirlin, Incorporation of QCD effects in basic corrections of the electroweak theory. Phys. Rev. D 48, 307 (1993)

105. W.T. Giele, S. Keller, Implications of hadron collider observables on parton distribution function uncertainties. Phys. Rev. D 58, 094023 (1998)

106. H. Abramowicz et al., Combination and QCD analysis of charm production cross section measurements in deep-inelastic ep scattering at HERA. Eur. Phys. J. C 73(2), 2311 (2013)

107. R. Devenish, A. Cooper-Sarkar, Deep inelastic scattering (Oxford University Press, Oxford, 2003)

108. V. Radescu, Combination and QCD analysis of the HERA inclusive cross sections, in Proceedings of the 35th International Conference on High Energy Physics ( PoS, ICHEP, 2010), p. 168

109. A.M. Cooper-Sarkar, PDF fits at HERA, in Proceedings of the 21 st International Europhysics Conference on High Energy Physics (PoS, EPS-HEP, 2011), p. 320

110. H. Abramowicz et al., Measurement of high- $Q^{2}$ charged current deep inelastic scattering cross sections with a longitudinally polarised positron beam at HERA. Eur. Phys. J. C 70, 945 (2010)

111. E. Eichten, K.D. Lane, M.E. Peskin, New tests for quark and lepton substructure. Phys. Rev. Lett. 50, 811 (1983)

112. R. Ruckl, Effects of compositeness in deep inelastic scattering. Phys. Lett. B 129, 363 (1983)

113. R. Ruckl, Probing lepton and quark substructure in polarized $e^{\mp} N$ scattering. Nucl. Phys. B 234, 91 (1984)

114. P. Haberl, F. Schrempp, H.U. Martyn, Contact interactions and new heavy bosons at HERA - a model independent analysis, in Proceedings of the Workshop "Physics at HERA", ed. by W. Buchmüller and G. Ingelman (DESY, Hamburg, 1991), vol. 2, p. 1133

115. W. Buchmüller, R. Ruckl, D. Wyler, Leptoquarks in lepton-quark collisions. Phys. Lett. B 191, 442 (1987) (erratum. Phys. Lett. B 448, 320 (1999)

116. F. Schrempp, Leptoquarks and leptogluons - theoretical perspectives, in Proceedings of the Workshop "Physics at HERA", ed. by W. Buchmüller and G. Ingelman (DESY, Hamburg, 1991), vol. 2, p. 1034

117. N. Arkani-Hamed, S. Dimopoulos, G.R. Dvali, The hierarchy problem and new dimensions at a millimeter. Phys. Lett. B 429, 263 (1998)

118. I. Antoniadis, N. Arkani-Hamed, S. Dimopoulos, G.R. Dvali, New dimensions at a millimeter to a Fermi and superstrings at a TeV. Phys. Lett. B 436, 257 (1998)

119. N. Arkani-Hamed, S. Dimopoulos, G.R. Dvali, Phenomenology, astrophysics and cosmology of theories with submillimeter dimensions and TeV scale quantum gravity. Phys. Rev. D 59, 086004 (1999)
120. S. Chekanov et al., Search for contact interactions, large extra dimensions and finite quark radius in $e p$ collisions at HERA. Phys. Lett. B 591, 23 (2004)

121. C. Adloff et al., Search for compositeness, leptoquarks and large extra dimensions in $e q$ contact interactions at HERA. Phys. Lett. B 479, 358 (2000)

122. G.F. Giudice, R. Rattazzi, J.D. Wells, Quantum gravity and extra dimensions at high-energy colliders. Nucl. Phys. B 544, 3 (1999)

123. K. Cheung, Global lepton quark neutral current constraint on low scale gravity model. Phys. Lett. B 460, 383 (1999)

124. J. Breitweg et al., Search for contact interactions in deep inelastic $e^{+} p \rightarrow e^{+} X$ scattering at HERA. Eur. Phys. J. C 14, 239 (2000)

125. C. Adloff et al., Search for new physics in $e^{ \pm} q$ contact interactions at HERA. Phys. Lett. B 568, 35 (2003)

126. F.D. Aaron et al., Search for contact interactions in $e^{ \pm} p$ collisions at HERA. Phys. Lett. B 705, 52 (2011)

127. J. Pumplin, D.R. Stump, J. Huston, H.L. Lai, P.M. Nadolsky, W.K. Tung, New generation of parton distributions with uncertainties from global QCD analysis. JHEP 07, 012 (2002)

128. G. Aad et al., Search for contact interactions and large extra dimensions in the dilepton channel using proton-proton collisions at $\sqrt{s}$ $=8 \mathrm{TeV}$ with the ATLAS detector. Eur. Phys. J. C 74(12), 3134 (2014)

129. J.C. Pati, A. Salam, Lepton number as the fourth color. Phys. Rev. D 10, 275 (1974) (erratum. Phys. Rev. D 11, 703 (1975)

130. H. Georgi, S.L. Glashow, Unity of all elementary particle forces. Phys. Rev. Lett. 32, 438 (1974)

131. P. Langacker, Grand unified theories and proton decay. Phys. Rep. 72, 185 (1981)

132. B. Schrempp, F. Schrempp, Light leptoquarks. Phys. Lett. B 153, 101 (1985)

133. J. Wudka, Composite leptoquarks. Phys. Lett. B 167, 337 (1986)

134. S. Dimopoulos, L. Susskind, Mass without scalars. Nucl. Phys. B 155, 237 (1979)

135. S. Dimopoulos, Technicolored signatures. Nucl. Phys. B 168, 69 (1980)

136. E. Farhi, L. Susskind, A technicolored G.U.T. Phys. Rev. D 20 , 3404 (1979)

137. E. Farhi, L. Susskind, Technicolor. Phys. Rep. 74, 277 (1981)

138. H.-P. Nilles, Supersymmetry, supergravity and particle physics. Phys. Rep. 110, 1 (1984)

139. H.E. Haber, G.L. Kane, The search for supersymmetry: probing physics beyond the Standard Model. Phys. Rep. 117, 75 (1985)

140. A. Aktas et al., Search for lepton flavour violation in ep collisions at HERA. Eur. Phys. J. C 52, 833 (2007)

141. C. Adloff et al., A search for leptoquark bosons and lepton flavor violation in $e^{+} p$ collisions at HERA. Eur. Phys. J. C 11, 447 (1999) (erratum. Eur. Phys. J. C 14, 553 (2000)

142. A. Aktas et al., Search for leptoquark bosons in $e p$ collisions at HERA. Phys. Lett. B 629, 9 (2005)

143. J. Breitweg et al., Search for resonances decaying to $e^{+}$-jet in $e^{+} p$ interactions at HERA. Eur. Phys. J. C 16, 253 (2000)

144. J. Breitweg et al., Search for resonance decays to an anti-neutrino plus jet in $e^{+} p$ scattering at DESY HERA. Phys. Rev. D 63, 052002 (2001)

145. S. Chekanov et al., A search for resonance decays to lepton + jet at HERA and limits on leptoquarks. Phys. Rev. D 68, 052004 (2003)

146. C. Adloff et al., Observation of events at very high $Q^{2}$ in $e p$ collisions at HERA. Z. Phys. C 74, 191 (1997)

147. J. Breitweg et al., Comparison of ZEUS data with standard model predictions for $e^{+} p \rightarrow e^{+} X$ scattering at high x and $Q^{2}$. Z. Phys. C 74, 207 (1997)

148. F.D. Aaron et al., Search for first generation leptoquarks in $e p$ collisions at HERA. Phys. Lett. B 704, 388 (2011) 
149. H. Abramowicz et al., Search for first-generation leptoquarks at HERA. Phys. Rev. D 86, 012005 (2012)

150. H.L. Lai, J. Huston, S. Kuhlmann, J. Morfin, F.I. Olness, J.F. Owens, J. Pumplin, W.K. Tung, Global QCD analysis of parton structure of the nucleon: CTEQ5 parton distributions. Eur. Phys. J. C 12, 375 (2000)

151. P. Bock, Computation of confidence levels for exclusion or discovery of a signal with the method of fractional event counting. JHEP 01, 080 (2007)

152. G. Abbiendi et al., Tests of the Standard Model and constraints on new physics from measurements of fermion pair production at $183 \mathrm{GeV}$ at LEP. Eur. Phys. J. C 6, 1 (1999)

153. M. Acciarri et al., Search for manifestations of new physics in fermion pair production at LEP. Phys. Lett. B 489, 81 (2000)

154. V.M. Abazov et al., Search for pair production of first-generation leptoquarks in $p \bar{p}$ collisions at $\sqrt{s}=1.96 \mathrm{TeV}$. Phys. Lett. B 681, 224 (2009)

155. V.M. Abazov et al., Search for first generation leptoquark pair production in the electron + missing energy + jets final state. Phys. Rev. D 84, 071104 (2011)

156. G. Aad et al., Search for first generation scalar leptoquarks in $p p$ collisions at $\sqrt{s}=7 \mathrm{TeV}$ with the ATLAS detector. Phys. Lett., B709:158, 2012. [Erratum. Phys. Lett. B 711, 442 (2012)

157. S. Chatrchyan et al., Search for pair production of first- and second-generation scalar leptoquarks in $p p$ collisions at $\sqrt{s}=7$ TeV. Phys. Rev. D 86, 052013 (2012)

158. G. Aad et al., Searches for scalar leptoquarks in $p p$ collisions at $\sqrt{s}=8 \mathrm{TeV}$ with the ATLAS detector. Eur. Phys. J. C 76(1), 5 (2016)

159. R. Barbieri, G. Isidori, J. Jones-Perez, P. Lodone, D.M. Straub, $U(2)$ and minimal flavour violation in supersymmetry. Eur. Phys. J. C 71, 1725 (2011)

160. S. Chekanov et al., Search for lepton-flavor violation at HERA. Eur. Phys. J. C 44, 463 (2005)

161. F.D. Aaron et al., Search for lepton flavour violation at HERA. Phys. Lett. B 701, 20 (2011)

162. A. Aktas et al., Tau lepton production in ep collisions at HERA. Eur. Phys. J. C 48, 699 (2006)

163. H. Jung, Hard diffractive scattering in high-energy ep collisions and the Monte Carlo generator RAPGAP. Comput. Phys. Commun. 86, 147 (1995)

164. R. Barate et al., Search for the Standard Model Higgs boson at LEP. Phys. Lett. B 565, 61 (2003)

165. V. Khachatryan et al., Search for third-generation scalar leptoquarks in the $t \tau$ channel in proton-proton collisions at $\sqrt{s}=8$ TeV. JHEP 07, 042 (2015)

166. V. Khachatryan et al., Search for pair production of thirdgeneration scalar leptoquarks and top squarks in proton-proton collisions at $\sqrt{(} s)=8 \mathrm{TeV}$. Phys. Lett. B 739, 229 (2014)

167. J.A.M. Vermaseren, Two photon processes at very high-energies. Nucl. Phys. B 229, 347 (1983)

168. A. Aktas et al., Multi-electron production at high transverse momenta in $e p$ collisions at HERA. Eur. Phys. J. C 31, 17 (2003)

169. A. Aktas et al., Muon pair production in ep collisions at HERA. Phys. Lett. B 583, 28 (2004)

170. F.D. Aaron et al., Multi-lepton production at high transverse momenta in ep collisions at HERA. Phys. Lett. B 668, 268 (2008)

171. S. Chekanov et al., Multi-lepton production at high transverse momentum at HERA. Phys. Lett. B 680, 13 (2009)

172. C. Berger, P. Kandel, A new generator for wide angle bremsstrahlung, in Proceedings of the Workshop "Monte Carlo Generators for HERA Physics", ed. by A.T. Doyle et al. (DESY, Hamburg, 1999), p. 596

173. F.D. Aaron et al., Multi-leptons with high transverse momentum at HERA. JHEP 10, 013 (2009)
174. A. Levy et al., Study of tau-pair production at HERA. JHEP 02, 117 (2011)

175. T. Carli, B. Koblitz, A multivariate discrimination technique based on range searching. Nucl. Instrum. Methods A 501, 576 (2003)

176. E. Accomando, S. Petrarca, Searching a doubly charged Higgs boson at HERA. Phys. Lett. B 323, 212 (1994)

177. A. Aktas et al., Search for doubly-charged Higgs boson production at HERA. Phys. Lett. B 638, 432 (2006)

178. T. Junk, Confidence level computation for combining searches with small statistics. Nucl. Instrum. Methods A 434, 435 (1999)

179. G. Abbiendi et al., Search for the single production of doubly charged Higgs bosons and constraints on their couplings from Bhabha scattering. Phys. Lett. B 577, 93 (2003)

180. G. Abbiendi et al., Search for doubly charged Higgs bosons with the OPAL detector at LEP. Phys. Lett. B 526, 221 (2002)

181. J. Abdallah et al., Search for doubly charged Higgs bosons at LEP-2. Phys. Lett. B 552, 127 (2003)

182. P. Achard et al., Search for doubly charged Higgs bosons at LEP. Phys. Lett. B 576, 18 (2003)

183. T. Aaltonen et al., Search for new physics in high $p_{T}$ like-sign dilepton events at CDF II. Phys. Rev. Lett. 107, 181801 (2011)

184. G. Aad et al., Search for new phenomena in events with three or more charged leptons in $p p$ collisions at $\sqrt{s}=8 \mathrm{TeV}$ with the ATLAS detector. JHEP 08, 138 (2015)

185. G. Aad et al., Search for anomalous production of prompt samesign lepton pairs and pair-produced doubly charged Higgs bosons with $\sqrt{s}=8 \mathrm{TeV} p p$ collisions using the ATLAS detector. JHEP 03, 041 (2015)

186. T. Ahmed et al., Observation of an $e^{+} p \rightarrow \mu+X$ event with high transverse momenta at HERA. DESY-94-248 (1994)

187. K.-P.O. Diener, C. Schwanenberger, M. Spira, Photoproduction of $W$ bosons at HERA: reweighting method for implementing QCD corrections in Monte Carlo programs (2003)

188. K.-P.O. Diener, C. Schwanenberger, M. Spira, Photoproduction of $W$ bosons at HERA: QCD corrections. Eur. Phys. J. C 25, 405 (2002)

189. P. Nason, R. Ruckl, M. Spira, A note on $W$ production at HERA. J. Phys. G 25, 1434 (1999)

190. M. Spira, $W$ boson production at NLO, in Proceedings of the Workshop "Monte Carlo Generators for HERA Physics", ed. by A.T. Doyle et al. (DESY, Hamburg, 1999), p. 623

191. C. Adloff et al., Observation of events with an isolated high-energy lepton and missing transverse momentum at HERA. Eur. Phys. J. C 5, 575 (1998)

192. V. Andreev et al., Isolated electrons and muons in events with missing transverse momentum at HERA. Phys. Lett. B 561, 241 (2003)

193. J. Breitweg et al., $W$ production and the search for events with an isolated high-energy lepton and missing transverse momentum at HERA. Phys. Lett. B 471, 411 (2000)

194. S. Chekanov et al., Search for single top production in ep collisions at HERA. Phys. Lett. B 559, 153 (2003) (addendum: DESY03-188, 2003)

195. F.D. Aaron et al., Events with isolated leptons and missing transverse momentum and measurement of $W$ production at HERA. Eur. Phys. J. C 64, 251 (2009)

196. S. Chekanov et al., Search for events with an isolated lepton and missing transverse momentum and a measurement of $W$ production at HERA. Phys. Lett. B 672, 106 (2009)

197. C. Diaconu, Events with isolated leptons and missing transverse momentum at HERA, in Proceedings of the 33rd International Conference on High Energy Physics (ICHEP 2006), ed. by A. Sissakian et al., C060726, p. 1151

198. F.D. Aaron et al., Events with an isolated lepton and missing transverse momentum and measurement of $W$ production at HERA. JHEP 03, 035 (2010) 
199. U. Baur, D. Zeppenfeld, Measuring the $W W \gamma$ vertex in single $W$ production at $e p$ colliders. Nucl. Phys. B 325, 253 (1989)

200. K. Hagiwara, R.D. Peccei, D. Zeppenfeld, K. Hikasa, Probing the weak boson sector in $e^{+} e^{-} \rightarrow W^{+} W^{-}$. Nucl. Phys. B 282, 253 (1987)

201. S. Schael et al., Improved measurement of the triple gauge-boson couplings $\gamma W W$ and $Z W W$ in $e^{+} e^{-}$collisions. Phys. Lett. B 614, 7 (2005)

202. J. Abdallah et al., Study of $W$ boson polarisations and triple gauge boson couplings in the reaction $e^{+} e^{-} \rightarrow W^{+} W^{-}$at LEP 2. Eur. Phys. J. C 54, 345 (2008)

203. P. Achard et al., Measurement of triple gauge boson couplings of the $W$ boson at LEP. Phys. Lett. B 586, 151 (2004)

204. G. Abbiendi et al., Measurement of charged current triple gauge boson couplings using $W$ pairs at LEP. Eur. Phys. J. C 33, 463 (2004)

205. S. Chekanov et al., Isolated tau leptons in events with large missing transverse momentum at HERA. Phys. Lett. B 583, 41 (2004)

206. M. Schott, M. Dunford, Review of single vector boson production in $p p$ collisions at $\sqrt{s}=7 \mathrm{TeV}$. Eur. Phys. J. C 74, 2916 (2014)

207. G.A. Schuler, Heavy flavor production at HERA. Nucl. Phys. B 299, 21 (1988)

208. U. Baur, J.J. van der Bij, Top quark production at HERA. Nucl. Phys. B 304, 451 (1988)

209. J.J. van der Bij, G.J. van Oldenborgh, QCD radiative corrections to charged current heavy quark production. Z. Phys. C 51, 477 (1991)

210. T. Stelzer, Z. Sullivan, S. Willenbrock, Single top quark production via $W$-gluon fusion at next-to-leading order. Phys. Rev. D 56, 5919 (1997)

211. S. Moretti, K. Odagiri, Single top production at future ep colliders. Phys. Rev. D 57, 3040 (1998)

212. D. Atwood, L. Reina, A. Soni, Probing flavor changing top-charmscalar interactions in $e^{+} e^{-}$collisions. Phys. Rev. D 53, 1199 (1996)

213. G.M. de Divitiis, R. Petronzio, L. Silvestrini, Flavor changing top decays in supersymmetric extensions of the Standard Model. Nucl. Phys. B 504, 45 (1997)

214. R.D. Peccei, X. Zhang, Dynamical symmetry breaking and universality breakdown. Nucl. Phys. B 337, 269 (1990)

215. H. Fritzsch, D. Holtmannspotter, The production of single $t$ quarks at LEP and HERA. Phys. Lett. B 457, 186 (1999)

216. E. Boos et al., CompHEP 4.5 status report, in Proceedings of the 12th International Workshop on Advanced Computing and Analysis Techniques in Physics Research (PoS, ACAT2008), p. 008

217. A. Aktas et al., Search for single top quark production in $e p$ collisions at HERA. Eur. Phys. J. C 33, 9 (2004)

218. F.D. Aaron et al., Search for single top quark production at HERA. Phys. Lett. B 678, 450 (2009)

219. H. Abramowicz et al., Search for single-top production in $e p$ collisions at HERA. Phys. Lett. B 708, 27 (2012)

220. A. Belyaev, N. Kidonakis, QCD corrections to FCNC single top production at HERA. Phys. Rev. D 65, 037501 (2002)

221. A. Heister et al., Search for single top production in $e^{+} e^{-}$collisions at $\sqrt{s}$ up to $209 \mathrm{GeV}$. Phys. Lett. B 543, 173 (2002)

222. F. Abe et al., Search for flavor-changing neutral current decays of the top quark in $p \bar{p}$ collisions at $\sqrt{s}=1.8 \mathrm{TeV}$. Phys. Rev. Lett. 80, 2525 (1998)

223. T. Aaltonen et al., Search for the flavor changing neutral current decay $t \rightarrow Z q$ in $p \bar{p}$ collisions at $\sqrt{s}=1.96 \mathrm{TeV}$. Phys. Rev. Lett. 101, 192002 (2008)

224. V.M. Abazov et al., Search for flavor changing neutral currents in decays of top quarks. Phys. Lett. B 701, 313 (2011)
225. G. Aad et al., A search for flavour changing neutral currents in top-quark decays in $p p$ collision data collected with the ATLAS detector at $\sqrt{s}=7$ TeV. JHEP 09, 139 (2012)

226. G. Aad et al., Search for FCNC single top-quark production at $\sqrt{s}=7 \mathrm{TeV}$ with the ATLAS detector. Phys. Lett. B 712, 351 (2012)

227. S. Chatrchyan et al., Search for flavor-changing neutral currents in top-quark decays $t \rightarrow Z q$ in $p p$ collisions at $\sqrt{s}=8 \mathrm{TeV}$. Phys. Rev. Lett. 112(17), 171802 (2014)

228. F.D. Aaron et al., A general search for new phenomena at HERA. Phys. Lett. B 674, 257 (2009)

229. A. Aktas et al., A general search for new phenomena in $e p$ scattering at HERA. Phys. Lett. B 602, 14 (2004)

230. S. Geer, T. Asakawa, The analysis of multi-jet events produced at high-energy hadron colliders. Phys. Rev. D 53, 4793 (1996)

231. F.D. Aaron et al., Search for excited electrons in $e p$ collisions at HERA. Phys. Lett. B 666, 131 (2008)

232. F.D. Aaron et al., A search for excited neutrinos in $e^{-} p$ collisions at HERA. Phys. Lett. B 663, 382 (2008)

233. H. Harari, Composite models for quarks and leptons. Phys. Rep. 104, 159 (1984)

234. C. Adloff et al., Search for excited electrons at HERA. Phys. Lett. B 548, 35 (2002)

235. C. Adloff et al., Search for excited neutrinos at HERA. Phys. Lett. B 525, 9 (2002)

236. C. Adloff et al., A search for excited fermions at HERA. Eur. Phys. J. C 17, 567 (2000)

237. S. Chekanov et al., Searches for excited fermions in ep collisions at HERA. Phys. Lett. B 549, 32 (2002)

238. F.D. Aaron et al., Search for excited quarks in $e p$ collisions at HERA. Phys. Lett. B 678, 335 (2009)

239. K. Hagiwara, D. Zeppenfeld, S. Komamiya, Excited lepton production at LEP and HERA. Z. Phys. C 29, 115 (1985)

240. F. Boudjema, A. Djouadi, J.L. Kneur, Excited fermions at $e^{+} e^{-}$ and $e P$ colliders. Z. Phys. C 57, 425 (1993)

241. U. Baur, M. Spira, P.M. Zerwas, Excited quark and lepton production at hadron colliders. Phys. Rev. D 42, 815 (1990)

242. S.J. Brodsky, S.D. Drell, The anomalous magnetic moment and limits on fermion substructure. Phys. Rev. D 22, 2236 (1980)

243. F.M. Renard, Limits on masses and couplings of excited electrons and muons. Phys. Lett. B 116, 264 (1982)

244. E. Boos, V. Bunichev, M. Dubinin, L. Dudko, V. Ilyin, A. Kryukov, V. Edneral, V. Savrin, A. Semenov, A. Sherstnev, CompHEP 4.4: automatic computations from Lagrangians to events. Nucl. Instrum. Methods A 534, 250 (2004)

245. A. Pukhov, E. Boos, M. Dubinin, V. Edneral, V. Ilyin, D. Kovalenko, A. Kryukov, V. Savrin, S. Shichanin, A. Semenov, CompHEP: a package for evaluation of Feynman diagrams and integration over multiparticle phase space. NP-MSU-98-41-542 (1999)

246. T. Köhler. Exotic proesses at HERA: the event generator COMPOS, in Proceedings of the Workshop "Physics at HERA", ed. by W. Buchmüller and G. Ingelman (DESY, Hamburg, 1991), vol. 3 , p. 1526

247. G. Abbiendi et al., Search for charged excited leptons in $e^{+} e^{-}$ collisions at $\sqrt{s}=183-209 \mathrm{GeV}$. Phys. Lett. B 544, 57 (2002)

248. J. Abdallah et al., Determination of the $e^{+} e^{-} \rightarrow \gamma \gamma(\gamma)$ crosssection at LEP 2. Eur. Phys. J. C 37, 405 (2004)

249. P. Achard et al., Search for excited leptons at LEP. Phys. Lett. B 568, $23(2003)$

250. P. Abreu et al., Search for composite and exotic fermions at LEP-2. Eur. Phys. J. C 8, 41 (1999)

251. D. Acosta et al., Search for excited and exotic electrons in the $e \gamma$ decay channel in $p \bar{p}$ collisions at $\sqrt{s}=1.96 \mathrm{TeV}$. Phys. Rev. Lett. 94, 101802 (2005) 
252. G. Aad et al., Search for excited electrons and muons in $\sqrt{s}=8$ $\mathrm{TeV}$ proton-proton collisions with the ATLAS detector. New J. Phys. 15, 093011 (2013)

253. G. Aad et al., Search for new phenomena in photon + jet events collected in proton-proton collisions at $\mathrm{sqrt}(\mathrm{s})=8 \mathrm{TeV}$ with the ATLAS detector. Phys. Lett. B 728, 562 (2014)

254. J. Wess, B. Zumino, Supergauge transformations in fourdimensions. Nucl. Phys. B 70, 39 (1974)

255. S. Weinberg, Supersymmetry at ordinary energies. 1. Masses and conservation laws. Phys. Rev. D 26, 287 (1982)

256. N. Sakai, T. Yanagida, Proton decay in a class of supersymmetric grand unified models. Nucl. Phys. B 197, 533 (1982)

257. J. Butterworth, H.K. Dreiner, $R$-parity violation at HERA. Nucl. Phys. B 397, 3 (1993)

258. A. Aktas et al., Search for squark production in $R$-parity violating supersymmetry at HERA. Eur. Phys. J. C 36, 425 (2004)

259. S. Chekanov et al., Search for stop production in R-parityviolating supersymmetry at HERA. Eur. Phys. J. C 50, 269 (2007)

260 . F.D. Aaron et al., Search for squarks in $R$-parity violating supersymmetry in ep collisions at HERA. Eur. Phys. J. C 71, 1572 (2011)

261. T. Plehn, H. Spiesberger, M. Spira, P.M. Zerwas, Formation and decay of scalar leptoquarks / squarks in ep collisions. Z. Phys. C 74, 611 (1997)

262. K. Rosenbauer, Suche nach Leptoquarks und Leptogluonen im H1-Experiment bei HERA (1995)

263. N. Ghodbane, SUSYGEN3: an event generator for linear colliders, in Proceedings of the 4th International Workshop on Linear Colliders (LCWS 99), LYCEN-9992, p. 745

264. C. Adloff et al., Measurement of dijet cross-sections in photoproduction at HERA. Eur. Phys. J. C 25, 13 (2002)

265. P. Langacker, Parity violation in muonic atoms and cesium. Phys. Lett. B 256, 277 (1991)

266. D.Z. Freedman, P. van Nieuwenhuizen, S. Ferrara, Progress toward a theory of supergravity. Phys. Rev. D 13, 3214 (1976)

267. M. Drees, M.M. Nojiri, Radiative symmetry breaking in minimal $N=1$ supergravity with large Yukawa couplings. Nucl. Phys. B 369, 54 (1992)

268. H. Baer, X. Tata, Probing charginos and neutralinos beyond the reach of LEP at the Tevatron collider. Phys. Rev. D 47, 2739 (1993)

269. G.L. Kane, C.F. Kolda, L. Roszkowski, J.D. Wells, Study of constrained minimal supersymmetry. Phys. Rev. D 49, 6173 (1994)

270. B. Abbott et al., Search for $R$-parity violating supersymmetry in the dielectron channel. Phys. Rev. Lett. 83, 4476 (1999)

271. A. Aktas et al., Search for bosonic stop decays in $R$-parity violating supersymmetry in $e^{+} p$ collisions at HERA. Phys. Lett. B 599, 159 (2004)

272. T. Kon, T. Matsushita, T. Kobayashi, Possible excess in charged current events with high $Q^{2}$ at HERA from stop and sbottom production. Mod. Phys. Lett. A 12, 3143 (1997)

273. G.F. Giudice, R. Rattazzi, Theories with gauge mediated supersymmetry breaking. Phys. Rep. 322, 419 (1999)

274. A. Aktas et al., Search for light gravitinos in events with photons and missing transverse momentum at HERA. Phys. Lett. B 616, $31(2005)$

275. S. Dimopoulos, M. Dine, S. Raby, S.D. Thomas, Experimental signatures of low-energy gauge mediated supersymmetry breaking. Phys. Rev. Lett. 76, 3494 (1996)

276. P.A.M. Dirac, Quantized singularities in the electromagnetic field. Proc. R. Soc. Lond. A 133, 60 (1931)

277. R.D. Sorkin, Kaluza-Klein monopole. Phys. Rev. Lett. 51, 87 (1983)

278. D.J. Gross, M.J. Perry, Magnetic monopoles in Kaluza-Klein theories. Nucl. Phys. B 226, 29 (1983)

279. E. Bergshoeff, E. Eyras, Y. Lozano, The massive Kaluza-Klein monopole. Phys. Lett. B 430, 77 (1998)
280. A.M. Polyakov, Particle spectrum in the quantum field theory. JETP Lett. 20, 194 (1974)

281. G. 't Hooft, Magnetic monopoles in unified gauge theories. Nucl. Phys. B 79, 276 (1974)

282. H. Minakata, Magnetic monopoles in supersymmetric grand unification. Phys. Lett. B 155, 352 (1985)

283. J.L. Lopez, Supersymmetry: from the Fermi scale to the Planck scale. Rep. Prog. Phys. 59, 819 (1996)

284. T.G. Rizzo, G. Senjanovic, Can there be low intermediate mass scales in grand unified theories? Phys. Rev. Lett. 46, 1315 (1981)

285. J. Preskill, Magnetic monopoles. Ann. Rev. Nucl. Part. Sci. 34, 461 (1984)

286. E.J. Weinberg, D. London, J.L. Rosner, Magnetic monopoles with Z(n) charges. Nucl. Phys. B 236, 90 (1984)

287. T.W. Kirkman, C.K. Zachos, Asymptotic analysis of the monopole structure. Phys. Rev. D 24, 999 (1981)

288. Y.S. Yang, Dually charged particle-like solutions in the WeinbergSalam theory. Proc. R. Soc. Lond. A 454, 155 (1998)

289. Y.M. Cho, Analytic electroweak dyon (2002)

290. F.A. Bais, W. Troost, Zero modes and bound states of the supersymmetric monopole. Nucl. Phys. B 178, 125 (1981)

291. T. Banks, M. Dine, H. Dykstra, W. Fischler, Magnetic monopole solutions of string theory. Phys. Lett. B 212, 45 (1988)

292. H.V. Klapdor-Kleingrothaus, K. Zuber, Patricle Astrophysics (The Institute of Physics, UK, 1999)

293. L.W. Alvarez et al., Search for magnetic monopoles in the lunar sample. Science 167, 701 (1970)

294. A. Aktas et al., A direct search for stable magnetic monopoles produced in positron-proton collisions at HERA. Eur. Phys. J. C 41, 133 (2005)

295. L.P. Gamberg, G.R. Kalbfleisch, K.A. Milton, Difficulties with photonic searches for magnetic monopoles (1998)

296. 2G Enterprises, http://www.2genterprises.com. Accessed 8 June 2016

297. M. Ambrosio et al., Final results of magnetic monopole searches with the MACRO experiment. Eur. Phys. J. C 25, 511 (2002)

298. S. Balestra et al., Magnetic monopole search at high altitude with the SLIM experiment. Eur. Phys. J. C 55, 57 (2008)

299. D.P. Hogan, D.Z. Besson, J.P. Ralston, I. Kravchenko, D. Seckel, Relativistic magnetic monopole flux constraints from RICE. Phys. Rev. D 78, 075031 (2008)

300. M. Detrixhe et al., Ultra-relativistic magnetic monopole search with the ANITA-II balloon-borne radio interferometer. Phys. Rev. D 83, 023513 (2011)

301. R. Abbasi et al., Search for relativistic magnetic monopoles with IceCube. Phys. Rev. D 87(2), 022001 (2013)

302. M.G. Aartsen et al., Search for non-relativistic magnetic monopoles with IceCube. Eur. Phys. J. C 74(7), 2938 (2014)

303. R.R. Ross, P.H. Eberhard, L.W. Alvarez, R.D. Watt, Search for magnetic monopoles in lunar material using an electromagnetic detector. Phys. Rev. D 8, 698 (1973)

304. J.M. Kovalik, J.L. Kirschvink, New superconducting quantum interface device based constraints on the abundance of magnetic monopoles trapped in matter: An investigation of deeply buried rocks. Phys. Rev. A 33, 1183 (1986)

305. H. Jeon, M.J. Longo, Search for magnetic monopoles trapped in matter. Phys. Rev. Lett. 75, 1443 (1995) (erratum. Phys. Rev. Lett. 76, 159 (1996)

306. K. Bendtz, D. Milstead, H.-P. Hächler, A.M. Hirt, P. Mermod, P. Michael, T. Sloan, C. Tegner, S.B. Thorarinsson, Search for magnetic monopoles in polar volcanic rocks. Phys. Rev. Lett. 110(12), 121803 (2013)

307. M. Fairbairn, A.C. Kraan, D.A. Milstead, T. Sjöstrand, Peter Z. Skands, T. Sloan, Stable massive particles at colliders. Phys. Rep. 438, 1 (2007) 
308. B. Aubert, P. Musset, M. Price, J.P. Vialle, Search for magnetic monopoles in proton-antiproton interactions at $540 \mathrm{GeV} \mathrm{cm}$ energy. Phys. Lett. B 120, 465 (1983)

309. P. Musset, M. Price, E. Lohrmann, Search for magnetic monopoles in electron-positron collisions at $34 \mathrm{GeV} \mathrm{cm}$ energy. Phys. Lett. B 128, 333 (1983)

310. B. Abbott et al., A search for heavy point-like Dirac monopoles. Phys. Rev. Lett. 81, 524 (1998)

311. A. Abulencia et al., Direct search for Dirac magnetic monopoles in $p \bar{p}$ collisions at $\sqrt{s}=1.96 \mathrm{TeV}$. Phys. Rev. Lett. 96, 201801 (2006)

312. G. Abbiendi et al., Search for Dirac magnetic monopoles in $e^{+} e^{-}$ collisions with the OPAL detector at LEP2. Phys. Lett. B 663, 37 (2008)
313. G. Aad et al., Search for magnetic monopoles in $\sqrt{s}=7 \mathrm{TeV} p p$ collisions with the ATLAS detector. Phys. Rev. Lett. 109, 261803 (2012)

314. A. De Roeck, H.P. Hächler, A.M. Hirt, M.-D. Joergensen, A. Katre, P. Mermod, D. Milstead, T. Sloan, Development of a magnetometer-based search strategy for stopped monopoles at the Large Hadron Collider. Eur. Phys. J. C 72, 2212 (2012)

315. G. Aad et al., Search for magnetic monopoles and stable particles with high electric charges in $8 \mathrm{TeV} p p$ collisions with the ATLAS detector. Phys. Rev. D 93(5), 052009 (2016) 